W. 2860

LA-7861-MS

Informal Report

SPECTRA-IV, Benchmark Spectra Based on LIB-IV

MASTER

Bhyshom

LOS ALAMO SCIENTIFIC LABORATORY

Post Office Box 1663 Los Alamios. New Mexico 87545 
LA-7861-MS

Informal Report

UC-79d

Base Technology

Issued: June 1979

\title{
SPECTRA-IV, Benchmark Spectra Based on LIB-iV
}

\author{
R. B. Kidman
}

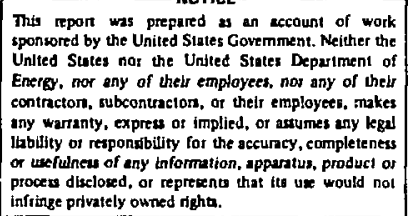

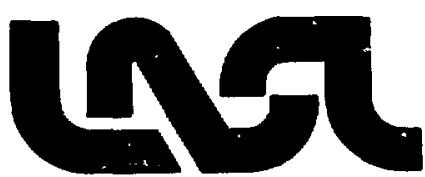


SPECTRA-IV, BENCHMARK SPECTRA BASED ON LIB-IV

by

R. B. Kidman

\begin{abstract}
Flux and adjoint spectra for each of the Cross Section Evaluation Working Group fast reactor benchmarks are numerically and graphically presented in this handbook. The spectra were computed using a 50-group crosssection library (LIB-IV) that was generated with the MINX processing code using ENDF/B-IV data. The numerical tables show the computed group numbers and allow one to observe the effects of $1-D$ and $2-D$ diffusion theory and $S_{16} P_{1 / 2}$ transport theory on the spectra. The graphical figures compare smooth interpolated curves for the central flux, edge flux, central adjoint, transport reaction rate, and original cross-section averaging weighting function.
\end{abstract}

\title{
I. INTRODUCTION
}

The Cross Section Evaluation Working Group (CSEWG) fast reactor benchmarks ${ }^{I}$ are extensively used by various laboratories for methods and data testing. The latest most comprehensive use of these 17 benchmarks at the Los Alamos Scientific Laboratory (LASL) has been documented in Ref. 2.

The flux and adjoint spectra from those calculations are numerically and graphically presented in this handbook. This handbook represents a further investigation of our data and methods and provides a convenient opportunity to collectively consider and study the spectra of all the benchmarks.

\section{CODES AND DATA}

A brief outline of the calculational procedure will provide adequate background for understanding the spectra. 
The computer code $\mathrm{MINX}^{3}$ was used to generate a 50-group library (LIB-IV ${ }^{4}$ ) from the ENDF/B-IV ${ }^{5}$ data. IIB-IV and the benchmark specifications ${ }^{1}$ were used in the computer code $1 \mathrm{DX}^{6}$ to compute one-dimensional (1-D) diffusion theory flux and adjoint spectra. The effective cross sections generated with 1DX were also used in the computer code $2 \mathrm{DB}^{7}$ to compute two-dimensional (2-D) diffusion theory flux and adjoint spectra. 1DX was also used to generate effective cross sections for the computer code ONETRAN. ${ }^{8}$ ONETRAN was then used to compute $1-\mathrm{D}, \mathrm{S}_{16}{ }^{\mathrm{P}}{ }_{1 / 2}$ transport theory flux and adjoint spectra.

The 50-group structure is shown in every table of this report. All other calculational details and data, including the fission sourres utilized, are described in Ref. 2 .

\section{GRAPHICAL RESULTS}

Most of the figures and tables in this report are organized into appendixes to avoid long interruptions of the short text.

Appendix A contains two figures (at different scales) for each benchmark. Five curves are displayed in each figure: the central flux, edge flux, transport reaction rate, central adjoint, and LIB-IV weighting function. These curves are generated in the following manner.

Beginning with a 50-group spectrum (50 numbers) calculated with 1DX, the spectrum is first normalized so that the group sum is equal to 100 . Each group flux is then converted to flux per unit lethargy by dividing by the group lethargy width. If $\phi_{i, 0}$ is the original flux in group $i$ and $\Delta_{i}$ is the lethargy width of group $i$, this renormalizing can be written as

$$
\phi_{i}=\frac{100}{\Delta_{u_{i}}} \frac{\phi_{i, 0}}{\sum_{j=1}^{50} \phi_{j, 0}} .
$$

Each group flux per unit lethargy $\phi_{i}$ is associated with the mid-lethargy energy of the group, and an interpolation formula is used to draw a smooth curve through these energy-flux points. This procedure is used for the central. flux, edge flux, and transport reaction rate.

When such curves are compared, they reveal trends and differences much more easily than if histograms were plotted on top of each other. However, if you 
desire histograms or have doubts about the interpolation, you have access to the raw data in Appendix $B$.

The adjoint spectra do not lend themselves to simple interpolations so they are plotted as histograms. This has the benefit of showing the group structure on the figures. The adjoint spectra are normalized differently than above. First of all, the group order of the adjoint from $1 D X$ is inverted to obtain the proper correspondence between adjoint and energy. Then, if $\phi_{i, 0}^{+}$is the original adjoint (after it has been inverted) in group $i$, and $x_{i}$ is the fission source in group $i$, the normalized adjoint $\phi_{1}^{+}$is given by

$$
\phi_{i}^{+}=\frac{\phi_{i, 0}^{+}}{\sum_{j=1}^{50} x_{j} \phi_{j, 0}^{+}} .
$$

The adjoint spectra are plotted according to an expanded linear ordinate scale placed on the right side of the figures. This allows the adjoint variations to be displayed more clearly.

The LIB-IV weighting-function curve is normalized so that integrating it over the group-structure energy range will give 100. This curve is provided so that you can compare the cross-section averaging function with the spectra produced by the averaged cross sections. As an aid to judging potential trouble areas, I call your attention to the slope differences between 0.8 and $2 \mathrm{MeV}$. Similar differences have been shown ${ }^{9}$ to cause $25 \%$ errors in the fertinent group absorption cross sections for $\mathrm{Fe}$ and ${ }^{10} \mathrm{~B}$. Smaller (larger) errors occur with flatter (steeper) cross-section behavior.

Such difficulties with the LIB-IV weighting function has led to the adoption of a new weighting function (PRE-V) for the processing of ENDF/B-V data. The LIB-IV and PRE-V weighting functions are compared in Figs. 1 and 2 . The Pre-V weighting function does not have the discontinuous slope changes of the LIB-IV weighting function, and a fusion peak has been added to the PRE-V weighting function.

The normalized edge flux is included in the figures to show how the spectral shape changes as one proceeds from the core center to the reactor edge. Compared to the central flux, the edge flux has more structure because it has been influenced by more materials. 
The computer code $\mathrm{MINX}^{3}$ was used to generate a 50-group library (LIB-IV ${ }^{4}$ ) from the ENDF/B-IV ${ }^{5}$ data. LIB-IV and the benchmark specifications ${ }^{1}$ were used in the computer code $1 \mathrm{DX}^{6}$ to compute one-dimensional (1-D) diffusion theory flux and adjoint spectra. The effective cross sections generated with IDX were also used In the computer code $2 \mathrm{DB}^{7}$ to compute two-dimensional (2-D) diffusion theory flux and adjoint spectra. 1DX was also used to generate effective cross sections for the computer code ONETRAN. ${ }^{8}$ ONETRAN was then used to compute $1-\mathrm{D}, \mathrm{S}_{16}{ }^{\mathrm{P}}{ }_{1 / 2}$ transport theory flux and adjoint spectra.

The 50-group structure is shown in every table of this report. All other calculational details and data, including the fission sources utilized, are described in Ref. 2.

\section{GRAPHICAL RESULTS}

Most of the figures and tables in this report are organized into appendixes to avoid long interruptions of the short text.

Appendix A contains two figures (at different scales) for each benchmark. Five curves are displayed in each figure: the central flux, edge flux, transport reaction rate, central adjoint, and LIB-IV weighting function. These curves are generated in the following manner.

Beginning with a 50-group spectrum (50 numbers) calculated with 1DX, the spectrum is first normalized so that the group sum is equal to 100 . Each group flux is then converted to flux per unit lethargy by dividing by the group lethargy width. If $\phi_{i, 0}$ is the original flux in group $i$ and $\Delta u_{i}$ is the lethargy width of group $i$, this renormalizing can be written as

$$
\phi_{i}=\frac{100}{\Delta_{u_{i}}} \frac{\phi_{i, 0}}{\sum_{j=1}^{50} \phi_{j, 0}} .
$$

Each group flux per unit lethargy $\phi_{i}$ is associated with the mid-lethargy energy of the group, and an interpolation formula is used to draw a smooth curve through these energy-flux points. This procedure is used for the central flux, edge flux, and transport reaction rate.

When such curves are compared, they reveal trends and differences much more easily than if histograms were plctted on top of each other. However, if you 
desire histograms or have doubts about the interpolation, you have access to the raw data in Appendix $B$.

The adjoint spectra do not lend themselves to simple interpolations so they are plotted as histograms. This has the benefit of showing the group structure on the ilgures. The adjoint spectra are normalized differently than above. First of all, the group order of the adjoint from IDX is inverted to obtain the proper correspondence between adjoint and energy. Then, if $\phi_{i, 0}^{+}$is the original adjoint (after it has been inverted) in group $i$, and $\chi_{i}$ is the fission source in group 1 , the normalized adjoint $\phi_{1}^{+}$is given by

$$
\phi_{i}^{+}=\frac{\phi_{i, 0}^{+}}{\sum_{j=1}^{50} x_{j} \phi_{j, 0}^{+}} .
$$

The adjoint spectra are plotted according to an expanded linear ordinate scale placed on the right side of the figures. This allows the adjoint variations to be displayed more clearly.

The LIB-IV weighting-function curve is normalized so that integrating it over the group-structure energy range will give 100. This curve is provided so that you can compare the cross-section averaging function with the spectra produced by the averaged cross sections. As an aid to judging potential trouble areas, I call your attention to the slope differences between 0.8 and $2 \mathrm{MeV}$. Similar differences have been shown ${ }^{9}$ to cause $25 \%$ errors in the pertinent group absorption cross sections for $\mathrm{Fe}$ and ${ }^{10} \mathrm{~B}$. Smaller (larger) errors occur with flatter (steeper) cross-section behavior.

Such difficulties with the LIB-IV weighting function has led to the adoption of a new weighting function $(P R E-V)$ for the processing of FNDF/B-V data. The LIB-IV and PRE-V weighting functions are compared in Figs. 1 and 2. The Pre-V weighting function does not have the discontinuous slope changes of the LIB-IV weighting function, and a fusion peak has been added to the PRE-V weighting function.

The noralized edge flux is included in the figures to show how the spectral shape changes as one proceeds from the core center to the reactor edge. Compared to the central flux, the edge flux has more structure because it has been influenced by more materials. 


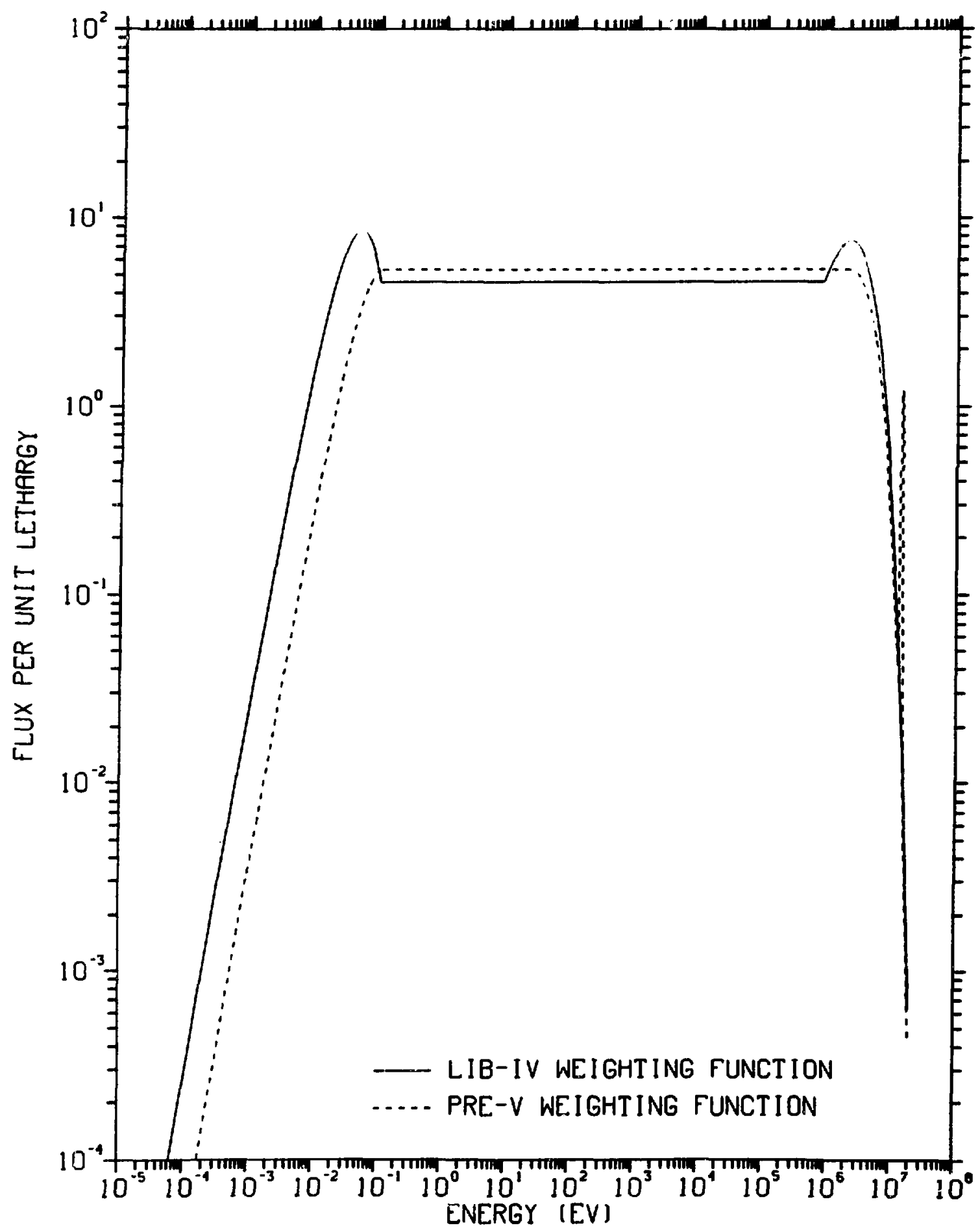

Fig. 1. Weighting functions. 


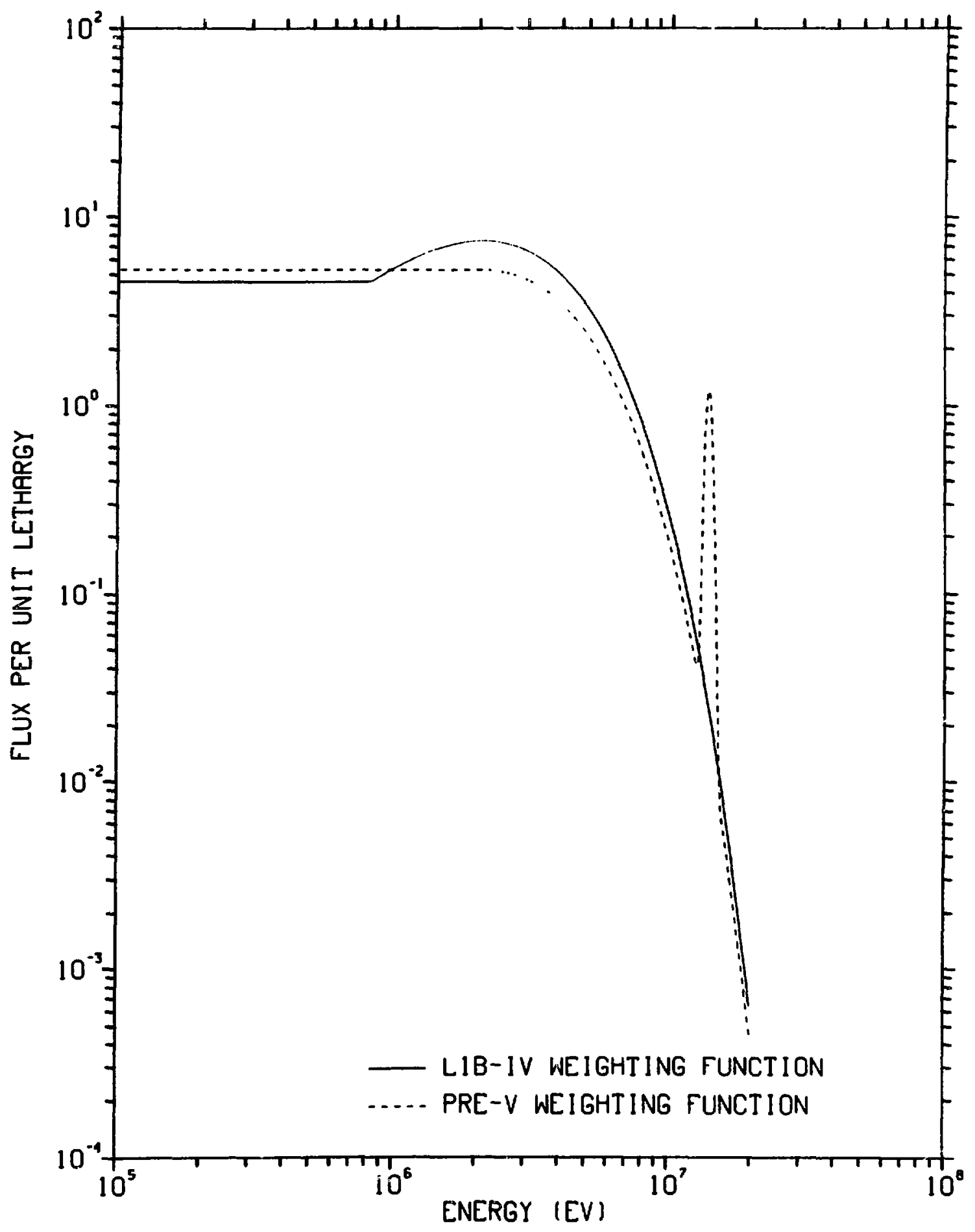

Fig. 2. Weighting functions. 
In the formulation of the shielding-factor method, the cross-section averaging weighting function is actually an approximation to the collision density, so it is somewhat unfair to compare the LIB-IV weighting iunction with the flux spectra. The product of the central flux and the central macroscopic transport cross section is included in the figures to provide a more valid comparison to the LIB-IV weighting function. The figures show that the transport reaction rate is generally smoother than the flux alone. This is encouraging because the smoother reaction rate is more easily approximated or represented with a smooth weighting function.

Occasionally it is useful to see the central flux spectra from all benchmarks plotted on the same graph, as in Figs. 3 and 4. No attempt was made to label each curve. These figures may answer some questions about the collective behavior of the spectra, or certain hypotheses can be roughly tested against the collection.

IV. NUMERICAL RESULTS

Appendix $B$ contains three tables of 50-group numerical spectra for each benchmark. The first table for each benchmark contains three versions of the central flux spectrum as calculated with 1-D diffusion theory, 1-D transport theory, and 2-D diffusion theory. The second table contains the same versions of the edge-flux spectrum, and similarly, the third table contains the central adjoint spectrum. (No 2-D calculations were made for JEZEBEL or GODIVA, and no adjoint calculations were made for SEFOR.)

The number at the bottom of the "Energy Boundary" column is the lowest energy of the 50-group structure. The numbers at the bottom of the other columns are the sums of the respective columns. All of the flux spectra have been normalized to sum to 100. All of the adjoint spectra have been normalized as described in Sec. III above.

The numerical spectra are presented to provide a precise record of the points used in drawing the curves, to allow independent curve drawing, and to compare precisely with other calculations of the spectra. More importantly, you can observe how the various modes of calculation affect the spectra. 


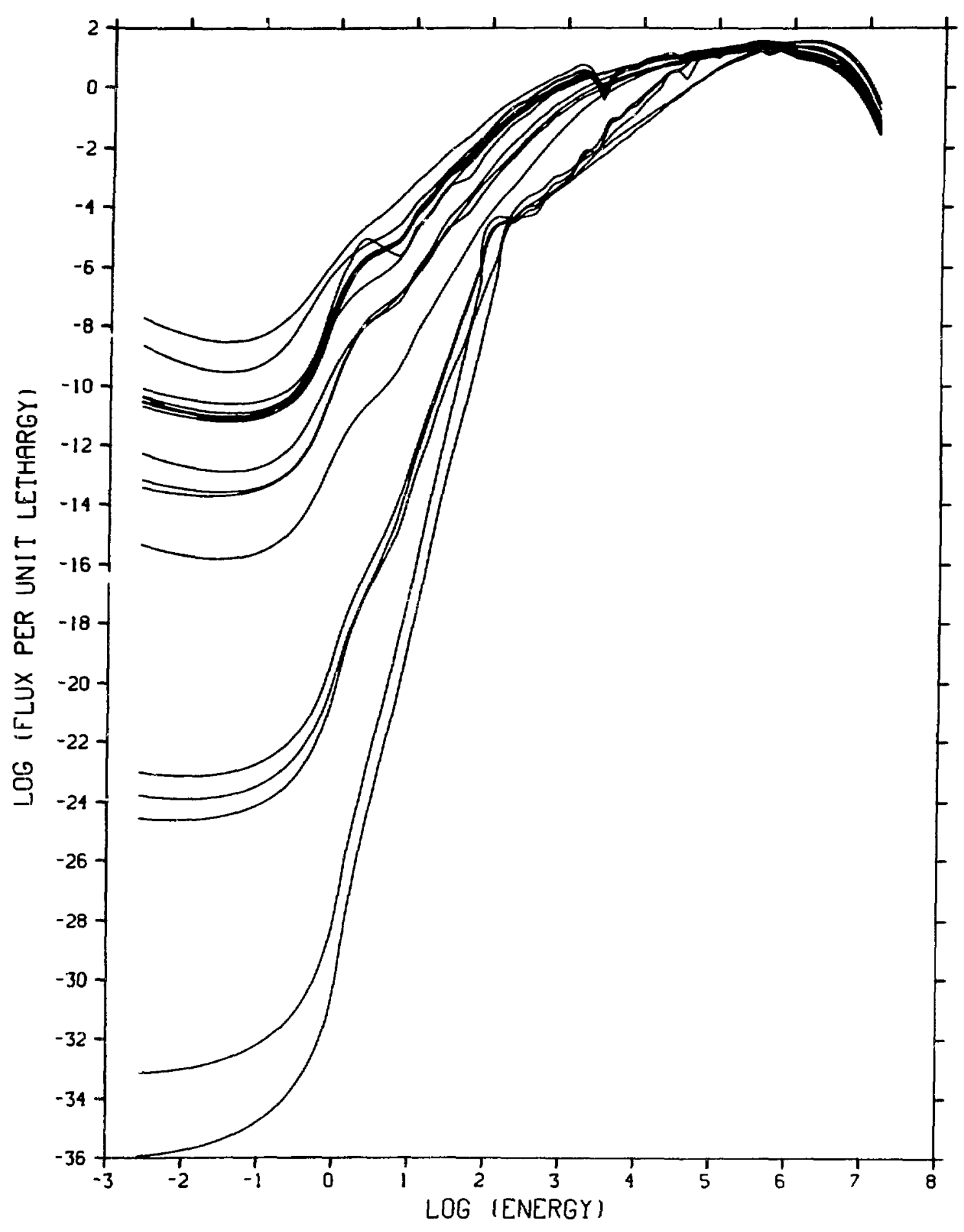

Fig. 3. Central flux spectra. 


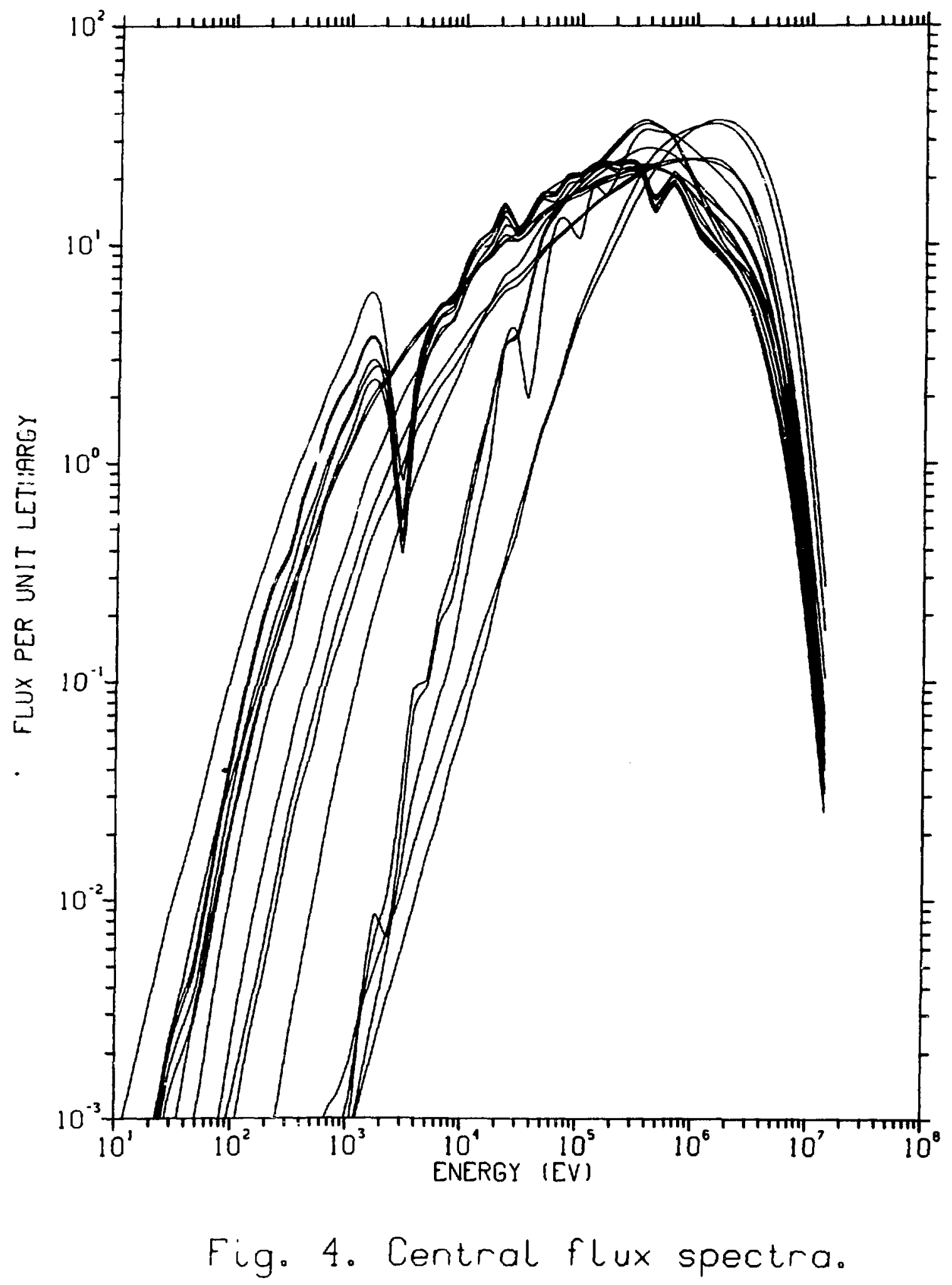




\section{REFERENCES}

1. H. Alter, R. B. Kidman, R. LaBauve, R. Protsik, and L. A. Zolator, "ENDF202 Cross Section Evaluation Working Group Benchmark Specifications," Brookhaven National Laboratory report BNL-19302 (1974).

2. R. B. Kidman, "ENDF/B-IV, LIB-IV, and the CSElvG Berichmarks," Los Alamos Scientific Laboratory report LA-7355-MS (1978).

3. C. R. Weisbin, P. D. Soran, R. E. MacFarlane, D. R. Harris, R. J. LaBauve, J. S. Hendricks, J. E. White, and R. B. Kidman, "MINX, A Multigroup Interpretation of Nuclear X-Sections from ENDF/B, "Los Alamos Scientific Laboratory report $\overline{\mathrm{L} A}-6486-\overline{\mathrm{MS}}$ (1976).

4. R. B. Kidman and R. E. MacFarlane, "LIB-IV, i Library of Group Constants for Nuclear Reactor Calculations," Los Alamos Scientific Laboratory report LA-6260-MS (1976).

5. D. Garber, Ed., "Data Formats and Procedures for the ENDF Neutron Cross Section Library," Brookhaven National Laboratory report BNL 50274 (1976).

6. R. W. Hardie and W. W. Little, Jr., "1DX, A One-Dimensional Diffusion Code for Generating Effective Nuclear Cross Sections," Battelle Northwest Laboratory report BNWL-954 (1969).

7. W. W. Little, Jr., and R. W. Hardie, "2DB User's Manual -- Revision 1," Batte1le Northwest Laboratory report BNWL-831 REV 1 (1969).

8. T. R. Hill, "ONETRAN: A Discrete Ordinates Finite Element Coce for the Solution of the One-Dimensional Multigroup Transport Equation," Los Alamos Scientific Laboratory report LA-5990-MS (1975).

9. R. B. Kidman, R. E. MacFarlane, and M. Becker, "Difterence's Between LASLand ANL-Processed Cross Sections," Los Alamos Scientific Laboratory report LA-7191-MS (1978). 


\section{APPENDIX A}

\section{GRAPHICAL DATA}

This appendix contains the spectra curves described in the text. There are two figures for each benchmark. The first figure is scaled so that the entire curve for each spectrum is contalued in the figure, i.e., an overall view. The second figure is scaled so that the more structured, more important high-energy end of each spectrum is magnified, i.e., details are more clearly revealed. There are a few places where some of the curves show unrealistic behavior. They are not caused by errors in the physics codes; they are caused by using too simple an interpolation formula in regions where the spectra change greatly. 


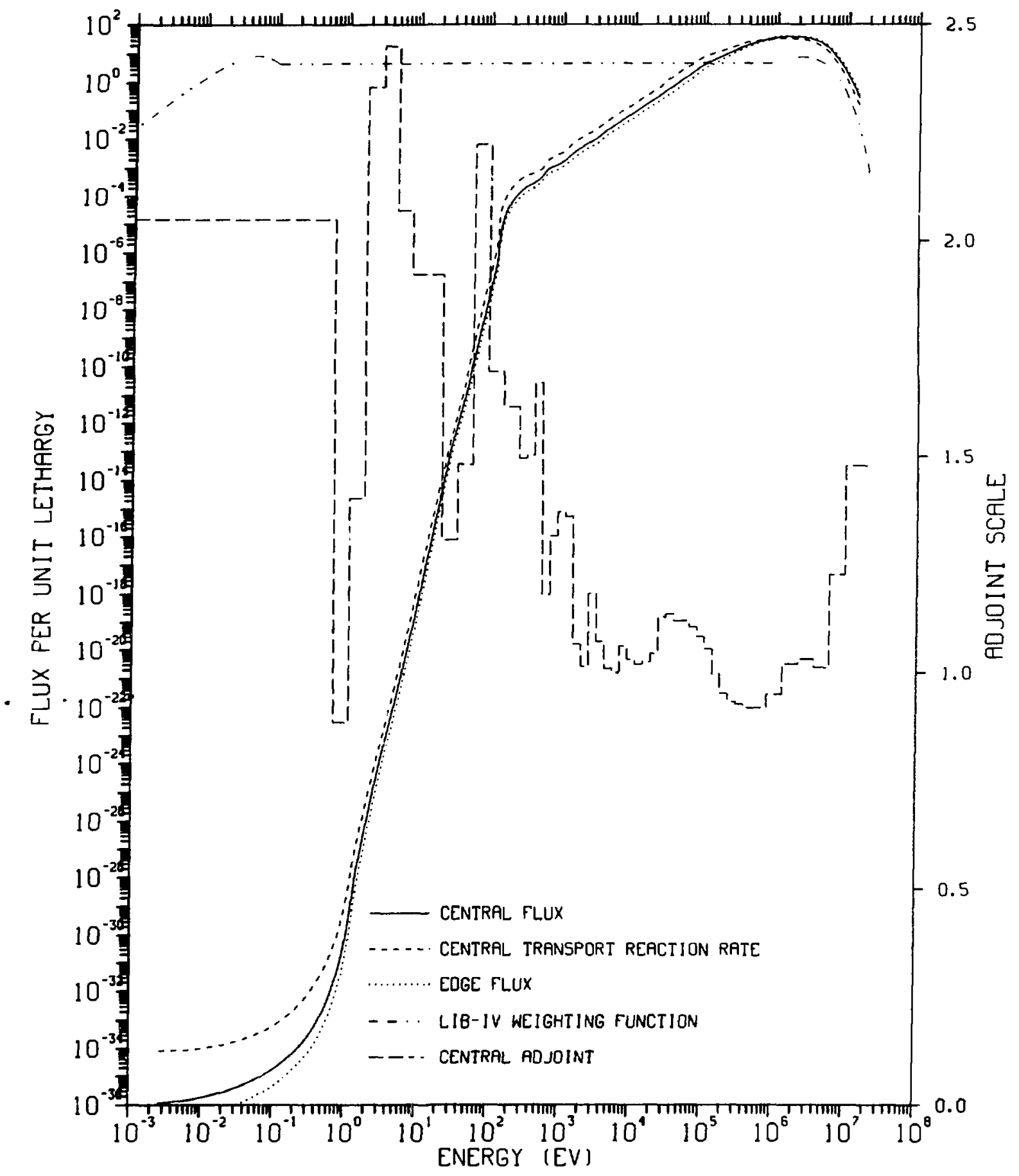

Fig. A-1. JEZEBEL spectra. 


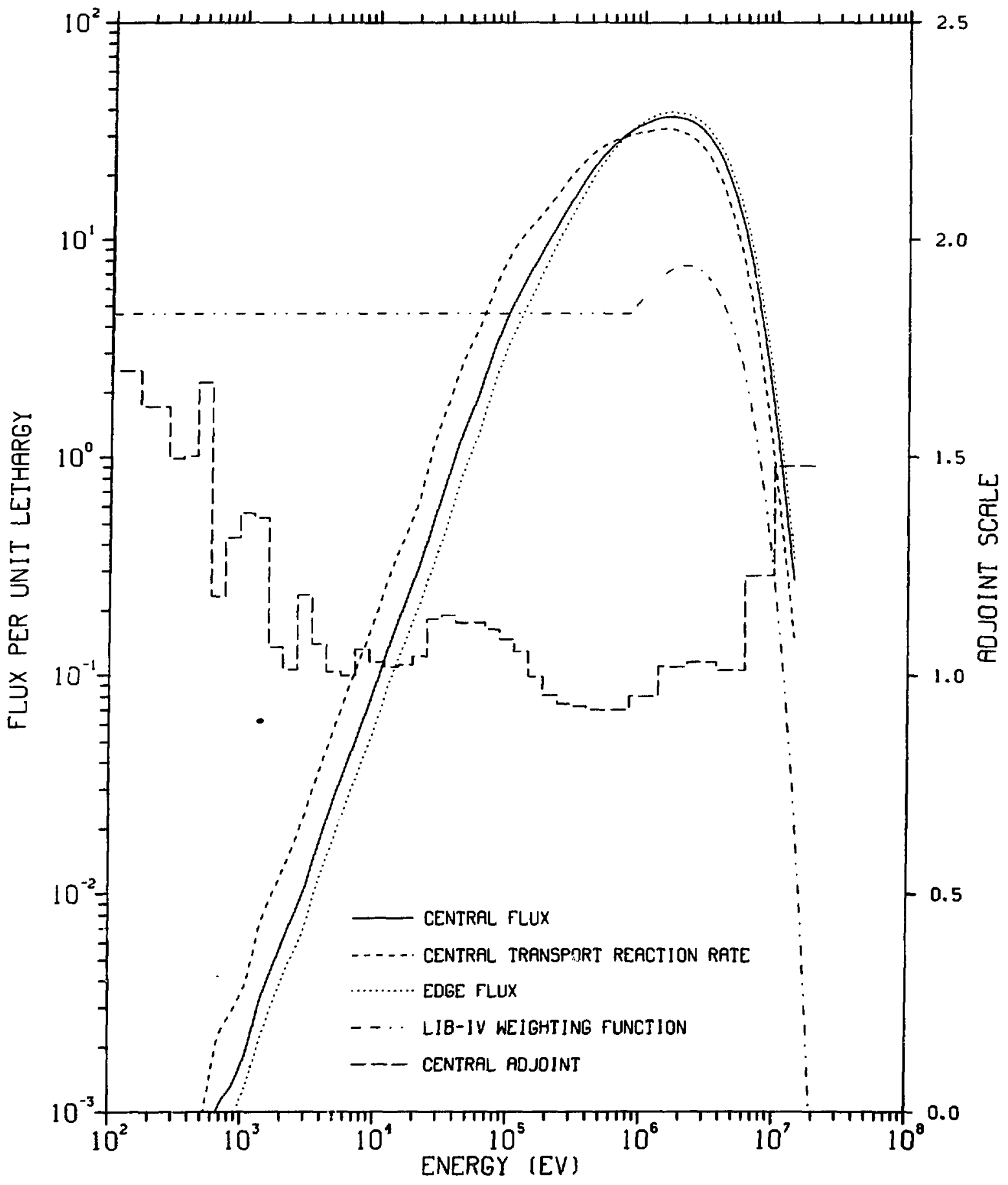

Fig. A-2. JEZEBEL spectra. 


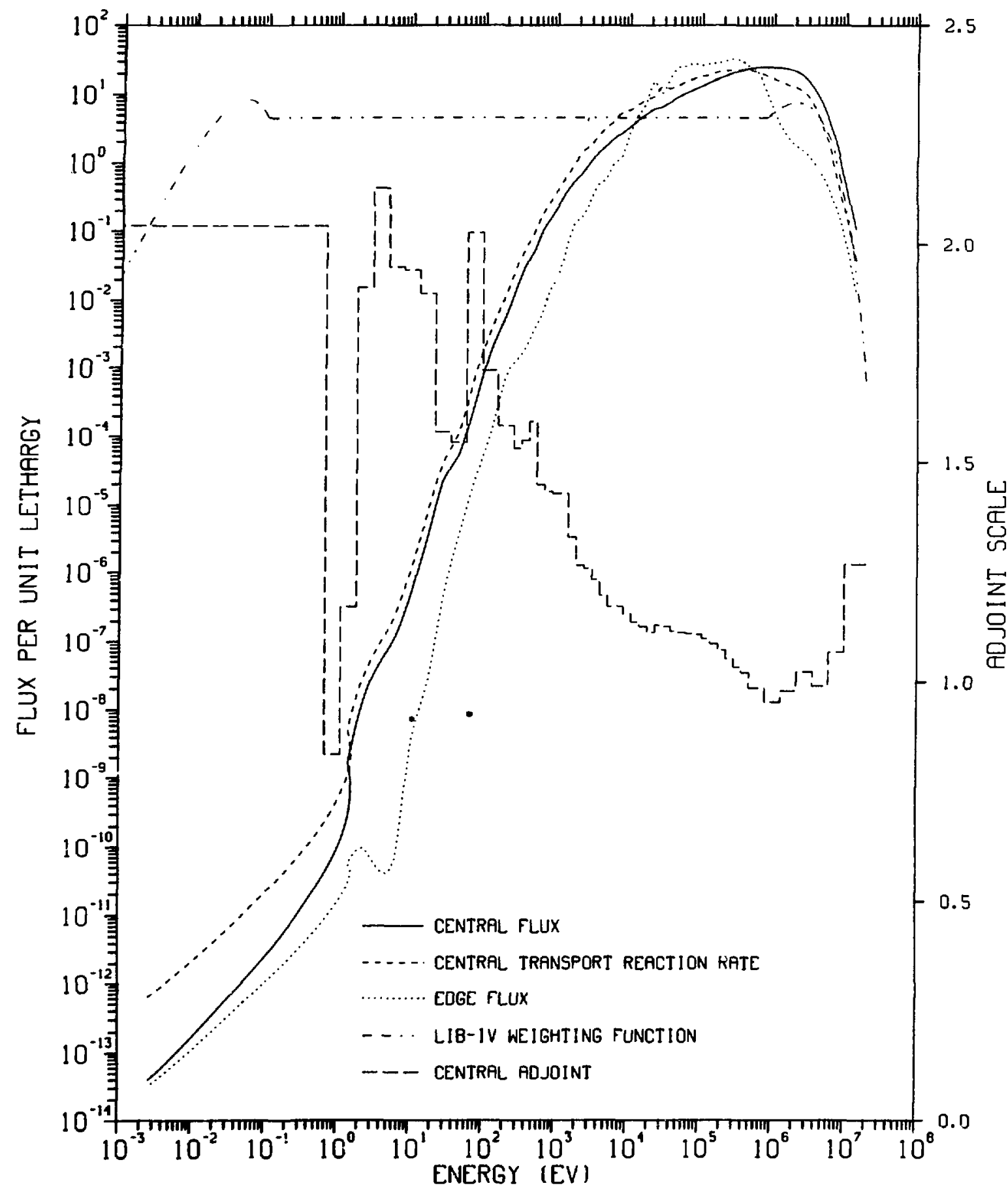

Fig. R-3. VERR-11R spectra. 


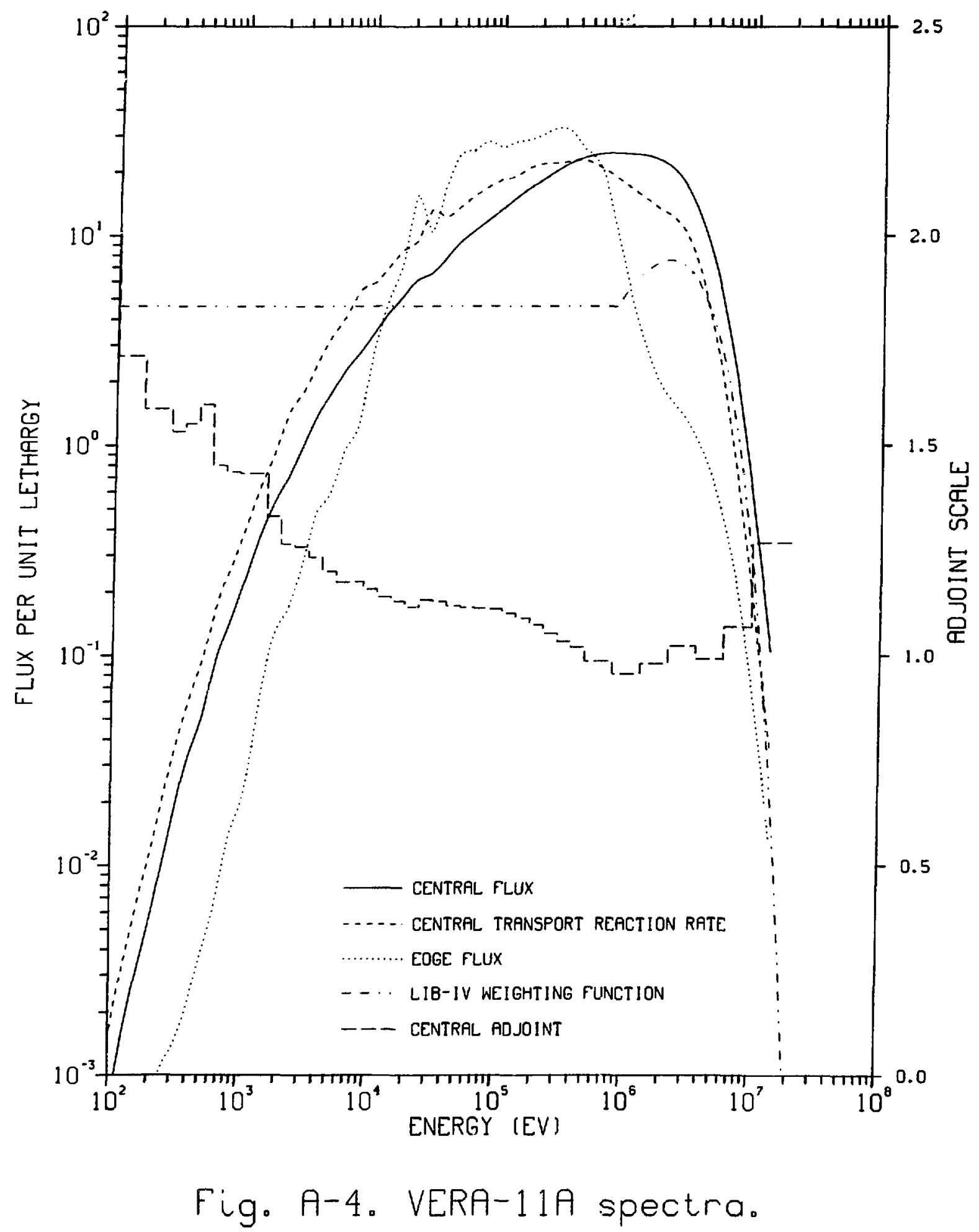




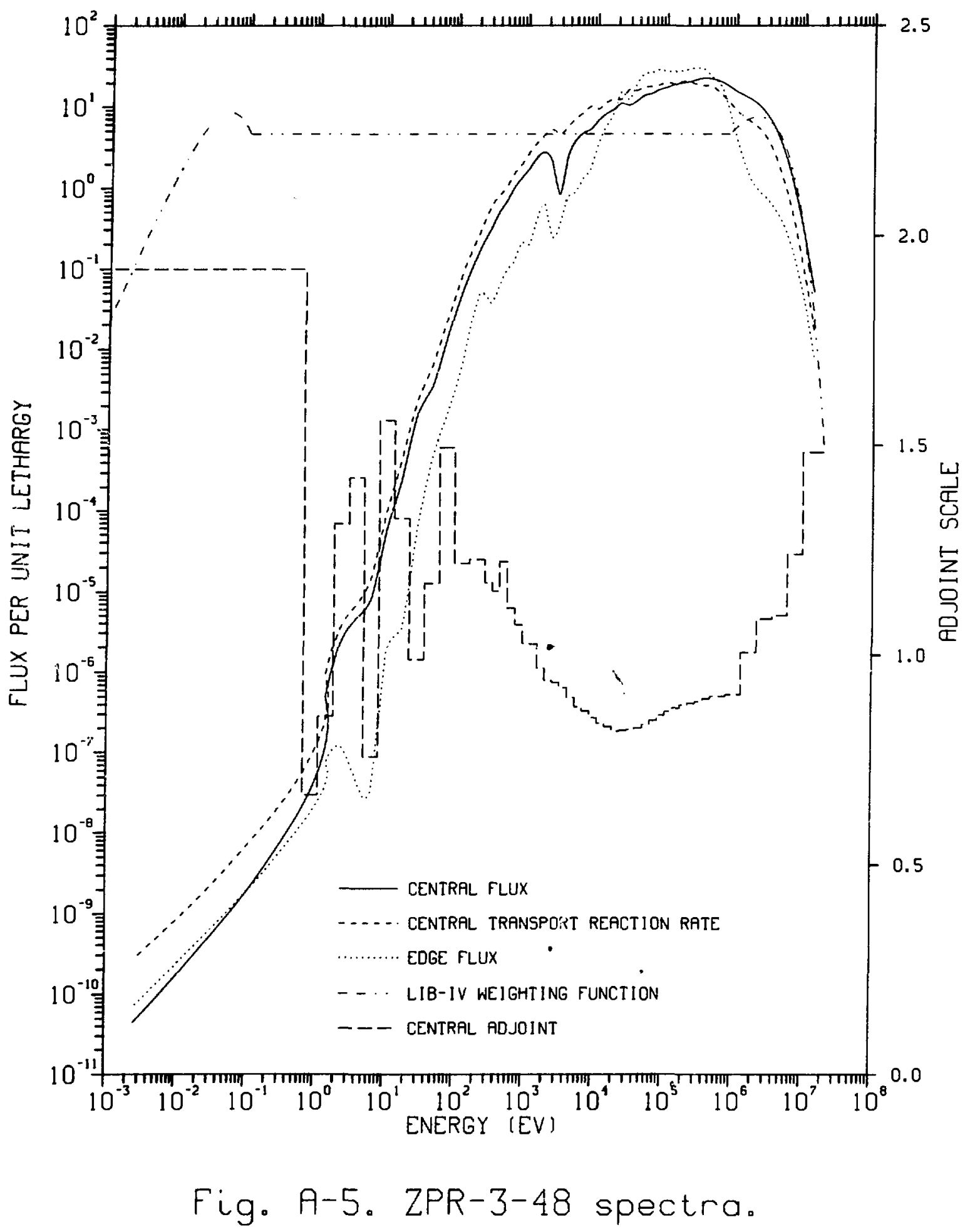




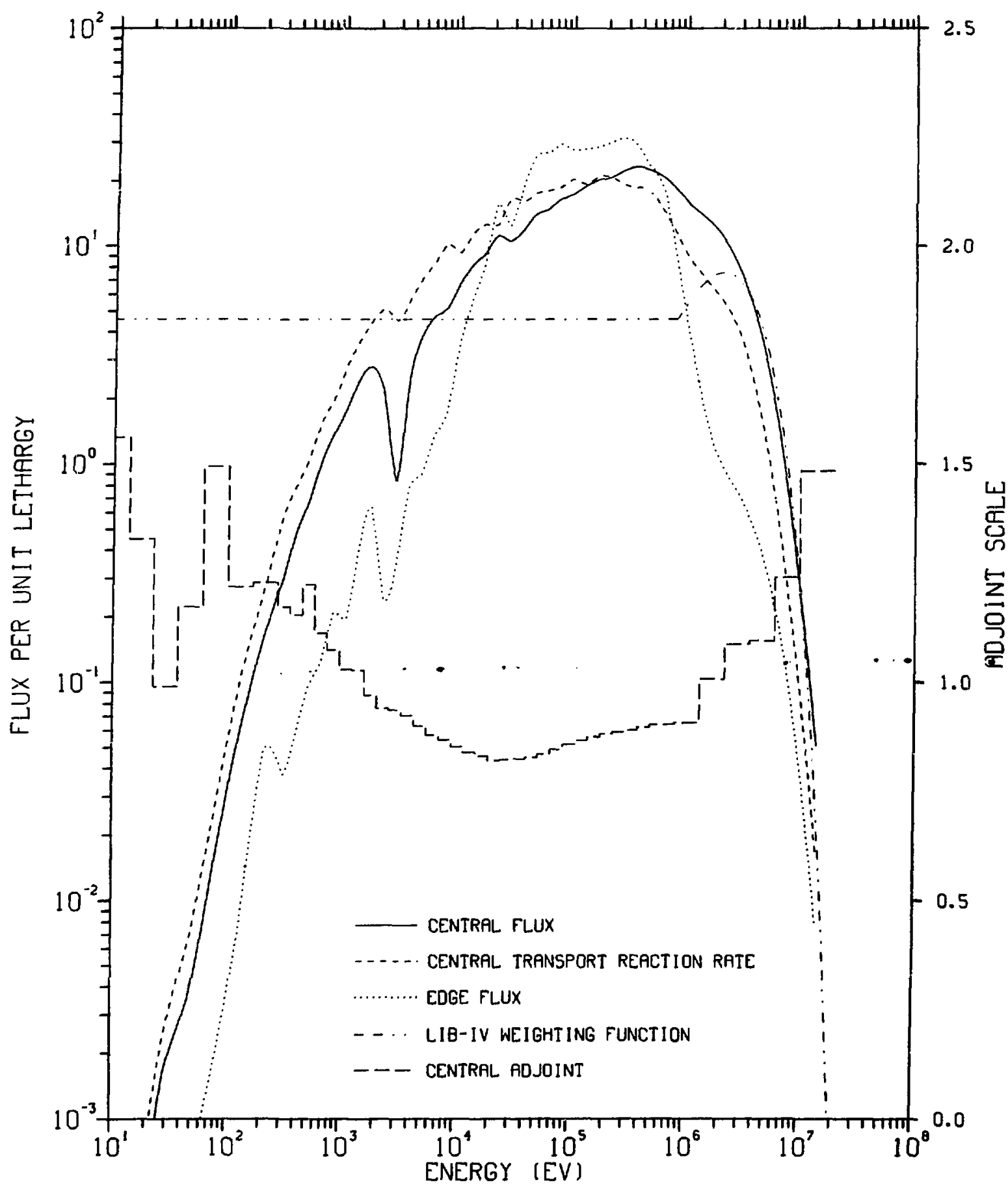

Fig. A-6. ZPR-3-48 spectra. 


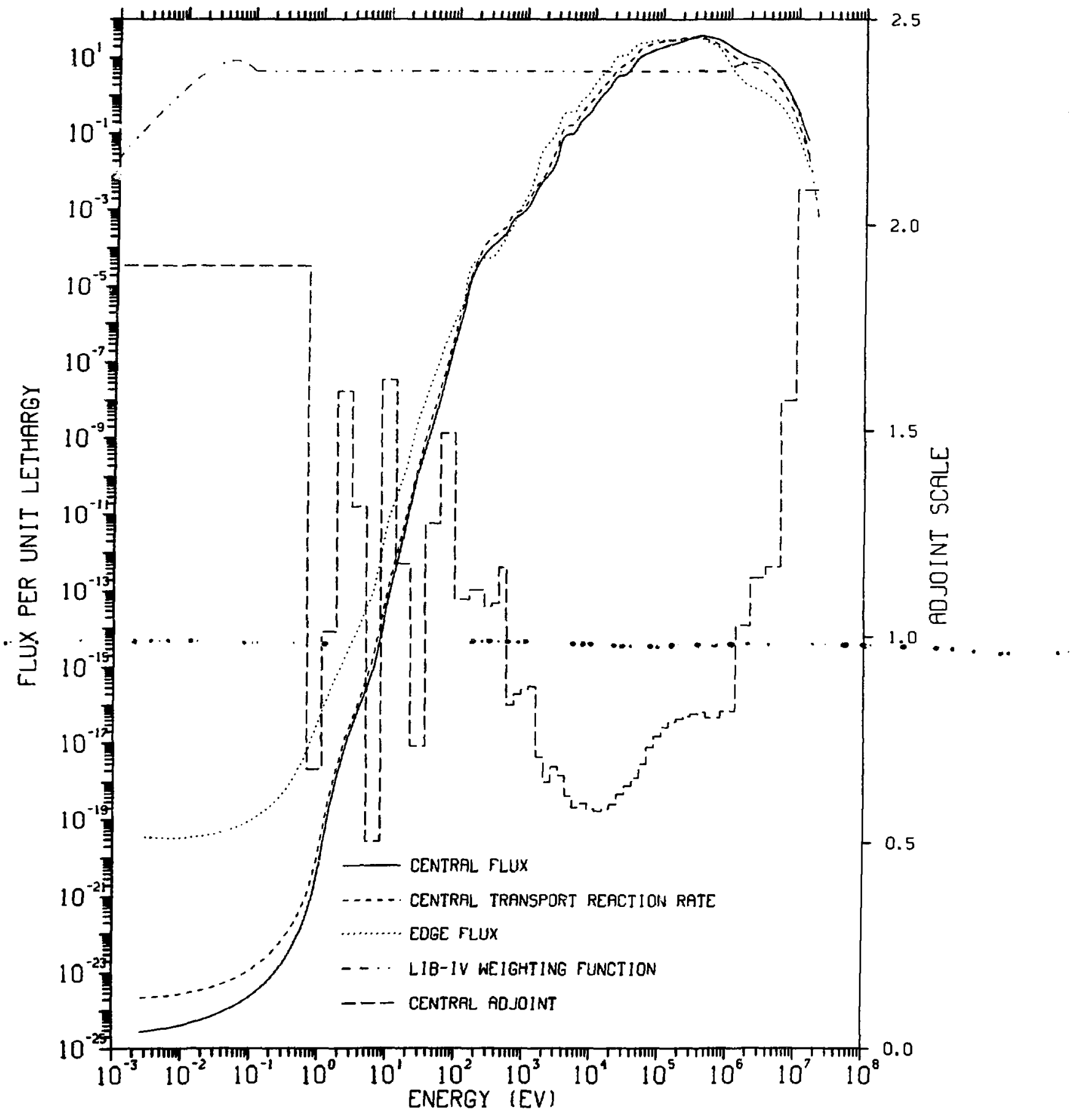

Fig. A-7. ZEBRA-3 spectra. 


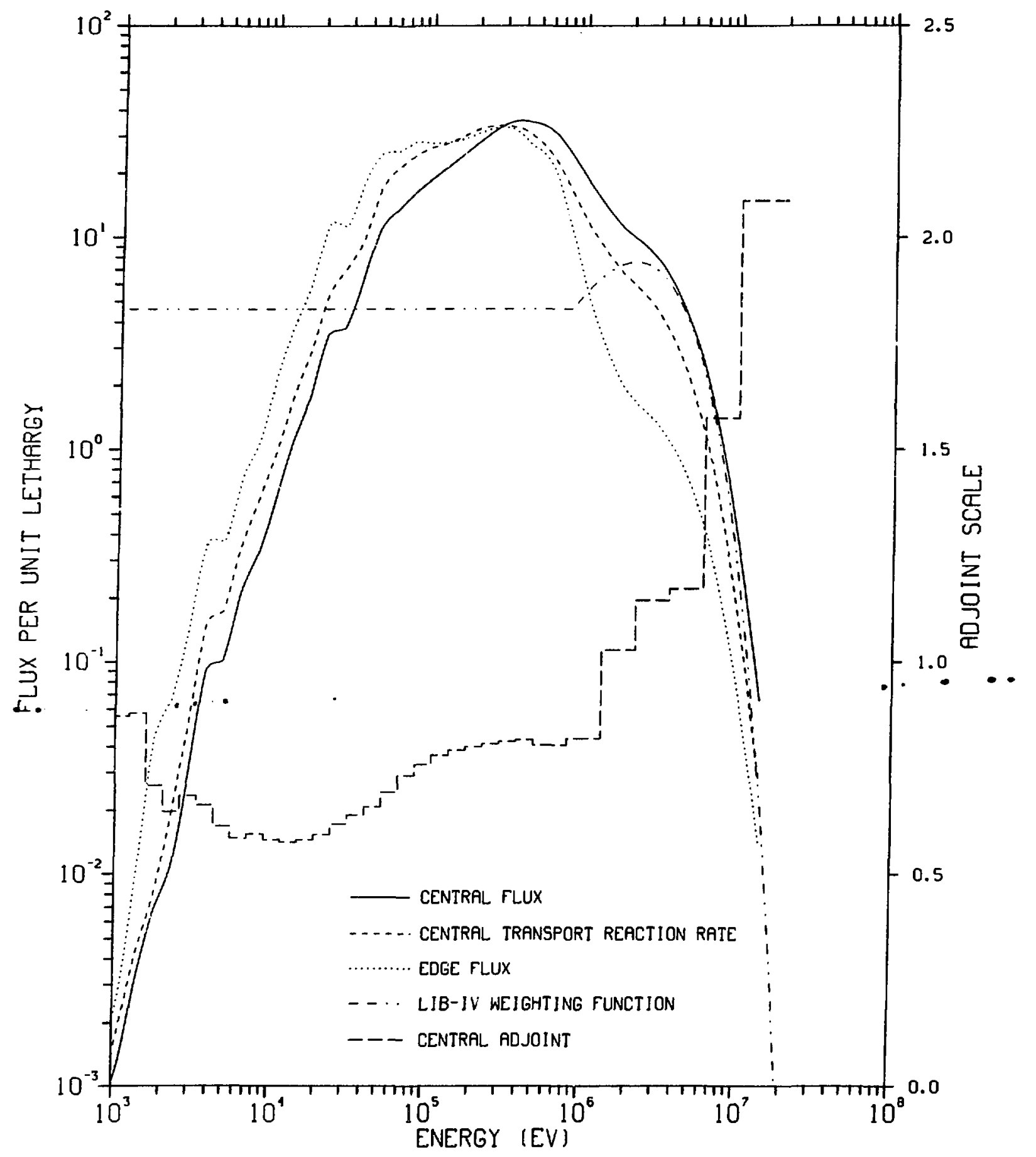

Fig. A-8. ZEBRA-3 spectra. 


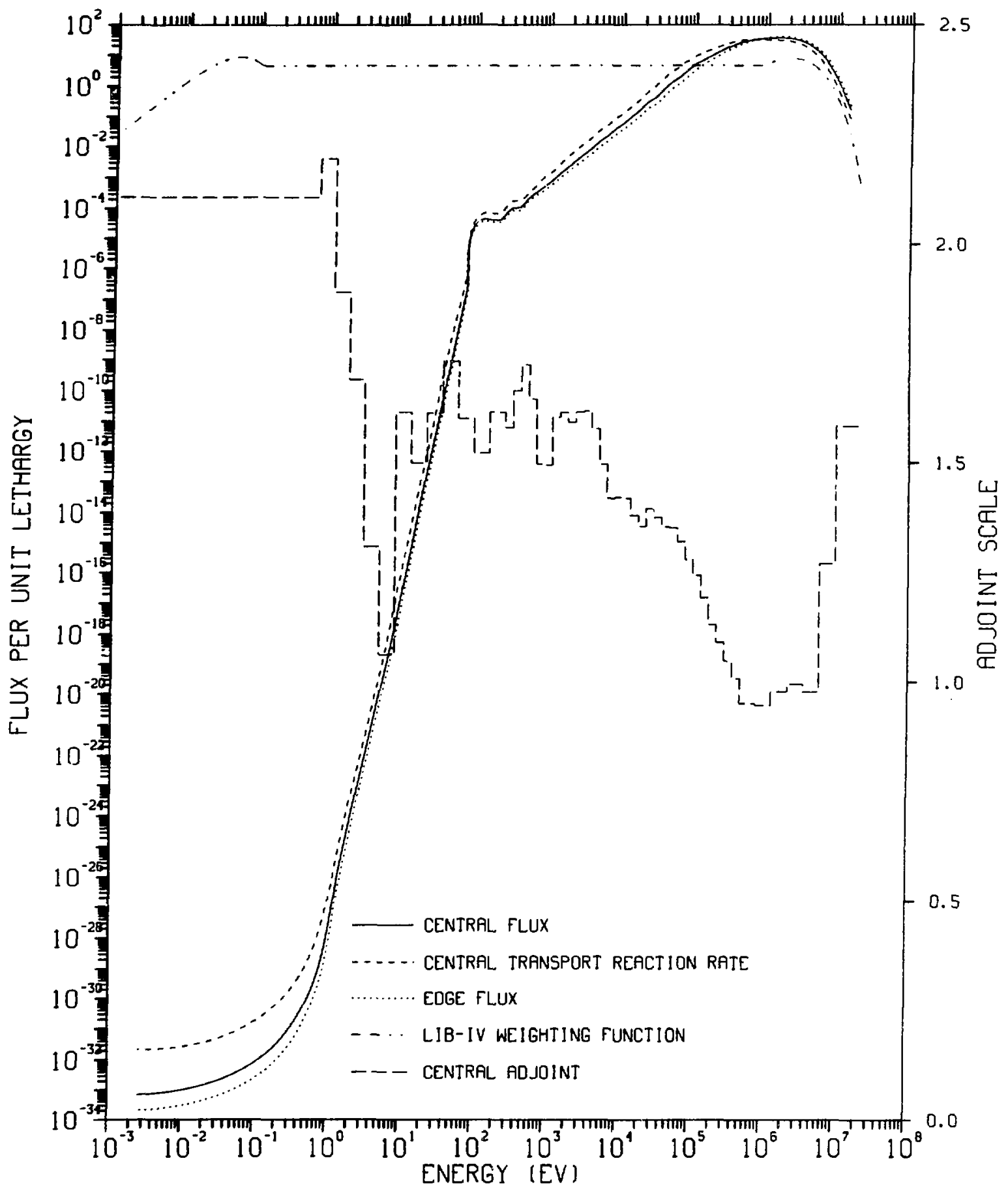

Fig. A-9. GODIVA spectra. 


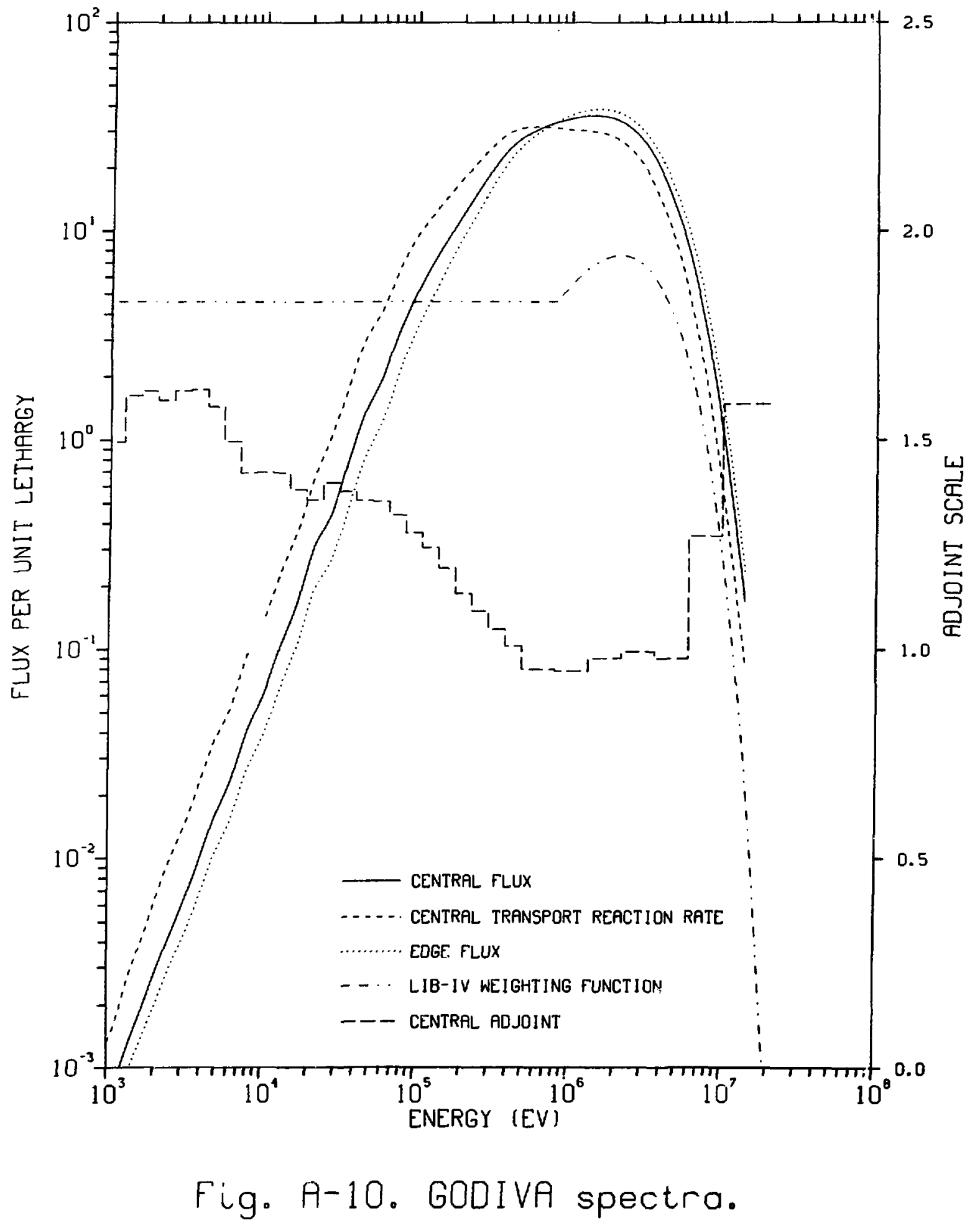




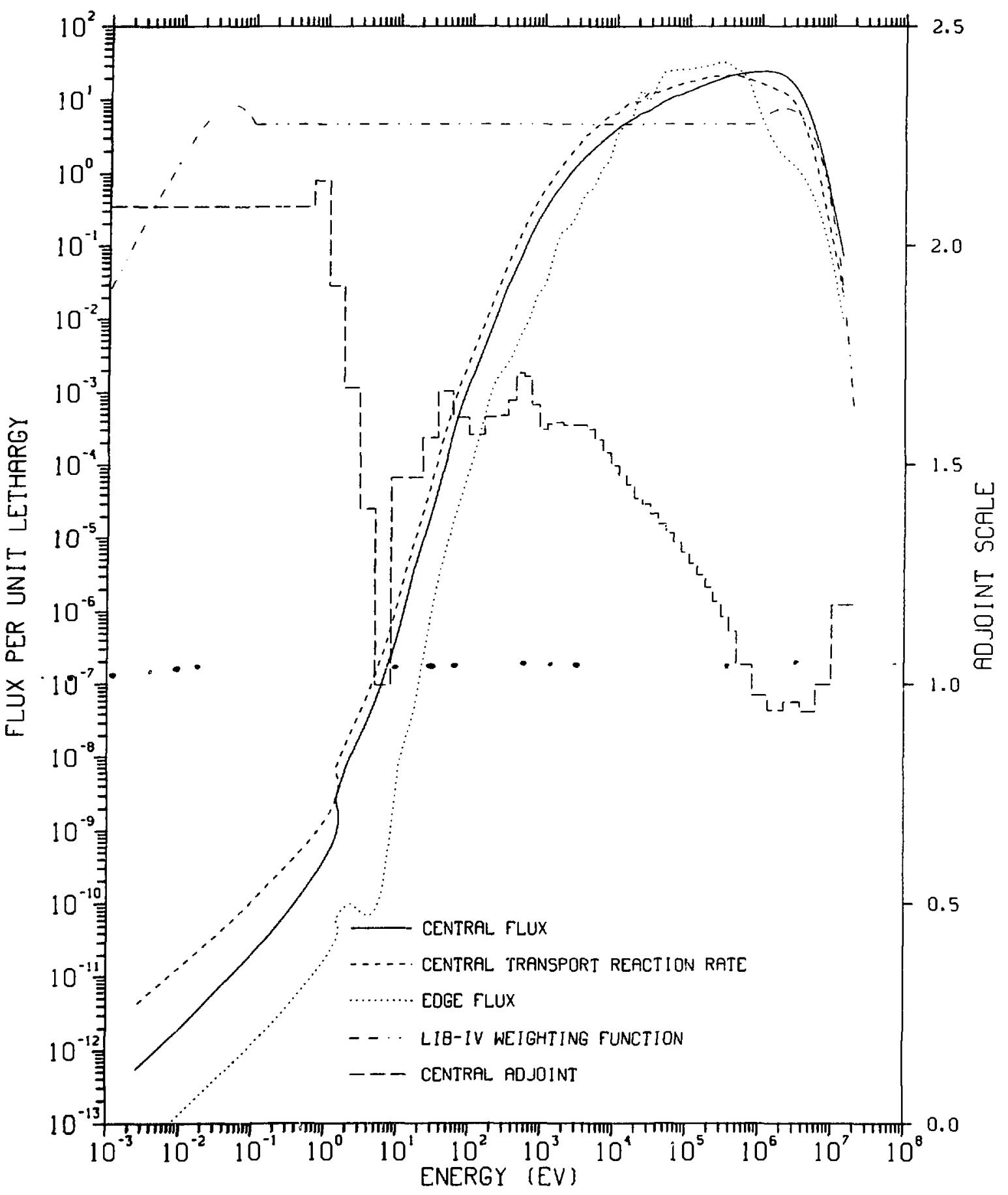

Fig. A-11. VERA-1B spectra. 


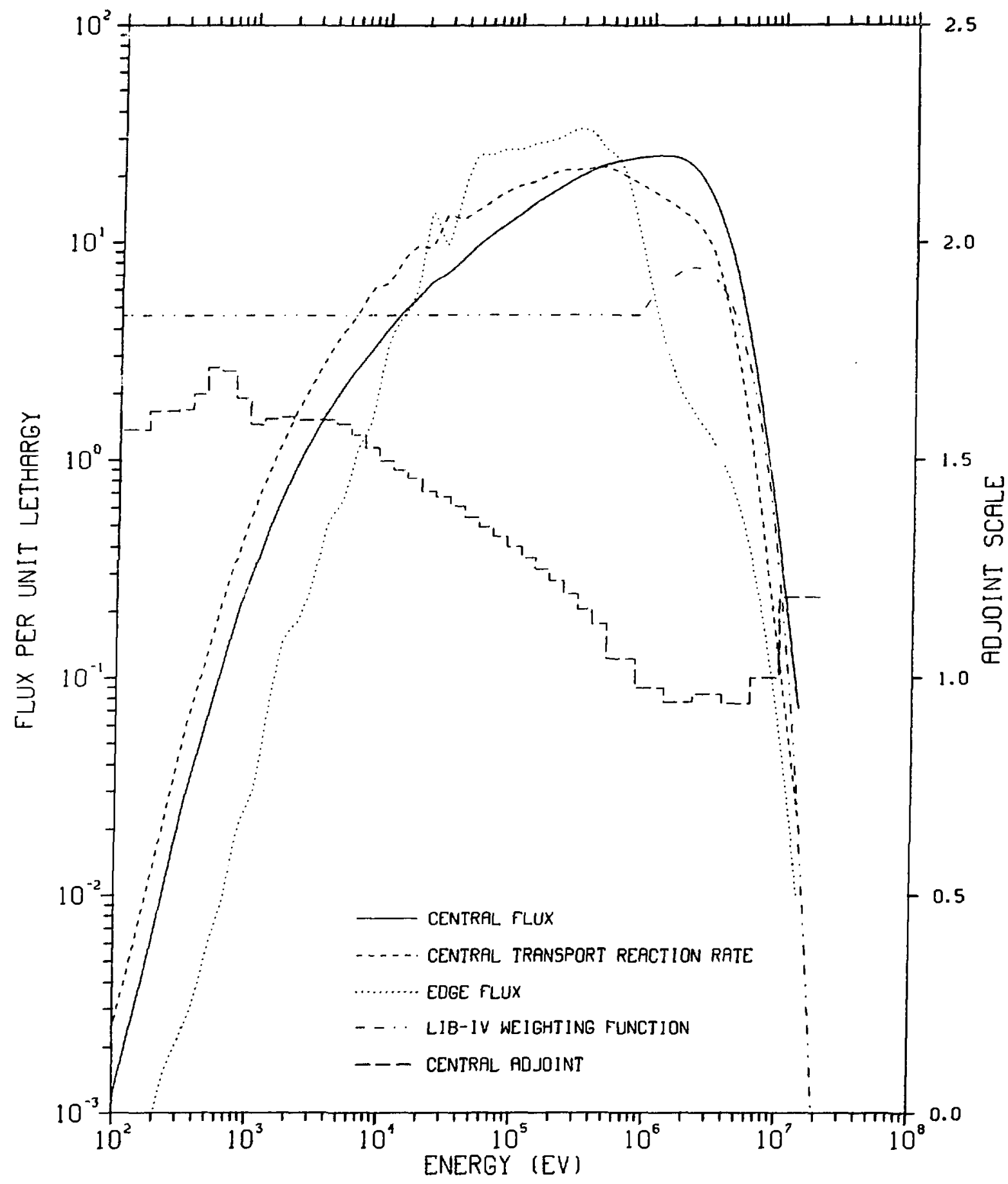

Fig. A-12. VERA-1B spectra. 


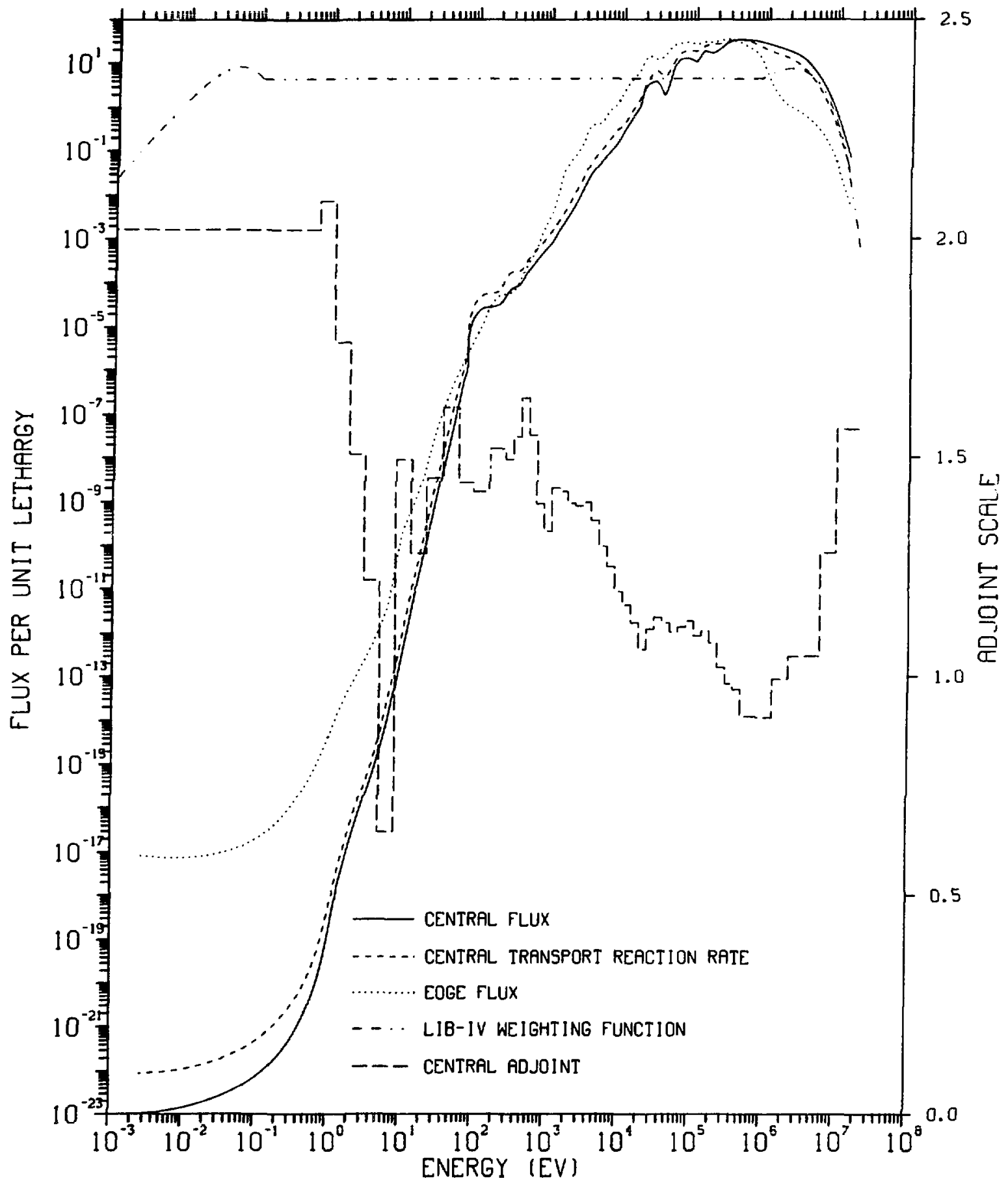

Fig. A-13. ZPR-3-6F spectra. 


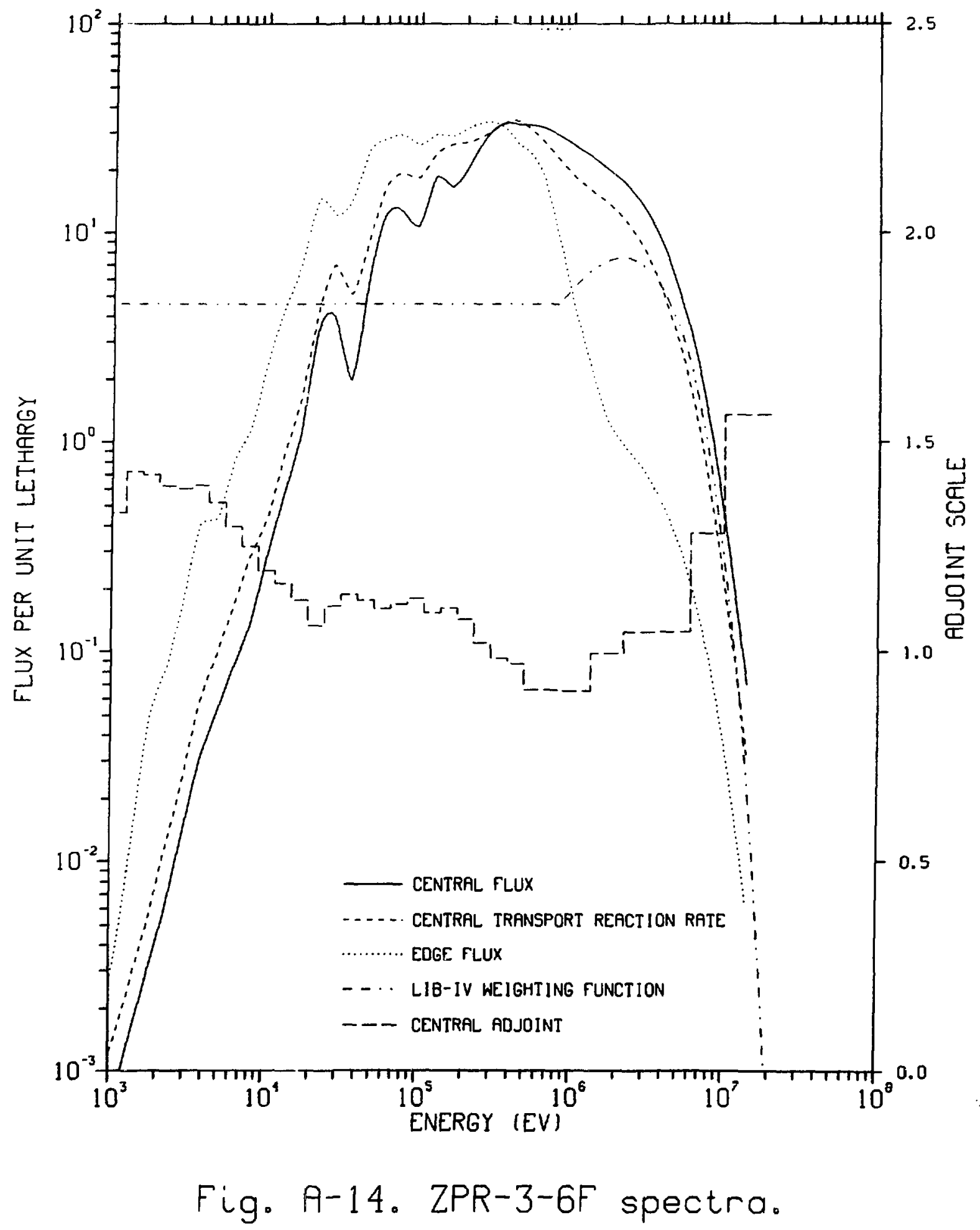




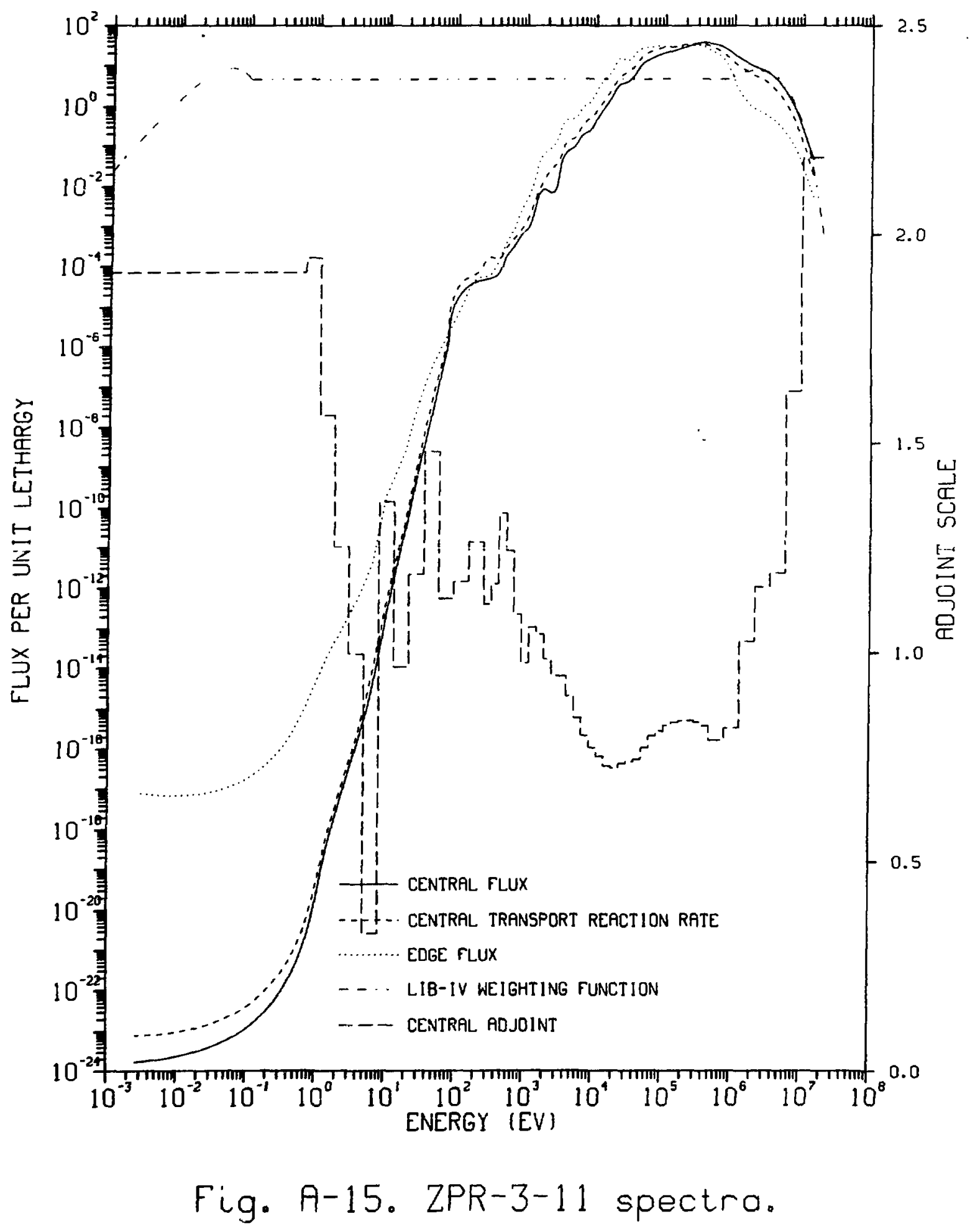




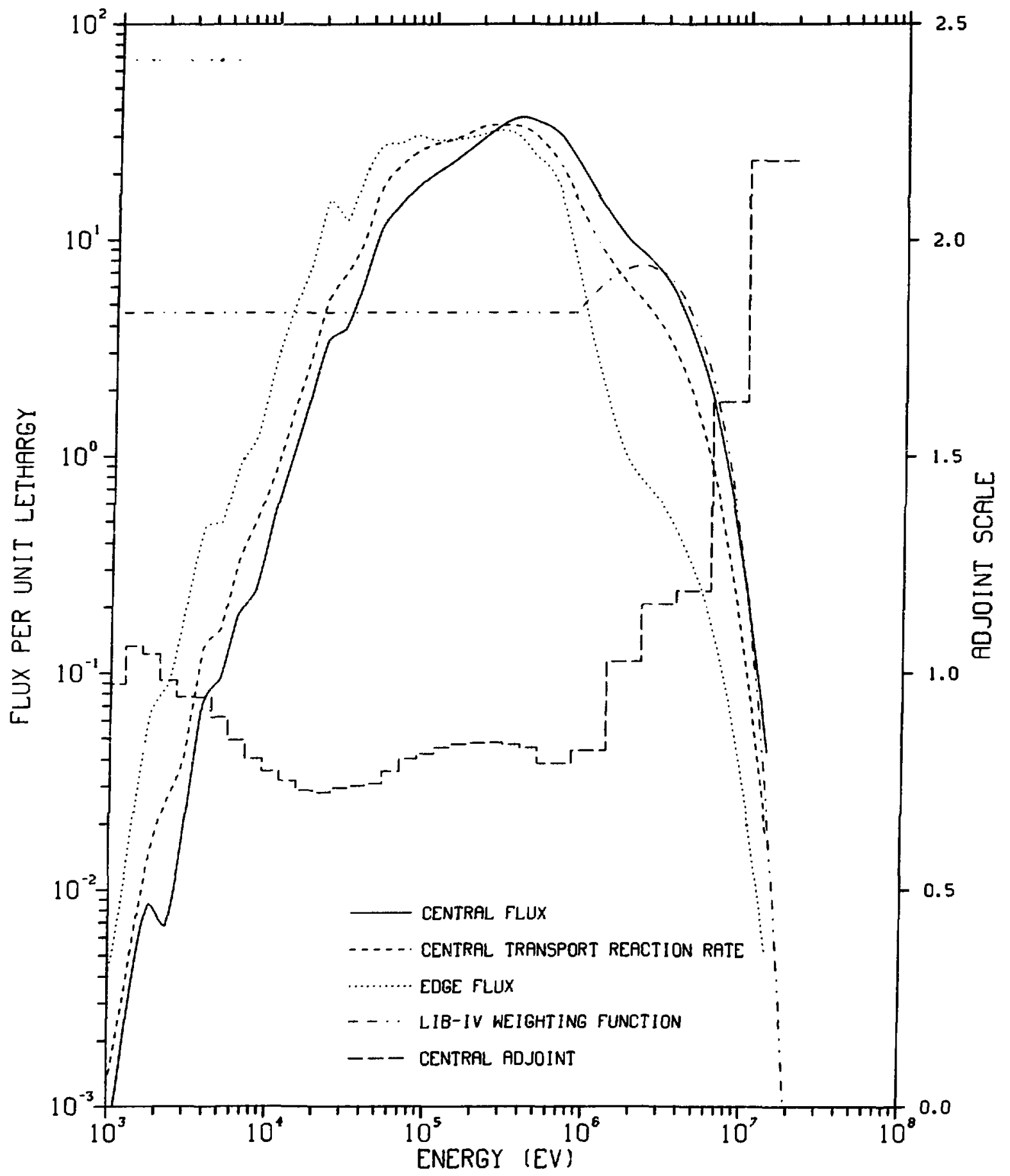

Fig. A-16. ZPR-3-11 spectra. 


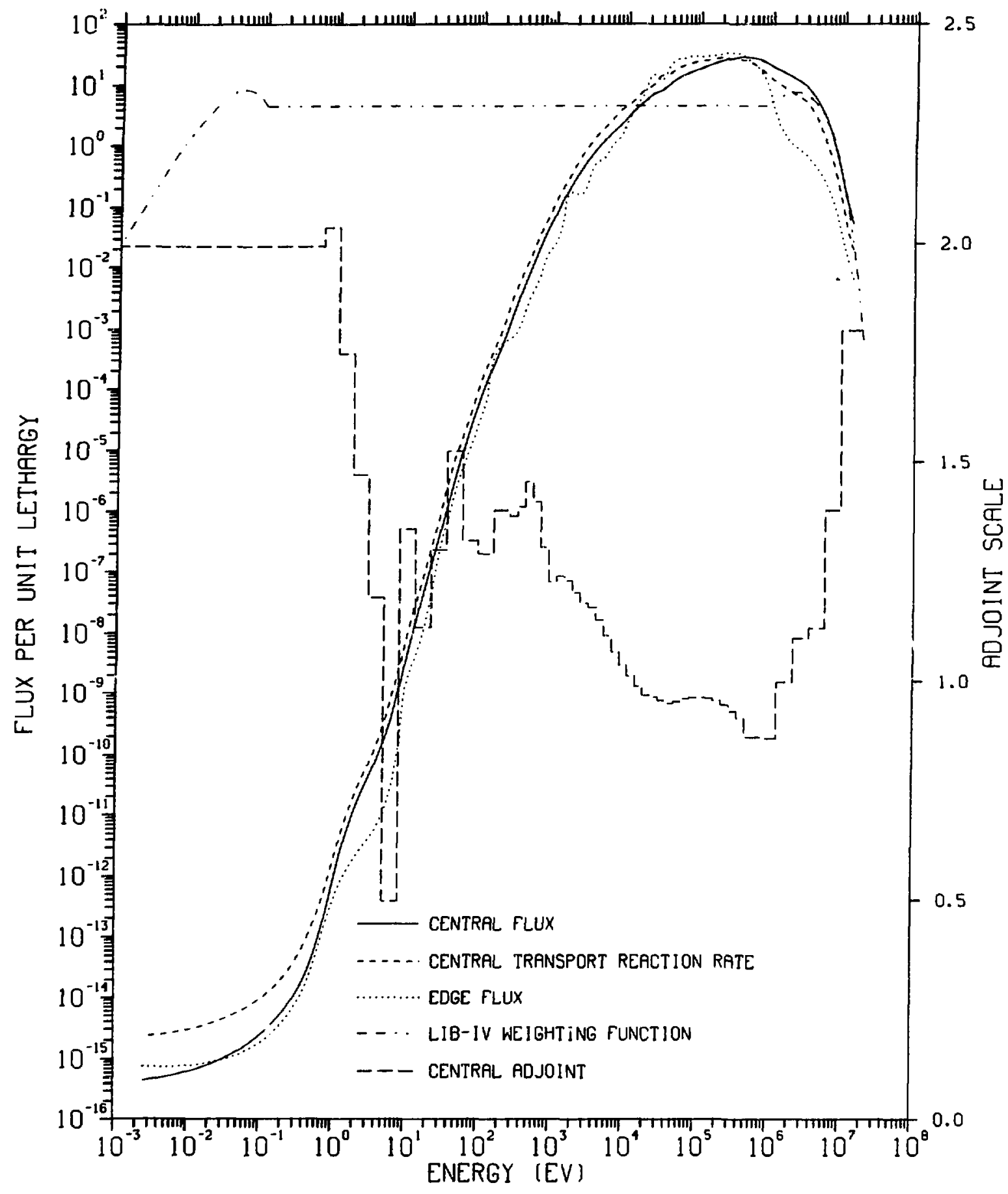

Fig. A-17. ZPR-3-12 spectra. 


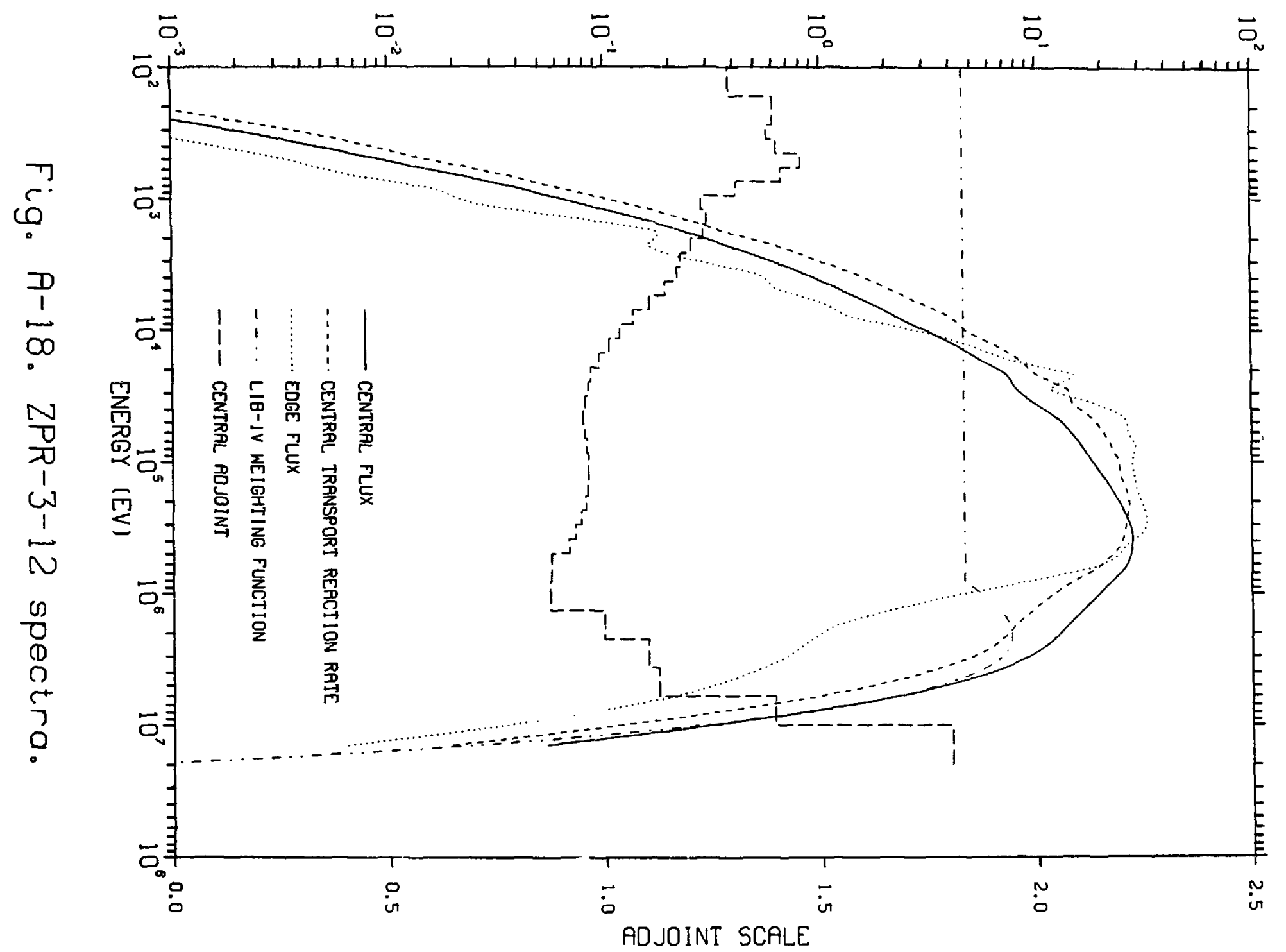




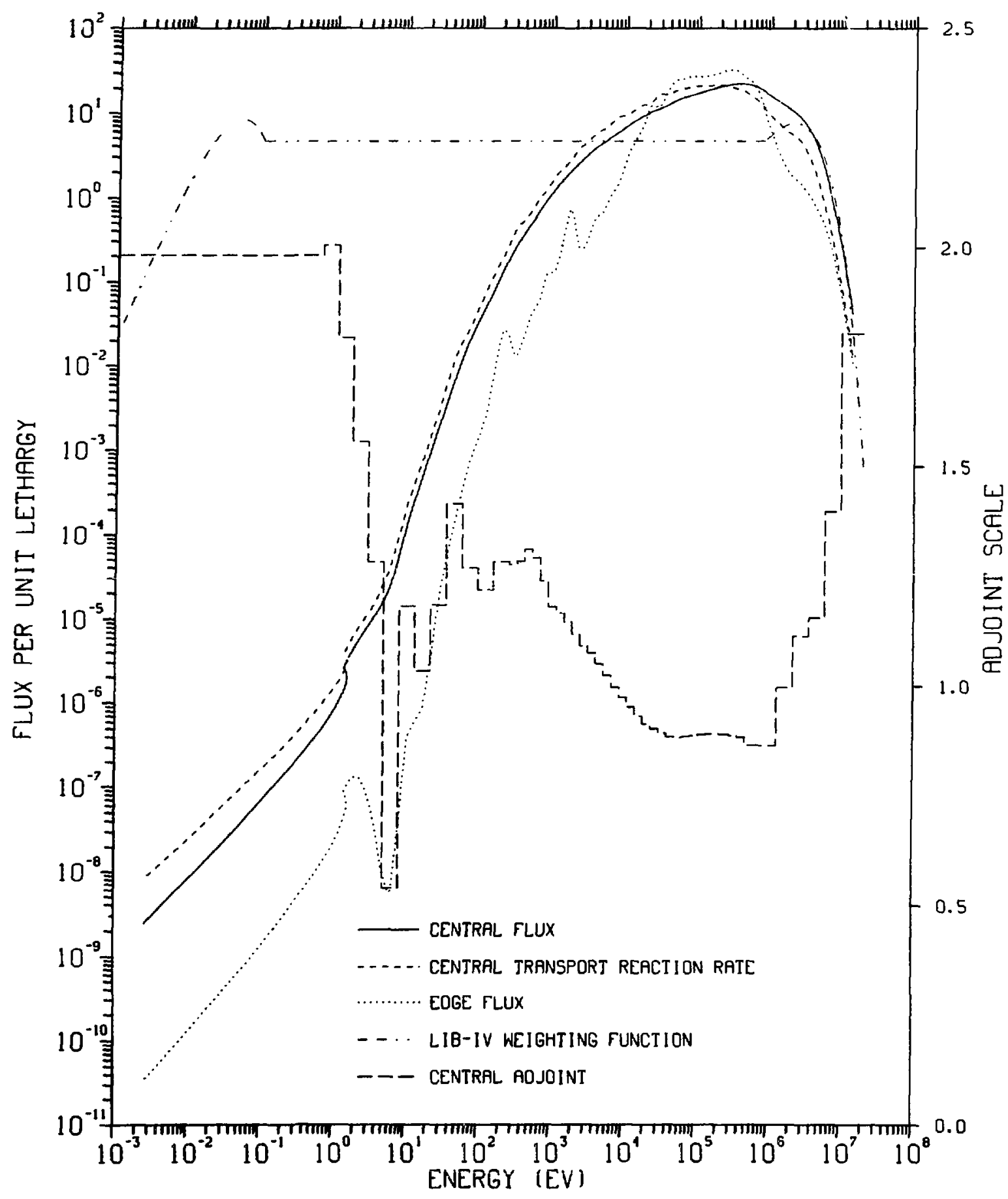

Fig. A-19. ZEBRA-2 spectra. 


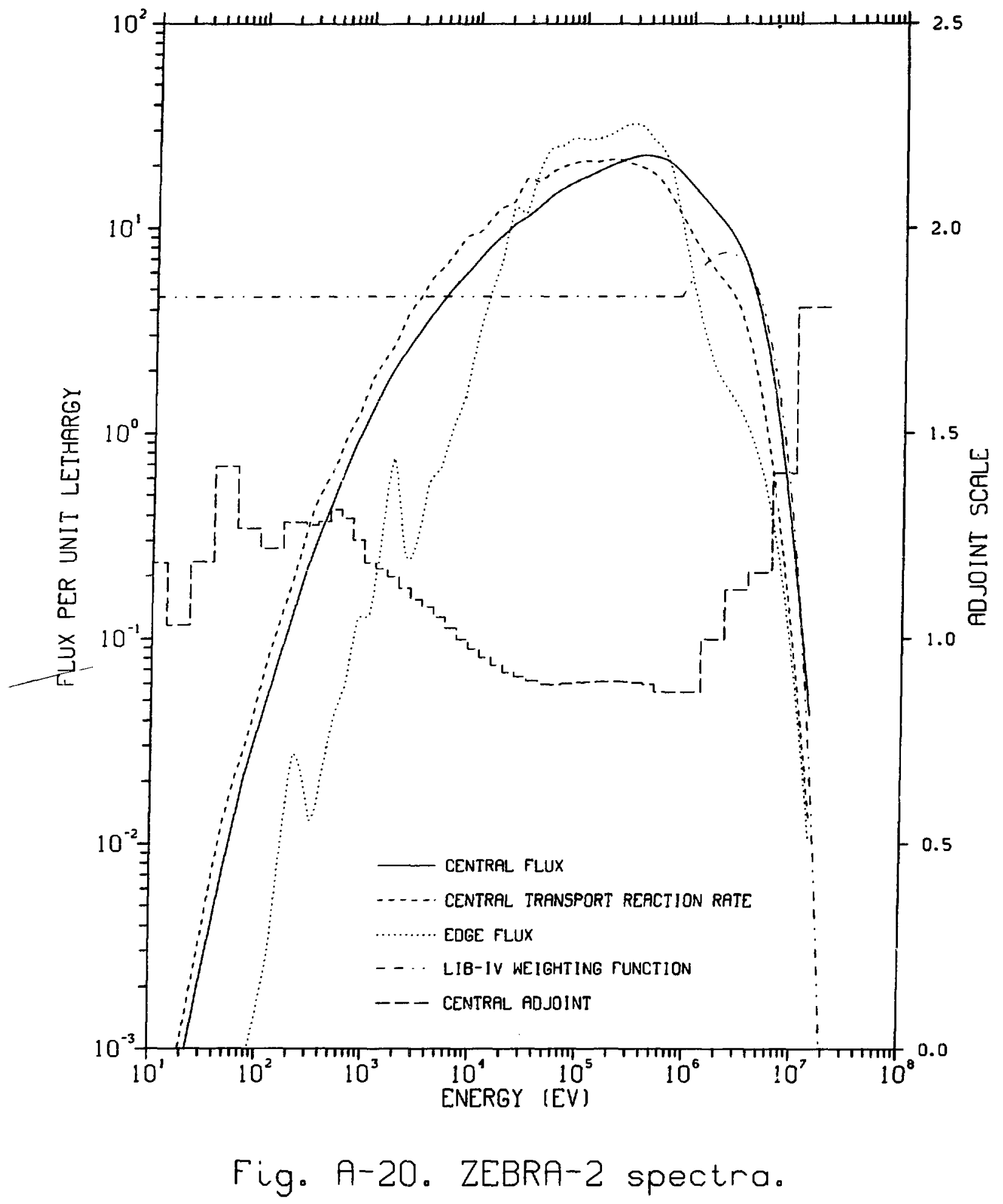




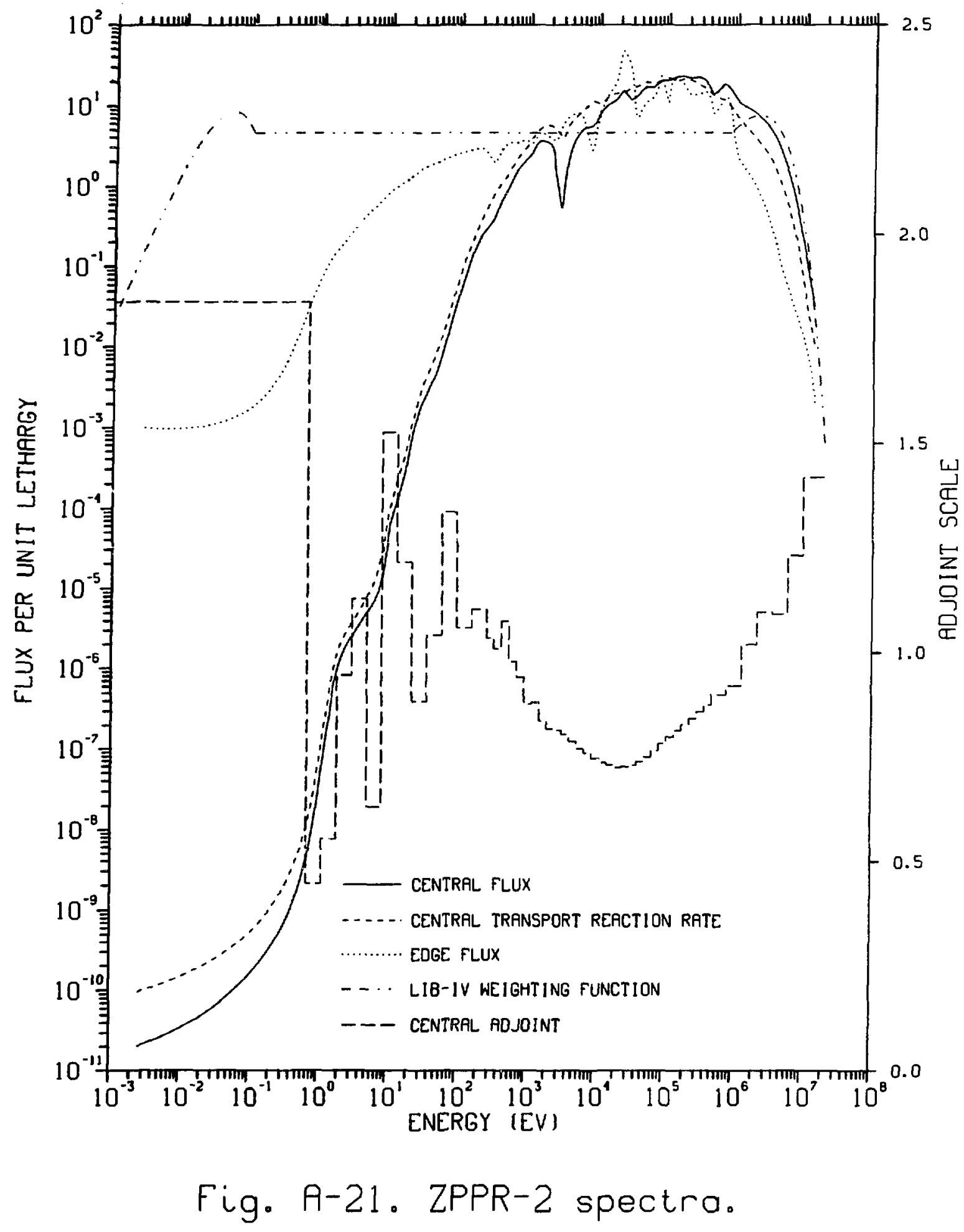




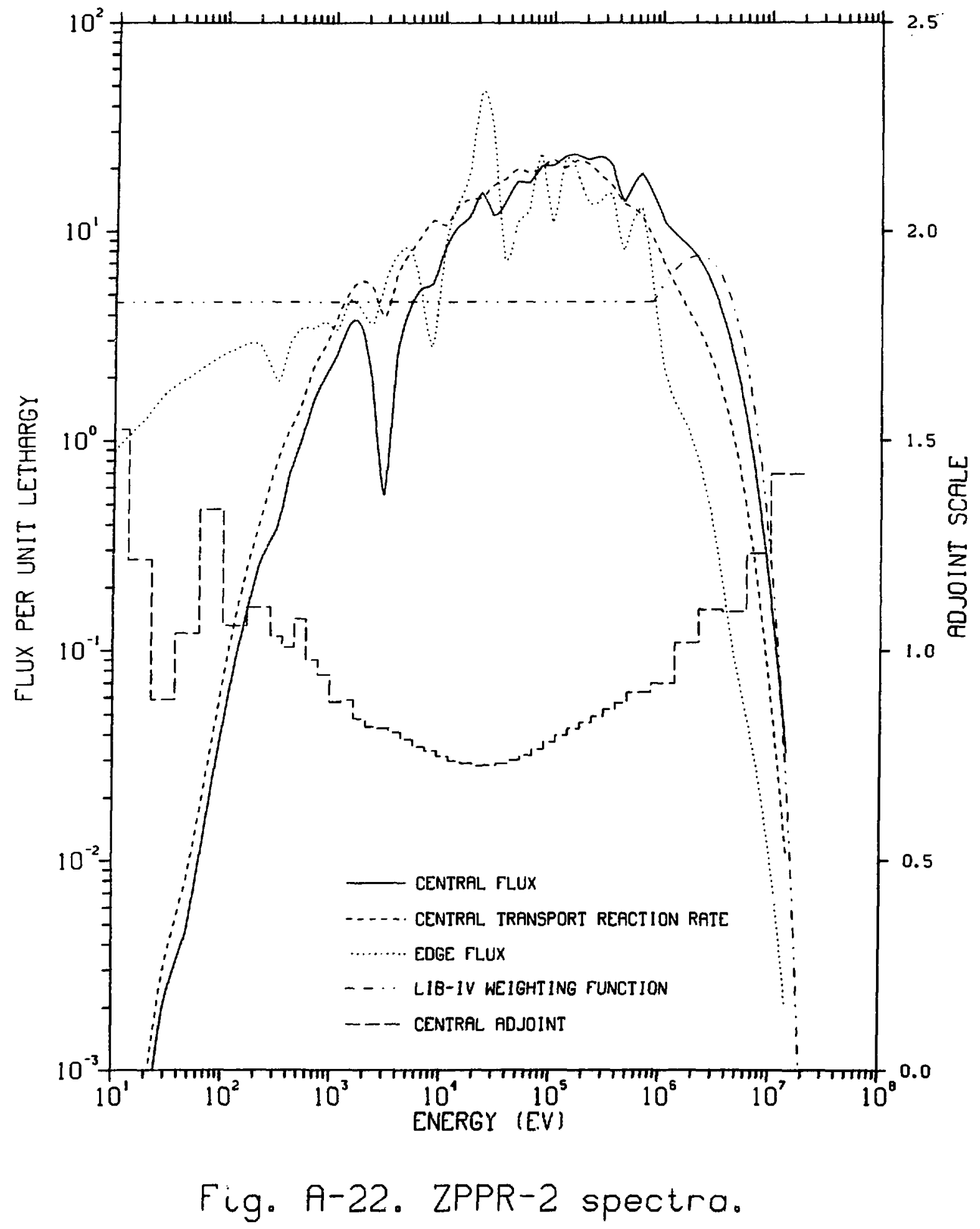




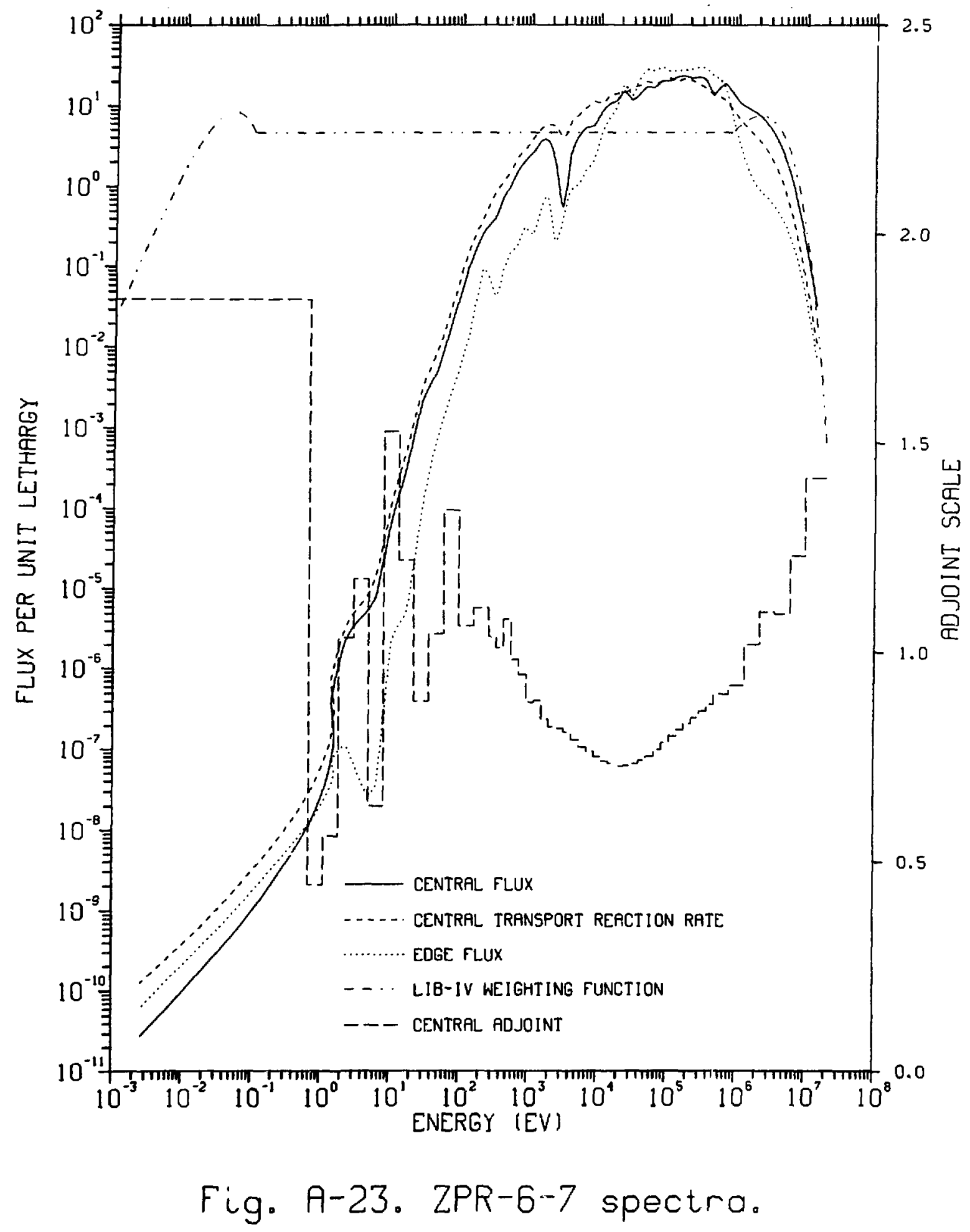




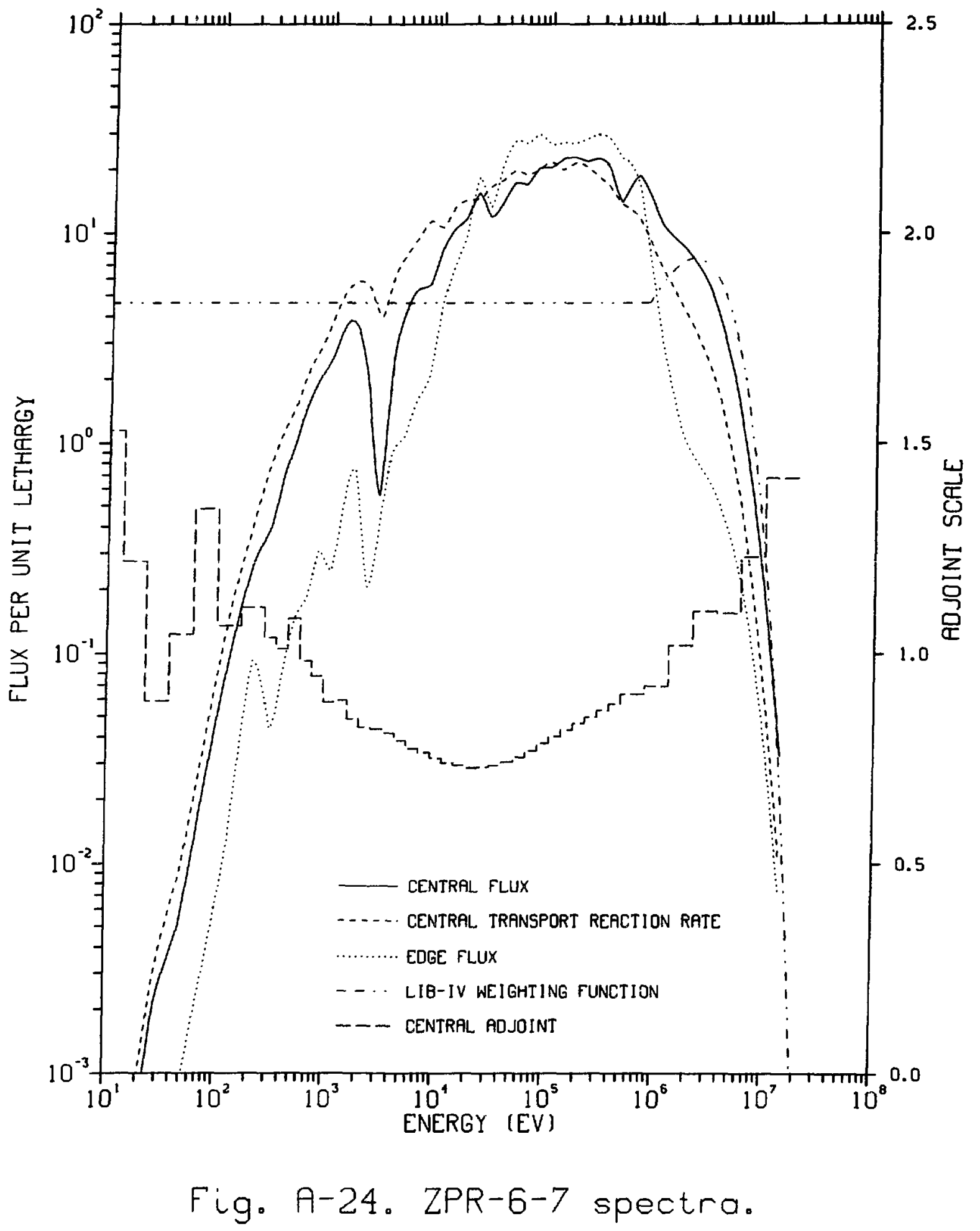




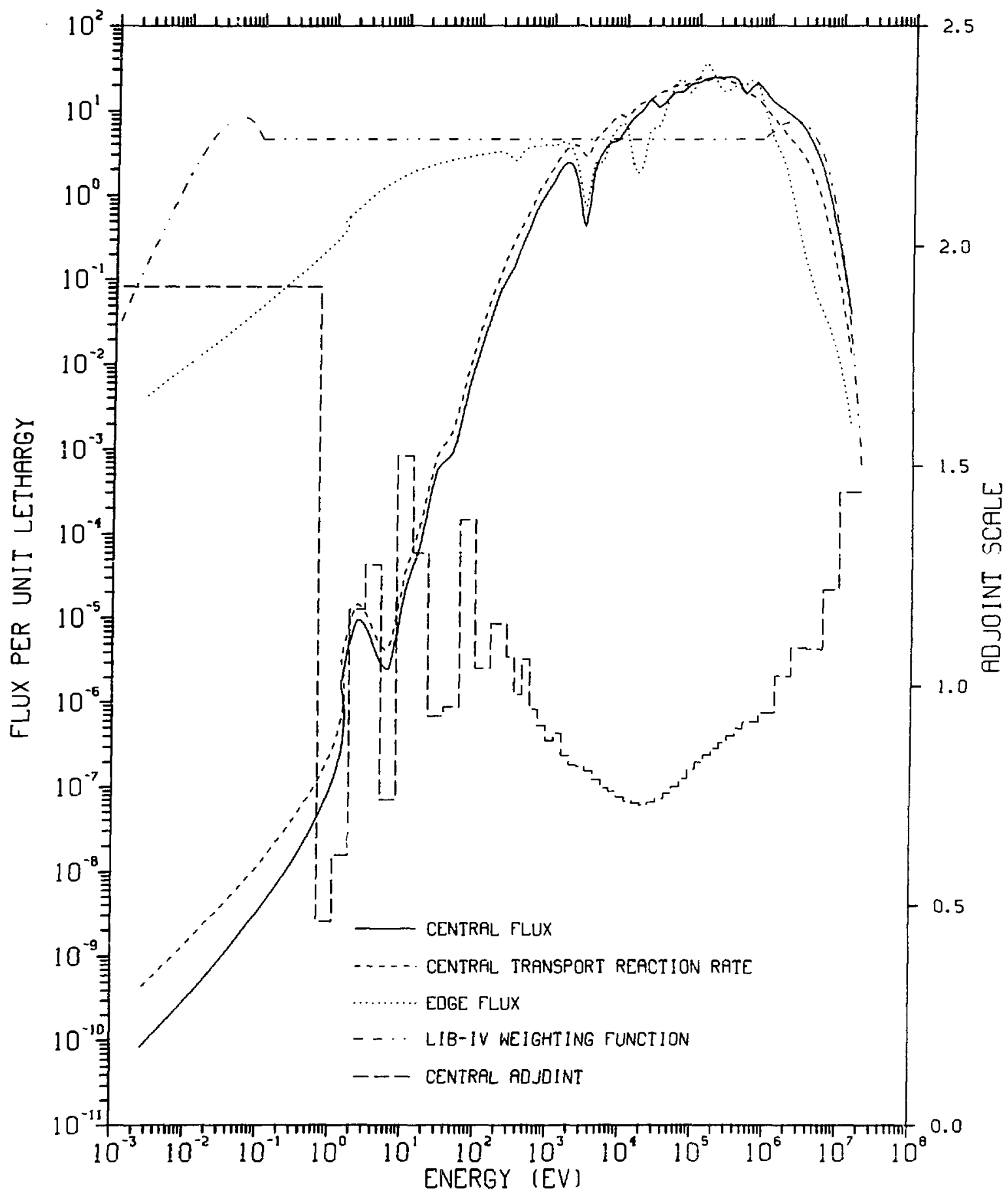

Fig. A-25. ZPR-3-56B spectra. 


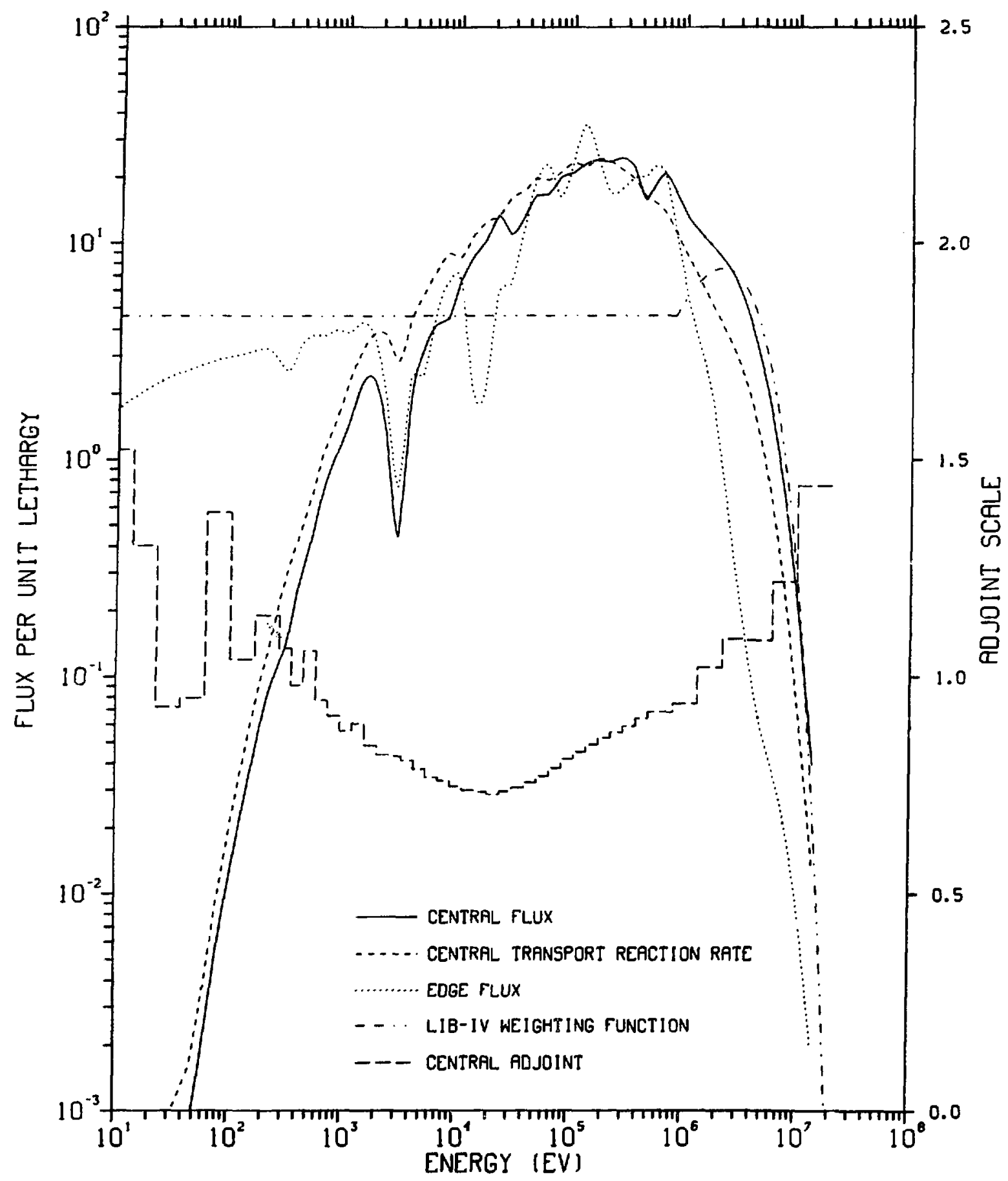

Fig. A-26. ZPR-3-56B spectra. 


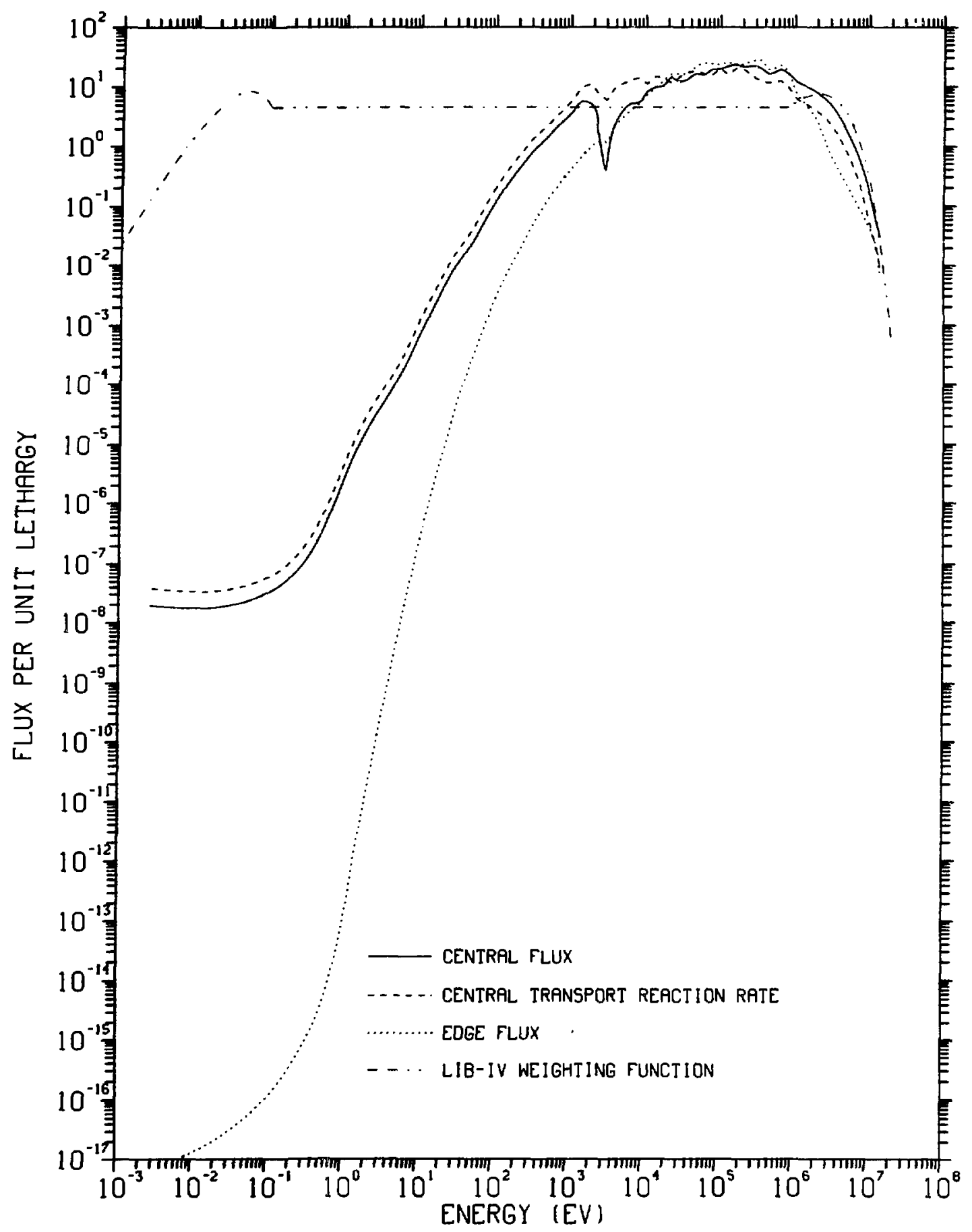

Fig. A-27. SEFOR spectra. 


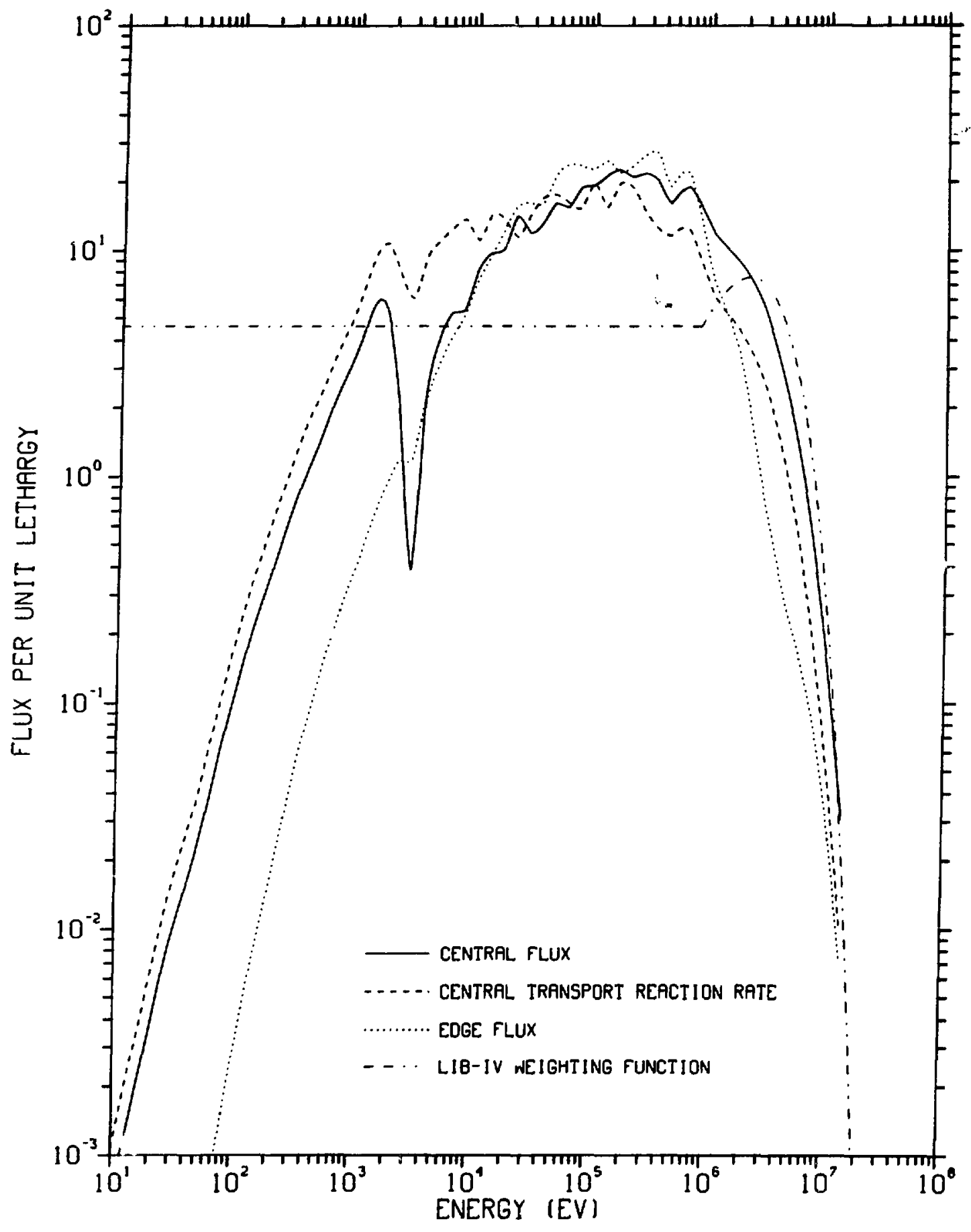

Fig. A-28. SEFOR spectra. 


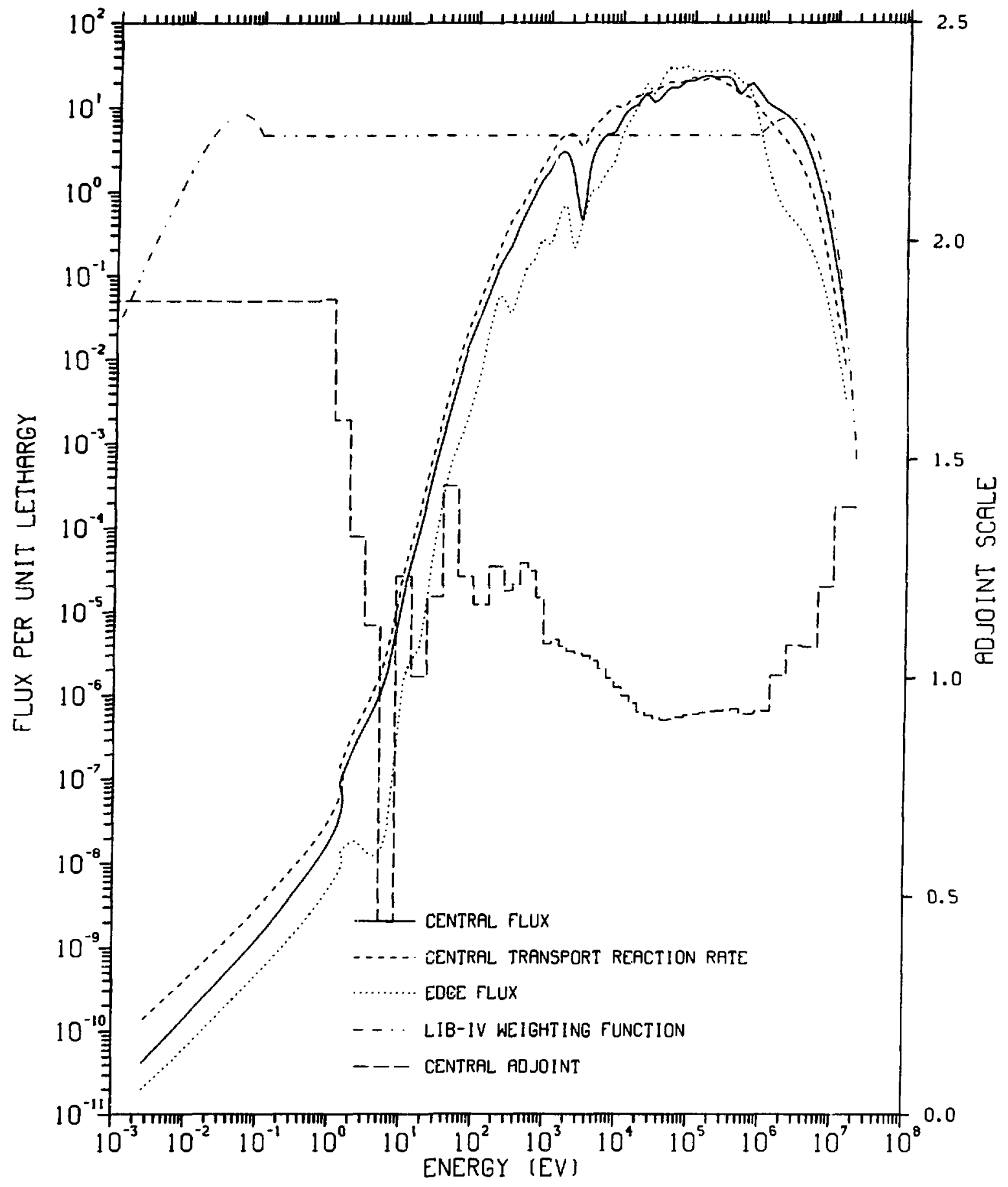

Fig. A-29. ZPR-6-6A spectra. 


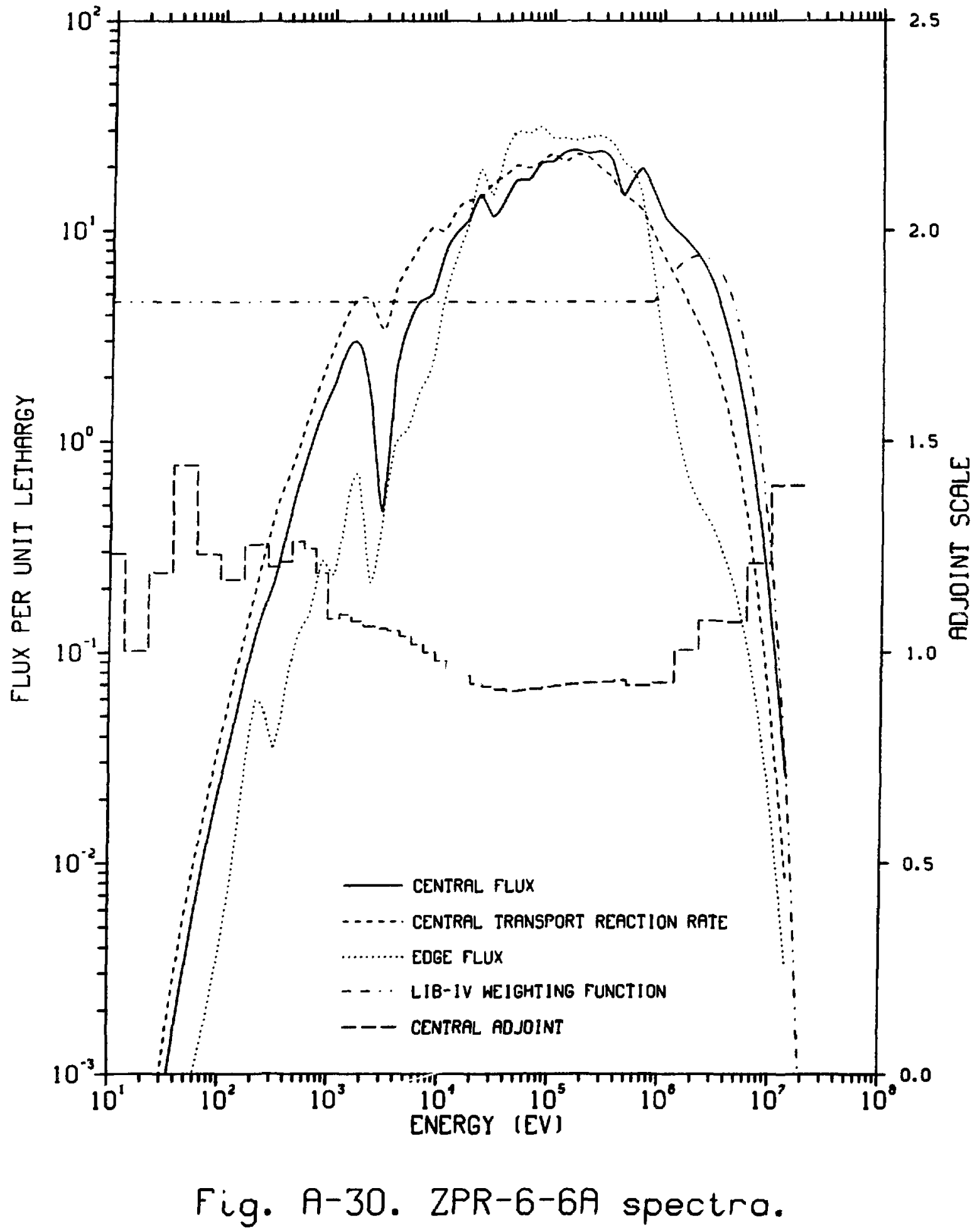




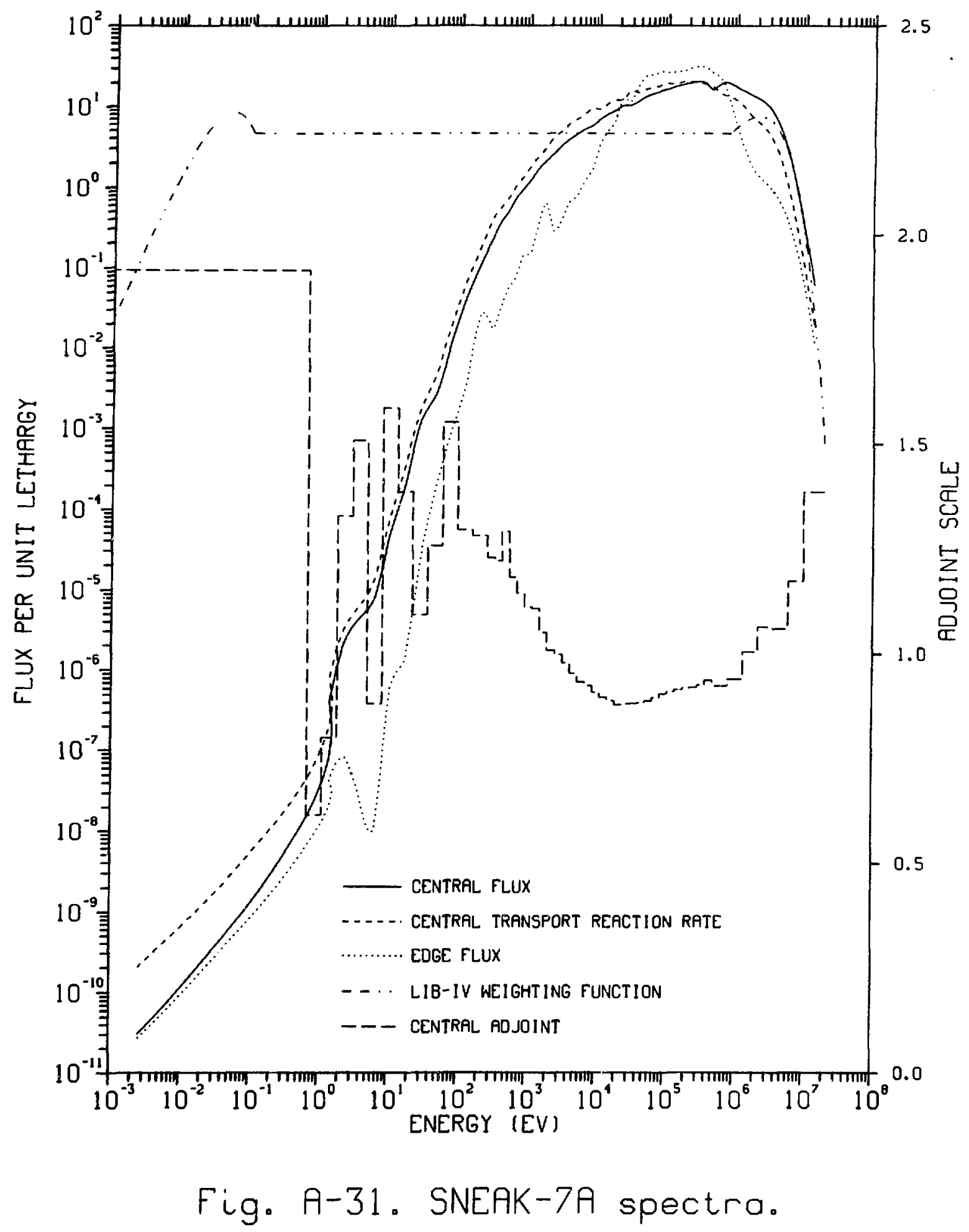




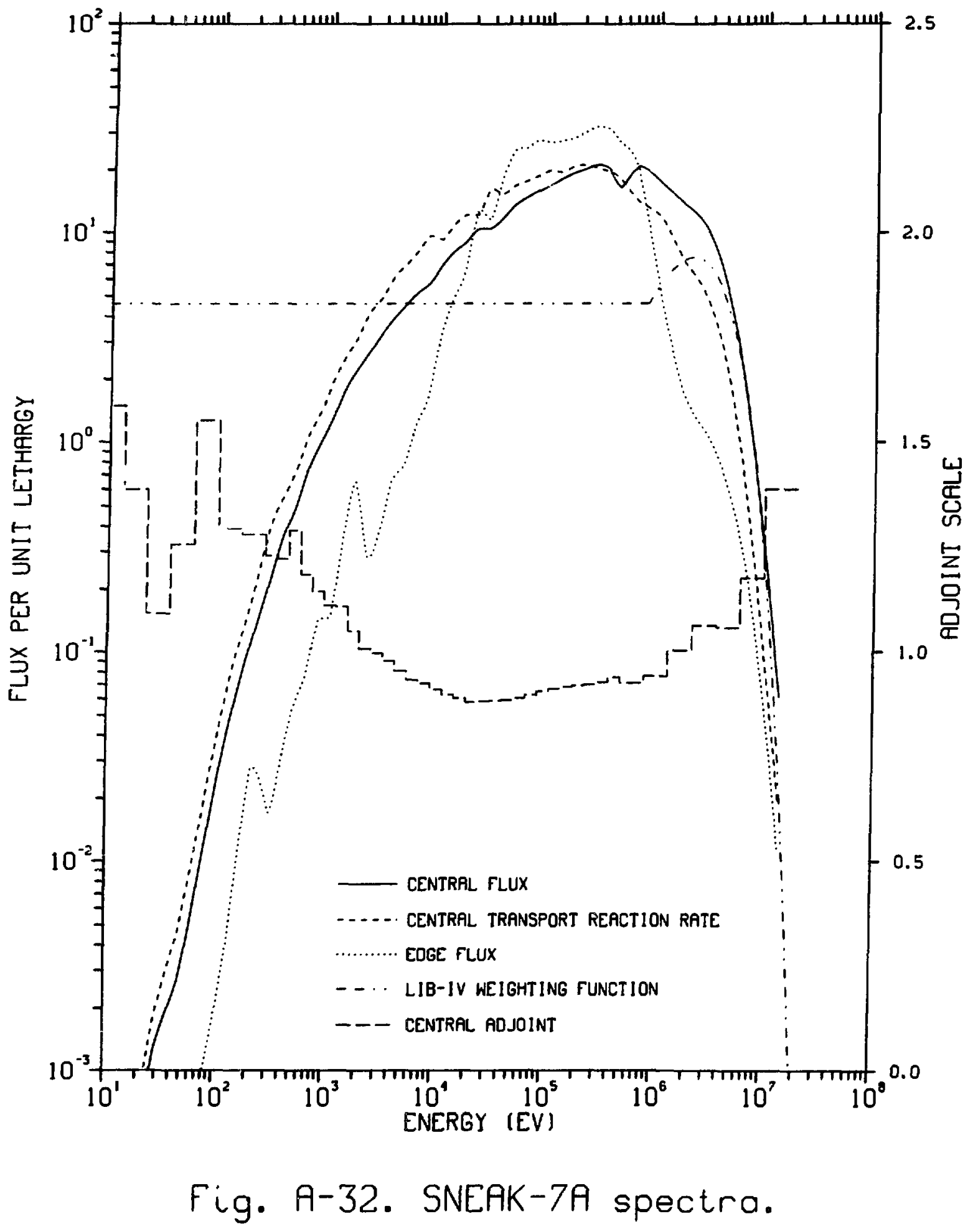




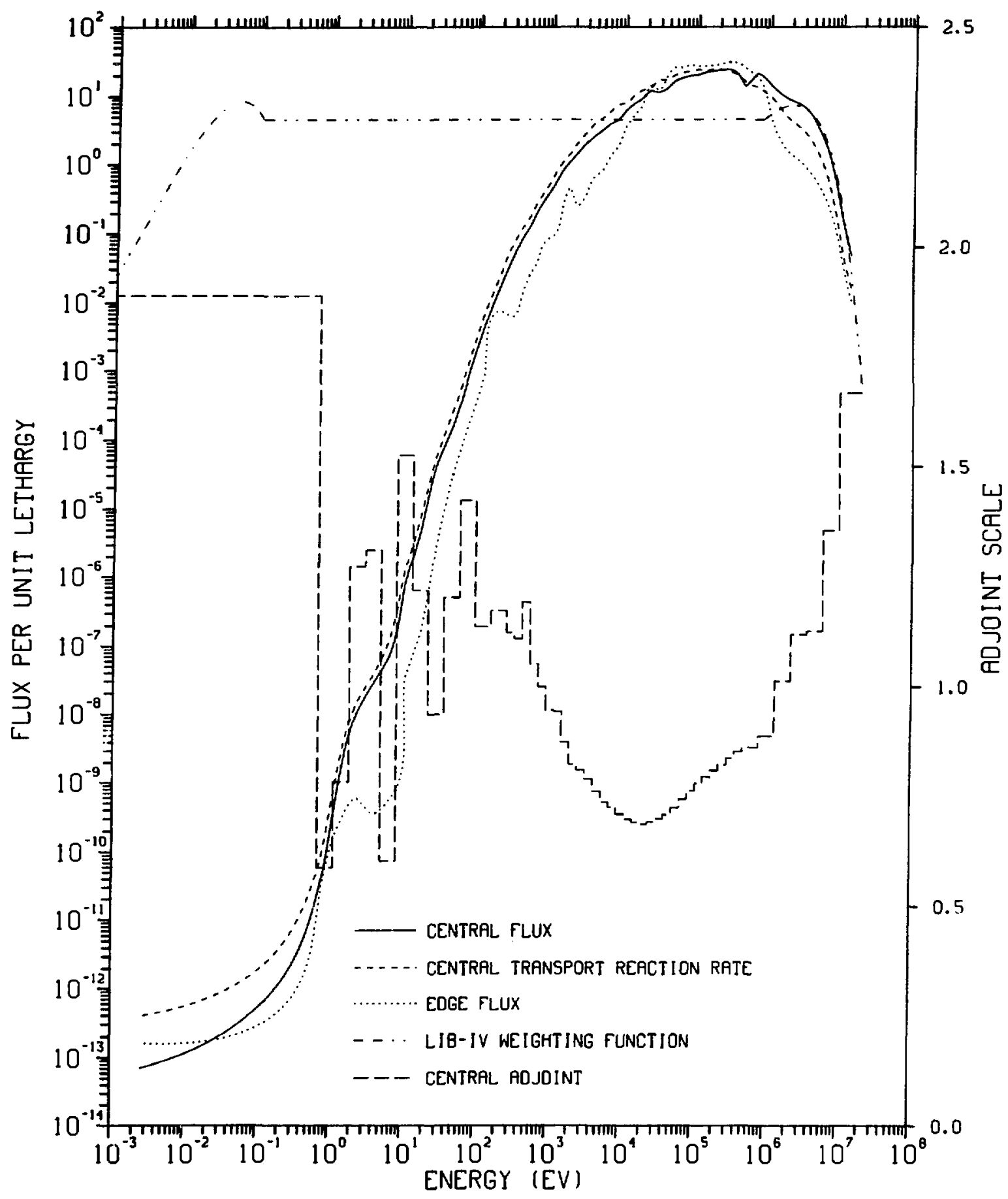

Fig. A-33. SNEAK-7B spectra. 


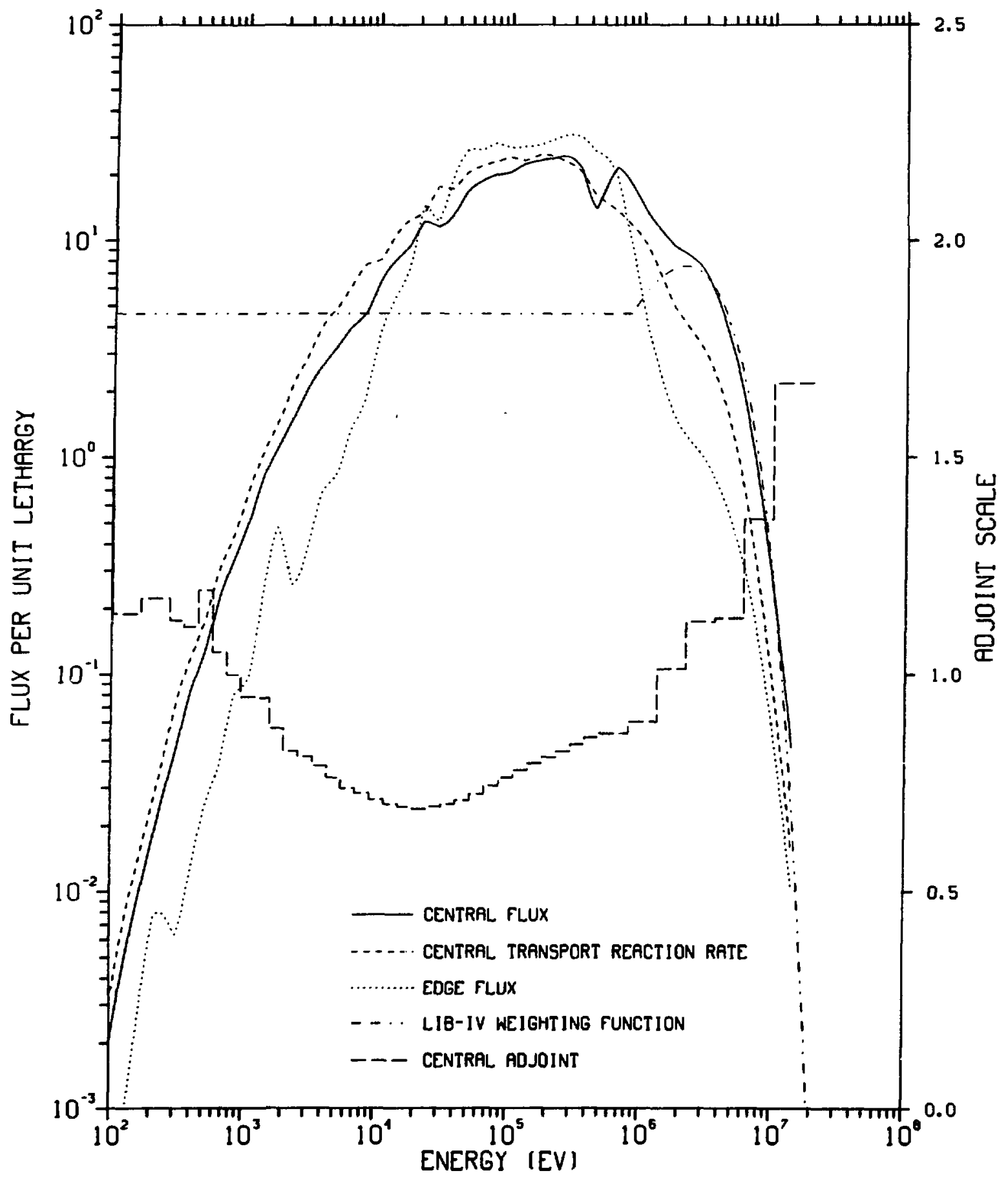

Fig. A-34. SNEAK-7B spectra. 
NUMERICAI DATA

This appendix contains the numerical spectra described in the text. There are three tables for each benchmark. The first table features the central flux spectrum, the second features the edge flux spectrum, and the third contains the central adjoint spectrum. Each table shows the spectrum as calculated in three different ways: with 1-D diffusion theory, with 1-D transport theory, and with 2-D diffusion theory.

The energy boundarles are given in units of electron volts (eV). The flux units are neutrons per square centimeter per second $\left(\mathrm{n} / \mathrm{cm}^{2} / \mathrm{s}\right)$. The adjoint units are proportional to reactivity gain per neutron insertion per second ( $\mathrm{rho} / \mathrm{n} / \mathrm{s}$ ). 
TABLE B-1

JEZEBEL CENTRAL FLUX SPECTRA

$\begin{array}{rrrr} & & \begin{array}{r}1-D \\ \text { ENERGY }\end{array} & \begin{array}{r}1-D \\ \text { DIFFUSION }\end{array} \\ \text { GROUP } & \text { TRANSPORT } \\ & \text { THEORY } & \text { THEORY } \\ \text { IN } / C M 2 / S \text { I } & \text { INVI } & \text { IN/CM2/SI } & \\ 1 & 1.9971 E+07 & 1.8763 E-01 & 2.0315 E-01 \\ 2 & 1.0000 E+07 & 2.3033 E+00 & 2.4375 E+00 \\ 3 & 6.0653 E+06 & 8.6792 E+00 & 8.9037 E+00 \\ 4 & 3.6788 E+06 & 1.5628 E+01 & 1.5783 E+01 \\ 5 & 2.2313 E+06 & 1.8436 E+01 & 1.8555 E+01 \\ 6 & 1.3534 E+06 & 1.7457 E+01 & 1.7608 E+01 \\ 7 & 8.2085 E+05 & 1.4251 E+01 & 1.4233 E+01 \\ 8 & 4.9787 E+05 & 5.5659 E+00 & 5.4899 E+00 \\ 9 & 3.8774 E+05 & 4.4882 E+00 & 4.3878 E+00 \\ 10 & 3.0197 E+05 & 3.4990 E+00 & 3.3937 E+00 \\ 11 & 2.3518 E+05 & 2.6630 E+00 & 2.5630 E+00 \\ 12 & 1.8316 E+05 & 2.0207 E+00 & 1.9265 E+00 \\ 13 & 1.4264 E+05 & 1.5360 E+00 & 1.4529 E+00 \\ 14 & 1.1109 E+05 & 1.1321 E+00 & 1.0631 E+00 \\ 15 & 8.6517 E+04 & 7.6106 E-01 & 7.0941 E-01 \\ 16 & 6.7379 E+04 & 4.8181 E-01 & 4.4570 E-01 \\ 17 & 5.2475 E+04 & 3.3246 E-01 & 3.0610 E-01 \\ 18 & 4.0868 E+04 & 2.0739 E-01 & 1.8955 E-01 \\ 19 & 3.1828 E+04 & 1.3168 E-01 & 1.1983 E-01 \\ 20 & 2.4788 E+04 & 8.0612 E-02 & 7.6793 E-02 \\ 21 & 1.9305 E+04 & 5.4468 E-02 & 5.2315 E-02 \\ 22 & 1.5034 E+04 & 3.6564 E-02 & 3.5185 E-02 \\ 23 & 1.1709 E+04 & 2.3032 E-02 & 2.2081 E-02 \\ 24 & 9.1188 E+03 & 1.5234 E-02 & 1.4645 E-02 \\ 25 & 7.1017 E+03 & 1.0052 E-02 & 9.7080 E-03 \\ 26 & 5.5308 E+03 & 6.6089 E-03 & 6.4154 E-03 \\ 27 & 4.3074 E+03 & 4.2402 E-03 & 4.1192 E-03 \\ 28 & 3.3546 E+03 & 2.5570 E-03 & 2.4821 E-03 \\ 29 & 2.6126 E+03 & 1.7946 E-03 & 1.7576 E-03 \\ 30 & 2.0347 E+03 & 1.2180 E-03 & 1.1856 E-03 \\ 31 & 1.5846 E+03 & 8.0717 E-04 & 7.7419 E-04 \\ 32 & 1.2341 E+03 & 4.6629 E-04 & 4.5950 E-04 \\ 33 & 9.6112 E+02 & 3.2398 E-04 & 3.1435 E-04 \\ 34 & 7.4852 E+02 & 2.5032 E-04 & 2.4490 E-04 \\ 35 & 5.8295 E+02 & 1.2008 E-04 & 1.2148 E-04 \\ 36 & 4.5400 E+02 & 7.5761 E-05 & 7.3007 E-05 \\ 37 & 3.5358 E+02 & 5.5298 E-05 & 5.3570 E-05 \\ 38 & 2.7536 E+02 & 4.2980 E-05 & 4.0727 E-05 \\ 39 & 1.6702 E+02 & 2.8450 E-07 & 2.6944 E-07 \\ 40 & 1.0130 E+02 & 9.9056 E-10 & 9.3957 E-10 \\ 41 & 6.1442 E+01 & 5.1158 E-12 & 4.9846 E-12 \\ 42 & 3.7267 E+01 & 4.0988 E-14 & 4.1433 E-14 \\ 43 & 2.2603 E+01 & 1.0899 E-16 & 1.1252 E-16 \\ 44 & 1.3710 E+01 & 3.0418 E-19 & 3.1763 E-19 \\ 45 & 8.3153 E+00 & 1.1218 E-21 & 1.1822 E-21 \\ 46 & 5.0435 E+00 & 5.8302 E-24 & 7.1621 E-24 \\ 47 & 3.0590 E+00 & 3.0071 E-26 & 3.1428 E-26 \\ 48 & 1.8554 E+00 & 5.2426 E-29 & 5.4874 E-29 \\ 43 & 1.1254 E+00 & 3.8119 E-32 & 3.9928 E-32 \\ 50 & 6.8256 E-01 & 1.2817 E-35 & 1.3426 E-35 \\ & 1.0000 E-05 & 1.0000 E+02 & 1.0000 E+02 \\ & & & \end{array}$


TABLE B-II

JEZEBEL EOGE FLUX SPECTRA

\begin{tabular}{|c|c|c|c|}
\hline GKOUP & $\begin{array}{r}\text { ENERGY } \\
\text { BOUNDARY } \\
\text { (EV) }\end{array}$ & $\begin{array}{r}1-0 \\
\text { DIFFUSIDN } \\
\text { THEORY } \\
\text { (N/C:iZ/S ) }\end{array}$ & $\begin{array}{r}1-D \\
\text { TRANSPORT } \\
\text { THEORY } \\
\text { (N/CM2/S) }\end{array}$ \\
\hline $\begin{array}{l}1 \\
2 \\
3 \\
4 \\
5 \\
6 \\
7 \\
8 \\
9 \\
10 \\
11 \\
12 \\
13 \\
14 \\
15 \\
16 \\
17 \\
18 \\
19 \\
20 \\
21 \\
22 \\
23 \\
24 \\
25 \\
26 \\
27 \\
28 \\
29 \\
30 \\
31 \\
32 \\
33 \\
34 \\
35 \\
36 \\
37 \\
38 \\
39 \\
40 \\
41 \\
42 \\
43 \\
44 \\
45 \\
46 \\
47 \\
48 \\
49 \\
50\end{array}$ & $\begin{array}{l}1.9971 E+07 \\
1.0000 E+07 \\
6.0653 E+06 \\
3.6788 E+06 \\
2.2313 E+06 \\
1.3534 E+06 \\
8.2085 E+05 \\
4.9787 E+05 \\
3.8774 E+05 \\
3.0197 E+05 \\
2.3518 E+05 \\
1.8316 E+05 \\
1.4264 E+05 \\
1.1109 E+05 \\
8.6517 E+04 \\
6.7379 E+04 \\
5.2475 E+04 \\
4.0868 E+04 \\
3.1828 E+04 \\
2.4788 E+04 \\
1.9305 E+04 \\
1.5034 E+04 \\
1.1709 E+04 \\
9.1188 E+03 \\
7.1017 E+03 \\
5.5308 E+03 \\
4.3074 E+03 \\
3.3546 E+03 \\
2.6126 E+03 \\
2.0347 E+03 \\
1.5846 E+03 \\
1.2341 E+03 \\
9.6112 E+02 \\
7.4852 E+02 \\
5.8295 E+02 \\
4.5400 E+02 \\
3.5358 E+02 \\
2.7536 E+02 \\
1.6702 E+02 \\
1.0130 E+02 \\
6.1442 E+01 \\
3.7267 E+01 \\
2.2603 E+01 \\
1.3710 E+01 \\
8.3153 E+00 \\
5.0435 E+00 \\
3.0590 E+00 \\
1.8554 E+00 \\
1.1254 E+00 \\
6.8256 E-01 \\
1.0000 E-05\end{array}$ & $\begin{array}{l}2.3823 E-01 \\
2.7792 E+00 \\
9.7425 E+00 \\
1.6794 E+01 \\
1.9390 E+01 \\
1.8046 E+01 \\
1.3898 E+01 \\
5.1055 E+00 \\
3.9465 E+00 \\
2.9593 E+00 \\
2.1677 E+00 \\
1.5659 E+00 \\
1.1365 E+00 \\
8.0361 E-01 \\
5.2074 E-01 \\
3.1962 E-01 \\
2.1484 E-01 \\
1.3132 E-01 \\
8.2450 E-02 \\
5.3779 E-02 \\
3.6412 E-02 \\
2.4358 E-02 \\
1.5294 E-02 \\
1.0131 E-02 \\
6.6956 E-03 \\
4.4175 E-03 \\
2.8565 E-03 \\
1.7105 E-03 \\
1.2267 E-03 \\
8.2723 E-04 \\
5.3960 E-04 \\
3.2825 E-04 \\
2.2247 E-04 \\
1.7446 E-04 \\
9.0792 E-05 \\
5.2167 E-05 \\
3.7995 E-05 \\
2.8410 E-05 \\
1.5557 E-07 \\
4.4760 E-10 \\
2.5398 E-12 \\
2.2124 E-14 \\
5.0286 E-17 \\
1.2530 E-19 \\
4.5200 E-22 \\
2.7029 E-24 \\
1.0368 E-26 \\
1.4847 E-29 \\
9.5964 E-33 \\
3.1501 E-36 \\
1.0000 E+02\end{array}$ & $\begin{array}{l}2.2772 \mathrm{E}-01 \\
2.6725 \mathrm{E}+00 \\
9.4516 \mathrm{E}+00 \\
1.6416 \mathrm{E}+01 \\
1.9106 \mathrm{E}+01 \\
1.7985 \mathrm{E}+01 \\
1.4104 \mathrm{E}+01 \\
5.2590 \mathrm{E}+00 \\
4.1012 \mathrm{E}+00 \\
3.0986 \mathrm{E}+00 \\
2.2855 \mathrm{E}+00 \\
1.6650 \mathrm{E}+00 \\
1.2179 \mathrm{E}+00 \\
8.6662 \mathrm{E}-01 \\
5.6389 \mathrm{E}-01 \\
3.4666 \mathrm{E}-01 \\
2.3370 \mathrm{E}-01 \\
1.4255 \mathrm{E}-01 \\
8.9173 \mathrm{E}-02 \\
5.6948 \mathrm{E}-02 \\
3.8520 \mathrm{E}-02 \\
2.5752 \mathrm{E}-02 \\
1.6104 \mathrm{E}-02 \\
1.0624 \mathrm{E}-02 \\
6.9876 \mathrm{E}-03 \\
4.5833 \mathrm{E}-03 \\
2.9466 \mathrm{E}-03 \\
1.7478 \mathrm{E}-03 \\
1.2467 \mathrm{E}-03 \\
8.4350 \mathrm{E}-04 \\
5.5301 \mathrm{E}-04 \\
3.2596 \mathrm{E}-04 \\
2.2441 \mathrm{E}-04 \\
1.7534 \mathrm{E}-04 \\
8.5875 \mathrm{E}-05 \\
5.2650 \mathrm{E}-05 \\
3.7096 \mathrm{E}-05 \\
2.8404 \mathrm{E}-05 \\
1.6013 \mathrm{E}-07 \\
4.7275 \mathrm{E}-10 \\
2.5124 \mathrm{E}-12 \\
2.4573 \mathrm{E}-14 \\
5.4782 \mathrm{E}-17 \\
1.4287 \mathrm{E}-19 \\
5.5247 \mathrm{E}-22 \\
3.5892 \mathrm{E}-24 \\
1.4404 \mathrm{E}-26 \\
2.0891 \mathrm{E}-29 \\
1.3340 \mathrm{E}-32 \\
4.2876 \mathrm{E}-36 \\
1.0000 \mathrm{E}+02\end{array}$ \\
\hline
\end{tabular}


TABLE B-III

JEZEBEL CENTRAL ADJOINT SPECTRA

\begin{tabular}{|c|c|c|c|}
\hline GROUP & $\begin{array}{r}\text { ENERGY } \\
\text { BOUNDRRY } \\
\text { (EV) }\end{array}$ & $\begin{array}{r}1-D \\
\text { DIFFUSION } \\
\text { THEORY } \\
\text { IRHO/N/SI }\end{array}$ & $\begin{array}{r}1-D \\
\text { TRANSPORT } \\
\text { THEORY } \\
\text { (RHO/N/S) }\end{array}$ \\
\hline $\begin{array}{r}1 \\
2 \\
3 \\
4 \\
5 \\
6 \\
7 \\
8 \\
9 \\
10 \\
11 \\
12 \\
13 \\
14 \\
15 \\
16 \\
17 \\
18 \\
19 \\
20 \\
21 \\
22 \\
23 \\
24 \\
25 \\
26 \\
27 \\
28 \\
29 \\
30 \\
31 \\
32 \\
33 \\
34 \\
35 \\
36 \\
37 \\
38 \\
39 \\
40 \\
41 \\
42 \\
43 \\
44 \\
45 \\
46 \\
47 \\
48 \\
49 \\
50\end{array}$ & $\begin{array}{l}1.9971 E+07 \\
1.0000 E+07 \\
6.0653 E+06 \\
3.6788 E+06 \\
2.2313 E+06 \\
1.3534 E+06 \\
8.2085 E+05 \\
4.9787 E+05 \\
3.8774 E+05 \\
3.0197 E+05 \\
2.3518 E+05 \\
1.8316 E+05 \\
1.4264 E+05 \\
1.1109 E+05 \\
8.6517 E+04 \\
6.7379 E+04 \\
5.2475 E+04 \\
4.0868 E+04 \\
3.1828 E+04 \\
2.4788 E+04 \\
1.9305 E+04 \\
1.5034 E+04 \\
1.1709 E+04 \\
9.1188 E+03 \\
7.1017 E+03 \\
5.5308 E+03 \\
4.3074 E+03 \\
3.3546 E+03 \\
2.6126 E+03 \\
2.0347 E+03 \\
1.5846 E+03 \\
1.2341 E+03 \\
9.6112 E+02 \\
7.4852 E+02 \\
5.8295 E+02 \\
4.5400 E+02 \\
3.5358 E+02 \\
2.7536 E+02 \\
1.6702 E+02 \\
1.0130 E+02 \\
6.1442 E+01 \\
3.7267 E+01 \\
2.2603 E+01 \\
1.3710 E+01 \\
8.3153 E+00 \\
5.0435 E+00 \\
3.0590 E+00 \\
1.8554 E+00 \\
1.1254 E+00 \\
6.8256 E-01 \\
1.0000 E-05\end{array}$ & $\begin{array}{l}1.4787 E+00 \\
1.2279 E+00 \\
1.0123 E+00 \\
1.0318 E+00 \\
1.0207 E+00 \\
9.5194 E-01 \\
9.2211 E-01 \\
9.2239 E-01 \\
9.2980 E-01 \\
9.3514 E-01 \\
9.5404 E-01 \\
9.9725 E-01 \\
1.0562 E+00 \\
1.0850 E+00 \\
1.1078 E+00 \\
1.1231 E+00 \\
1.1226 E+00 \\
1.1392 E+00 \\
1.1317 E+00 \\
1.0446 E+00 \\
1.0247 E+00 \\
1.0194 E+00 \\
1.0311 E+00 \\
1.0617 E+00 \\
9.9921 E-01 \\
1.0090 E+00 \\
1.0736 E+00 \\
1.1853 E+00 \\
1.0137 E+00 \\
1.0665 E+00 \\
1.3639 E+00 \\
1.3750 E+00 \\
1.3177 E+00 \\
1.1828 E+00 \\
1.6728 E+00 \\
1.5035 E+00 \\
1.4968 E+00 \\
1.6185 E+00 \\
1.6989 E+00 \\
2.2248 E+00 \\
1.4832 E+00 \\
1.3083 E+00 \\
1.9241 E+00 \\
1.9246 E+00 \\
2.0721 E+00 \\
2.4511 E+00 \\
2.3577 E+00 \\
1.4069 E+00 \\
8.8686 E-01 \\
2.0502 E+00 \\
6.4998 E+01\end{array}$ & $\begin{array}{l}1.5934 E+00 \\
1.2958 E+00 \\
1.0408 E+00 \\
1.0432 E+00 \\
1.0241 E+00 \\
9.5070 E-01 \\
9.0474 E-01 \\
8.8942 E-01 \\
8.8685 E-01 \\
8.8377 E-01 \\
8.9390 E-01 \\
9.2467 E-01 \\
9.7029 E-01 \\
9.9022 E-01 \\
1.0055 E+00 \\
1.0152 E+00 \\
1.0117 E+00 \\
1.0250 E+00 \\
1.0220 E+00 \\
9.8845 E-01 \\
9.7435 E-01 \\
9.6961 E-01 \\
9.7885 E-01 \\
1.0104 E+00 \\
9.5585 E-01 \\
9.6995 E-01 \\
1.0331 E+00 \\
1.1408 E+00 \\
9.8428 E-01 \\
1.0285 E+00 \\
1.2985 E+00 \\
1.3447 E+00 \\
1.2690 E+00 \\
1.1506 E+00 \\
1.6790 E+00 \\
1.4374 E+00 \\
1.4415 E+00 \\
1.5265 E+00 \\
1.6048 E+00 \\
2.1114 E+00 \\
1.4274 E+00 \\
1.2299 E+00 \\
1.8389 E+00 \\
1.8278 E+00 \\
1.9449 E+00 \\
2.2458 E+00 \\
2.19618 E+00 \\
1.3348 E+00 \\
8.4258 E-01 \\
1.9457 E+00 \\
6.2103 E+01\end{array}$ \\
\hline
\end{tabular}


TABLE B-IV

VERA-11A CENTRAL FLUX SPECTRA

\begin{tabular}{|c|c|c|c|c|}
\hline GROUP & $\begin{array}{r}\text { ENERGY } \\
\text { BOUNDARY } \\
\text { (EV) }\end{array}$ & $\begin{array}{r}1-0 \\
\text { OIFFUSION } \\
\text { THEORY } \\
\text { IN/CM2/SI }\end{array}$ & $\begin{array}{r}1-0 \\
\text { TRANSPORT } \\
\text { THEORY } \\
\text { (N/CM } 2 / S)\end{array}$ & $\begin{array}{r}2-0 \\
\text { DIFFUSION } \\
\text { THEORY } \\
\text { (N/CMZS/S) }\end{array}$ \\
\hline $\begin{array}{r}1 \\
2 \\
3 \\
4 \\
5 \\
6 \\
7 \\
8 \\
9 \\
10 \\
11 \\
12 \\
13 \\
14 \\
15 \\
16 \\
17 \\
18 \\
19 \\
20 \\
21 \\
22 \\
23 \\
24 \\
25 \\
26 \\
27 \\
28 \\
29 \\
30 \\
31 \\
32 \\
33 \\
34 \\
35 \\
36 \\
37 \\
38 \\
39 \\
40 \\
41 \\
42 \\
43 \\
44 \\
45 \\
46 \\
47 \\
48 \\
49 \\
50\end{array}$ & $\begin{array}{l}1.9971 E+07 \\
1.0000 E+07 \\
6.0653 E+06 \\
3.6788 E+06 \\
2.2313 E+06 \\
1.3534 E+06 \\
8.2085 E+05 \\
4.9787 E+05 \\
3.8774 E+05 \\
3.0197 E+05 \\
2.3518 E+05 \\
1.8316 E+05 \\
1.4264 E+05 \\
1.1109 E+05 \\
8.6517 E+04 \\
6.7379 E+04 \\
5.2475 E+04 \\
4.0368 E+04 \\
3.1828 E+04 \\
2.4788 E+04 \\
1.9305 E+04 \\
1.5034 E+04 \\
1.1709 E+04 \\
9.1188 E+03 \\
7.1017 E+03 \\
5.5308 E+03 \\
4.3074 E+03 \\
3.3546 E+03 \\
2.6126 E+03 \\
2.0347 E+03 \\
1.5846 E+03 \\
1.2341 E+03 \\
9.6112 E+02 \\
7.4852 E+02 \\
5.8295 E+02 \\
4.5400 E+02 \\
3.5358 E+02 \\
2.7536 E+02 \\
1.6702 E+02 \\
1.0130 E+02 \\
6.1442 E+01 \\
3.7267 E+01 \\
2.2603 E+01 \\
1.3710 E+01 \\
8.3153 E+00 \\
5.0435 E+00 \\
3.0590 E+00 \\
1.8554 E+00 \\
1.1254 E+00 \\
6.8256 E-01 \\
1.0000 E-05\end{array}$ & $\begin{array}{l}7.1900 E-02 \\
8.0021 E-01 \\
3.7532 E+00 \\
8.3851 E+00 \\
1.1323 E+01 \\
1.2190 E+01 \\
1.2274 E+01 \\
5.8597 E+00 \\
5.5170 E+00 \\
5.0626 E+00 \\
4.5927 E+00 \\
4.1685 E+00 \\
3.7453 E+00 \\
3.3064 E+00 \\
2.9429 E+00 \\
2.6098 E+00 \\
2.2985 E+00 \\
1.9090 E+00 \\
1.6257 E+00 \\
1.5203 E+00 \\
1.2679 E+00 \\
1.0653 E+00 \\
8.5830 E-01 \\
6.8785 E-01 \\
5.6846 E-01 \\
4.4411 E-01 \\
3.4834 E-01 \\
2.4908 E-01 \\
1.7687 E-01 \\
1.3322 E-01 \\
9.3312 E-02 \\
5.8678 E-02 \\
3.8213 E-02 \\
2.4739 E-02 \\
1.2956 E-02 \\
8.2932 E-03 \\
4.4877 E-03 \\
3.0931 E-03 \\
8.3912 E-04 \\
1.4465 E-04 \\
2.5190 E-05 \\
1.0782 E-05 \\
1.5538 E-06 \\
2.4662 E-07 \\
5.9083 E-08 \\
2.3191 E-08 \\
7.0328 E-09 \\
8.3029 E-10 \\
3.2743 E-11 \\
4.4542 E-13 \\
1.0000 E+02\end{array}$ & $\begin{array}{l}7.9041 E-02 \\
8.5282 E-01 \\
3.9184 E+00 \\
8.6096 E+00 \\
1.1772 E+01 \\
1.2567 E+01 \\
1.2429 E+01 \\
5.8813 E+00 \\
5.4911 E+00 \\
4.9964 E+00 \\
4.4981 E+00 \\
4.0549 E+00 \\
3.6238 E+00 \\
3.1834 E+00 \\
2.8236 E+00 \\
2.4930 E+00 \\
2.1852 E+00 \\
1.8056 E+00 \\
1.5365 E+00 \\
1.4340 E+00 \\
1.1954 E+00 \\
1.0048 E+00 \\
8.1101 E-0 \\
6.5173 E-0 \\
5.4155 E-01 \\
4.2630 E-01 \\
3.3666 E-01 \\
2.4224 E-01 \\
1.7407 E-01 \\
1.3261 E-01 \\
9.3610 E-02 \\
5.9431 E-02 \\
3.9098 E-02 \\
2.5576 E-02 \\
1.3565 E-02 \\
8.7706 E-03 \\
4.7932 E-03 \\
3.3411 E-03 \\
9.1703 E-04 \\
1.5939 E-04 \\
2.9196 E-05 \\
1.2197 E-05 \\
1.7768 E-06 \\
2.8470 E-07 \\
6.9040 E-08 \\
2.7299 E-08 \\
8.3243 E-09 \\
9.8646 E-10 \\
3.8978 E-11 \\
5.3036 E-13 \\
1.0000 E+02\end{array}$ & $\begin{array}{l}7.1681 E-02 \\
7.9765 E-01 \\
3.7383 E+00 \\
8.3457 E+00 \\
1.1276 E+01 \\
1.2153 E+01 \\
1.2265 E+01 \\
5.8616 E+00 \\
5.5244 E+00 \\
5.0730 E+00 \\
4.6041 E+00 \\
4.1802 E+00 \\
3.7568 E+00 \\
3.3174 E+00 \\
2.9537 E+00 \\
2.6201 E+00 \\
2.3082 E+00 \\
1.9169 E+00 \\
1.6320 E+00 \\
1.5257 E+00 \\
1.2722 E+00 \\
1.0687 E+00 \\
8.6081 E-01 \\
6.8974 E-01 \\
5.7167 E-01 \\
4.4776 E-01 \\
3.5207 E-01 \\
2.5233 E-01 \\
1.7957 E-01 \\
1.3550 E-11 \\
9.4891 E-02 \\
5.9655 E-02 \\
3.8840 E-02 \\
2.5138 E-02 \\
1.3163 E-02 \\
8.4247 E-03 \\
4.5588 E-03 \\
3.1416 E-03 \\
8.5211 E-04 \\
1.4686 E-04 \\
2.6583 E-05 \\
1.0932 E-05 \\
1.5749 E-06 \\
2.4984 E-07 \\
5.9800 E-08 \\
2.3439 E-08 \\
7.1020 E-09 \\
8.3828 E-10 \\
3.3057 E-11 \\
4.4968 E-13 \\
1.0000 E+02\end{array}$ \\
\hline
\end{tabular}


TABLE $B-V$

VERA-11A EDGE FLUX SPECTRA

\begin{tabular}{|c|c|c|c|c|}
\hline GROUP & $\begin{array}{r}\text { ENERGY } \\
\text { BOUNDRRY } \\
\text { (EV! }\end{array}$ & $\begin{array}{r}1-0 \\
\text { DIFFUSION } \\
\text { THEORY } \\
\text { (N/CM2/S) }\end{array}$ & $\begin{array}{r}1-0 \\
\text { TRANSPORT } \\
\text { THEORY } \\
\text { (N/CM } 2 / S \text { ) }\end{array}$ & $\begin{array}{r}2-0 \\
\text { DIFFUSION } \\
\text { THEORY } \\
\text { (N/CM2/S) }\end{array}$ \\
\hline $\begin{array}{r}1 \\
2 \\
3 \\
4 \\
5 \\
6 \\
7 \\
8 \\
9 \\
10 \\
11 \\
12 \\
13 \\
14 \\
15 \\
16 \\
17 \\
18 \\
19 \\
20 \\
21 \\
22 \\
23 \\
24 \\
25 \\
26 \\
27 \\
28 \\
29 \\
30 \\
31 \\
32 \\
33 \\
34 \\
35 \\
36 \\
37 \\
38 \\
39 \\
40 \\
41 \\
42 \\
43 \\
44 \\
45 \\
46 \\
47 \\
48 \\
49 \\
50\end{array}$ & $\begin{array}{l}1.9971 E+07 \\
1.0000 E+07 \\
6.0653 E+06 \\
3.6788 E+06 \\
2.2313 E+06 \\
1.3534 E+06 \\
8.2085 E+05 \\
4.9787 E+05 \\
3.8774 E+05 \\
3.0197 E+05 \\
2.3518 E+05 \\
1.8316 E+05 \\
1.4264 E+05 \\
1.1109 E+05 \\
8.6517 E+04 \\
6.7379 E+04 \\
5.2475 E+04 \\
4.0868 E+04 \\
3.1828 E+04 \\
2.4788 E+04 \\
1.9305 E+04 \\
1.5034 E+04 \\
1.1709 E+04 \\
9.1188 E+03 \\
7.1017 E+03 \\
5.5308 E+03 \\
4.3074 E+03 \\
3.3546 E+03 \\
2.6126 E+03 \\
2.0347 E+03 \\
1.5846 E+03 \\
1.2341 E+03 \\
9.6112 E+02 \\
7.4852 E+02 \\
5.8295 E+02 \\
4.5400 E+02 \\
3.5358 E+02 \\
2.7536 E+02 \\
1.6702 E+02 \\
1.0130 E+02 \\
6.1442 E+01 \\
3.7267 E+01 \\
2.2603 E+01 \\
1.3710 E+01 \\
8.3153 E+00 \\
5.0435 E+00 \\
3.0590 E+00 \\
1.8554 E+00 \\
1.1254 E+00 \\
6.8256 E-01 \\
1.0000 E-05\end{array}$ & $\begin{array}{l}9.3732 E-03 \\
9.6387 E-02 \\
3.4554 E-01 \\
6.7357 E-01 \\
1.0108 E+00 \\
2.5851 E+00 \\
9.5054 E+00 \\
6.4394 E+00 \\
7.9028 E+00 \\
8.1604 E+00 \\
7.5986 E+00 \\
7.1533 E+00 \\
6.9823 E+00 \\
6.5672 E+00 \\
7.0448 E+00 \\
6.3456 E+00 \\
6.0901 E+00 \\
4.2310 E+00 \\
2.5873 E+00 \\
3.9269 E+00 \\
1.8456 E+00 \\
1.2127 E+00 \\
7.0491 E-01 \\
3.1163 E-01 \\
2.3953 E-01 \\
1.4538 E-01 \\
1.1999 E-01 \\
6.5930 E-02 \\
4.1011 E-02 \\
3.0918 E-02 \\
1.3781 E-02 \\
5.3810 E-03 \\
3.5178 E-03 \\
1.5950 E-03 \\
9.2453 E-04 \\
5.0590 E-04 \\
3.3798 E-04 \\
3.8117 E-04 \\
5.4537 E-05 \\
1.0620 E-05 \\
1.7485 E-06 \\
1.9686 E-00 \\
1.1065 E-00 \\
1.7112 E-09 \\
3.3120 E-11 \\
2.2387 E-11 \\
4.8682 E-11 \\
2.8071 E-11 \\
5.8584 E-12 \\
3.5988 E-13 \\
1.0000 E+02\end{array}$ & $\begin{array}{l}1.0297 \mathrm{E}-02 \\
1.0108 \mathrm{E}-01 \\
3.5753 \mathrm{E}-01 \\
6.9058 \mathrm{E}-01 \\
1.0472 \mathrm{E}+00 \\
2.7044 \mathrm{E}+00 \\
9.5069 \mathrm{E}+00 \\
6.4265 \mathrm{E}+00 \\
7.8547 \mathrm{E}+00 \\
8.1227 \mathrm{E}+00 \\
7.5923 \mathrm{E}+00 \\
7.1712 \mathrm{E}+00 \\
7.0090 \mathrm{E}+00 \\
6.6360 \mathrm{E}+00 \\
7.1106 \mathrm{E}+00 \\
6.4487 \mathrm{E}+00 \\
6.1076 \mathrm{E}+00 \\
4.1718 \mathrm{E}+00 \\
2.5198 \mathrm{E}+00 \\
3.7962 \mathrm{E}+00 \\
1.8001 \mathrm{E}+00 \\
1.1850 \mathrm{E}+00 \\
6.8842 \mathrm{E}-01 \\
3.0810 \mathrm{E}-01 \\
2.3652 \mathrm{E}-01 \\
1.4354 \mathrm{E}-01 \\
1.1611 \mathrm{E}-01 \\
6.4444 \mathrm{E}-02 \\
3.4267 \mathrm{E}-02 \\
1.8977 \mathrm{E}-02 \\
9.9254 \mathrm{E}-03 \\
3.8697 \mathrm{E}-03 \\
2.4898 \mathrm{E}-03 \\
1.1819 \mathrm{E}-03 \\
7.4379 \mathrm{E}-04 \\
4.5010 \mathrm{E}-04 \\
2.9992 \mathrm{E}-04 \\
2.2415 \mathrm{E}-04 \\
3.8853 \mathrm{E}-05 \\
7.1630 \mathrm{E}-00 \\
1.3512 \mathrm{E}-06 \\
1.5412 \mathrm{E}-07 \\
8.6634 \mathrm{E}-09 \\
1.5687 \mathrm{E}-09 \\
2.9121 \mathrm{E}-11 \\
3.1721 \mathrm{E}-11 \\
7.5976 \mathrm{E}-11 \\
4.2598 \mathrm{E}-11 \\
8.7487 \mathrm{E}-12 \\
5.3623 \mathrm{E}-13 \\
1.0000 \mathrm{E}+02\end{array}$ & $\begin{array}{l}9.0423 E-03 \\
9.2660 E-02 \\
3.3382 E-01 \\
6.5825 E-01 \\
1.0053 E+00 \\
2.6569 E+00 \\
9.5966 E+00 \\
6.5539 E+00 \\
7.9915 E+00 \\
8.2410 E+00 \\
7.7043 E+00 \\
7.2439 E+00 \\
7.0354 E+00 \\
6.6434 E+00 \\
7.0698 E+00 \\
6.3304 E+00 \\
6.0004 E+00 \\
4.1055 E+00 \\
2.5589 E+00 \\
3.6820 E+00 \\
1.7383 E+00 \\
1.1383 E+00 \\
6.6057 E-01 \\
3.0036 E-01 \\
2.3006 E-01 \\
1.4003 E-01 \\
1.1681 E-01 \\
6.4375 E-02 \\
3.9409 E-02 \\
3.0040 E-02 \\
1.4478 E-02 \\
5.8316 E-03 \\
3.8943 E-03 \\
1.8544 E-03 \\
1.1056 E-03 \\
6.2798 E-04 \\
4.1817 E-04 \\
4.3878 E-04 \\
6.6886 E-05 \\
1.2340 E-05 \\
2.1659 E-06 \\
2.3732 E-07 \\
1.3418 E-08 \\
2.2290 E-09 \\
4.4313 E-11 \\
3.9509 E-11 \\
8.4244 E-11 \\
4.4668 E-11 \\
8.9229 E-12 \\
5.6147 E-13 \\
1.0000 E+02\end{array}$ \\
\hline
\end{tabular}


TABLE B-VI

VERA-11A CENTRAL ADJOINT SPECTRA

\begin{tabular}{|c|c|c|c|c|}
\hline GROUP & $\begin{array}{r}\text { ENERGY } \\
\text { BOUNDARY } \\
\text { (EV) }\end{array}$ & $\begin{array}{r}1-0 \\
\text { DIFFUSION } \\
\text { THEORY } \\
\text { (RHO/N/S) }\end{array}$ & $\begin{array}{r}1-D \\
\text { TRANSPORT } \\
\text { THEORY } \\
\text { (RHO/N/S) }\end{array}$ & $\begin{array}{r}2-0 \\
\text { DIFFUSION } \\
\text { THEORY } \\
\text { (RHO/N/S }\end{array}$ \\
\hline $\begin{array}{l}1 \\
2 \\
3 \\
4 \\
5 \\
5 \\
7 \\
8 \\
9 \\
10 \\
11 \\
12 \\
13 \\
14 \\
15 \\
16 \\
17 \\
18 \\
19 \\
20 \\
21 \\
22 \\
23 \\
24 \\
25 \\
26 \\
27 \\
28 \\
29 \\
30 \\
31 \\
32 \\
33 \\
34 \\
35 \\
36 \\
37 \\
38 \\
39 \\
40 \\
41 \\
42 \\
43 \\
44 \\
45 \\
46 \\
47 \\
48 \\
49 \\
50\end{array}$ & $\begin{array}{l}1.9971 E+07 \\
1.0000 E+07 \\
6.0653 E+06 \\
3.6788 E+06 \\
2.2313 E+06 \\
1.3534 E+06 \\
8.2085 E+05 \\
4.9787 E+05 \\
3.8774 E+05 \\
3.0197 E+05 \\
2.3518 E+05 \\
1.8316 E+05 \\
1.4264 E+05 \\
1.1109 E+05 \\
8.6517 E+04 \\
6.7379 E+04 \\
5.2475 E+04 \\
4.0868 E+04 \\
3.1828 E+04 \\
2.4788 E+04 \\
1.9305 E+04 \\
1.5034 E+04 \\
1.1709 E+04 \\
9.1188 E+03 \\
7.1017 E+03 \\
5.5308 E+03 \\
4.3074 E+03 \\
3.3546 E+03 \\
2.6126 E+03 \\
2.0347 E+03 \\
1.5846 E+03 \\
1.2341 E+03 \\
9.6112 E+02 \\
7.4852 E+02 \\
5.8295 E+02 \\
4.5100 E+02 \\
3.5358 E+02 \\
2.7536 E+02 \\
1.6702 E+02 \\
1.0130 E+02 \\
6.1442 E+01 \\
3.7267 E+01 \\
2.2603 E+01 \\
1.3710 E+01 \\
8.3153 E+00 \\
5.0435 E+00 \\
3.0590 E+00 \\
1.8554 E+00 \\
1.1254 E+00 \\
6.8256 E-01 \\
1.0000 E-05\end{array}$ & $\begin{array}{l}1.2679 E+00 \\
1.0692 E+00 \\
9.9075 E-01 \\
1.0230 E+00 \\
9.7985 E-01 \\
9.5466 E-01 \\
9.8598 E-01 \\
1.0206 E+00 \\
1.0343 E+00 \\
1.0537 E+00 \\
1.0743 E+00 \\
1.0895 E+00 \\
1.1009 E+00 \\
1.1101 E+00 \\
1.1122 E+00 \\
1.1142 E+00 \\
1.1180 E+00 \\
1.1283 E+00 \\
1.1314 E+00 \\
1.1146 E+00 \\
1.1284 E+00 \\
1.1390 E+00 \\
1.1569 E+00 \\
1.1739 E+00 \\
1.1732 E+00 \\
1.1989 E+00 \\
1.2338 E+00 \\
1.2591 E+00 \\
1.2658 E+00 \\
1.3323 E+00 \\
1.4317 E+00 \\
1.4314 E+00 \\
1.4354 E+00 \\
1.4508 E+00 \\
1.5973 E+00 \\
1.5519 E+00 \\
1.5337 E+00 \\
1.5873 E+00 \\
1.7114 E+00 \\
2.0273 E+00 \\
1.5475 E+00 \\
1.5727 E+00 \\
1.3903 E+00 \\
1.9434 E+00 \\
1.9500 E+00 \\
2.1317 E+00 \\
1.9047 E+00 \\
1.1728 E+00 \\
8.3568 E-01 \\
2.0431 E+00 \\
6.6285 E+01\end{array}$ & $\begin{array}{l}1.2791 E+00 \\
1.0938 E+00 \\
1.0096 E+00 \\
1.0277 E+00 \\
9.8834 E-01 \\
9.5605 E-01 \\
9.6937 E-01 \\
9.9187 E-01 \\
1.0021 E+00 \\
1.0177 E+00 \\
1.0358 E+00 \\
1.0498 E+00 \\
1.0608 E+00 \\
1.0696 E+00 \\
1.0728 E+00 \\
1.0748 E+00 \\
1.0793 E+00 \\
1.0900 E+00 \\
1.0950 E+00 \\
1.0766 E+00 \\
1.0894 E+00 \\
1.0990 E+00 \\
1.1159 E+00 \\
1.1319 E+00 \\
1.1304 E+00 \\
1.1559 E+00 \\
1.1876 E+00 \\
1.2123 E+00 \\
1.2192 E+00 \\
1.2760 E+00 \\
1.3685 E+00 \\
1.3690 E+00 \\
1.3727 E+00 \\
1.3886 E+00 \\
1.5296 E+00 \\
1.4826 E+00 \\
1.4664 E+00 \\
1.5165 E+00 \\
1.6354 E+00 \\
1.9372 E+00 \\
1.4816 E+00 \\
1.5012 E+00 \\
1.8091 E+00 \\
1.8580 E+00 \\
1.8587 E+00 \\
2.0207 E+00 \\
1.8192 E+00 \\
1.1185 E+00 \\
7.9700 E-01 \\
1.9465 E+00 \\
6.3926 E+01\end{array}$ & $\begin{array}{l}1.2711 E+00 \\
1.0714 E+00 \\
9.9216 E-01 \\
1.0238 E+00 \\
9.8030 E-01 \\
9.5403 E-01 \\
9.8479 E-01 \\
1.0190 E+00 \\
1.0326 E+00 \\
1.0517 E+00 \\
1.0720 E+00 \\
1.0870 E+00 \\
1.0981 E+00 \\
1.1069 E+00 \\
1.1087 E+00 \\
1.1102 E+00 \\
1.1136 E+00 \\
1.1236 E+00 \\
1.1266 E+00 \\
1.1097 E+00 \\
1.1233 E+00 \\
1.1337 E+00 \\
1.1513 E+00 \\
1.1682 E+00 \\
1.1673 E+00 \\
1.1926 E+00 \\
1.2272 E+00 \\
1.2523 E+00 \\
1.2592 E+00 \\
1.3256 E+00 \\
1.4249 E+00 \\
1.4248 E+00 \\
1.4290 E+00 \\
1.4445 E+00 \\
1.5905 E+00 \\
1.5452 E+00 \\
1.5273 E+00 \\
1.5809 E+00 \\
1.7048 E+00 \\
2.0196 E+00 \\
1.5414 E+00 \\
1.5657 E+00 \\
1.8832 E+00 \\
1.9359 E+00 \\
1.9420 E+00 \\
2.1227 E+00 \\
1.8975 E+00 \\
1.1684 E+00 \\
8.3246 E-01 \\
2.0352 E+00 \\
6.6054 E+01\end{array}$ \\
\hline
\end{tabular}


TABLE B-VII

ZPR-3-48 CENTRAL FLUX SPECTRA

\begin{tabular}{|c|c|c|c|c|}
\hline GROUP & $\begin{array}{r}\text { ENERGY } \\
\text { BOUNDARY } \\
\text { (EV) }\end{array}$ & $\begin{array}{r}1-0 \\
\text { DIFFUSION } \\
\text { THEORY } \\
\text { (N/CM2/S) }\end{array}$ & $\begin{array}{r}1-0 \\
\text { TRANSPORT } \\
\text { THEORY } \\
(\mathrm{N} / \mathrm{CM} 2 / \mathrm{S})\end{array}$ & $\begin{array}{r}2-0 \\
\text { DIFFUSION } \\
\text { THEORY } \\
\text { (N/CM2/S) }\end{array}$ \\
\hline $\begin{array}{r}1 \\
2 \\
3 \\
4 \\
5 \\
6 \\
7 \\
8 \\
9 \\
10 \\
11 \\
12 \\
13 \\
14 \\
15 \\
16 \\
17 \\
18 \\
19 \\
20 \\
21 \\
22 \\
23 \\
24 \\
25 \\
26 \\
27 \\
28 \\
29 \\
30 \\
31 \\
32 \\
33 \\
34 \\
35 \\
36 \\
37 \\
38 \\
39 \\
40 \\
41 \\
42 \\
43 \\
44 \\
45 \\
46 \\
47 \\
48 \\
49 \\
50\end{array}$ & $\begin{array}{l}1.9971 E+07 \\
1.0000 E+07 \\
6.0653 E+06 \\
3.6788 E+06 \\
2.2313 E+06 \\
1.3534 E+06 \\
8.2085 E+05 \\
4.9787 E+05 \\
3.8774 E+05 \\
3.0197 E+05 \\
2.3518 E+05 \\
1.8316 E+05 \\
1.4264 E+05 \\
1.1109 E+05 \\
8.6517 E+04 \\
6.7379 E+04 \\
5.2475 E+04 \\
4.0868 E+04 \\
3.1828 E+04 \\
2.4788 E+04 \\
1.9305 E+04 \\
1.5034 E+04 \\
1.1709 E+04 \\
9.1188 E+03 \\
7.1017 E+03 \\
5.5308 E+03 \\
4.3074 E+03 \\
3.3546 E+03 \\
2.6126 E+03 \\
2.0347 E+03 \\
1.5846 E+03 \\
1.2341 E+03 \\
9.6112 E+02 \\
7.4852 E+02 \\
5.8295 E+02 \\
4.5400 E+02 \\
3.5358 E+02 \\
2.7536 E+02 \\
1.6702 E+02 \\
1.0130 E+02 \\
6.1442 E+01 \\
3.7267 E+01 \\
2.2603 E+01 \\
1.3710 E+01 \\
8.3153 E+00 \\
5.0435 E+00 \\
3.0590 E+00 \\
1.8554 E+00 \\
1.1254 E+00 \\
6.8256 E-01 \\
1.0000 E-05\end{array}$ & $\begin{array}{l}3.5278 E-02 \\
3.8344 E-01 \\
1.7067 E+00 \\
3.9565 E+00 \\
6.0906 E+00 \\
7.7415 E+00 \\
1.0247 E+01 \\
5.6878 E+00 \\
5.8219 E+00 \\
5.5697 E+00 \\
5.2095 E+00 \\
5.0695 E+00 \\
4.7649 E+00 \\
4.3732 E+00 \\
4.1214 E+00 \\
3.6774 E+00 \\
3.4879 E+00 \\
2.9293 E+00 \\
2.6131 E+00 \\
2.7887 E+00 \\
2.3106 E+00 \\
2.0540 E+00 \\
1.6935 E+00 \\
1.3098 E+00 \\
1.1819 E+00 \\
9.6315 E-01 \\
6.1041 E-01 \\
2.0884 E-01 \\
5.2292 E-01 \\
6.9762 E-01 \\
6.1702 E-01 \\
4.5075 E-01 \\
3.4713 E-01 \\
2.6062 E-01 \\
1.7210 E-01 \\
1.2347 E-01 \\
7.7597 E-02 \\
8.3594 E-02 \\
2.9701 E-02 \\
7.5046 E-03 \\
1.7859 E-03 \\
7.9496 E-04 \\
1.2650 E-04 \\
2.9537 E-05 \\
4.2358 E-06 \\
2.3337 E-06 \\
1.1955 E-06 \\
2.4879 E-07 \\
1.6489 E-08 \\
5.0378 E-10 \\
1.0090 E+02\end{array}$ & $\begin{array}{l}3.5256 E-02 \\
3.8206 E-01 \\
1.6970 E+00 \\
3.9312 E+00 \\
6.0582 E+00 \\
7.7115 E+00 \\
1.0225 E+01 \\
5.6783 E+00 \\
5.8168 E+00 \\
5.5672 E+00 \\
5.2094 E+00 \\
5.0705 E+00 \\
4.7680 E+00 \\
4.3759 E+00 \\
4.1276 E+00 \\
3.6832 E+00 \\
3.4937 E+00 \\
2.9330 E+00 \\
2.6265 E+00 \\
2.8025 E+00 \\
2.3228 E+00 \\
2.0649 E+00 \\
1.7026 E+00 \\
1.3170 E+00 \\
1.1885 E+00 \\
9.6847 E-01 \\
6.1405 E-01 \\
2.1001 E-01 \\
5.2560 E-01 \\
7.0247 E-01 \\
6.2142 E-01 \\
4.5423 E-01 \\
3.5005 E-01 \\
2.6302 E-01 \\
1.7380 E-01 \\
1.2471 E-01 \\
7.8421 E-02 \\
8.4697 E-02 \\
3.0148 E-02 \\
7.6325 E-03 \\
1.8189 E-03 \\
8.1196 E-04 \\
1.2948 E-04 \\
3.0281 E-05 \\
4.3516 E-06 \\
2.3989 E-06 \\
1.2299 E-06 \\
2.5621 E-07 \\
1.6997 E-08 \\
5.1940 E-10 \\
1.0000 E+02\end{array}$ & $\begin{array}{l}3.5322 E-02 \\
3.8403 E-01 \\
1.7093 E+00 \\
3.9617 E+00 \\
6.0957 E+00 \\
7.7443 E+00 \\
1.0247 E+01 \\
5.6871 E+00 \\
5.8205 E+00 \\
5.5680 E+00 \\
5.2079 E+00 \\
5.0680 E+00 \\
4.7635 E+00 \\
4.3720 E+00 \\
4.1204 E+00 \\
3.6766 E+00 \\
3.4874 E+00 \\
2.9290 E+00 \\
2.6130 E+00 \\
2.7887 E+00 \\
2.3107 E+00 \\
2.0541 E+00 \\
1.6935 E+00 \\
1.3097 E+00 \\
1.1817 E+00 \\
9.6296 E-01 \\
6.1025 E-01 \\
2.0878 E-01 \\
5.2275 E-01 \\
6.9727 E-01 \\
6.1654 E-01 \\
4.5027 E-01 \\
3.4662 E-01 \\
2.6012 E-01 \\
1.7170 E-01 \\
1.2313 E-01 \\
7.7355 E-02 \\
8.3275 E-02 \\
2.9569 E-02 \\
7.4674 E-03 \\
1.7763 E-03 \\
7.8986 E-04 \\
1.2564 E-04 \\
2.9320 E-05 \\
4.2034 E-06 \\
2.3125 E-06 \\
1.1830 E-06 \\
2.4605 E-07 \\
1.6305 E-08 \\
4.9816 E-10 \\
1.0000 E+02\end{array}$ \\
\hline
\end{tabular}


TABLE B-VIII

ZPR-3-48 EDGE FLUX SPECTRA

\begin{tabular}{|c|c|c|c|c|}
\hline GROUP & $\begin{array}{r}\text { ENERGY } \\
\text { BOUNDRRY } \\
\text { (EV) }\end{array}$ & $\begin{array}{r}1-0 \\
\text { OIFFUSION } \\
\text { THEORY } \\
\text { (N/CM2/S) }\end{array}$ & $\begin{array}{r}1-D \\
\text { TRANSPORT } \\
\text { THEORY } \\
\text { [N/CM2/S }\end{array}$ & $\begin{array}{r}2-D \\
\text { OIFFUSION } \\
\text { THEORY } \\
\text { (N/CM2/S) }\end{array}$ \\
\hline $\begin{array}{l}1 \\
2 \\
3 \\
4 \\
5 \\
6 \\
7 \\
8 \\
9 \\
10 \\
11 \\
12 \\
13 \\
14 \\
15 \\
16 \\
17 \\
18 \\
19 \\
20 \\
21 \\
22 \\
23 \\
24 \\
25 \\
26 \\
27 \\
28 \\
29 \\
30 \\
31 \\
32 \\
33 \\
34 \\
35 \\
36 \\
37 \\
38 \\
39 \\
40 \\
41 \\
42 \\
43 \\
44 \\
45 \\
46 \\
47 \\
48 \\
49 \\
50\end{array}$ & $\begin{array}{l}1.9971 E+07 \\
1.0000 E+07 \\
6.0653 E+06 \\
3.6788 E+06 \\
2.2313 E+06 \\
1.3534 E+06 \\
8.2085 E+05 \\
4.9787 E+05 \\
3.8774 E+05 \\
3.0197 E+05 \\
2.3518 E+05 \\
1.8316 E+05 \\
1.4264 E+05 \\
1.1109 E+05 \\
8.6517 E+04 \\
6.7379 E+04 \\
5.2475 E+04 \\
4.0868 E+04 \\
3.1828 E+04 \\
2.4788 E+04 \\
1.9305 E+04 \\
1.5034 E+04 \\
1.1709 E+04 \\
9.1188 E+03 \\
7.1017 E+03 \\
5.5308 E+03 \\
4.3074 E+03 \\
3.3546 E+03 \\
2.6126 E+03 \\
2.0347 E+03 \\
1.5846 E+03 \\
1.2341 E+03 \\
9.6112 E+02 \\
7.4852 E+02 \\
5.8295 E+02 \\
4.5400 E+02 \\
3.5358 E+02 \\
2.7536 E+02 \\
1.6702 E+02 \\
1.0130 E+02 \\
6.1442 E+01 \\
3.7267 E+01 \\
2.2603 E+01 \\
1.3710 E+01 \\
8.3153 E+00 \\
5.0435 E+00 \\
3.0590 E+00 \\
1.8554 E+00 \\
1.1254 E+00 \\
6.8256 E-01 \\
1.0000 E-05\end{array}$ & $\begin{array}{l}5.5165 E-03 \\
5.3197 E-02 \\
1.8807 E-01 \\
3.7096 E-01 \\
6.0099 E-01 \\
1.9550 E+00 \\
8.4677 E+00 \\
6.0130 E+00 \\
7.4163 E+00 \\
7.8147 E+00 \\
7.4813 E+00 \\
7.1322 E+00 \\
7.0125 E+00 \\
6.9178 E+00 \\
7.3443 E+00 \\
6.7153 E+00 \\
6.5554 E+00 \\
4.7762 E+00 \\
3.0698 E+00 \\
3.9314 E+00 \\
2.0890 E+00 \\
1.4091 E+00 \\
8.7510 E-01 \\
4.3156 E-01 \\
3.3471 E-01 \\
2.2355 E-01 \\
1.9626 E-01 \\
8.6375 E-02 \\
5.9954 E-02 \\
1.6050 E-01 \\
1.0101 E-01 \\
4.9211 E-02 \\
5.3171 E-02 \\
3.1035 E-02 \\
2.4107 E-02 \\
1.4704 E-02 \\
9.3990 E-03 \\
2.4924 E-02 \\
3.7435 E-03 \\
8.3825 E-04 \\
2.3118 E-04 \\
2.9905 E-05 \\
1.7134 E-06 \\
9.3499 E-07 \\
1.6120 E-08 \\
2.3815 E-08 \\
5.9401 E-08 \\
3.5951 E-08 \\
8.7187 E-09 \\
7.9613 E-10 \\
1.0000 E+02\end{array}$ & $\begin{array}{l}7.3405 E-03 \\
6.7967 E-02 \\
2.3695 E-01 \\
4.5773 E-01 \\
7.3442 E-01 \\
2.1924 E+00 \\
8.5640 E+00 \\
6.0259 E+00 \\
7.3925 E+00 \\
7.7887 E+00 \\
7.4724 E+00 \\
7.1411 E+00 \\
7.0303 E+00 \\
6.9607 E+00 \\
7.3901 E+00 \\
6.7894 E+00 \\
6.5463 E+00 \\
4.7151 E+00 \\
2.9985 E+00 \\
3.7978 E+00 \\
2.0176 E+00 \\
1.3556 E+00 \\
8.3593 E-01 \\
4.1468 E-01 \\
3.1822 E-01 \\
2.1083 E-01 \\
1.7931 E-01 \\
7.9437 E-02 \\
4.7368 E-02 \\
5.4301 E-02 \\
4.8965 E-02 \\
2.6289 E-02 \\
3.0563 E-02 \\
1.9756 E-02 \\
1.7877 E-02 \\
1.2366 E-02 \\
7.9427 E-03 \\
1.0531 E-02 \\
2.1283 E-03 \\
4.5884 E-04 \\
1.5960 E-04 \\
2.1734 E-05 \\
1.2494 E-06 \\
8.7583 E-07 \\
1.4766 E-08 \\
2.6948 E-08 \\
6.6632 E-08 \\
3.9799 E-08 \\
9.5734 E-09 \\
8.7085 E-10 \\
1.0000 E+02\end{array}$ & $\begin{array}{l}4.6785 E-03 \\
4.5479 E-02 \\
1.6114 E-01 \\
3.1621 E-01 \\
5.0946 E-01 \\
1.7281 E+00 \\
8.0695 E+00 \\
5.7992 E+00 \\
7.2831 E+00 \\
7.7853 E+00 \\
7.5589 E+00 \\
7.3673 E+00 \\
7.3816 E+00 \\
7.4424 E+00 \\
7.8697 E+00 \\
7.2033 E+00 \\
6.7084 E+00 \\
4.8738 E+00 \\
3.5728 E+00 \\
3.4730 E+00 \\
1.9505 E+00 \\
1.2526 E+00 \\
7.1417 E-01 \\
3.6367 E-01 \\
2.4214 E-01 \\
1.4505 E-01 \\
1.0229 E-01 \\
2.4669 E-02 \\
1.8273 E-02 \\
1.2262 E-02 \\
8.4306 E-03 \\
4.1781 E-03 \\
3.0725 E-03 \\
1.7710 E-03 \\
1.3245 E-03 \\
8.5170 E-04 \\
4.5984 E-04 \\
5.6574 E-04 \\
8.8100 E-05 \\
1.3922 E-05 \\
5.8707 E-06 \\
7.5449 E-07 \\
2.2470 E-08 \\
1.8068 E-07 \\
1.7721 E-09 \\
1.0486 E-08 \\
3.9224 E-08 \\
2.5584 E-08 \\
6.1415 E-09 \\
5.2919 E-10 \\
1.0000 E+02\end{array}$ \\
\hline
\end{tabular}


:の-ー -

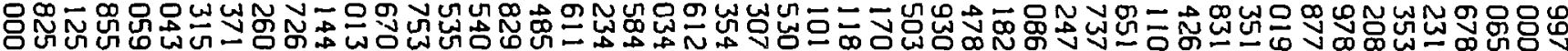

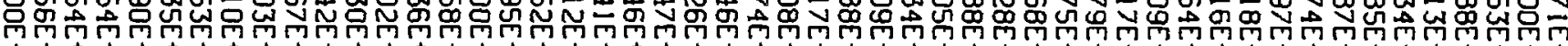

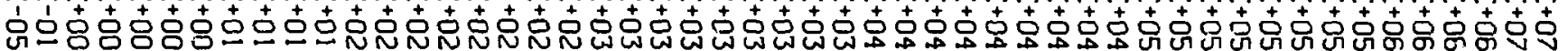

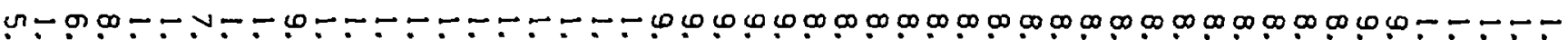

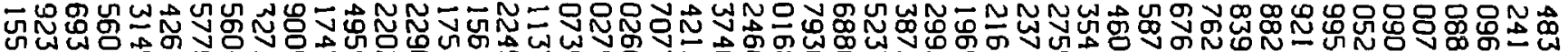

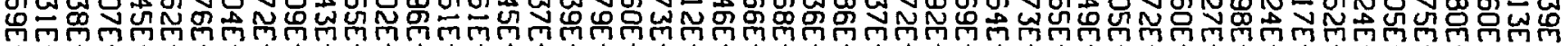

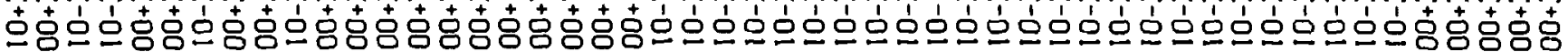

תー ח

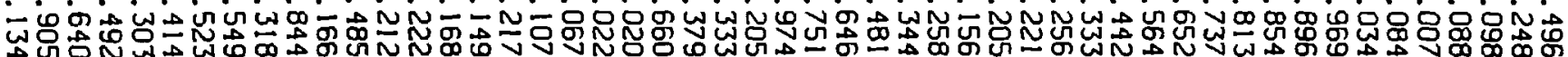

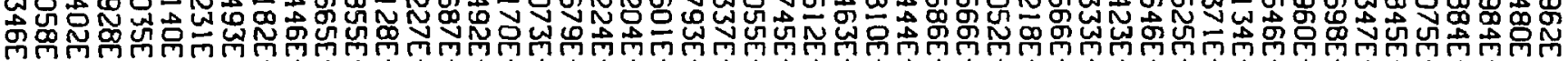

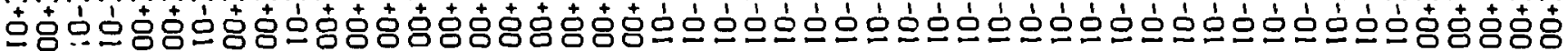

ฯ -

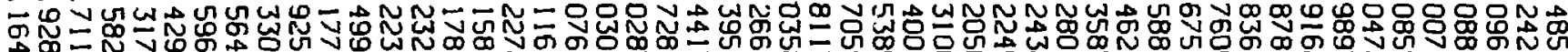

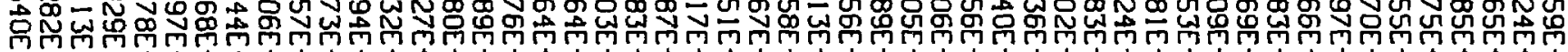

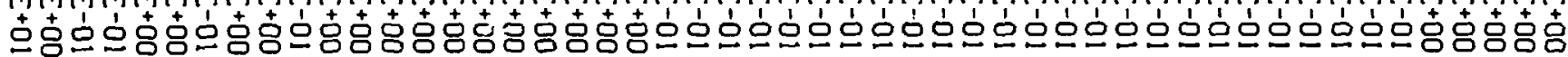

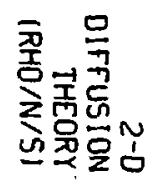




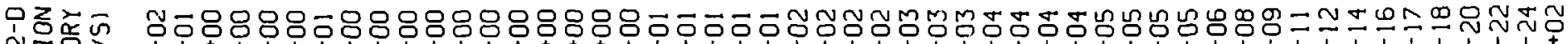

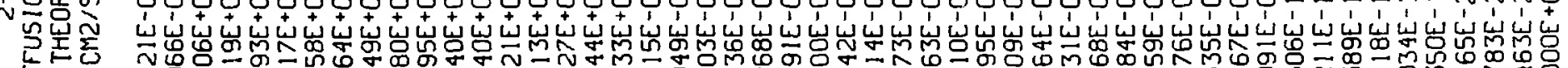

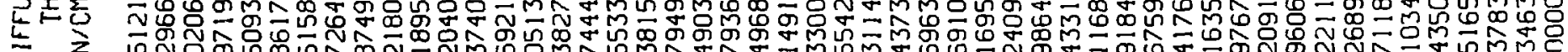

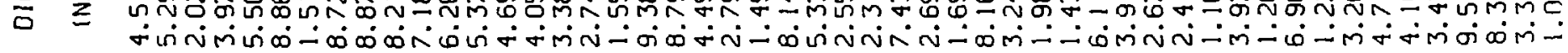

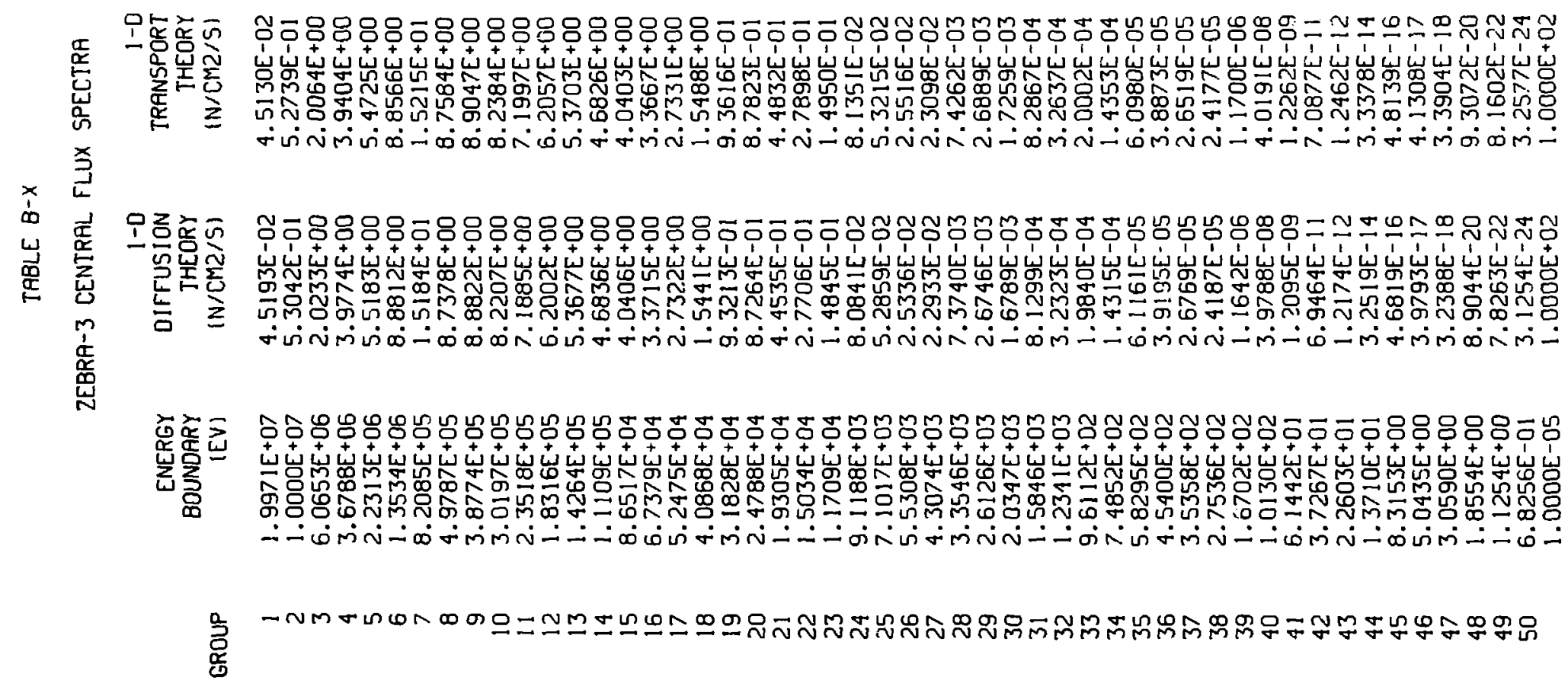




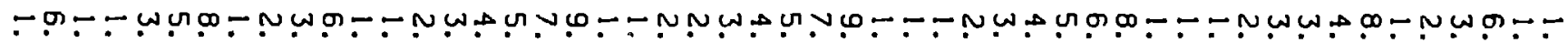

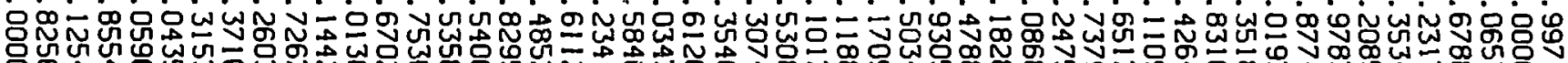

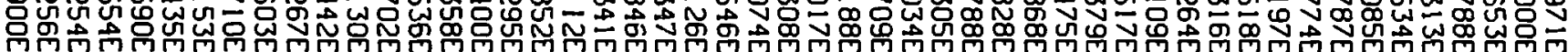

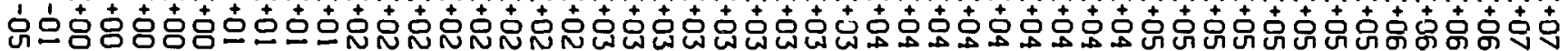

م

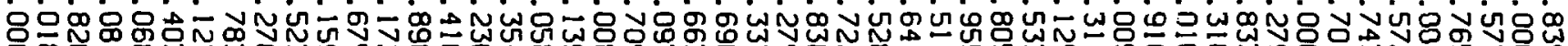

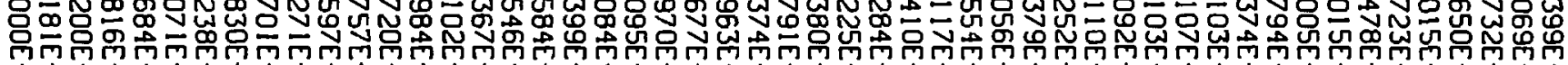

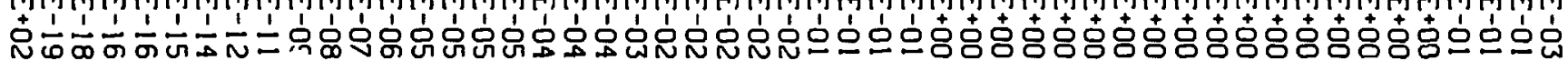

-

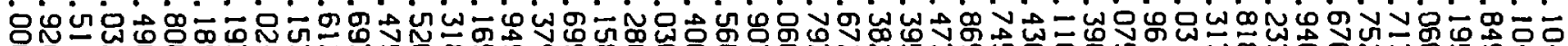

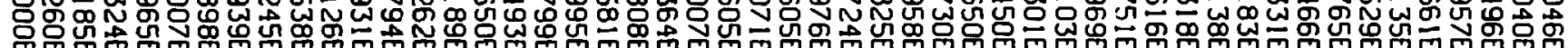

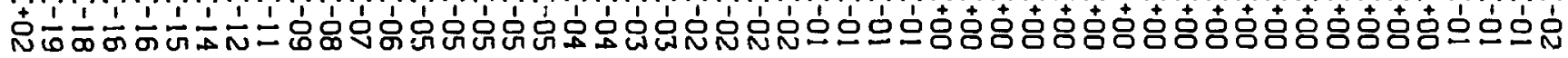

-

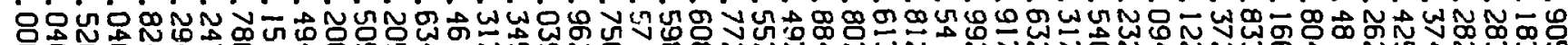

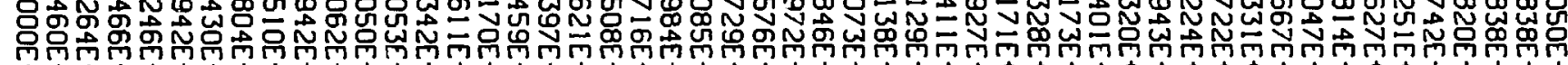

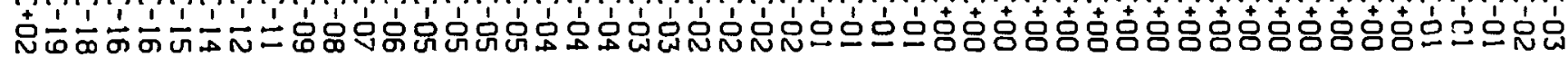

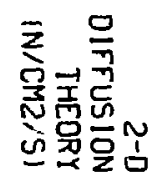


TABLE $B-\times 11$

ZEBRA-3 CENTRAL AOJOINT SPECTRA

\begin{tabular}{|c|c|c|c|c|}
\hline GROUP & $\begin{array}{r}\text { ENERGY } \\
\text { BOUNDRRY } \\
\text { (EV) }\end{array}$ & $\begin{array}{r}1-D \\
\text { OIFFUSION } \\
\text { THEORY } \\
\text { (RHO/N/S) }\end{array}$ & $\begin{array}{r}1-D \\
\text { TRANSPORT } \\
\text { THEORY } \\
\text { (RHO/N/S) }\end{array}$ & $\begin{array}{r}2-0 \\
\text { DIFFUSION } \\
\text { THEORY } \\
\text { (RHO/N/S) }\end{array}$ \\
\hline $\begin{array}{l}1 \\
2 \\
3 \\
4 \\
5 \\
6 \\
7 \\
8 \\
9 \\
10 \\
11 \\
12 \\
13 \\
14 \\
15 \\
16 \\
17 \\
18 \\
19 \\
20 \\
21 \\
22 \\
23 \\
24 \\
25 \\
26 \\
27 \\
28 \\
29 \\
30 \\
31 \\
32 \\
33 \\
34 \\
35 \\
36 \\
37 \\
38 \\
39 \\
40 \\
41 \\
42 \\
43 \\
44 \\
45 \\
46 \\
47 \\
48 \\
49 \\
50\end{array}$ & $\begin{array}{l}1.9971 E+07 \\
1.0000 E+07 \\
6.0653 E+06 \\
3.6788 E+06 \\
2.2313 E+06 \\
1.3534 E+06 \\
8.2085 E+05 \\
4.9787 E+05 \\
3.8774 E+05 \\
3.0197 E+05 \\
2.3518 E+05 \\
1.8316 E+05 \\
1.4264 E+05 \\
1.1109 E+05 \\
8.6517 E+04 \\
6.7379 E+04 \\
5.2475 E+04 \\
4.0868 E+04 \\
3.1828 E+04 \\
2.4788 E+04 \\
1.9305 E+04 \\
1.5034 E+04 \\
1.1709 E+04 \\
9.1188 E+03 \\
7.1017 E+03 \\
5.5308 E+03 \\
4.3074 E+03 \\
3.3546 E+03 \\
2.6126 E+03 \\
2.0347 E+03 \\
1.5846 E+03 \\
1.2341 E+03 \\
9.6112 E+02 \\
7.4852 E+02 \\
5.8295 E+02 \\
4.5400 E+02 \\
3.5358 E+02 \\
2.7536 E+02 \\
1.6702 E+02 \\
1.0130 E+02 \\
6.1442 E+01 \\
3.7267 E+01 \\
2.2603 E+01 \\
1.3710 E+01 \\
8.3153 E+00 \\
5.0435 E+00 \\
3.0590 E+00 \\
1.8554 E+00 \\
1.1254 E+00 \\
6.8256 E-01 \\
1.0000 E-05\end{array}$ & $\begin{array}{l}2.0870 E+00 \\
1.5745 E+00 \\
1.1709 E+00 \\
1.1444 E+00 \\
1.0279 E+00 \\
8.2032 E-01 \\
8.0447 E-01 \\
8.1758 E-01 \\
8.1357 E-01 \\
8.0140 E-01 \\
8.0020 E-01 \\
7.9191 E-01 \\
7.7958 E-01 \\
7.5723 E-01 \\
7.3096 E-01 \\
6.9167 E-01 \\
6.5888 E-01 \\
6.3915 E-01 \\
6.1895 E-01 \\
5.9460 E-01 \\
5.8208 E-01 \\
5.7702 E-01 \\
5.8239 E-01 \\
5.9605 E-01 \\
5.8629 E-01 \\
6.1488 E-01 \\
6.6328 E-01 \\
6.8451 E-01 \\
6.4813 E-01 \\
7.0768 E-01 \\
8.7970 E-01 \\
8.7290 E-01 \\
8.6212 E-01 \\
8.3492 E-01 \\
1.1697 E+00 \\
1.0822 E+00 \\
1.0743 E+00 \\
1.1152 E+00 \\
1.0918 E+00 \\
1.4936 E+00 \\
1.2744 E+00 \\
7.3253 E-01 \\
1.1776 E+00 \\
1.6258 E+00 \\
5.0187 E-01 \\
1.3158 E+00 \\
1.5980 E+00 \\
1.0101 E+00 \\
6.7933 E-01 \\
1.9027 E+00 \\
4.6668 E+01\end{array}$ & $\begin{array}{l}2.1017 E+00 \\
1.5793 E+00 \\
1.1730 E+00 \\
1.1447 E+00 \\
1.0273 E+00 \\
8.2155 E-01 \\
8.0495 E-01 \\
8.1424 E-01 \\
8.0873 E-01 \\
8.0124 E-01 \\
7.9329 E-01 \\
7.8471 E-01 \\
7.7249 E-01 \\
7.5048 E-01 \\
7.2506 E-01 \\
6.8619 E-01 \\
6.5595 E-01 \\
6.3834 E-01 \\
6.1860 E-01 \\
5.9431 E-01 \\
5.8148 E-01 \\
5.7641 E-01 \\
5.8159 E-01 \\
5.9475 E-01 \\
5.8515 E-01 \\
6.1443 E-01 \\
6.6342 E-01 \\
6.8452 E-01 \\
6.5582 E-01 \\
7.2282 E-01 \\
8.8174 E-01 \\
8.7307 E-01 \\
8.6396 E-01 \\
8.3302 E-01 \\
1.1631 E+00 \\
1.0740 E+00 \\
1.0686 E+00 \\
1.1153 E+00 \\
1.0839 E+00 \\
1.4823 E+00 \\
1.2629 E+00 \\
7.2787 E-01 \\
1.1670 E+00 \\
1.6096 E+00 \\
4.9677 E-01 \\
1.2994 E+00 \\
1.5782 E+00 \\
9.9836 E-01 \\
6.7133 E-01 \\
1.8782 E+00 \\
4.6485 E+01\end{array}$ & $\begin{array}{l}2.0884 E+00 \\
1.5752 E+00 \\
1.1713 E+00 \\
1.1447 E+00 \\
1.0279 E+00 \\
8.2005 E-01 \\
8.0445 E-01 \\
8.1720 E-01 \\
8.1292 E-01 \\
8.0650 E-01 \\
7.9914 E-01 \\
7.9078 E-01 \\
7.7846 E-01 \\
7.5614 E-01 \\
7.2993 E-01 \\
6.9072 E-01 \\
6.5795 E-01 \\
6.3828 E-01 \\
6.1821 E-01 \\
5.9385 E-01 \\
5.8142 E-01 \\
5.7641 E-01 \\
5.8186 E-01 \\
5.9562 E-01 \\
5.8587 E-01 \\
6.1449 E-01 \\
6.6290 E-01 \\
6.8415 E-01 \\
6.4764 E-01 \\
7.0696 E-01 \\
8.7927 E-01 \\
8.7268 E-01 \\
8.6185 E-01 \\
8.3483 E-01 \\
1.1697 E+00 \\
1.0822 E+00 \\
1.0743 E+00 \\
1.1152 E+00 \\
1.0918 E+00 \\
1.4937 E+00 \\
1.2745 E+00 \\
7.3257 E-01 \\
1.1777 E+00 \\
1.6259 E+00 \\
5.0189 E-01 \\
1.3156 E+00 \\
1.5980 E+00 \\
1.0101 E+00 \\
6.7934 E-01 \\
1.9027 E+00 \\
4.6653 E+01\end{array}$ \\
\hline
\end{tabular}


TABLE B-XI11

GOOIVA CENTRAL FLUX SPECTRA

$\begin{array}{rrrr} & & 1-D & 1-D \\ & \text { ENERGY } & \text { DIFFUSION } & \text { TRANSPORT } \\ \text { GROUP } & \text { THEORY } & \text { THEORY } \\ & \text { IEVI } & \text { IN/CI12/SI } & \text { IN/CM2/SI } \\ 1 & 1.9971 E+C 7 & 1.1711 E-01 & 1.2692 E-01 \\ 2 & 1.0000 E+07 & 1.6462 E+00 & 1.7457 E+00 \\ 3 & 6.0653 E+06 & 6.7743 E+00 & 6.9544 E+00 \\ 4 & 3.6788 E+06 & 1.3780 E+01 & 1.3953 E+01 \\ 5 & 2.2313 E+06 & 1.7617 E+01 & 1.7767 E+01 \\ 6 & 1.3534 E+06 & 1.7414 E+01 & 1.7531 E+01 \\ 7 & 8.2085 E+05 & 1.5664 E+01 & 1.5620 E+01 \\ 8 & 4.9787 E+05 & 6.6334 E+00 & 6.5524 E+00 \\ 9 & 3.8774 E+05 & 5.4668 E+00 & 5.3651 E+00 \\ 10 & 3.0197 E+05 & 4.2277 E+00 & 4.1249 E+00 \\ 11 & 2.3518 E+05 & 3.1925 E+00 & 3.0980 E+00 \\ 12 & 1.8316 E+05 & 2.3612 E+00 & 2.2797 E+00 \\ 13 & 1.4264 E+05 & 1.7307 E+00 & 1.6637 E+00 \\ 14 & 1.1109 E+05 & 1.2364 E+00 & 1.1833 E+00 \\ 15 & 8.6517 E+04 & 8.1062 E-01 & 7.7228 E-01 \\ 16 & 6.7379 E+04 & 4.9120 E-01 & 4.6576 E-01 \\ 17 & 5.2475 E+04 & 3.4239 E-01 & 3.2409 E-01 \\ 18 & 4.0868 E+04 & 1.9677 E-01 & 1.8563 E-01 \\ 19 & 3.1828 E+04 & 1.1080 E-01 & 1.0430 E-01 \\ 20 & 2.4788 E+04 & 7.7582 E-02 & 7.5248 E-02 \\ 21 & 1.9305 E+04 & 4.1992 E-02 & 4.0694 E-02 \\ 22 & 1.5034 E+04 & 2.6176 E-02 & 2.5421 E-02 \\ 23 & 1.1709 E+04 & 1.5385 E-02 & 1.5000 E-02 \\ 24 & 9.1188 E+03 & 1.0270 E-02 & 1.0026 E-02 \\ 25 & 7.1017 E+03 & 5.7630 E-03 & 5.6440 E-03 \\ 26 & 5.5308 E+03 & 3.8476 E-03 & 3.7582 E-03 \\ 27 & 4.3074 E+03 & 2.2308 E-03 & 2.1881 E-03 \\ 28 & 3.3546 E+03 & 1.3946 E-03 & 1.3711 E-03 \\ 29 & 2.6126 E+03 & 8.8628 E-04 & 8.7298 E-04 \\ 30 & 2.0347 E+03 & 5.3952 E-04 & 5.3521 E-04 \\ 31 & 1.5846 E+03 & 3.3639 E-04 & 3.3500 E-04 \\ 32 & 1.2341 E+03 & 1.9766 E-04 & 1.9819 E-04 \\ 33 & 9.6112 E+02 & 1.2986 E-04 & 1.2994 E-04 \\ 34 & 7.4852 E+02 & 8.4714 E-05 & 8.5854 E-05 \\ 35 & 5.8295 E+02 & 5.4054 E-05 & 5.5464 E-05 \\ 35 & 4.5400 E+02 & 2.7752 E-05 & 2.8020 E-05 \\ 37 & 3.5358 E+02 & 2.6649 E-05 & 2.6914 E-05 \\ 38 & 2.7536 E+02 & 2.1717 E-05 & 2.1879 E-05 \\ 39 & 1.6702 E+02 & 2.3685 E-05 & 2.3992 E-05 \\ 40 & 1.0130 E+02 & 1.6122 E-07 & 1.6707 E-07 \\ 41 & 6.1442 E+01 & 4.5823 E-10 & 4.8574 E-10 \\ 42 & 3.7267 E+01 & 1.5480 E-12 & 1.6098 E-12 \\ 43 & 2.2603 E+01 & 5.5623 E-15 & 5.7997 E-15 \\ 44 & 1.3710 E+01 & 1.3178 E-17 & 1.3753 E-17 \\ 45 & 8.3153 E+00 & 5.0341 E-20 & 5.2639 E-20 \\ 46 & 5.0435 E+00 & 3.0036 E-22 & 3.1521 E-22 \\ 47 & 3.0590 E+00 & 1.6224 E-24 & 1.6994 E-24 \\ 48 & 1.8554 E+00 & 6.4256 E-27 & 6.7278 E-27 \\ 49 & 1.1254 E+00 & 1.0251 E-29 & 1.0732 E-29 \\ 50 & 6.8256 E-01 & 7.9702 E-33 & 8.3450 E-33 \\ & 1.0000 E-05 & 1.0000 E+02 & 1.0000 E+02\end{array}$


TAELE B-XIV

GODIVA EDGE FLUX SPECTRA

$\begin{array}{rrrr} & & 1-0 & 1-0 \\ & \text { ENERGY } & \text { DIFFUSION } & \text { TRANSPORT } \\ \text { GROUP } & \text { IHUUNDRRY } & \text { IHEORY } & \text { IHEORY } \\ & \text { IEV] } & \text { IN/CM2/SI } & \text { (N/CM2/S) } \\ 1 & 1.9971 E+07 & 1.6121 E-01 & 1.5073 E-01 \\ 2 & 1.0000 E+07 & 2.1371 E+00 & 2.0168 E+00 \\ 3 & 6.0653 E+06 & 7.9975 E+00 & 7.6669 E+00 \\ 4 & 3.6788 E+06 & 1.5383 E+01 & 1.4945 E+01 \\ 5 & 2.2313 E+06 & 1.9075 E+01 & 1.8724 E+01 \\ 6 & 1.3534 E+06 & 1.8309 E+01 & 1.8182 E+01 \\ 7 & 8.2085 E+05 & 1.5219 E+01 & 1.5448 E+01 \\ 8 & 4.9787 E+05 & 5.9731 E+00 & 6.1730 E+00 \\ 9 & 3.8774 E+05 & 4.6777 E+00 & 4.8842 E+00 \\ 10 & 3.0197 E+05 & 3.4505 E+00 & 3.6330 E+00 \\ 11 & 2.3518 E+05 & 2.4877 E+00 & 2.6400 E+00 \\ 12 & 1.8316 E+05 & 1.7453 E+00 & 1.8679 E+00 \\ 13 & 1.4264 E+05 & 1.2181 E+00 & 1.3130 E+00 \\ 14 & 1.1109 E+05 & 8.3005 E-01 & 9.0076 E-01 \\ 15 & 8.6517 E+04 & 5.2174 E-01 & 5.6902 E-01 \\ 16 & 6.7379 E+04 & 3.0532 E-01 & 3.3388 E-01 \\ 17 & 5.2475 E+04 & 2.0729 E-01 & 2.2744 E-01 \\ 18 & 4.0868 E+04 & 1.1724 E-01 & 1.2840 E-01 \\ 19 & 3.1828 E+04 & 6.5862 E-02 & 7.1674 E-02 \\ 20 & 2.4788 E+04 & 4.8514 E-02 & 5.1240 E-02 \\ 21 & 1.9305 E+04 & 2.6411 E-02 & 2.7682 E-02 \\ 22 & 1.5034 E+04 & 1.6588 E-02 & 1.7246 E-02 \\ 23 & 1.1709 E+04 & 9.9082 E-03 & 1.0164 E-02 \\ 24 & 9.1188 E+03 & 6.6165 E-03 & 6.7610 E-03 \\ 25 & 7.1017 E+03 & 3.8134 E-03 & 3.8358 E-03 \\ 26 & 5.5308 E+03 & 2.5356 E-03 & 2.5554 E-03 \\ 27 & 4.3074 E+03 & 1.5088 E-03 & 1.4939 E-03 \\ 28 & 3.3546 E+03 & 9.6336 E-04 & 9.4407 E-04 \\ 29 & 2.6126 E+03 & 6.1896 E-04 & 6.0034 E-04 \\ 30 & 2.0347 E+03 & 3.9111 E-04 & 3.6842 E-04 \\ 31 & 1.5846 E+03 & 2.4884 E-04 & 2.3171 E-04 \\ 32 & 1.2341 E+03 & 1.5173 E-04 & 1.3689 E-04 \\ 33 & 9.6112 E+02 & 9.9398 E-05 & 9.0056 E-05 \\ 34 & 7.4852 E+02 & 6.9314 E-05 & 5.9710 E-05 \\ 35 & 5.8295 E+02 & 4.6446 E-05 & 3.8702 E-05 \\ 36 & 4.5400 E+02 & 2.2462 E-05 & 1.9539 E-05 \\ 37 & 3.5358 E+02 & 2.1774 E-05 & 1.8793 E-05 \\ 38 & 2.7536 E+02 & 1.8146 E-05 & 1.5290 E-05 \\ 39 & 1.6702 E+02 & 1.9953 E-05 & 1.6787 E-05 \\ 40 & 1.0130 E+02 & 1.1424 E-07 & 9.8853 E-08 \\ 41 & 6.1442 E+01 & 2.6148 E-10 & 2.4778 E-10 \\ 42 & 3.7267 E+01 & 7.7899 E-13 & 7.5971 E-13 \\ 43 & 2.2603 E+01 & 2.6162 E-15 & 2.6197 E-15 \\ 44 & 1.3710 E+01 & 5.5448 E-18 & 5.7650 E-18 \\ 45 & 8.3153 E+00 & 2.1110 E-20 & 2.2373 E-20 \\ 46 & 5.0435 E+00 & 1.3076 E-22 & 1.4101 E-22 \\ 47 & 3.0590 E+00 & 6.3111 E-25 & 7.3953 E-25 \\ 48 & 1.8554 E+00 & 2.2438 E-27 & 2.7084 E-27 \\ 49 & 1.1254 E+00 & 3.1457 E-30 & 3.8597 E-30 \\ 50 & 6.8256 E-01 & 2.3559 E-33 & 2.8253 E-33 \\ & 1.0000 E-05 & 1.0000 E+02 & 1.0000 E+02\end{array}$


TABLE $B-X V$

GODIVA CENTRAL HDJOINT SPECTRA

\begin{tabular}{|c|c|c|c|}
\hline GROUP & $\begin{array}{r}\text { ENERGY } \\
\text { BOUNDRRY } \\
\text { (EVI }\end{array}$ & $\begin{array}{r}\text { DIFFUSION } \\
\text { THEORY } \\
\text { [RHO/N/SI }\end{array}$ & $\begin{array}{r}\text { TRANSPORT } \\
\text { THEORY } \\
\text { (RHO/N/S) }\end{array}$ \\
\hline $\begin{array}{r}1 \\
2 \\
3 \\
4 \\
5 \\
6 \\
7 \\
8 \\
9 \\
10 \\
11 \\
12 \\
13 \\
14 \\
15 \\
16 \\
17 \\
18 \\
19 \\
20 \\
21 \\
22 \\
23 \\
24 \\
25 \\
26 \\
27 \\
28 \\
29 \\
30 \\
31 \\
32 \\
33 \\
34 \\
35 \\
36 \\
37 \\
38 \\
39 \\
40 \\
41 \\
42 \\
43 \\
44 \\
45 \\
46 \\
47 \\
48 \\
49 \\
50\end{array}$ & $\begin{array}{l}1.9971 E+07 \\
1.0000 E+07 \\
6.0653 E+06 \\
3.6788 E+06 \\
2.2313 E+06 \\
1.3534 E+06 \\
8.2085 E+05 \\
4.9787 E+05 \\
3.8774 E+05 \\
3.0197 E+05 \\
2.3518 E+05 \\
1.8316 E+05 \\
1.4264 E+05 \\
1.1109 E+05 \\
8.6517 E+04 \\
6.7379 E+04 \\
5.2475 E+04 \\
4.0868 E+04 \\
3.1828 E+04 \\
2.4788 E+04 \\
1.9305 E+04 \\
1.5034 E+04 \\
1.1709 E+04 \\
9.1188 E+03 \\
7.1017 E+03 \\
5.5308 E+03 \\
4.3074 E+03 \\
3.3546 E+03 \\
2.6126 E+03 \\
2.0347 E+03 \\
1.5846 E+03 \\
1.2341 E+03 \\
9.6112 E+02 \\
7.4852 E+02 \\
5.8295 E+02 \\
4.5400 E+02 \\
3.5358 E+02 \\
2.7536 E+02 \\
1.6702 E+02 \\
1.0130 E+02 \\
6.1442 E+01 \\
3.7267 E+01 \\
2.2603 E+01 \\
1.3710 E+01 \\
8.3153 E+00 \\
5.0435 E+00 \\
3.0590 E+00 \\
1.8554 E+00 \\
1.1254 E+00 \\
6.8256 E-01 \\
1.0000 E-05\end{array}$ & $\begin{array}{l}1.5862 E+00 \\
1.2705 E+00 \\
9.7718 E-01 \\
9.9492 E-01 \\
9.7797 E-01 \\
9.4692 E-01 \\
9.5100 E-01 \\
1.0084 E+00 \\
1.0495 E+00 \\
1.0935 E+00 \\
1.1333 E+00 \\
1.1927 E+00 \\
1.2427 E+00 \\
1.2798 E+00 \\
1.3225 E+00 \\
1.3547 E+00 \\
1.3570 E+00 \\
1.3775 E+00 \\
1.3977 E+00 \\
1.3579 E+00 \\
1.3820 E+00 \\
1.4201 E+00 \\
1.4224 E+00 \\
1.4204 E+00 \\
1.4972 E+00 \\
1.5804 E+00 \\
1.6213 E+00 \\
1.6187 E+00 \\
1.5958 E+00 \\
1.6177 E+00 \\
1.6069 E+00 \\
1.4953 E+00 \\
1.4979 E+00 \\
1.6470 E+00 \\
1.7245 E+00 \\
1.6662 E+00 \\
1.5840 E+00 \\
1.6182 E+00 \\
1.5241 E+00 \\
1.6043 E+00 \\
1.7324 E+00 \\
1.6164 E+00 \\
1.5005 E+00 \\
1.6185 E+00 \\
1.0634 E+00 \\
1.3111 E+00 \\
1.6913 E+00 \\
1.8922 E+00 \\
2.1943 E+00 \\
2.1092 E+00 \\
7.1746 E+01\end{array}$ & $\begin{array}{l}1.7158 E+00 \\
1.3465 E+00 \\
1.0072 E+00 \\
1.0094 E+00 \\
9.8364 E-01 \\
9.4395 E-01 \\
9.3179 E-01 \\
9.7319 E-01 \\
1.0047 E+00 \\
1.0402 E+00 \\
1.0723 E+00 \\
1.1228 E+00 \\
1.1658 E+00 \\
1.1983 E+00 \\
1.2375 E+00 \\
1.2675 E+00 \\
1.2688 E+00 \\
1.2896 E+00 \\
1.3133 E+00 \\
1.3100 E+00 \\
1.3332 E+00 \\
1.3741 E+00 \\
1.3825 E+00 \\
1.3826 E+00 \\
1.4620 E+00 \\
1.5403 E+00 \\
1.5868 E+00 \\
1.5875 E+00 \\
1.5686 E+00 \\
1.6013 E+00 \\
1.5964 E+00 \\
1.4954 E+00 \\
1.4959 E+00 \\
1.6660 E+00 \\
1.7634 E+00 \\
1.6764 E+00 \\
1.5962 E+00 \\
1.6265 E+00 \\
1.5402 E+00 \\
1.5823 E+00 \\
1.6736 E+00 \\
1.5639 E+00 \\
1.4521 E+00 \\
1.5636 E+00 \\
1.0284 E+00 \\
1.2694 E+00 \\
1.6289 E+00 \\
1.8252 E+00 \\
2.1171 E+00 \\
2.0340 E+00 \\
7.0216 E+01\end{array}$ \\
\hline
\end{tabular}


TABLE B-XVI

VERA-18 CENTRAL FLUX SPECTRA

\begin{tabular}{|c|c|c|c|c|}
\hline GRGUP & $\begin{array}{r}\text { ENERGY } \\
\text { BOUNDRRY } \\
\text { (EV) }\end{array}$ & $\begin{array}{r}1-0 \\
\text { DIFFUSION } \\
\text { THEORY } \\
\text { (N/CML /S) }\end{array}$ & $\begin{array}{r}1-D \\
\text { TRANSPORT } \\
\text { THEORY } \\
\text { (N/CM2/S) }\end{array}$ & $\begin{array}{r}2-D \\
\text { DIFFUSION } \\
\text { THEOPY } \\
\text { (N/CM } 2 / S \text { ) }\end{array}$ \\
\hline $\begin{array}{l}1 \\
2 \\
3 \\
4 \\
5 \\
6 \\
7 \\
8 \\
9 \\
10 \\
11 \\
12 \\
13 \\
14 \\
15 \\
16 \\
17 \\
18 \\
19 \\
20 \\
21 \\
22 \\
23 \\
24 \\
25 \\
26 \\
27 \\
28 \\
29 \\
30 \\
31 \\
32 \\
33 \\
34 \\
35 \\
36 \\
37 \\
38 \\
39 \\
40 \\
41 \\
42 \\
43 \\
44 \\
45 \\
46 \\
47 \\
48 \\
49 \\
50\end{array}$ & $\begin{array}{l}1.9971 E+07 \\
1.0000 E+07 \\
6.0653 E+06 \\
3.6788 E+06 \\
2.2313 E+06 \\
1.3534 E+06 \\
8.2085 E+05 \\
4.9787 E+05 \\
3.8774 E+05 \\
3.0197 E+05 \\
2.3518 E+05 \\
1.8316 E+05 \\
1.4264 E+05 \\
1.1109 E+05 \\
8.6517 E+04 \\
6.7379 E+04 \\
5.2475 E+04 \\
4.0868 E+04 \\
3.1828 E+04 \\
2.4788 E+04 \\
1.9305 E+04 \\
1.5034 E+04 \\
1.1709 E+04 \\
9.1188 E+03 \\
7.1017 E+03 \\
5.5308 E+03 \\
4.3074 E+03 \\
3.3546 E+03 \\
2.6126 E+03 \\
2.0347 E+03 \\
1.5846 E+03 \\
1.2341 E+03 \\
9.6112 E+02 \\
7.4852 E+02 \\
5.8295 E+02 \\
4.5400 E+02 \\
3.5358 E+02 \\
2.7536 E+02 \\
1.6702 E+02 \\
1.0130 E+02 \\
6.1442 E+01 \\
3.7267 E+01 \\
2.2603 E+01 \\
1.3710 E+01 \\
8.3153 E+00 \\
5.0435 E+00 \\
3.0590 E+00 \\
1.8554 E+00 \\
1.1254 E+00 \\
6.8256 E-01 \\
1.0000 E-05\end{array}$ & $\begin{array}{l}4.9335 E-02 \\
5.6612 E-01 \\
3.1309 E+00 \\
8.0221 E+00 \\
1.1852 E+01 \\
1.2360 E+01 \\
1.1378 E+01 \\
5.6611 E+00 \\
5.3289 E+00 \\
4.9013 E+00 \\
4.4803 E+00 \\
4.0951 E+00 \\
3.7117 E+00 \\
3.3261 E+00 \\
2.9912 E+00 \\
2.6821 E+00 \\
2.3800 E+00 \\
2.0310 E+00 \\
1.7715 E+00 \\
1.6162 E+00 \\
1.3594 E+00 \\
1.1822 E+00 \\
9.9286 E-01 \\
8.1021 E-01 \\
6.6913 E-01 \\
5.4048 E-01 \\
4.2671 E-01 \\
3.3121 E-01 \\
2.4828 E-01 \\
1.7698 E-01 \\
1.2405 E-01 \\
8.0482 E-02 \\
5.2398 E-02 \\
3.1484 E-02 \\
1.7874 E-02 \\
1.0505 E-02 \\
5.9133 E-03 \\
4.0922 E-03 \\
1.1397 E-03 \\
3.1885 E-04 \\
5.5302 E-05 \\
9.6596 E-06 \\
1.8300 E-06 \\
2.7217 E-07 \\
5.5766 E-08 \\
1.5894 E-08 \\
5.3049 E-09 \\
1.3179 E-09 \\
1.5187 E-10 \\
6.2347 E-12 \\
1.0000 E+02\end{array}$ & $\begin{array}{l}5.1945 E-02 \\
5.8373 E-01 \\
3.1826 E+00 \\
8.0744 E+00 \\
1.2027 E+01 \\
1.2506 E+01 \\
1.2059 E+01 \\
5.6861 E+00 \\
5.3412 E+00 \\
4.9005 E+00 \\
4.4673 E+00 \\
4.0713 E+00 \\
3.6801 E+00 \\
3.2886 E+00 \\
2.9507 E+00 \\
2.6392 E+00 \\
2.3359 E+00 \\
1.9884 E+00 \\
1.7334 E+00 \\
1.5771 E+00 \\
1.3242 E+00 \\
1.1493 E+00 \\
9.6339 E-01 \\
7.8521 E-01 \\
6.4774 E-01 \\
5.2274 E-01 \\
4.1252 E-01 \\
3.2008 E-01 \\
2.3999 E-01 \\
1.7124 E-01 \\
1.2015 E-01 \\
7.8070 E-02 \\
5.0920 E-02 \\
3.0664 E-02 \\
1.7453 E-02 \\
1.0285 E-02 \\
5.8068 E-03 \\
4.0396 E-03 \\
1.1317 E-03 \\
3.1836 E-04 \\
5.5359 E-05 \\
9.7042 E-06 \\
1.8458 E-06 \\
2.7534 E-07 \\
5.6675 E-08 \\
1.6227 E-08 \\
5.4325 E-09 \\
1.3534 E-09 \\
1.5608 E-10 \\
6.4101 E-12 \\
1.0000 E+02\end{array}$ & $\begin{array}{l}4.9093 E-02 \\
5.6368 E-01 \\
3.1122 E+00 \\
7.9612 E+00 \\
1.1756 E+01 \\
1.2282 E+01 \\
1.1946 E+01 \\
5.6563 E+00 \\
5.3348 E+00 \\
4.9156 E+00 \\
4.5002 E+00 \\
4.1184 E+00 \\
3.7367 E+00 \\
3.3513 E+00 \\
3.0161 E+00 \\
2.7064 E+00 \\
2.4031 E+00 \\
2.0517 E+00 \\
1.7899 E+00 \\
1.6327 E+00 \\
1.3728 E+00 \\
1.1932 E+00 \\
1.0013 E+00 \\
8.1640 E-01 \\
6.7359 E-01 \\
5.4353 E-01 \\
4.2869 E-01 \\
3.3241 E-01 \\
2.4895 E-01 \\
1.7730 E-01 \\
1.2417 E-01 \\
8.0502 E-02 \\
5.2371 E-02 \\
3.1446 E-02 \\
1.7841 E-02 \\
1.0479 E-02 \\
5.8951 E-03 \\
4.0771 E-03 \\
1.1349 E-03 \\
3.1732 E-04 \\
5.5023 E-05 \\
9.6083 E-06 \\
1.8197 E-06 \\
2.7058 E-0 \\
5.5418 E-08 \\
1.5784 E-08 \\
5.2632 E-09 \\
1.3066 E-09 \\
1.5054 E-10 \\
6.1796 E-12 \\
1.0000 E+02\end{array}$ \\
\hline
\end{tabular}


TABLE B-XVII

VERA-1B EDGE FLUX SPECTRA

\begin{tabular}{|c|c|c|c|c|}
\hline SROUP & $\begin{array}{r}\text { ENCRGY } \\
\text { BOUNDRRY } \\
\text { (EV) }\end{array}$ & $\begin{array}{r}1-D \\
\text { DIFFUSION } \\
\text { THEORY } \\
\text { (N/CM } 2 / S)\end{array}$ & $\begin{array}{r}\text { I D } \\
\text { TRANSPORT } \\
\text { THEORY } \\
\text { [N/CM } 2 / S \text { ] }\end{array}$ & $\begin{array}{r}2-0 \\
\text { OIFFUSION } \\
\text { THEORY } \\
\text { (N/CM2/S) }\end{array}$ \\
\hline $\begin{array}{r}1 \\
2 \\
3 \\
4 \\
5 \\
6 \\
7 \\
8 \\
9 \\
10 \\
11 \\
12 \\
13 \\
14 \\
15 \\
16 \\
17 \\
18 \\
19 \\
20 \\
21 \\
22 \\
23 \\
24 \\
25 \\
26 \\
27 \\
28 \\
29 \\
30 \\
31 \\
32 \\
33 \\
34 \\
35 \\
36 \\
37 \\
38 \\
39 \\
40 \\
41 \\
42 \\
43 \\
44 \\
45 \\
46 \\
47 \\
48 \\
49 \\
50 \\
\end{array}$ & $\begin{array}{l}1.9971 E+07 \\
1.0000 E+07 \\
6.0653 E+06 \\
3.6783 E+06 \\
2.2313 E+06 \\
1.3534 E+06 \\
8.2085 E+05 \\
4.9787 E+05 \\
3.8774 E+05 \\
3.0197 E+05 \\
2.3518 E+05 \\
1.8316 E+05 \\
1.4264 E+05 \\
1.1109 E+05 \\
8.6517 E+04 \\
6.7379 E+04 \\
5.2475 E+04 \\
4.0868 E+04 \\
3.1828 E+04 \\
2.4788 E+04 \\
1.9305 E+04 \\
1.5034 E+04 \\
1.1709 E+04 \\
9.1188 E+03 \\
7.1017 E+03 \\
5.5308 E+03 \\
4.3074 E+03 \\
3.3546 E+03 \\
2.6126 E+03 \\
2.0347 E+03 \\
1.5846 E+03 \\
1.2341 E+03 \\
9.6112 E+02 \\
7.4852 E+02 \\
5.8295 E+02 \\
4.5400 E+02 \\
3.5358 E+02 \\
2.7536 E+02 \\
1.6702 E+02 \\
1.0130 E+02 \\
6.1442 E+01 \\
3.7267 E+01 \\
2.2603 E+01 \\
1.3710 E+01 \\
8.3153 E+00 \\
5.0435 E+00 \\
3.0590 E+00 \\
1.8554 E+00 \\
1.1254 E+00 \\
6.8256 E-01 \\
1.0000 E-05\end{array}$ & $\begin{array}{l}6.9360 E-03 \\
7.9899 E-02 \\
3.1076 E-01 \\
6.4032 E-01 \\
1.0003 E+00 \\
2.6967 E+00 \\
9.9760 E+00 \\
6.7032 E+00 \\
8.0959 E+00 \\
8.2320 E+00 \\
7.7116 E+00 \\
7.2934 E+00 \\
7.0607 E+00 \\
6.6856 E+00 \\
6.7012 E+00 \\
6.2541 E+00 \\
6.1282 E+00 \\
3.9687 E+00 \\
2.4153 E+00 \\
3.4239 E+00 \\
1.4561 F+00 \\
1.1318 E+00 \\
8.2049 E-01 \\
3.8560 E-01 \\
2.8287 E-01 \\
1.6136 E-01 \\
1.2876 E-01 \\
6.9450 E-02 \\
4.5274 E-02 \\
3.7073 E-02 \\
1.7907 E-02 \\
7.4764 E-03 \\
5.1922 E-03 \\
2.4244 E-03 \\
1.4748 E-03 \\
8.1295 E-04 \\
5.4409 E-04 \\
5.7800 E-04 \\
9.1678 E-05 \\
1.8789 E-05 \\
3.3626 E-06 \\
3.8706 E-07 \\
2.3139 E-00 \\
3.7303 E-09 \\
7.6375 E-11 \\
3.4558 E-11 \\
4.9031 E-11 \\
3.0006 E-11 \\
6.7183 E-12 \\
4.3812 E-13 \\
1.0000 E+02\end{array}$ & $\begin{array}{l}8.1395 E-03 \\
8.8685 E-02 \\
3.4281 E-01 \\
7.0039 E-01 \\
1.1049 E+00 \\
2.9198 E+00 \\
1.0040 E+01 \\
6.7060 E+00 \\
8.0485 E+00 \\
8.2444 E+00 \\
7.6887 E+00 \\
7.2847 E+00 \\
7.0562 E+00 \\
6.7179 E+00 \\
6.7273 E+00 \\
6.3122 E+00 \\
6.0977 E+00 \\
3.8870 E+00 \\
2.3372 E+00 \\
3.2926 E+00 \\
1.4168 E+00 \\
1.1043 E+00 \\
7.9305 E-01 \\
3.7642 E-01 \\
2.7462 E-01 \\
1.5671 E-01 \\
1.2219 E-01 \\
6.6419 E-02 \\
3.6800 E-02 \\
2.1807 E-02 \\
1.2231 E-02 \\
5.1063 E-03 \\
3.5268 E-03 \\
1.7226 E-03 \\
1.1400 E-03 \\
6.8848 E-04 \\
4.5866 E-04 \\
3.4183 E-04 \\
6.5267 E-05 \\
1.2562 E-05 \\
2.5301 E-06 \\
2.9103 E-07 \\
1.7298 E-00 \\
3.1766 E-09 \\
6.2263 E-11 \\
3.8007 E-11 \\
6.1399 E-11 \\
3.7279 E-11 \\
8.2623 E-12 \\
5.3815 E-13 \\
1.0000 E+02\end{array}$ & $\begin{array}{l}5.9977 E-03 \\
7.0089 E-02 \\
2.7535 E-01 \\
5.7233 E-01 \\
8.9507 E-01 \\
2.3853 E+00 \\
9.0541 E+00 \\
6.2461 E+00 \\
7.6590 E+00 \\
7.9921 E+00 \\
7.6005 E+00 \\
7.2967 E+00 \\
7.1576 E+00 \\
6.9110 E+00 \\
7.0321 E+00 \\
6.6395 E+00 \\
6.5408 E+00 \\
4.2622 E+00 \\
2.6807 E+00 \\
3.6822 E+00 \\
1.6157 E+00 \\
1.2561 E+00 \\
9.0028 E-01 \\
4.3295 E-01 \\
3.1527 E-01 \\
1.8006 E-01 \\
1.4304 E-01 \\
7.6829 E-02 \\
4.7385 E-02 \\
3.6319 E-02 \\
1.8387 E-02 \\
7.6862 E-03 \\
5.1601 E-03 \\
2.4520 E-03 \\
1.4825 E-03 \\
8.3274 E-04 \\
5.4588 E-04 \\
5.4099 E-04 \\
9.2719 E-05 \\
1.7866 E-05 \\
3.4382 E-06 \\
3.8078 E-00 \\
2.2763 E-08 \\
3.7383 E-09 \\
7.8471 E-11 \\
3.3146 E-11 \\
4.5674 E-11 \\
2.8143 E-11 \\
6.3616 E-12 \\
4.3159 E-13 \\
1.0000 E+02\end{array}$ \\
\hline
\end{tabular}


TABLE B-XVIII

VERA-IB CENTRAL ADJOINT SFECTRA

\begin{tabular}{|c|c|c|c|c|}
\hline GROUP & $\begin{array}{r}\text { ENERGY } \\
\text { BOUNDRRY } \\
\text { (EV) }\end{array}$ & $\begin{array}{r}1-0 \\
\text { OIFFUSION } \\
\text { THEORY } \\
\text { (RHO/N/S) }\end{array}$ & $\begin{array}{r}1-0 \\
\text { TRANSPORT } \\
\text { THEORY } \\
\text { IRHO/N/SI }\end{array}$ & $\begin{array}{r}2-D \\
\text { DIFFUSION } \\
\text { THEORY } \\
\text { IRHO/N/SI }\end{array}$ \\
\hline $\begin{array}{l}1 \\
2 \\
3 \\
4 \\
5 \\
6 \\
7 \\
8 \\
9 \\
10 \\
11 \\
12 \\
13 \\
14 \\
15 \\
16 \\
17 \\
18 \\
19 \\
20 \\
21 \\
22 \\
23 \\
24 \\
25 \\
26 \\
27 \\
28 \\
29 \\
30 \\
31 \\
32 \\
33 \\
34 \\
35 \\
36 \\
37 \\
38 \\
39 \\
40 \\
41 \\
42 \\
43 \\
44 \\
45 \\
46 \\
47 \\
48 \\
49 \\
50\end{array}$ & $\begin{array}{l}1.9971 E+07 \\
1.0000 E+07 \\
6.0653 E+06 \\
3.6788 E+06 \\
2.2313 E+06 \\
1.3534 E+06 \\
8.2085 E+05 \\
4.9787 E+05 \\
3.8774 E+05 \\
3.0197 E+05 \\
2.3518 E+05 \\
1.8316 E+05 \\
1.4264 E+05 \\
1.1109 E+05 \\
8.6517 E+04 \\
6.7379 E+04 \\
5.2475 E+04 \\
4.0868 E+04 \\
3.1828 E+04 \\
2.4788 E+04 \\
1.9305 E+04 \\
1.5034 E+04 \\
1.1709 E+04 \\
9.1188 E+03 \\
7.1017 E+03 \\
5.5308 E+03 \\
4.3074 E+03 \\
3.3546 E+03 \\
2.6126 E+03 \\
2.0347 E+03 \\
1.5846 E+03 \\
1.2341 E+03 \\
9.6112 E+02 \\
7.4852 E+02 \\
5.8295 E+02 \\
4.5400 E+02 \\
3.5358 E+02 \\
2.7536 E+02 \\
1.6702 E+02 \\
1.0130 E+02 \\
6.1442 E+01 \\
3.7267 E+01 \\
2.2603 E+01 \\
1.3710 E+01 \\
8.3153 E+00 \\
5.0435 E+00 \\
3.0590 E+00 \\
1.8554 E+00 \\
1.1254 E+00 \\
6.8256 E-01 \\
1.0000 E-05\end{array}$ & $\begin{array}{l}1.1819 E+00 \\
9.9962 E-01 \\
9.3914 E-01 \\
9.6111 E-01 \\
9.4253 E-01 \\
9.7585 E-01 \\
1.0438 E+00 \\
1.1212 E+00 \\
1.1556 E+00 \\
1.1900 E+00 \\
1.2216 E+00 \\
1.2491 E+00 \\
1.2747 E+00 \\
1.3007 E+00 \\
1.3238 E+00 \\
1.3448 E+00 \\
1.3663 E+00 \\
1.3908 E+00 \\
1.4126 E+00 \\
1.4251 E+00 \\
1.4558 E+00 \\
1.4769 E+00 \\
1.4981 E+00 \\
1.5276 E+00 \\
1.5574 E+00 \\
1.5816 E+00 \\
1.5915 E+00 \\
1.5912 E+00 \\
1.5910 E+00 \\
1.5978 E+00 \\
1.5937 E+00 \\
1.5806 E+00 \\
1.6390 E+00 \\
1.7030 E+00 \\
1.7114 E+00 \\
1.6493 E+00 \\
1.6144 E+00 \\
1.6103 E+00 \\
1.5684 E+00 \\
1.6092 E+00 \\
1.6703 E+00 \\
1.5619 E+00 \\
1.4720 E+00 \\
1.4717 E+00 \\
9.9782 E-01 \\
1.4021 E+00 \\
1.6779 E+00 \\
1.9113 E+00 \\
2.1512 E+00 \\
2.0907 E+00 \\
7.1975 E+01\end{array}$ & $\begin{array}{l}1.1992 E+00 \\
1.0266 E+00 \\
9.5529 E-01 \\
9.6785 E-01 \\
9.4937 E-01 \\
9.7317 E-01 \\
1.0301 E+00 \\
1.1002 E+00 \\
1.1322 E+00 \\
1.1644 E+00 \\
1.1943 E+00 \\
1.2205 E+00 \\
1.2449 E+00 \\
1.2696 E+00 \\
1.2919 E+00 \\
1.3116 E+00 \\
1.3323 E+00 \\
1.3563 E+00 \\
1.3780 E+00 \\
1.3871 E+00 \\
1.4171 E+00 \\
1.4371 E+00 \\
1.4579 E+00 \\
1.4869 E+00 \\
1.5159 E+00 \\
1.5399 E+00 \\
1.5499 E+00 \\
1.5501 E+00 \\
1.5507 E+00 \\
1.5581 E+00 \\
1.5549 E+00 \\
1.5430 E+00 \\
1.6006 E+00 \\
1.6637 E+00 \\
1.6724 E+00 \\
1.6120 E+00 \\
1.5786 E+00 \\
1.5753 E+00 \\
1.5346 E+00 \\
1.5728 E+00 \\
1.6310 E+00 \\
1.5262 E+00 \\
1.4383 E+00 \\
1.4376 E+00 \\
9.7509 E-01 \\
1.3690 E+00 \\
1.6364 E+00 \\
1.8648 E+00 \\
2.0983 E+00 \\
2.0393 E+00 \\
7.0472 E+01\end{array}$ & $\begin{array}{l}1.1935 E+00 \\
1.0072 E+00 \\
9.4378 E-01 \\
9.6429 E-01 \\
9.4374 E-01 \\
9.7365 E-01 \\
1.0400 E+00 \\
1.1163 E+00 \\
1.1501 E+00 \\
1.1839 E+00 \\
1.2148 E+00 \\
1.2417 E+00 \\
1.2667 E+00 \\
1.2922 E+00 \\
1.3149 E+00 \\
1.3357 E+00 \\
1.3569 E+00 \\
1.3813 E+00 \\
1.4032 E+00 \\
1.4157 E+00 \\
1.4465 E+00 \\
1.4678 E+00 \\
1.4892 E+00 \\
1.5190 E+00 \\
1.5488 E+00 \\
1.5734 E+00 \\
1.5835 E+00 \\
1.5837 E+00 \\
1.5840 E+00 \\
1.5912 E+00 \\
1.5874 E+00 \\
1.5745 E+00 \\
1.6328 E+00 \\
1.6965 E+00 \\
1.7049 E+00 \\
1.6431 E+00 \\
1.6083 E+00 \\
1.6042 E+00 \\
1.5625 E+00 \\
1.6031 E+00 \\
1.6638 E+00 \\
1.5559 E+00 \\
1.4663 E+00 \\
1.4660 E+00 \\
9.9400 E-01 \\
1.3969 E+00 \\
1.6717 E+00 \\
1.9039 E+00 \\
2.1426 E+00 \\
2.0822 E+00 \\
7.1687 E+01\end{array}$ \\
\hline
\end{tabular}


TABLE $B-X I X$

ZPR-3-6F CENTRAL FLUX SPECTRA

\begin{tabular}{|c|c|c|c|c|}
\hline ¿ROUP & $\begin{array}{r}\text { ENERGYY } \\
\text { BOUNDARY } \\
\text { (EV) }\end{array}$ & $\begin{array}{r}1-D \\
\text { DIFFUSION } \\
\text { THEORY } \\
\text { (N/CMZ/S) }\end{array}$ & $\begin{array}{r}1-0 \\
\text { TRANSPORT } \\
\text { THEORY } \\
\text { IN/CM2/SI }\end{array}$ & $\begin{array}{r}2-0 \\
\text { OIFFUSION } \\
\text { THEORY } \\
\text { (N/CMZ/SI }\end{array}$ \\
\hline $\begin{array}{r}1 \\
2 \\
3 \\
4 \\
5 \\
6 \\
7 \\
8 \\
9 \\
10 \\
11 \\
12 \\
13 \\
14 \\
15 \\
16 \\
17 \\
18 \\
19 \\
20 \\
21 \\
22 \\
23 \\
24 \\
25 \\
26 \\
27 \\
28 \\
29 \\
30 \\
31 \\
32 \\
33 \\
34 \\
35 \\
36 \\
37 \\
38 \\
39 \\
40 \\
41 \\
42 \\
43 \\
44 \\
45 \\
46 \\
47 \\
48 \\
49 \\
50\end{array}$ & $\begin{array}{l}1.9971 E+07 \\
1.0000 E+07 \\
6.0653 E+06 \\
3.6788 E+06 \\
2.2313 E+06 \\
1.3534 E+06 \\
8.2085 E+05 \\
4.9787 E+05 \\
3.8774 E+05 \\
3.0197 E+05 \\
2.3518 E+05 \\
1.8316 E+05 \\
1.4264 E+05 \\
1.1109 E+05 \\
8.6517 E+04 \\
6.7379 E+04 \\
5.2475 E+04 \\
4.0868 E+04 \\
3.1828 E+04 \\
2.4788 E+04 \\
1.9305 E+04 \\
1.5034 E+04 \\
1.1709 E+04 \\
9.1188 E+03 \\
7.1017 E+03 \\
5.5308 E+03 \\
4.3074 E+03 \\
3.3546 E+03 \\
2.6126 E+03 \\
2.0347 E+03 \\
1.5846 E+03 \\
1.2341 E+03 \\
9.6112 E+02 \\
7.4852 E+02 \\
5.8295 E+02 \\
4.5400 E+02 \\
3.5358 E+02 \\
2.7536 E+02 \\
1.6702 E+02 \\
1.0130 E+02 \\
6.1442 E+01 \\
3.7267 E+01 \\
2.2603 E+01 \\
1.3710 E+01 \\
8.3153 E+00 \\
5.0435 E+00 \\
3.0590 E+00 \\
1.8554 E+00 \\
1.1254 E+00 \\
6.8256 E-01 \\
1.0000 E-05\end{array}$ & $\begin{array}{l}4.8131 E-0 E \\
6.6928 E-U 1 \\
2.9367 E+00 \\
6.7520 E+00 \\
9.8729 E+00 \\
1.2789 E+01 \\
1.5834 E+01 \\
8.2557 E+00 \\
8.3396 E+00 \\
7.1390 E+00 \\
5.2252 E+00 \\
4.1535 E+00 \\
4.6554 E+00 \\
2.6735 E+00 \\
3.1860 E+00 \\
3.0624 E+00 \\
1.4342 E+00 \\
4.9199 E-01 \\
9.9373 E-01 \\
8.5814 E-01 \\
2.7694 E-01 \\
1.4198 E-01 \\
7.0345 E-02 \\
3.3513 E-02 \\
2.0731 E-02 \\
1.2674 E-02 \\
7.4110 E-03 \\
3.3764 E-03 \\
1.5068 E-03 \\
7.6110 E-04 \\
3.9455 E-04 \\
1.9252 E-04 \\
1.2014 E-04 \\
7.1417 E-05 \\
4.2280 E-05 \\
2.2330 E-05 \\
1.8595 E-05 \\
1.6135 E-05 \\
1.3947 E-05 \\
6.1110 E-07 \\
1.2978 E-08 \\
3.8725 E-10 \\
9.8759 E-12 \\
2.1553 E-13 \\
4.7113 E-15 \\
2.4689 E-16 \\
1.9251 E-17 \\
9.8901 E-19 \\
1.70111 E-20 \\
1.1209 E-22 \\
1.0000 E+02\end{array}$ & $\begin{array}{l}4.8933 E-02 \\
6.7383 E-01 \\
2.9349 E+00 \\
6.7477 E+00 \\
9.8891 E+00 \\
1.2925 E+01 \\
1.6017 E+01 \\
8.2824 E+00 \\
8.3612 E+00 \\
7.1406 E+00 \\
5.2048 E+00 \\
4.1290 E+00 \\
4.6141 E+00 \\
2.6489 E+00 \\
3.1502 E+00 \\
2.9996 E+00 \\
1.4035 E+00 \\
4.7988 E-01 \\
9.6877 E-01 \\
8.2986 E-01 \\
2.6753 E-01 \\
1.3709 E-01 \\
6.7915 E-C 2 \\
3.2395 E-02 \\
2.0026 E-02 \\
1.2256 E-02 \\
7.1677 E-03 \\
3.2681 E-03 \\
1.4589 E-03 \\
7.4188 E-04 \\
3.8651 E-04 \\
1.8898 E-04 \\
1.1824 E-04 \\
7.0305 E-05 \\
4.1646 E-05 \\
2.1963 E-05 \\
1.8276 E-05 \\
1.5919 E-05 \\
1.3747 E-05 \\
6.0524 E-07 \\
1.4852 E-00 \\
3.8473 E-10 \\
9.8333 E-12 \\
2.1521 E-13 \\
4.7096 E-15 \\
2.5325 E-16 \\
2.1863 E-17 \\
1.1697 E-18 \\
2.0130 E-20 \\
1.3220 E-22 \\
1.0000 E+02\end{array}$ & $\begin{array}{l}4.8041 E-02 \\
6.6813 E-01 \\
2.9315 E+00 \\
6.7354 E+00 \\
9.8406 E+00 \\
1.2743 E+01 \\
1.5865 E+01 \\
8.2481 E+00 \\
8.3401 E+00 \\
7.1467 E+00 \\
5.2358 E+00 \\
4.1649 E+00 \\
4.6718 E+00 \\
2.6846 E+00 \\
3.2037 E+00 \\
3.0813 E+00 \\
1.4458 E+00 \\
4.9610 E-01 \\
1.0039 E+00 \\
8.6720 E-01 \\
2.8028 E-01 \\
1.4393 E-01 \\
7.1379 E-02 \\
3.3977 E-02 \\
2.1033 E-02 \\
1.2854 E-02 \\
7.5216 E-03 \\
3.4285 E-03 \\
1.5261 E-03 \\
7.6840 E-04 \\
3.9711 E-04 \\
1.9333 E-04 \\
1.2050 E-04 \\
7.1593 E-05 \\
4.2379 E-05 \\
2.2382 E-05 \\
1.8637 E-05 \\
1.6171 E-05 \\
1.3978 E-05 \\
6.1250 E-07 \\
1.5013 E-08 \\
3.8822 E-10 \\
9.9365 E-12 \\
2.1639 E-13 \\
4.7394 E-15 \\
2.5684 E-16 \\
2.1580 E-17 \\
1.1582 E-18 \\
2.0098 E-20 \\
1.3255 E-22 \\
1.0000 E+02\end{array}$ \\
\hline
\end{tabular}


TABLE $B-X X$

ZPR-3-6F EDGE FLUX SPECTRA

\begin{tabular}{|c|c|c|c|c|}
\hline GROUP & $\begin{array}{r}\text { ENERGY } \\
\text { BOUNDARY } \\
\text { (EV) }\end{array}$ & $\begin{array}{r}1-0 \\
\text { OIFFUSION } \\
\text { THEORY } \\
\text { (N/CM } 2 / S \text { ) }\end{array}$ & $\begin{array}{r}1-0 \\
\text { TRANSPORT } \\
\text { THEORY } \\
\text { (N/CM2/S) }\end{array}$ & $\begin{array}{r}2-D \\
\text { DIFFUSION } \\
\text { THEORY } \\
\text { (N/CM2/S) }\end{array}$ \\
\hline $\begin{array}{l}1 \\
2 \\
3 \\
4 \\
5 \\
6 \\
7 \\
8 \\
9 \\
10 \\
11 \\
12 \\
13 \\
14 \\
15 \\
16 \\
17 \\
18 \\
19 \\
20 \\
21 \\
22 \\
23 \\
24 \\
25 \\
26 \\
27 \\
28 \\
29 \\
30 \\
31 \\
32 \\
33 \\
34 \\
35 \\
36 \\
37 \\
38 \\
39 \\
40 \\
41 \\
42 \\
43 \\
44 \\
45 \\
46 \\
47 \\
48 \\
49 \\
50\end{array}$ & $\begin{array}{l}1.9971 E+07 \\
1.0000 E+07 \\
6.0653 E+06 \\
3.6788 E+06 \\
2.2313 E+06 \\
1.3534 E+06 \\
8.2085 E+05 \\
4.9787 E+05 \\
3.8774 E+05 \\
3.0197 E+05 \\
2.3518 E+05 \\
1.8316 E+05 \\
1.4264 E+05 \\
1.1109 E+05 \\
8.6517 E+04 \\
6.7379 E+04 \\
5.2475 E+04 \\
4.0868 E+04 \\
3.1828 E+04 \\
2.4788 E+04 \\
1.9305 E+04 \\
1.5034 E+04 \\
1.1709 E+04 \\
9.1188 E+03 \\
7.1017 E+03 \\
5.5308 E+03 \\
4.3074 E+03 \\
3.3546 E+03 \\
2.6126 E+03 \\
2.0347 E+03 \\
1.5846 E+03 \\
1.2341 E+03 \\
9.6112 E+02 \\
7.4852 E+02 \\
5.8295 E+02 \\
4.5400 E+02 \\
3.5358 E+02 \\
2.7536 E+02 \\
1.6702 E+02 \\
1.0130 E+02 \\
6.1442 E+01 \\
3.7267 E+01 \\
2.2603 E+01 \\
1.3710 E+01 \\
8.3153 E+00 \\
5.0435 E+00 \\
3.0590 E+00 \\
1.8554 E+00 \\
1.1254 E+00 \\
6.8256 E-01 \\
1.0000 E-05\end{array}$ & $\begin{array}{l}4.4610 E-03 \\
4.8694 E-02 \\
1.8401 E-01 \\
3.7883 E-01 \\
6.2088 E-01 \\
2.1128 E+00 \\
9.3591 E+00 \\
6.5427 E+00 \\
8.0795 E+00 \\
8.4907 E+00 \\
7.9550 E+00 \\
7.2458 E+00 \\
7.3535 E+00 \\
6.5446 E+00 \\
7.3234 E+00 \\
6.9526 E+00 \\
6.1901 E+00 \\
3.6420 E+00 \\
3.0316 E+00 \\
3.6496 E+00 \\
1.7861 E+00 \\
1.0997 E+00 \\
6.1603 E-01 \\
2.9433 E-01 \\
2.0253 E-01 \\
1.0844 E-01 \\
1.0136 E-01 \\
4.3518 E-02 \\
2.0815 E-02 \\
1.2317 E-02 \\
3.3199 E-03 \\
9.0831 E-04 \\
3.9803 E-04 \\
1.2721 E-04 \\
6.2821 E-05 \\
2.5609 E-05 \\
1.5522 E-05 \\
2.6198 E-05 \\
6.4824 E-06 \\
1.1988 E-06 \\
1.8678 E-07 \\
1.8577 E-08 \\
8.5364 E-10 \\
1.1660 E-10 \\
1.8381 E-12 \\
2.5709 E-13 \\
4.9233 E-14 \\
9.0298 E-15 \\
1.2375 E-15 \\
9.4095 E-17 \\
1.0000 E+02\end{array}$ & $\begin{array}{l}6.2439 E-03 \\
6.6833 E-02 \\
2.4568 E-01 \\
4.9250 E-01 \\
7.9029 E-01 \\
2.4357 E+00 \\
9.4808 E+00 \\
6.5441 E+00 \\
8.0151 E+00 \\
8.4106 E+00 \\
7.8955 E+00 \\
7.2081 E+00 \\
7.3178 E+00 \\
6.5438 E+00 \\
7.3191 E+00 \\
6.9747 E+00 \\
6.1168 E+00 \\
3.5511 E+00 \\
2.9374 E+00 \\
3.5018 E+00 \\
1.7251 E+00 \\
1.0640 E+00 \\
5.9635 E-01 \\
2.8791 E-01 \\
1.9756 E-01 \\
1.0558 E-01 \\
9.6637 E-02 \\
4.1935 E-02 \\
1.7710 E-02 \\
8.7071 E-03 \\
3.0201 E-03 \\
8.6388 E-04 \\
3.7665 E-04 \\
1.2423 E-04 \\
6.2789 E-05 \\
2.6361 E-05 \\
1.5146 E-05 \\
1.4762 E-05 \\
5.3904 E-06 \\
8.3684 E-07 \\
1.4994 E-07 \\
1.4759 E-08 \\
6.7832 E-10 \\
1.0299 E-10 \\
1.5648 E-12 \\
2.3470 E-13 \\
4.6490 E-14 \\
8.7119 E-15 \\
1.2090 E-15 \\
9.2841 E-17 \\
1.0000 E+02\end{array}$ & $\begin{array}{l}4.0610 E-03 \\
4.4665 E-02 \\
1.6985 E-01 \\
3.5176 E-01 \\
5.7771 E-01 \\
1.9648 E+00 \\
8.8669 E+00 \\
6.2950 E+00 \\
7.8431 E+00 \\
8.3278 E+00 \\
7.8977 E+00 \\
7.2643 E+00 \\
7.4213 E+00 \\
6.6797 E+00 \\
7.5196 E+00 \\
7.1767 E+70 \\
6.4022 E+00 \\
3.7754 E+00 \\
3.1808 E+00 \\
3.7668 E+00 \\
1.8569 E+00 \\
1.1445 E+00 \\
6.4130 E-01 \\
3.1023 E-01 \\
2.1288 E-01 \\
1.1383 E-01 \\
1.0620 E-01 \\
4.5695 E-02 \\
2.1228 E-02 \\
1.2017 E-02 \\
3.4072 E-03 \\
9.4120 E-04 \\
4.0645 E-04 \\
1.3207 E-04 \\
6.5103 E-05 \\
2.6908 E-05 \\
1.6123 E-05 \\
2.5052 E-05 \\
6.5803 E-06 \\
1.1603 E-06 \\
1.9195 E-07 \\
1.8640 E-08 \\
8.5965 E-10 \\
1.2004 E-10 \\
1.9262 E-12 \\
2.6449 E-13 \\
5.1005 E-14 \\
9.4299 E-15 \\
1.3013 E-15 \\
1.0080 E-16 \\
1.0000 E+02\end{array}$ \\
\hline
\end{tabular}


TABLE $B-x \times 1$

ZPR-3-6F CENTRAL ADJOINT SPECTRA

\begin{tabular}{|c|c|c|c|c|}
\hline GROUP & $\begin{array}{r}\text { ENERGY } \\
\text { BOUNORRY } \\
\text { (EV) }\end{array}$ & $\begin{array}{r}1-0 \\
\text { DIFFUSION } \\
\text { THEORY } \\
\text { (RHO/N/S) }\end{array}$ & $\begin{array}{r}1-D \\
\text { TRANSPOIRT } \\
\text { THEORY } \\
\text { (RHO/N/S) }\end{array}$ & $\begin{array}{r}2-D \\
\text { DIFFUSION } \\
\text { THEORY } \\
\text { (RHO/N/S) }\end{array}$ \\
\hline $\begin{array}{r}1 \\
2 \\
3 \\
4 \\
5 \\
6 \\
7 \\
8 \\
9 \\
10 \\
11 \\
12 \\
13 \\
14 \\
15 \\
16 \\
17 \\
18 \\
19 \\
20 \\
21 \\
22 \\
23 \\
24 \\
25 \\
26 \\
27 \\
28 \\
29 \\
30 \\
31 \\
32 \\
33 \\
34 \\
35 \\
36 \\
37 \\
38 \\
39 \\
40 \\
41 \\
42 \\
43 \\
44 \\
45 \\
46 \\
47 \\
48 \\
49 \\
50\end{array}$ & $\begin{array}{l}1.9971 E+07 \\
1.0000 E+07 \\
6.0653 E+06 \\
3.6788 E+06 \\
2.2313 E+06 \\
1.3534 E+06 \\
8.2085 E+05 \\
4.9787 E+05 \\
3.8774 E+05 \\
3.0197 E+05 \\
2.3518 E+05 \\
1.8316 E+05 \\
1.4264 E+05 \\
1.1109 E+05 \\
8.6517 E+04 \\
6.7379 E+04 \\
5.2475 E+04 \\
4.0868 E+04 \\
3.1828 E+04 \\
2.4788 E+04 \\
1.9305 E+04 \\
1.5034 E+04 \\
1.1709 E+04 \\
9.1188 E+03 \\
7.1017 E+03 \\
5.5308 E+03 \\
4.3074 E+03 \\
3.3546 E+03 \\
2.6126 E+03 \\
2.0347 E+03 \\
1.5846 E+03 \\
1.2341 E+03 \\
9.6112 E+02 \\
7.4852 E+02 \\
5.8295 E+02 \\
4.5400 E+02 \\
3.5358 E+02 \\
2.7536 E+02 \\
1.6702 E+02 \\
1.0130 E+02 \\
6.1442 E+01 \\
3.7267 E+01 \\
2.2603 E+01 \\
1.3710 E+01 \\
8.3153 E+00 \\
5.0435 E+00 \\
3.0590 E+00 \\
1.8554 E+00 \\
1.1254 E+00 \\
6.8256 E-01 \\
1.0000 E-05\end{array}$ & $\begin{array}{l}1.5661 E+00 \\
1.2827 E+00 \\
1.0483 E+00 \\
1.0478 E+00 \\
9.9496 E-01 \\
9.0714 E-01 \\
9.0993 E-01 \\
9.7012 E-01 \\
9.8281 E-01 \\
1.0212 E+00 \\
1.0789 E+00 \\
1.1058 E+00 \\
1.0948 E+00 \\
1.1284 E+00 \\
1.1148 E+00 \\
1.1042 E+00 \\
1.1244 E+00 \\
1.1370 E+00 \\
1.1101 E+00 \\
1.0634 E+00 \\
1.1251 E+00 \\
1.1629 E+00 \\
1.1928 E+00 \\
1.2506 E+00 \\
1.2987 E+00 \\
1.3580 E+00 \\
1.3980 E+00 \\
1.3900 E+00 \\
1.3962 E+00 \\
1.4221 E+00 \\
1.4298 E+00 \\
1.3325 E+00 \\
1.3954 E+00 \\
1.5528 E+00 \\
1.6367 E+00 \\
1.5486 E+00 \\
1.4957 E+00 \\
1.5209 E+00 \\
1.4220 E+00 \\
1.4413 E+00 \\
1.6155 E+00 \\
1.4526 E+00 \\
1.2819 E+00 \\
1.4951 E+00 \\
5.4637 E-01 \\
1.2195 E+00 \\
1.5073 E+00 \\
1.7638 E+00 \\
2.0881 E+00 \\
2.0216 E+00 \\
5.4655 E+01\end{array}$ & $\begin{array}{l}1.5806 E+00 \\
1.3925 E+00 \\
1.0545 E+00 \\
1.0503 E+00 \\
9.9563 E-01 \\
9.0921 E-01 \\
9.0601 E-01 \\
9.5986 E-01 \\
9.7396 E-01 \\
1.0097 E+00 \\
1.0630 E+00 \\
1.0918 E+00 \\
1.0822 E+00 \\
1.1157 E+00 \\
1.1015 E+00 \\
1.0843 E+00 \\
1.1108 E+00 \\
1.1335 E+00 \\
1.1094 E+00 \\
1.0498 E+00 \\
1.1070 E+00 \\
1.1422 E+00 \\
1.1712 E+00 \\
1.2268 E+00 \\
1.2748 E+00 \\
1.3325 E+00 \\
1.3729 E+00 \\
1.3655 E+00 \\
1.3745 E+00 \\
1.4017 E+00 \\
1.4065 E+00 \\
1.3118 E+00 \\
1.3746 E+00 \\
1.5299 E+00 \\
1.6130 E+00 \\
1.5237 E+00 \\
1.4725 E+00 \\
1.5008 E+00 \\
1.4034 E+00 \\
1.4202 E+00 \\
1.5864 E+00 \\
1.4283 E+00 \\
1.2606 E+00 \\
1.4687 E+00 \\
6.3545 E-01 \\
1.1985 E+00 \\
1.4776 E+00 \\
1.7305 E+00 \\
2.0478 E+00 \\
1.9826 E+00 \\
6.3816 E+01\end{array}$ & $\begin{array}{l}1.5715 E+00 \\
1.2857 E+00 \\
1.0496 E+00 \\
1.0489 E+00 \\
9.9515 E-01 \\
9.0617 E-01 \\
9.0878 E-01 \\
9.6882 E-01 \\
9.8147 E-01 \\
1.0196 E+00 \\
1.0771 E+00 \\
1.1042 E+00 \\
1.0931 E+00 \\
1.1269 E+00 \\
1.1133 E+00 \\
1.1027 E+00 \\
1.1230 E+00 \\
1.1363 E+00 \\
1.1092 E+00 \\
1.0622 E+00 \\
1.1241 E+00 \\
1.1621 E+00 \\
1.1924 E+00 \\
1.2508 E+00 \\
1.2991 E+00 \\
1.3586 E+00 \\
1.3990 E+00 \\
1.3910 E+00 \\
1.3974 E+00 \\
1.4235 E+00 \\
1.4317 E+00 \\
1.3347 E+00 \\
1.3976 E+00 \\
1.5557 E+00 \\
1.6399 E+00 \\
1.5517 E+00 \\
1.4987 E+00 \\
1.5240 E+00 \\
1.4249 E+00 \\
1.4443 E+00 \\
1.6190 E+00 \\
1.4557 E+00 \\
1.2846 E+00 \\
1.4933 E+00 \\
6.4775 E-01 \\
1.2219 E+00 \\
1.5104 E+00 \\
1.7676 E+00 \\
2.0925 E+00 \\
2.0259 E+00 \\
6.4708 E+01\end{array}$ \\
\hline
\end{tabular}


TABLE $B-X \times 11$

ZPR-3-11 CENTRAL FLUX SPECTRA

\begin{tabular}{|c|c|c|c|c|}
\hline rROUP & $\begin{array}{r}\text { ENERGY } \\
\text { BOUNDARY } \\
\text { (EV) }\end{array}$ & $\begin{array}{r}1-D \\
\text { DIFFUSION } \\
\text { THEORY } \\
\text { IN/CM2/SI }\end{array}$ & $\begin{array}{r}!-D \\
\text { TRANSPORT } \\
\text { THEORY } \\
\text { IN/CM } / \mathrm{S} \text { ) }\end{array}$ & $\begin{array}{r}2-D \\
\text { DIFFUSION } \\
\text { THEORY } \\
\text { IN/CM2/S }\end{array}$ \\
\hline $\begin{array}{r}1 \\
2 \\
3 \\
4 \\
5 \\
6 \\
7 \\
8 \\
9 \\
10 \\
11 \\
12 \\
13 \\
14 \\
15 \\
16 \\
17 \\
18 \\
19 \\
20 \\
21 \\
22 \\
23 \\
24 \\
25 \\
26 \\
27 \\
28 \\
29 \\
30 \\
31 \\
32 \\
33 \\
34 \\
35 \\
36 \\
37 \\
38 \\
39 \\
40 \\
41 \\
42 \\
43 \\
44 \\
45 \\
46 \\
47 \\
48 \\
49 \\
50\end{array}$ & $\begin{array}{l}1.9971 E+07 \\
1.0000 E+07 \\
6.0653 E+06 \\
3.6788 E+06 \\
2.2313 E+06 \\
1.3534 E+06 \\
8.2085 E+05 \\
4.9787 E+05 \\
3.8774 E+05 \\
3.0197 E+05 \\
2.3518 E+05 \\
1.8316 E+05 \\
1.4264 E+05 \\
1.1109 E+05 \\
8.6517 E+04 \\
6.7379 E+04 \\
5.2475 E+04 \\
4.0868 E+04 \\
3.1828 E+04 \\
2.4788 E+04 \\
1.9305 E+04 \\
1.5034 E+04 \\
1.1709 E+04 \\
9.1188 E+03 \\
7.1017 E+03 \\
5.5308 E+03 \\
4.3074 E+03 \\
3.3546 E+03 \\
2.6126 E+03 \\
2.0347 E+03 \\
1.5846 E+03 \\
1.2341 E+03 \\
9.6112 E+02 \\
7.4852 E+02 \\
5.8295 E+02 \\
4.5400 E+02 \\
3.5358 E+02 \\
2.7536 E+02 \\
1.6702 E+02 \\
1.0130 E+02 \\
6.1442 E+01 \\
3.7267 E+01 \\
2.2603 E+01 \\
1.3710 E+01 \\
8.3153 E+00 \\
5.0435 E+00 \\
3.0590 E+00 \\
1.8554 E+00 \\
1.1254 E+00 \\
6.8256 E-01 \\
1.0000 E-05\end{array}$ & $\begin{array}{l}2.9903 E-02 \\
3.9670 E-01 \\
1.6444 E+00 \\
3.4591 E+00 \\
4.9939 E+00 \\
8.3223 E+00 \\
1.50 / 6 E+01 \\
8.8648 E+00 \\
9.2148 E+00 \\
8.5259 E+00 \\
7.4736 E+00 \\
6.4628 E+00 \\
5.6054 E+00 \\
4.9732 E+00 \\
4.2905 E+00 \\
3.5239 E+00 \\
2.7944 E+00 \\
1.5487 E+00 \\
9.6820 E-01 \\
8.5847 E-01 \\
4.4506 E-01 \\
2.4351 E-01 \\
1.2782 E-01 \\
5.9727 E-02 \\
4.5936 E-02 \\
2.3377 E-02 \\
1.7936 E-02 \\
4.8195 E-03 \\
1.6868 E-03 \\
2.1479 E-03 \\
9.0497 E-04 \\
2.4264 E-04 \\
1.4760 E-04 \\
7.3111 E-05 \\
4.1618 E-05 \\
1.4948 E-05 \\
1.2326 E-05 \\
2.1861 E-05 \\
1.2796 E-05 \\
5.3330 E-07 \\
1.2450 E-08 \\
3.1018 E-10 \\
5.8046 E-12 \\
1.1534 E-13 \\
1.3747 E-15 \\
5.8860 E-17 \\
3.4003 E-18 \\
1.3806 E-19 \\
2.5548 E-21 \\
1.9055 E-23 \\
1.0000 E+02\end{array}$ & $\begin{array}{l}2.9828 E-02 \\
3.9514 E-01 \\
1.6365 E+00 \\
3.4417 E+00 \\
4.9702 E+00 \\
8.2979 E+00 \\
1.5069 E+01 \\
8.8648 E+00 \\
9.2192 E+00 \\
8.5315 E+00 \\
7.4794 E+00 \\
6.4680 E+00 \\
5.6110 E+00 \\
4.9780 E+00 \\
4.2973 E+00 \\
3.5276 E+00 \\
2.8021 E+00 \\
1.5567 E+00 \\
9.7541 E-01 \\
8.5039 E-01 \\
4.4948 E-01 \\
2.4581 E-01 \\
1.2889 E-01 \\
6.0134 E-02 \\
4.6296 E-02 \\
2.3571 E-02 \\
1.8071 E-02 \\
4.8598 E-03 \\
1.6916 E-03 \\
2.1685 E-03 \\
9.1429 E-04 \\
2.4421 E-04 \\
1.4821 E-04 \\
7.3078 E-05 \\
4.1486 E-05 \\
1.4880 E-05 \\
1.2272 E-05 \\
2.1832 E-05 \\
1.2747 E-05 \\
5.3345 E-07 \\
1.2469 E-08 \\
3.1113 E-10 \\
5.8321 E-12 \\
1.1621 E-13 \\
1.3858 E-15 \\
5.9822 E-17 \\
3.4928 E-18 \\
1.4327 E-19 \\
2.6593 E-21 \\
1.9839 E-23 \\
1.0000 E+02\end{array}$ & $\begin{array}{l}2.9904 E-02 \\
3.9673 E-01 \\
1.6446 E+00 \\
3.4595 E+00 \\
4.9940 E+00 \\
8.3208 E+00 \\
1.5071 E+01 \\
8.8619 E+00 \\
9.2121 E+00 \\
8.5239 E+00 \\
7.4723 E+00 \\
6.4623 E+00 \\
5.6057 E+00 \\
4.9742 E+00 \\
4.2922 E+00 \\
3.5259 E+00 \\
2.7969 E+00 \\
1.5506 E+00 \\
9.6957 E-01 \\
8.6015 E-01 \\
4.4611 E-01 \\
2.4411 E-01 \\
1.2810 E-01 \\
5.9845 E-02 \\
4.6035 E-02 \\
2.3429 E-02 \\
1.7971 E-02 \\
4.8294 E-03 \\
1.6900 E-03 \\
2.1523 E-03 \\
9.0691 E-04 \\
2.4303 E-04 \\
1.4776 E-04 \\
7.3149 E-05 \\
4.1631 E-05 \\
1.4951 E-05 \\
1.2328 E-05 \\
2.1865 E-05 \\
1.2798 E-05 \\
5.3341 E-07 \\
1.2453 E-08 \\
3.1025 E-10 \\
5.8060 E-12 \\
1.1537 E-13 \\
1.3751 E-15 \\
5.8906 E-17 \\
3.4313 E-18 \\
1.4106 E-19 \\
2.6245 E-21 \\
1.9587 E-23 \\
1.0000 E+02\end{array}$ \\
\hline
\end{tabular}


TABLE $B-X \times 1 \| 1$

ZPR-3-11 EDGE FLUX SPECTRA

\begin{tabular}{|c|c|c|c|c|}
\hline SROUP & $\begin{array}{r}\text { ENERGY } \\
\text { BOUNDRRY } \\
\text { (EV) }\end{array}$ & $\begin{array}{r}1-0 \\
\text { DIFFUSION } \\
\text { THEORY } \\
\text { IN/CM2/SI }\end{array}$ & $\begin{array}{r}1-0 \\
\text { TRANSPORT } \\
\text { THEORY } \\
\text { [N/CM2/S ] }\end{array}$ & $\begin{array}{r}2-D \\
\text { DIFFUSION } \\
\text { THEORY } \\
(N / C M 2 / S)\end{array}$ \\
\hline $\begin{array}{l}1 \\
2 \\
3 \\
4 \\
5 \\
6 \\
7 \\
8 \\
9 \\
10 \\
11 \\
12 \\
13 \\
14 \\
15 \\
16 \\
17 \\
18 \\
19 \\
20 \\
21 \\
22 \\
23 \\
24 \\
25 \\
26 \\
27 \\
28 \\
29 \\
30 \\
31 \\
32 \\
33 \\
34 \\
35 \\
36 \\
37 \\
38 \\
39 \\
40 \\
41 \\
42 \\
43 \\
44 \\
45 \\
46 \\
47 \\
48 \\
49 \\
50\end{array}$ & $\begin{array}{l}1.9971 E+07 \\
1.0000 E+07 \\
6.0653 E+06 \\
3.6788 E+06 \\
2.2313 E+06 \\
1.3534 E+06 \\
8.2085 E+05 \\
4.97 E 7 E+05 \\
3.8774 E+05 \\
3.0197 E+05 \\
2.3518 E+05 \\
1.8316 E+05 \\
1.4264 E+05 \\
1.1109 E+05 \\
8.6517 E+04 \\
6.7379 E+04 \\
5.2475 E+04 \\
4.0868 E+04 \\
3.1828 E+04 \\
2.4788 E+04 \\
1.9305 E+04 \\
1.5034 E+04 \\
1.1709 E+04 \\
9.1188 E+03 \\
7.1017 E+03 \\
5.5308 E+03 \\
4.3074 E+03 \\
3.3546 E+03 \\
2.6126 E+03 \\
2.0347 E+03 \\
1.5846 E+03 \\
1.2341 E+03 \\
9.6112 E+02 \\
7.4852 E+02 \\
5.8295 E+02 \\
4.5400 E+02 \\
3.5358 E+02 \\
2.7536 E+02 \\
1.6702 E+02 \\
1.0130 E+02 \\
6.1442 E+01 \\
3.7267 E+01 \\
2.2603 E+01 \\
1.3710 E+01 \\
8.3153 E+00 \\
5.0435 E+00 \\
3.0590 E+00 \\
1.8554 E+00 \\
1.1254 E+00 \\
6.8256 E-01 \\
1.0000 E-05\end{array}$ & $\begin{array}{l}3.6140 E-03 \\
3.9601 E-02 \\
1.4969 E-01 \\
3.0606 E-01 \\
4.9497 E-01 \\
1.6399 E+00 \\
8.2809 E+00 \\
5.9977 E+00 \\
7.5441 E+00 \\
8.0459 E+00 \\
7.7607 E+00 \\
7.4130 E+00 \\
7.2766 E+00 \\
7.2168 E+00 \\
7.5975 E+00 \\
6.9539 E+00 \\
6.8155 E+00 \\
4.9215 E+00 \\
3.0685 E+00 \\
3.8025 E+00 \\
1.9154 E+00 \\
1.1983 E+00 \\
6.7512 E-01 \\
3.1329 E-01 \\
2.3141 E-01 \\
1.2390 E-01 \\
1.1708 E-01 \\
5.0552 E-02 \\
2.2205 E-02 \\
1.6267 E-02 \\
5.0710 E-03 \\
1.3872 E-03 \\
6.3241 E-04 \\
1.9569 E-04 \\
8.8131 E-05 \\
3.1478 E-05 \\
1.4633 E-05 \\
2.6198 E-05 \\
6.1316 E-06 \\
1.0606 E-06 \\
1.7337 E-07 \\
1.6894 E-08 \\
7.8218 E-10 \\
1.0931 E-10 \\
1.7595 E-12 \\
2.4281 E-13 \\
4.6720 E-14 \\
8.6062 E-15 \\
1.1831 E-15 \\
9.1385 E-17 \\
1.0000 E+02\end{array}$ & $\begin{array}{l}4.9115 E-03 \\
5.2161 E-02 \\
1.9068 E-01 \\
3.7722 E-01 \\
6.0044 E-01 \\
1.8628 E+00 \\
8.3976 E+00 \\
6.0129 E+00 \\
7.5066 E+00 \\
7.9946 E+00 \\
7.7229 E+00 \\
7.3901 E+00 \\
7.2627 E+00 \\
7.2309 E+00 \\
7.6147 E+00 \\
7.0005 E+00 \\
6.7698 E+00 \\
4.8043 E+00 \\
2.9883 E+00 \\
3.6754 E+00 \\
1.8607 E+00 \\
1.1661 E+00 \\
6.5728 E-01 \\
3.0803 E-01 \\
2.2694 E-01 \\
1.2126 E-01 \\
1.1261 E-01 \\
4.9019 E-02 \\
1.9937 E-02 \\
1.1860 E-02 \\
4.4919 E-03 \\
1.2776 E-03 \\
5.8067 E-04 \\
1.8651 E-04 \\
8.6980 E-05 \\
3.2204 E-05 \\
1.4645 E-05 \\
1.6326 E-05 \\
5.1784 E-06 \\
7.7129 E-07 \\
1.4160 E-07 \\
1.3716 E-08 \\
6.3496 E-10 \\
9.7257 E-11 \\
1.5215 E-12 \\
2.2303 E-13 \\
4.4227 E-14 \\
8.3017 E-15 \\
1.1535 E-15 \\
8.9709 E-17 \\
1.0000 E+02\end{array}$ & $\begin{array}{l}2.9877 E-03 \\
3.3243 E-02 \\
1.2685 E-01 \\
2.5946 E-01 \\
4.1416 E-01 \\
1.3468 E+00 \\
7.3811 E+00 \\
5.4704 E+00 \\
7.0315 E+00 \\
7.6477 E+00 \\
7.5114 E+00 \\
7.2969 E+00 \\
7.2795 E+00 \\
7.3392 E+00 \\
7.8640 E+00 \\
7.3191 E+00 \\
7.2955 E+00 \\
5.3601 E+00 \\
3.3873 E+00 \\
4.2673 E+00 \\
2.1822 E+00 \\
1.3766 E+00 \\
7.7970 E-01 \\
3.6292 E-01 \\
2.6912 E-01 \\
1.4442 E-01 \\
1.3526 E-01 \\
5.9527 E-02 \\
2.6555 E-02 \\
1.9818 E-02 \\
6.4095 E-03 \\
1.7802 E-03 \\
8.1284 E-04 \\
2.5199 E-04 \\
1.1144 E-04 \\
3.9217 E-05 \\
1.7624 E-05 \\
2.8506 E-05 \\
6.3477 E-06 \\
1.0549 E-06 \\
1.8107 E-07 \\
1.7749 E-08 \\
8.3253 E-10 \\
1.2229 E-10 \\
1.9877 E-12 \\
2.8416 E-13 \\
5.8006 E-14 \\
1.1251 E-14 \\
1.6045 E-15 \\
1.2603 E-16 \\
1.0000 E+02\end{array}$ \\
\hline
\end{tabular}


TABLE B-XXIV

ZPR-3-11 CENTRAL ADJOINT SPECTRA

\begin{tabular}{|c|c|c|c|c|}
\hline GROUP & $\begin{array}{r}\text { ENERGY } \\
\text { BOUNDRRY } \\
\text { [EV] }\end{array}$ & $\begin{array}{r}1-D \\
\text { DIFFUSION } \\
\text { THEORY } \\
\text { (RHO/N/S) }\end{array}$ & $\begin{array}{r}1-D \\
\text { TRANSPORT } \\
\text { THEORY } \\
\text { (RHO/N/S) }\end{array}$ & $\begin{array}{r}2-D \\
\text { DIFFUSION } \\
\text { THEORY } \\
\text { iRHO/N/S I }\end{array}$ \\
\hline $\begin{array}{l}1 \\
2 \\
3 \\
4 \\
5 \\
6 \\
7 \\
8 \\
9 \\
10 \\
11 \\
12 \\
13 \\
14 \\
15 \\
16 \\
17 \\
18 \\
19 \\
20 \\
21 \\
22 \\
23 \\
24 \\
25 \\
26 \\
27 \\
28 \\
29 \\
30 \\
31 \\
32 \\
33 \\
34 \\
35 \\
36 \\
37 \\
38 \\
39 \\
40 \\
41 \\
42 \\
43 \\
44 \\
45 \\
46 \\
47 \\
48 \\
49 \\
50\end{array}$ & 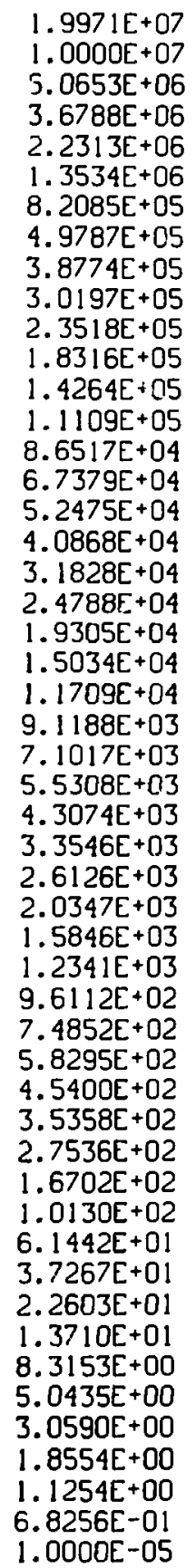 & $\begin{array}{l}2.1818 E+00 \\
1.6240 E+00 \\
1.1884 E+00 \\
1.1563 E+00 \\
1.0281 E+00 \\
8.2166 E-01 \\
7.9257 E-01 \\
8.2765 E-01 \\
8.3531 E-01 \\
8.3967 E-01 \\
8.3855 E-01 \\
8.3543 E-01 \\
8.2804 E-01 \\
8.1403 E-01 \\
8.0267 E-01 \\
7.7383 E-01 \\
7.4440 E-01 \\
7.3905 E-01 \\
7.3411 E-01 \\
7.2349 E-01 \\
7.2851 E-01 \\
7.5182 E-01 \\
7.7475 E-01 \\
8.0287 E-01 \\
8.4532 E-01 \\
8.9632 E-01 \\
9.4281 E-01 \\
9.4378 E-01 \\
9.8361 E-01 \\
1.0447 E+00 \\
1.0627 E+00 \\
9.7457 E-01 \\
1.0933 E+00 \\
1.2437 E+00 \\
1.3337 E+00 \\
1.1633 E+00 \\
1.1166 E+00 \\
1.2647 E+00 \\
1.1686 E+00 \\
1.1298 E+00 \\
1.4802 E+00 \\
1.1855 E+00 \\
9.6441 E-01 \\
1.3605 E+00 \\
3.3008 E-01 \\
9.9649 E-01 \\
1.2532 E+00 \\
1.5685 E+00 \\
1.9425 E+00 \\
1.9085 E+00 \\
5.2384 E+01\end{array}$ & $\begin{array}{l}2.1869 E+00 \\
1.6251 E+00 \\
1.1888 E+00 \\
1.1562 E+00 \\
1.0278 E+00 \\
8.2239 E-01 \\
7.9335 E-01 \\
8.2659 E-01 \\
8.3361 E-01 \\
8.3743 E-01 \\
8.3609 E-01 \\
8.3291 E-01 \\
8.2564 E-01 \\
8.1177 E-01 \\
8.0084 E-01 \\
7.7199 E-01 \\
7.4409 E-01 \\
7.4008 E-01 \\
7.3551 E-01 \\
7.2435 E-01 \\
7.2910 E-01 \\
7.5207 E-01 \\
7.7460 E-01 \\
8.0236 E-01 \\
8.4490 E-01 \\
8.9581 E-01 \\
9.4249 E-01 \\
9.4286 E-01 \\
9.8397 E-01 \\
1.0464 E+00 \\
1.0629 E+00 \\
9.7448 E-01 \\
1.0931 E+00 \\
1.2412 E+00 \\
1.3297 E+00 \\
1.1604 E+00 \\
1.1150 E+00 \\
1.2629 E+00 \\
1.1654 E+00 \\
1.1281 E+00 \\
1.4738 E+00 \\
1.1812 E+00 \\
9.6103 E-01 \\
1.3546 E+00 \\
3.2867 E-01 \\
9.9219 E-01 \\
1.2470 E+00 \\
1.5608 E+00 \\
1.9319 E+00 \\
1.8976 E+00 \\
5.2298 E+01\end{array}$ & $\begin{array}{l}2.1822 E+00 \\
1.6241 E+00 \\
1.1885 E+00 \\
1.1564 E+00 \\
1.0281 E+00 \\
8.2159 E-01 \\
7.9257 E-01 \\
8.2748 E-01 \\
8.3508 E-01 \\
8.3942 E-01 \\
8.3832 E-01 \\
8.3523 E-01 \\
8.2788 E-01 \\
8.1390 E-01 \\
8.0258 E-01 \\
7.7377 E-01 \\
7.4435 E-01 \\
7.3903 E-01 \\
7.3410 E-01 \\
7.2347 E-01 \\
7.2852 E-01 \\
7.5185 E-01 \\
7.7481 E-01 \\
8.0295 E-01 \\
8.4541 E-01 \\
8.9642 E-01 \\
9.4293 E-01 \\
9.4390 E-01 \\
9.8374 E-01 \\
1.0448 E+00 \\
1.0628 E+00 \\
9.7470 E-01 \\
1.0934 E+00 \\
1.2439 E+00 \\
1.3339 E+00 \\
1.1635 E+00 \\
1.1167 E+00 \\
1.2648 E+00 \\
1.1687 E+00 \\
1.1299 E+00 \\
1.4804 E+00 \\
1.1857 E+00 \\
9.6452 E-01 \\
1.3607 E+00 \\
3.3011 E-01 \\
9.9661 E-01 \\
1.2534 E+00 \\
1.5687 E+00 \\
1.9427 E+00 \\
1.9087 E+00 \\
5.2387 E+01\end{array}$ \\
\hline
\end{tabular}


IABLE $B-X X V$

ZPR-3-12 CENTRAL FLUX SPECTRA

\begin{tabular}{|c|c|c|c|c|}
\hline SROUP & $\begin{array}{r}\text { ENERGY } \\
\text { BOUNDRRY } \\
\text { (EV) }\end{array}$ & $\begin{array}{r}\text { DIFFUSION } \\
\text { THEORY } \\
\text { (N/CM2/SI }\end{array}$ & $\begin{array}{r}\text { TRANSPORT } \\
\text { THEORY } \\
\text { (N/CM2/S) }\end{array}$ & $\begin{array}{r}\text { DIFFUSION } \\
\text { THEORY } \\
\text { IN/CM2/SI }\end{array}$ \\
\hline $\begin{array}{r}1 \\
2 \\
3 \\
4 \\
5 \\
6 \\
7 \\
8 \\
9 \\
10 \\
11 \\
12 \\
13 \\
14 \\
15 \\
16 \\
17 \\
18 \\
19 \\
20 \\
21 \\
22 \\
23 \\
24 \\
25 \\
26 \\
27 \\
28 \\
29 \\
30 \\
31 \\
32 \\
33 \\
34 \\
35 \\
36 \\
37 \\
38 \\
39 \\
40 \\
41 \\
42 \\
43 \\
44 \\
45 \\
46 \\
47 \\
48 \\
49 \\
50\end{array}$ & $\begin{array}{l}1.9971 E+07 \\
1.0000 E+07 \\
6.0653 E+06 \\
3.6788 E+06 \\
2.2313 E+06 \\
1.3534 E+06 \\
8.2085 E+05 \\
4.9787 E+05 \\
3.8774 E+05 \\
3.0197 E+05 \\
2.3518 E+05 \\
1.8316 E+05 \\
1.4264 E+05 \\
1.1109 E+05 \\
8.6517 E+04 \\
6.7379 E+04 \\
5.2475 E+04 \\
4.0868 E+04 \\
3.1828 E+04 \\
2.4788 E+04 \\
1.9305 E+04 \\
1.5034 E+04 \\
1.1709 E+04 \\
9.1188 E+03 \\
7.1017 E+03 \\
5.5308 E+03 \\
4.3074 E+03 \\
3.3546 E+03 \\
2.6126 E+03 \\
2.0347 E+03 \\
1.5846 E+03 \\
1.2341 E+03 \\
9.6112 E+02 \\
7.4852 E+02 \\
5.8295 E+02 \\
4.5400 E+02 \\
3.5358 E+02 \\
2.7536 E+02 \\
1.6702 E+02 \\
1.0130 E+02 \\
6.1442 E+01 \\
3.7267 E+01 \\
2.2603 E+01 \\
1.3710 E+01 \\
8.3153 E+00 \\
5.0435 E+00 \\
3.0590 E+00 \\
1.8554 E+00 \\
1.1254 E+00 \\
6.8256 E-01 \\
1.0000 E-05\end{array}$ & $\begin{array}{l}3.6523 E-02 \\
4.5374 E-01 \\
2.1385 E+00 \\
4.8206 E+00 \\
6.9507 E+00 \\
9.3268 E+00 \\
1.2725 E+01 \\
6.8785 E+00 \\
6.9121 E+00 \\
6.5577 E+00 \\
6.0390 E+00 \\
5.5227 E+00 \\
4.9970 E+00 \\
4.4832 E+00 \\
4.0555 E+00 \\
3.5938 E+00 \\
3.1120 E+00 \\
2.4784 E+00 \\
2.0073 E+00 \\
1.8083 E+00 \\
1.3804 E+00 \\
1.0715 E+00 \\
7.8574 E-01 \\
5.5791 E-01 \\
4.1984 E-01 \\
3.0279 E-01 \\
2.1360 E-01 \\
1.4450 E-01 \\
9.3648 E-02 \\
5.8548 E-02 \\
3.5370 E-02 \\
1.8931 E-02 \\
1.0402 E-02 \\
5.1443 E-03 \\
2.4208 E-03 \\
1.1729 E-03 \\
5.4343 E-04 \\
3.0985 E-04 \\
7.0124 E-05 \\
1.3296 E-05 \\
1.6585 E-06 \\
1.9295 E-07 \\
2.1067 E-08 \\
2.2594 E-09 \\
1.8153 E-10 \\
3.7073 E-11 \\
9.5362 E-12 \\
1.7976 E-12 \\
1.5966 E-13 \\
5.1047 E-15 \\
1.0000 E+02\end{array}$ & $\begin{array}{l}3.6719 \mathrm{E}-02 \\
4.5368 \mathrm{E}-01 \\
2.1306 \mathrm{E}+00 \\
4.7931 \mathrm{E}+00 \\
6.9197 \mathrm{E}+00 \\
9.3080 \mathrm{E}+00 \\
1.2726 \mathrm{E}+01 \\
6.8819 \mathrm{E}+00 \\
6.9186 \mathrm{E}+00 \\
6.5652 \mathrm{E}+00 \\
6.0462 \mathrm{E}+00 \\
5.5289 \mathrm{E}+0 \mathrm{C} \\
5.0025 \mathrm{E}+00 \\
4.4872 \mathrm{E}+00 \\
4.0595 \mathrm{E}+00 \\
3.5960 \mathrm{E}+00 \\
3.1139 \mathrm{E}+00 \\
2.4805 \mathrm{E}+00 \\
2.0126 \mathrm{E}+00 \\
1.8137 \mathrm{E}+00 \\
1.3851 \mathrm{E}+00 \\
1.0755 \mathrm{E}+00 \\
7.8901 \mathrm{E}-01 \\
5.6042 \mathrm{E}-01 \\
4.2190 \mathrm{E}-01 \\
3.0442 \mathrm{E}-01 \\
2.1497 \mathrm{E}-01 \\
1.4546 \mathrm{E}-01 \\
9.4395 \mathrm{E}-02 \\
5.9234 \mathrm{E}-02 \\
3.5837 \mathrm{E}-02 \\
1.9214 \mathrm{E}-02 \\
1.0580 \mathrm{E}-02 \\
5.2421 \mathrm{E}-03 \\
2.4706 \mathrm{E}-03 \\
1.1982 \mathrm{E}-03 \\
5.5578 \mathrm{E}-04 \\
3.1789 \mathrm{E}-04 \\
7.1723 \mathrm{E}-05 \\
1.3653 \mathrm{E}-05 \\
1.7056 \mathrm{E}-06 \\
1.9890 \mathrm{E}-07 \\
2.1774 \mathrm{E}-08 \\
2.3395 \mathrm{E}-09 \\
1.8836 \mathrm{E}-10 \\
3.8550 \mathrm{E}-11 \\
9.9306 \mathrm{E}-12 \\
1.8747 \mathrm{E}-12 \\
1.6658 \mathrm{E}-13 \\
5.3277 \mathrm{E}-15 \\
1.0000 \mathrm{E}+02\end{array}$ & $\begin{array}{l}3.6514 \mathrm{E}-02 \\
4.5378 \mathrm{E}-01 \\
2.1385 \mathrm{E}+00 \\
4.8199 \mathrm{E}+00 \\
6.9473 \mathrm{E}+00 \\
9.3188 \mathrm{E}+00 \\
1.2714 \mathrm{E}+01 \\
6.8735 \mathrm{E}+00 \\
6.9079 \mathrm{E}+00 \\
6.5550 \mathrm{E}+00 \\
6.0376 \mathrm{E}+00 \\
5.5226 \mathrm{E}+00 \\
4.9980 \mathrm{E}+00 \\
4.4851 \mathrm{E}+00 \\
4.0581 \mathrm{E}+00 \\
3.5971 \mathrm{E}+00 \\
3.1157 \mathrm{E}+00 \\
2.4822 \mathrm{E}+00 \\
2.0109 \mathrm{E}+00 \\
1.8122 \mathrm{E}+00 \\
1.3837 \mathrm{E}+00 \\
1.0741 \mathrm{E}+00 \\
7.8775 \mathrm{E}-01 \\
5.5933 \mathrm{E}-01 \\
4.2087 \mathrm{E}-01 \\
3.0349 \mathrm{E}-01 \\
2.1404 \mathrm{E}-01 \\
1.4476 \mathrm{E}-01 \\
9.3785 \mathrm{E}-02 \\
5.8611 \mathrm{E}-02 \\
3.5393 \mathrm{E}-02 \\
1.8936 \mathrm{E}-02 \\
1.0400 \mathrm{E}-02 \\
5.1411 \mathrm{E}-03 \\
2.4183 \mathrm{E}-03 \\
1.1712 \mathrm{E}-03 \\
5.4251 \mathrm{E}-04 \\
3.0923 \mathrm{E}-04 \\
6.9993 \mathrm{E}-05 \\
1.3267 \mathrm{E}-05 \\
1.6546 \mathrm{E}-06 \\
1.9246 \mathrm{E}-07 \\
2.1009 \mathrm{E}-08 \\
2.2530 \mathrm{E}-09 \\
1.8099 \mathrm{E}-10 \\
3.6951 \mathrm{E}-11 \\
9.5007 \mathrm{E}-12 \\
1.7904 \mathrm{E}-12 \\
1.5900 \mathrm{E}-13 \\
5.0834 \mathrm{E}-15 \\
1.0000 \mathrm{E}+02\end{array}$ \\
\hline
\end{tabular}


TABLE $B-X X V I$

ZPR-3-12 EOGE FLUX SPECTRA

\begin{tabular}{|c|c|c|c|c|}
\hline GROUP & $\begin{array}{r}\text { ENERGY } \\
\text { BOUNDARY } \\
\text { (EV) }\end{array}$ & $\begin{array}{r}1-D \\
\text { DIFFUSIDN } \\
\text { THEORY } \\
(\mathrm{N} / \mathrm{CM} 2 / \mathrm{S})\end{array}$ & $\begin{array}{r}1-0 \\
\text { TRANSPORT } \\
\text { THEORY } \\
\text { (N/CM2/S) }\end{array}$ & $\begin{array}{r}2-0 \\
\text { DIFFUSION } \\
\text { THEORY } \\
\text { [N/CM2/S }\end{array}$ \\
\hline $\begin{array}{l}1 \\
2 \\
3 \\
4 \\
5 \\
6 \\
7 \\
8 \\
9 \\
10 \\
11 \\
12 \\
13 \\
14 \\
15 \\
16 \\
17 \\
18 \\
19 \\
20 \\
21 \\
22 \\
23 \\
24 \\
25 \\
26 \\
27 \\
28 \\
29 \\
30 \\
31 \\
32 \\
33 \\
34 \\
35 \\
36 \\
37 \\
38 \\
39 \\
40 \\
41 \\
42 \\
43 \\
44 \\
45 \\
46 \\
47 \\
48 \\
49 \\
50\end{array}$ & $\begin{array}{l}1.9971 E+07 \\
1.0000 E+07 \\
6.0653 E+06 \\
3.6788 E+06 \\
2.2313 E+06 \\
1.3534 E+06 \\
8.2085 E+05 \\
4.9787 E+05 \\
3.8774 E+05 \\
3.0197 E+05 \\
2.3518 E+05 \\
1.8316 E+05 \\
1.4264 E+05 \\
1.1109 E+05 \\
8.6517 E+04 \\
6.7379 E+04 \\
5.2475 E+04 \\
4.0868 E+04 \\
3.1828 E+04 \\
2.4788 E+04 \\
1.9305 E+04 \\
1.5034 E+04 \\
1.1709 E+04 \\
9.1188 E+03 \\
7.1017 E+03 \\
5.5308 E+03 \\
4.3074 E+03 \\
3.3546 E+03 \\
2.6126 E+03 \\
2.0347 E+03 \\
1.5846 E+03 \\
1.2341 E+03 \\
9.6112 E+02 \\
7.4852 E+02 \\
5.8295 E+02 \\
4.5400 E+02 \\
3.5358 E+02 \\
2.7536 E+02 \\
1.6702 E+02 \\
1.0130 E+02 \\
6.1442 E+01 \\
3.7267 E+01 \\
2.2603 E+01 \\
1.3710 E+01 \\
8.3153 E+00 \\
5.0435 E+00 \\
3.0590 E+00 \\
1.8554 E+00 \\
1.1254 E+00 \\
6.8256 E-01 \\
1.0000 E-05\end{array}$ & $\begin{array}{l}4.3381 \mathrm{E}-03 \\
4.6568 \mathrm{E}-02 \\
1.7686 \mathrm{E}-01 \\
3.6150 \mathrm{E}-01 \\
5.9234 \mathrm{E}-01 \\
1.9946 \mathrm{I}+00 \\
9.0371 \mathrm{E}+00 \\
6.3525 \mathrm{E}+00 \\
7.8020 \mathrm{E}+00 \\
8.1460 \mathrm{E}+00 \\
7.7102 \mathrm{E}+00 \\
7.2850 \mathrm{E}+00 \\
7.0887 \mathrm{E}+00 \\
6.9293 \mathrm{E}+00 \\
7.2317 \mathrm{E}+00 \\
6.6180 \mathrm{E}+00 \\
6.4008 \mathrm{E}+00 \\
4.6520 \mathrm{E}+00 \\
2.9179 \mathrm{E}+00 \\
3.7206 \mathrm{E}+00 \\
1.9048 \mathrm{E}+00 \\
1.2356 \mathrm{E}+00 \\
7.2437 \mathrm{E}-01 \\
3.4606 \mathrm{E}-01 \\
2.5665 \mathrm{E}-01 \\
1.5401 \mathrm{E}-01 \\
1.3001 \mathrm{E}-01 \\
6.4323 \mathrm{E}-02 \\
3.9806 \mathrm{E}-02 \\
4.4218 \mathrm{E}-02 \\
1.7240 \mathrm{E}-02 \\
6.1873 \mathrm{E}-03 \\
4.1280 \mathrm{E}-03 \\
1.6508 \mathrm{E}-03 \\
9.0772 \mathrm{E}-04 \\
4.0874 \mathrm{E}-04 \\
2.0478 \mathrm{E}-04 \\
3.0418 \mathrm{E}-04 \\
3.6636 \mathrm{E}-05 \\
6.2991 \mathrm{E}-06 \\
1.0394 \mathrm{E}-06 \\
1.0682 \mathrm{E}-07 \\
5.1177 \mathrm{E}-09 \\
8.4396 \mathrm{E}-10 \\
1.3628 \mathrm{E}-11 \\
2.847 \mathrm{E}-12 \\
1.2793 \mathrm{E}-12 \\
4.8319 \mathrm{E}-13 \\
1.0116 \mathrm{E}-13 \\
8.6057 \mathrm{E}-15 \\
1.0000 \mathrm{E}+02\end{array}$ & $\begin{array}{l}6.2161 \mathrm{E}-03 \\
6.3795 \mathrm{E}-02 \\
2.3743 \mathrm{E}-01 \\
4.6881 \mathrm{E}-01 \\
7.5473 \mathrm{E}-01 \\
2.3076 \mathrm{E}+00 \\
9.1872 \mathrm{E}+00 \\
6.3737 \mathrm{E}+00 \\
7.7595 \mathrm{E}+00 \\
8.0893 \mathrm{E}+00 \\
7.6706 \mathrm{E}+00 \\
7.2606 \mathrm{E}+00 \\
7.0716 \mathrm{E}+00 \\
6.9415 \mathrm{E}+00 \\
7.2411 \mathrm{E}+00 \\
6.6580 \mathrm{E}+00 \\
6.3374 \mathrm{E}+00 \\
4.5132 \mathrm{E}+00 \\
2.8176 \mathrm{E}+00 \\
3.5528 \mathrm{E}+00 \\
1.8269 \mathrm{E}+00 \\
1.1855 \mathrm{E}+00 \\
6.9258 \mathrm{E}-01 \\
3.3434 \mathrm{E}-01 \\
2.4646 \mathrm{E}-01 \\
1.4733 \mathrm{E}-01 \\
1.2120 \mathrm{E}-01 \\
6.0358 \mathrm{E}-02 \\
3.1102 \mathrm{E}-02 \\
2.1437 \mathrm{E}-02 \\
1.0883 \mathrm{E}-02 \\
4.0653 \mathrm{E}-03 \\
2.7420 \mathrm{E}-03 \\
1.1781 \mathrm{E}-03 \\
7.2249 \mathrm{E}-04 \\
3.5800 \mathrm{E}-04 \\
1.7816 \mathrm{E}-04 \\
1.6278 \mathrm{E}-04 \\
2.6592 \mathrm{E}-05 \\
4.2189 \mathrm{E}-06 \\
8.2652 \mathrm{E}-07 \\
8.5039 \mathrm{E}-08 \\
4.0849 \mathrm{E}-09 \\
7.6686 \mathrm{E}-10 \\
1.1896 \mathrm{E}-11 \\
2.8178 \mathrm{E}-12 \\
1.3783 \mathrm{E}-12 \\
5.3124 \mathrm{E}-13 \\
1.1132 \mathrm{E}-13 \\
9.5045 \mathrm{E}-15 \\
1.0000 \mathrm{E}+02\end{array}$ & $\begin{array}{l}4.3521 \mathrm{E}-03 \\
4.6774 \mathrm{E}-02 \\
1.7812 \mathrm{E}-01 \\
3.6552 \mathrm{E}-01 \\
6.0164 \mathrm{E}-01 \\
2.0262 \mathrm{E}+00 \\
9.0366 \mathrm{E}+00 \\
6.3642 \mathrm{E}+00 \\
7.8057 \mathrm{E}+00 \\
8.1518 \mathrm{E}+00 \\
7.728 \mathrm{E}+00 \\
7.3044 \mathrm{E}+00 \\
7.1050 \mathrm{E}+00 \\
6.9543 \mathrm{E}+00 \\
7.2489 \mathrm{E}+00 \\
6.6264 \mathrm{E}+00 \\
6.3852 \mathrm{E}+00 \\
4.6169 \mathrm{E}+00 \\
2.9116 \mathrm{E}+00 \\
3.6653 \mathrm{E}+00 \\
1.8786 \mathrm{E}+00 \\
1.2194 \mathrm{E}+00 \\
7.1479 \mathrm{E}-01 \\
3.4407 \mathrm{E}-01 \\
2.5507 \mathrm{E}-01 \\
1.5325 \mathrm{E}-01 \\
1.2961 \mathrm{E}-01 \\
6.4232 \mathrm{E}-02 \\
3.9420 \mathrm{E}-02 \\
4.3042 \mathrm{E}-02 \\
1.7194 \mathrm{E}-02 \\
6.2147 \mathrm{E}-03 \\
4.1401 \mathrm{E}-03 \\
1.6753 \mathrm{E}-03 \\
9.2514 \mathrm{E}-04 \\
4.2060 \mathrm{E}-04 \\
2.1052 \mathrm{E}-04 \\
2.9891 \mathrm{E}-04 \\
3.7044 \mathrm{E}-05 \\
6.2375 \mathrm{E}-06 \\
1.0546 \mathrm{E}-06 \\
1.0716 \mathrm{E}-07 \\
5.1368 \mathrm{E}-09 \\
8.5441 \mathrm{E}-10 \\
1.3902 \mathrm{E}-11 \\
2.9072 \mathrm{E}-12 \\
1.3343 \mathrm{E}-12 \\
5.0554 \mathrm{E}-13 \\
1.0548 \mathrm{E}-13 \\
9.0284 \mathrm{E}-15 \\
1.0000 \mathrm{E}+02\end{array}$ \\
\hline
\end{tabular}


TABLE $B-X X Y I I$

ZPR-3-12 CENTRAL ADJOINT SPECTRA

\begin{tabular}{|c|c|c|c|c|}
\hline GROUP & $\begin{array}{r}\text { ENERGY } \\
\text { BOUNDARY } \\
\text { (EV) }\end{array}$ & $\begin{array}{r}1-D \\
\text { DIFFUSION } \\
\text { THEORY } \\
\text { IRHO/N/SI }\end{array}$ & $\begin{array}{r}1-D \\
\text { TRANSPORT } \\
\text { THEORY } \\
\text { [RHO/N/S] }\end{array}$ & $\begin{array}{r}2-D \\
\text { DIFFUSION } \\
\text { THEORY } \\
\text { (RHO/N/S) }\end{array}$ \\
\hline $\begin{array}{r}1 \\
2 \\
3 \\
4 \\
5 \\
6 \\
7 \\
8 \\
9 \\
10 \\
11 \\
12 \\
13 \\
14 \\
15 \\
16 \\
17 \\
18 \\
19 \\
20 \\
21 \\
22 \\
23 \\
24 \\
25 \\
26 \\
27 \\
28 \\
29 \\
30 \\
31 \\
32 \\
33 \\
34 \\
35 \\
36 \\
37 \\
38 \\
39 \\
40 \\
41 \\
42 \\
43 \\
44 \\
45 \\
46 \\
47 \\
48 \\
49 \\
50\end{array}$ & $\begin{array}{l}1.9971 E+07 \\
1.0000 E+07 \\
6.0653 E+06 \\
3.6788 E+06 \\
2.2313 E+06 \\
1.3534 E+06 \\
8.2085 E+05 \\
4.9787 E+05 \\
3.8774 E+05 \\
3.0197 E+05 \\
2.3518 E+05 \\
1.8316 E+05 \\
1.4264 E+05 \\
1.1109 E+05 \\
8.6517 E+04 \\
6.7379 E+04 \\
5.2475 E+04 \\
4.0868 E+04 \\
3.1828 E+04 \\
2.4788 E+04 \\
1.9305 E+04 \\
1.5034 E+04 \\
1.1709 E+04 \\
9.1188 E+03 \\
7.1017 E+03 \\
5.5308 E+03 \\
4.3074 E+03 \\
3.3546 E+03 \\
2.6126 E+03 \\
2.0347 E+03 \\
1.5846 E+03 \\
1.2341 E+03 \\
9.6112 E+02 \\
7.4852 E+02 \\
5.8295 E+02 \\
4.5400 E+02 \\
3.5358 E+02 \\
2.7536 E+02 \\
1.6702 E+02 \\
1.0130 E+02 \\
6.1442 E+01 \\
3.7267 E+01 \\
2.2603 E+01 \\
1.3710 E+01 \\
8.3153 E+00 \\
5.0435 E+00 \\
3.0590 E+00 \\
1.8554 E+00 \\
1.1254 E+00 \\
6.8256 E-01 \\
1.0000 E-05\end{array}$ & $\begin{array}{l}1.8015 E+00 \\
1.3893 E+00 \\
1.1222 E+00 \\
1.0994 E+00 \\
9.9748 E-01 \\
8.7077 E-01 \\
8.7335 E-01 \\
9.1537 E-01 \\
9.3073 E-01 \\
9.4472 E-01 \\
9.5384 E-01 \\
9.5976 E-01 \\
9.6147 E-01 \\
9.6169 E-01 \\
9.5850 E-01 \\
9.5173 E-01 \\
9.4795 E-01 \\
9.5439 E-01 \\
9.6267 E-01 \\
9.6695 E-01 \\
9.8793 E-01 \\
1.0120 E+00 \\
1.0374 E+00 \\
1.0680 E+00 \\
1.1048 E+00 \\
1.1398 E+00 \\
1.1675 E+00 \\
1.1762 E+00 \\
1.2000 E+00 \\
1.2286 E+00 \\
1.2383 E+00 \\
1.2256 E+00 \\
1.3069 E+00 \\
1.4085 E+00 \\
1.4542 E+00 \\
1.3980 E+00 \\
1.3772 E+00 \\
1.3900 E+00 \\
1.2915 E+00 \\
1.3220 E+00 \\
1.5263 E+00 \\
1.3006 E+00 \\
1.1235 E+00 \\
1.3491 E+00 \\
4.9684 E-01 \\
1.1892 E+00 \\
1.4705 E+00 \\
1.7481 E+00 \\
2.3374 E+00 \\
1.9932 E+00 \\
5.9293 E+01\end{array}$ & $\begin{array}{l}1.8242 E+00 \\
1.4011 E+00 \\
1.1272 E+00 \\
1.1007 E+00 \\
9.9789 E-01 \\
8.7035 E-01 \\
8.7049 E-01 \\
9.0975 E-01 \\
9.2428 E-01 \\
9.3721 E-01 \\
9.4645 E-01 \\
9.5232 E-01 \\
9.5418 E-01 \\
9.5461 E-01 \\
9.5190 E-01 \\
9.4545 E-01 \\
9.4260 E-01 \\
9.4970 E-01 \\
9.5837 E-01 \\
9.6142 E-01 \\
9.8210 E-01 \\
1.0059 E+00 \\
1.0310 E+00 \\
1.0613 E+00 \\
1.0980 E+00 \\
1.1328 E+00 \\
1.1606 E+00 \\
1.1690 E+00 \\
1.1935 E+00 \\
1.2217 E+00 \\
1.2296 E+00 \\
1.2170 E+00 \\
1.2974 E+00 \\
1.3973 E+00 \\
1.4422 E+00 \\
1.3863 E+00 \\
1.3667 E+00 \\
1.3803 E+00 \\
1.2816 E+00 \\
1.3117 E+00 \\
1.5119 E+00 \\
1.2893 E+00 \\
1.1138 E+00 \\
1.3365 E+00 \\
4.9243 E-01 \\
1.1781 E+00 \\
1.4555 E+00 \\
1.7304 E+00 \\
2.0158 E+00 \\
1.9718 E+00 \\
5.8942 E+01\end{array}$ & $\begin{array}{l}1.8039 E+00 \\
1.3906 E+00 \\
1.1228 E+00 \\
1.0998 E+00 \\
9.9753 E-01 \\
8.7036 E-01 \\
8.7284 E-01 \\
9.1471 E-01 \\
9.3004 E-01 \\
9.4364 E-01 \\
9.5321 E-01 \\
9.5921 E-01 \\
9.6099 E-01 \\
9.6129 E-01 \\
9.5819 E-01 \\
9.5151 E-01 \\
9.4781 E-01 \\
9.5435 E-01 \\
9.6272 E-01 \\
9.6705 E-01 \\
9.8817 E-01 \\
1.0124 E+00 \\
1.0379 E+00 \\
1.0686 E+00 \\
1.1055 E+00 \\
1.1406 E+00 \\
1.1684 E+00 \\
1.1771 E+00 \\
1.2010 E+00 \\
1.2297 E+00 \\
1.2394 E+00 \\
1.2267 E+00 \\
1.3081 E+00 \\
1.4098 E+00 \\
1.4556 E+00 \\
1.3994 E+00 \\
1.3785 E+00 \\
1.3913 E+00 \\
1.2927 E+00 \\
1.3233 E+00 \\
1.5277 E+00 \\
1.3019 E+00 \\
1.1245 E+00 \\
1.3504 E+00 \\
4.9731 E-01 \\
1.1903 E+00 \\
1.4719 E+00 \\
1.7497 E+00 \\
2.0393 E+00 \\
1.9950 E+00 \\
5.9325 E+01\end{array}$ \\
\hline
\end{tabular}


TABLE B-XXVIII

ZEBRA-2 CENTRAL FLUX SPECTRA

\begin{tabular}{|c|c|c|c|c|}
\hline GROUP & $\begin{array}{r}\text { ENERGY } \\
\text { BOUNDARY } \\
\text { (EV) }\end{array}$ & $\begin{array}{r}1-D \\
\text { DIFFUSION } \\
\text { THEORY } \\
\text { (N/CM } 2 / S \text { ) }\end{array}$ & $\begin{array}{r}1-0 \\
\text { TRANSPORT } \\
\text { THEORY } \\
\text { (N/CM2/S) }\end{array}$ & $\begin{array}{r}2-D \\
\text { DIFFUSION } \\
\text { THEORY } \\
\text { (N/CM?/S) }\end{array}$ \\
\hline $\begin{array}{r}1 \\
2 \\
3 \\
4 \\
5 \\
6 \\
7 \\
8 \\
9 \\
10 \\
11 \\
12 \\
13 \\
14 \\
15 \\
16 \\
17 \\
18 \\
19 \\
20 \\
21 \\
22 \\
23 \\
24 \\
25 \\
26 \\
27 \\
28 \\
29 \\
30 \\
31 \\
32 \\
33 \\
34 \\
35 \\
36 \\
37 \\
38 \\
39 \\
40 \\
41 \\
42 \\
43 \\
44 \\
45 \\
46 \\
47 \\
48 \\
49 \\
50\end{array}$ & $\begin{array}{l}1.9971 E+07 \\
1.0000 E+07 \\
6.0653 \mathrm{E}+06 \\
3.6788 \mathrm{0}+06 \\
2.2313 \mathrm{E}+06 \\
1.3534 \mathrm{E}+06 \\
8.2085 \mathrm{E}+05 \\
4.9787 \mathrm{E}+05 \\
3.8774 \mathrm{E}+05 \\
3.0197 \mathrm{E}+05 \\
2.3518 \mathrm{E}+05 \\
1.8316 \mathrm{E}+05 \\
1.4264 \mathrm{E}+05 \\
1.1109 \mathrm{E}+05 \\
8.6517 \mathrm{E}+04 \\
6.7379 \mathrm{E}+04 \\
5.2475 \mathrm{E}+04 \\
4.0868 \mathrm{E}+04 \\
3.1828 \mathrm{E}+04 \\
2.4788 \mathrm{E}+04 \\
1.9305 \mathrm{E}+04 \\
1.5034 \mathrm{E}+04 \\
1.1709 \mathrm{E}+04 \\
9.1188 \mathrm{E}+03 \\
7.1017 \mathrm{E}+03 \\
5.5308 \mathrm{E}+03 \\
4.3074 \mathrm{E}+03 \\
3.3546 \mathrm{E}+03 \\
2.6126 \mathrm{E}+03 \\
2.0347 \mathrm{E}+03 \\
1.5846 \mathrm{E}+03 \\
1.2341 \mathrm{E}+03 \\
9.6112 \mathrm{E}+02 \\
7.4852 \mathrm{E}+02 \\
5.8295 \mathrm{E}+02 \\
4.5400 \mathrm{E}+02 \\
3.5358 \mathrm{E}+02 \\
2.7536 \mathrm{E}+02 \\
1.6702 \mathrm{E}+02 \\
1.0130 \mathrm{E}+02 \\
6.1442 \mathrm{E}+01 \\
3.7267 \mathrm{E}+01 \\
2.2603 \mathrm{E}+01 \\
1.3710 \mathrm{E}+01 \\
8.3153 \mathrm{E}+00 \\
5.0435 \mathrm{E}+00 \\
3.0590 \mathrm{E}+00 \\
1.8554 \mathrm{E}+00 \\
1.1254 \mathrm{E}+00 \\
6.8256 \mathrm{E}-01 \\
1.0000 \mathrm{E}-05\end{array}$ & $\begin{array}{l}2.9143 \mathrm{E}-02 \\
3.4911 \mathrm{E}-01 \\
1.7292 \mathrm{E}+00 \\
3.9474 \mathrm{E}+00 \\
5.8077 \mathrm{E}+00 \\
7.7535 \mathrm{E}+00 \\
1.0219 \mathrm{E}+01 \\
5.5454 \mathrm{E}+00 \\
5.6181 \mathrm{E}+00 \\
5.4706 \mathrm{E}+00 \\
5.2138 \mathrm{E}+00 \\
4.9311 \mathrm{E}+00 \\
4.6435 \mathrm{E}+00 \\
4.3646 \mathrm{E}+00 \\
4.1029 \mathrm{E}+00 \\
3.8245 \mathrm{E}+00 \\
3.5034 \mathrm{E}+00 \\
3.1199 \mathrm{E}+00 \\
2.8256 \mathrm{C}+00 \\
2.6106 \mathrm{E}+00 \\
2.2951 \mathrm{E}+00 \\
2.0221 \mathrm{E}+00 \\
1.7421 \mathrm{E}+00 \\
1.4915 \mathrm{E}+00 \\
1.2938 \mathrm{E}+00 \\
1.1057 \mathrm{E}+00 \\
9.3500 \mathrm{E}-01 \\
7.7731 \mathrm{E}-01 \\
6.3872 \mathrm{E}-01 \\
5.2072 \mathrm{E}-01 \\
4.1436 \mathrm{E}-01 \\
3.1474 \mathrm{E}-01 \\
2.4030 \mathrm{E}-01 \\
1.7573 \mathrm{E}-01 \\
1.2609 \mathrm{E}-01 \\
9.0673 \mathrm{E}-02 \\
6.3746 \mathrm{E}-02 \\
6.8734 \mathrm{E}-02 \\
2.8247 \mathrm{E}-02 \\
1.1456 \mathrm{E}-02 \\
3.5912 \mathrm{E}-03 \\
1.0001 \mathrm{E}-03 \\
2.5541 \mathrm{E}-04 \\
6.8704 \mathrm{E}-05 \\
1.3061 \mathrm{E}-05 \\
5.6949 \mathrm{E}-06 \\
2.9969 \mathrm{E}-06 \\
1.2900 \mathrm{E}-06 \\
3.2734 \mathrm{E}-07 \\
2.7548 \mathrm{E}-08 \\
1.0000 \mathrm{E}+02\end{array}$ & $\begin{array}{l}2.9165 E-02 \\
3.4899 E-01 \\
1.7263 E+00 \\
3.9381 E+00 \\
5.7949 E+00 \\
7.7398 E+00 \\
1.0236 E+01 \\
5.5394 E+00 \\
5 . E 130 E+00 \\
5.4564 E+00 \\
5.2206 E+00 \\
4.9288 E+00 \\
4.6419 E+00 \\
4.3637 E+00 \\
4.1028 E+00 \\
3.8250 E+00 \\
3.5048 E+00 \\
3.1221 E+00 \\
2.8295 E+00 \\
2.6153 E+00 \\
2.3001 E+00 \\
2.0275 E+00 \\
1.7475 E+00 \\
1.4968 E+00 \\
1.2990 E+00 \\
1.1107 E+00 \\
9.3978 E-01 \\
7.8183 E-01 \\
6.4304 E-01 \\
5.2436 E-01 \\
4.1749 E-01 \\
3.1731 E-01 \\
2.4239 E-01 \\
1.7734 E-01 \\
1.2730 E-01 \\
9.1572 E-02 \\
6.4423 E-02 \\
6.9532 E-02 \\
2.8605 E-02 \\
1.1615 E-02 \\
3.6433 E-03 \\
1.0156 E-03 \\
2.5964 E-04 \\
6.9888 E-05 \\
1.3299 E-05 \\
5.8005 E-06 \\
3.0529 E-06 \\
1.3144 E-06 \\
3.3358 E-07 \\
2.8080 E-08 \\
1.0000 E+02\end{array}$ & $\begin{array}{l}2.9149 E-02 \\
3.4921 E-01 \\
1.7296 E+00 \\
3.9484 E+00 \\
5.8088 E+00 \\
7.7541 E+00 \\
1.0249 E+01 \\
5.5451 E+00 \\
5.6176 E+00 \\
5.4700 E+00 \\
5.2131 E+00 \\
4.9304 E+00 \\
4.6428 E+00 \\
4.3640 E+00 \\
4.1023 E+00 \\
3.8240 E+00 \\
3.5030 E+00 \\
3.1194 E+00 \\
2.8253 E+00 \\
2.6105 E+00 \\
2.2951 E+00 \\
2.0222 E+00 \\
1.7423 E+00 \\
1.4917 E+00 \\
1.2941 E+00 \\
1.1060 E+00 \\
9.3531 E-01 \\
7.7762 E-01 \\
6.3901 E-01 \\
5.2099 E-01 \\
4.1460 E-01 \\
3.1494 E-01 \\
2.4046 E-01 \\
1.7585 E-01 \\
1.2618 E-01 \\
9.0740 E-02 \\
6.3794 E-02 \\
6.8784 E-02 \\
2.8266 E-02 \\
1.1463 E-02 \\
3.5934 E-03 \\
1.0007 E-03 \\
2.5554 E-04 \\
6.8735 E-05 \\
1.3066 E-05 \\
5.6964 E-06 \\
2.9971 E-06 \\
1.2898 E-06 \\
3.2727 E-07 \\
2.7542 E-08 \\
1.0000 E+02\end{array}$ \\
\hline
\end{tabular}




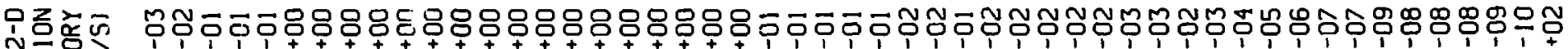

N

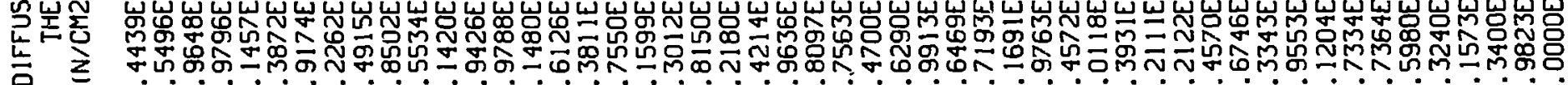

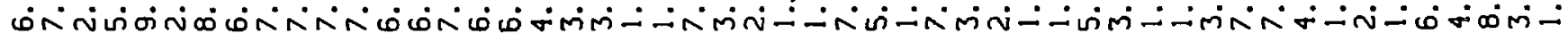

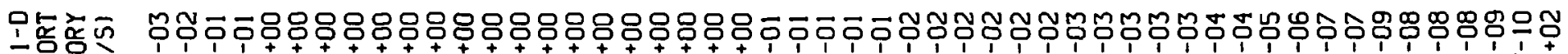

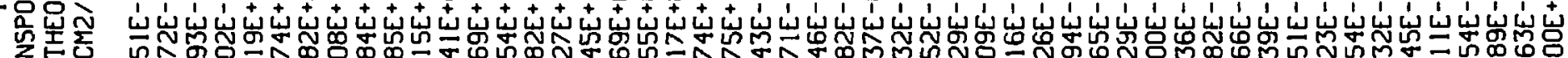
蛋 之

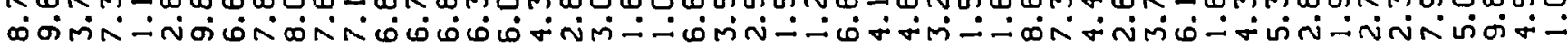

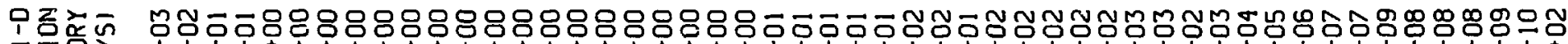

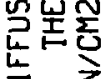

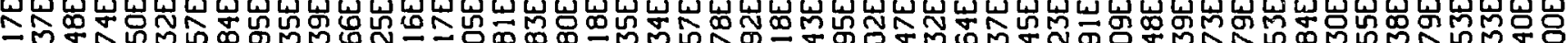

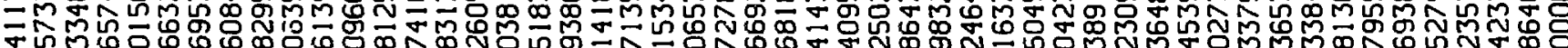

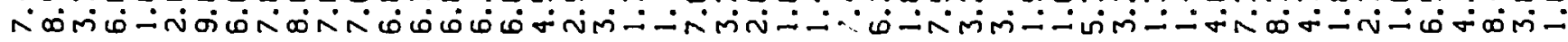

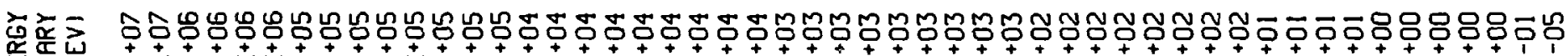

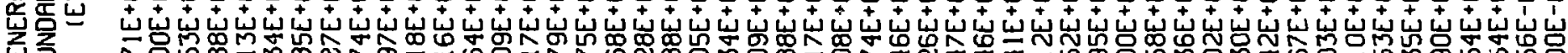

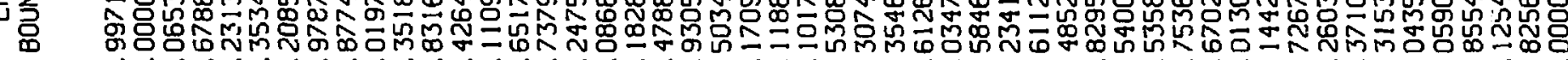

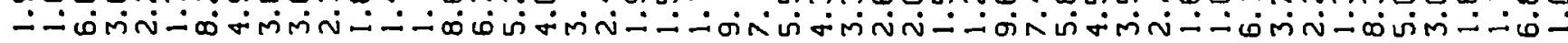


TABLE $B-X X X$

ZEBRA-2 CENTRAL RDJOINT SPECTRA

\begin{tabular}{|c|c|c|c|c|}
\hline GROUP & $\begin{array}{r}\text { ENERGY } \\
\text { BOUNDARY } \\
\text { (EV) }\end{array}$ & $\begin{array}{r}1-0 \\
\text { DIFFUSION } \\
\text { THEDRY } \\
(R H O / N / S)\end{array}$ & $\begin{array}{r}1-0 \\
\text { TRANSPORT } \\
\text { THEORY } \\
\text { (RHO/N/S) }\end{array}$ & $\begin{array}{r}2-0 \\
\text { DIFFUSION } \\
\text { THEORY } \\
\text { (RHO/N/S) }\end{array}$ \\
\hline $\begin{array}{l}1 \\
2 \\
3 \\
4 \\
5 \\
6 \\
7 \\
8 \\
9 \\
10 \\
11 \\
12 \\
13 \\
14 \\
15 \\
16 \\
17 \\
18 \\
19 \\
20 \\
21 \\
22 \\
23 \\
24 \\
25 \\
26 \\
27 \\
28 \\
29 \\
30 \\
31 \\
32 \\
33 \\
34 \\
35 \\
36 \\
37 \\
38 \\
39 \\
40 \\
41 \\
42 \\
43 \\
44 \\
45 \\
46 \\
47 \\
48 \\
49 \\
50\end{array}$ & $\begin{array}{l}1.9971 E+07 \\
1.0000 E+07 \\
6.0653 E+06 \\
3.6788 E+06 \\
2.2313 E+06 \\
1.3534 E+06 \\
8.2085 E+05 \\
4.9787 E+05 \\
3.8774 E+05 \\
3.0197 E+05 \\
2.3518 E+05 \\
1.8315 E+05 \\
1.4264 E+05 \\
1.1109 E+05 \\
8.6517 E+04 \\
6.7379 E+04 \\
5.2475 E+04 \\
4.0868 E+04 \\
3.1828 E+04 \\
2.4788 E+04 \\
1.9305 E+04 \\
1.5034 E+04 \\
1.1709 E+04 \\
9.1188 E+03 \\
7.1017 E+03 \\
5.5308 E+03 \\
4.3074 E+03 \\
3.3546 E+03 \\
2.6126 E+03 \\
2.0347 E+03 \\
1.5846 E+03 \\
1.2341 E+03 \\
9.6112 E+02 \\
7.4852 E+02 \\
5.8295 E+02 \\
4.5400 E+02 \\
3.5358 E+02 \\
2.7536 E+02 \\
1.6702 E+02 \\
1.0130 E+02 \\
6.1442 E E+01 \\
3.7267 E+01 \\
2.2603 E+01 \\
1.3710 E+01 \\
8.3153 E+00 \\
5.0435 E+00 \\
3.0590 E+00 \\
1.8554 E+00 \\
1.1254 E+00 \\
6.8256 E-01 \\
1.0000 E-05\end{array}$ & $\begin{array}{l}1.8050 E+00 \\
1.3990 E+00 \\
1.1577 E+00 \\
1.1159 E+00 \\
9.9754 E-01 \\
8.6781 E-01 \\
8.6822 E-01 \\
8.8614 E-01 \\
8.9011 E-01 \\
8.9233 E-01 \\
8.9287 E-01 \\
8.9288 E-01 \\
8.9154 E-01 \\
8.8987 E-01 \\
8.8785 E-01 \\
8.8540 E-01 \\
8.8684 E-01 \\
8.9482 E-01 \\
9.0474 E-01 \\
9.1540 E-01 \\
9.3245 E-01 \\
9.5245 E-01 \\
9.7385 E-01 \\
9.9767 E-01 \\
1.0251 E+00 \\
1.0521 E+00 \\
1.0767 E+00 \\
1.0937 E+00 \\
1.1203 E+00 \\
1.1485 E+00 \\
1.1683 E+00 \\
1.1812 E+00 \\
1.2390 E+00 \\
1.2931 E+00 \\
1.3143 E+00 \\
1.2852 E+00 \\
1.2770 E+00 \\
1.2838 E+00 \\
1.2193 E+00 \\
1.2693 E+00 \\
1.4175 E+00 \\
1.1853 E+00 \\
1.0338 E+00 \\
1.1831 E+00 \\
5.3851 E-01 \\
1.2837 E+00 \\
1.5596 E+00 \\
1.7965 E+00 \\
2.0069 E+00 \\
1.9833 E+00 \\
5.6713 E+01\end{array}$ & $\begin{array}{l}1.8138 E+00 \\
1.4039 E+00 \\
1.1596 E+00 \\
1.1162 E+00 \\
9.9737 E-01 \\
8.6749 E-01 \\
8.6718 E-01 \\
8.8455 E-01 \\
8.8844 E-01 \\
8.9064 E-01 \\
8.9123 E-01 \\
8.9132 E-01 \\
8.9009 E-01 \\
8.8854 E-01 \\
8.8668 E-01 \\
8.8437 E-01 \\
8.8606 E-01 \\
8.9419 E-01 \\
9.0417 E-01 \\
9.1457 E-01 \\
9.3156 E-01 \\
9.5148 E-01 \\
9.7280 E-01 \\
9.9653 E-01 \\
1.0239 E+00 \\
1.0508 E+00 \\
1.0753 E+00 \\
1.0921 E+00 \\
1.1188 E+00 \\
1.1467 E+00 \\
1.1658 E+00 \\
1.1785 E+00 \\
1.2358 E+00 \\
1.2894 E+00 \\
1.3102 E+00 \\
1.2814 E+00 \\
1.2735 E+00 \\
1.2805 E+00 \\
1.2156 E+00 \\
1.2651 E+00 \\
1.4117 E+00 \\
1.1809 E+00 \\
1.0300 E+00 \\
1.1782 E+00 \\
5.3649 E-01 \\
1.2781 E+00 \\
1.5524 E+00 \\
1.7881 E+00 \\
1.9971 E+00 \\
1.9733 E+00 \\
5.6602 E+01\end{array}$ & $\begin{array}{l}1.8057 E+00 \\
1.3993 E+00 \\
1.1578 E+00 \\
1.1160 E+00 \\
9.9750 E-01 \\
8.6766 E-01 \\
8.6806 E-01 \\
8.8606 E-01 \\
8.9009 E-01 \\
8.9237 E-01 \\
8.9296 E-01 \\
8.9302 E-01 \\
8.9171 E-01 \\
8.9007 E-01 \\
8.8807 E-01 \\
8.8564 E-01 \\
8.8710 E-01 \\
8.9510 E-01 \\
9.0504 E-01 \\
9.1570 E-01 \\
9.3276 E-01 \\
9.5277 E-01 \\
9.7418 E-01 \\
9.9801 E-01 \\
1.0255 E+00 \\
1.0525 E+00 \\
1.0771 E+00 \\
1.0940 E+00 \\
1.1207 E+00 \\
1.1489 E+00 \\
1.1687 E+00 \\
1.1816 E+00 \\
1.2394 E+00 \\
1.2936 E+00 \\
1.3147 E+00 \\
1.2856 E+00 \\
1.2774 E+00 \\
1.2842 E+00 \\
1.2197 E+00 \\
1.2697 E+00 \\
1.4179 E+00 \\
1.1857 E+00 \\
1.0341 E+00 \\
1.1835 E+00 \\
5.3868 E-01 \\
1.2841 E+00 \\
1.5601 E+00 \\
1.7971 E+00 \\
2.0076 E+00 \\
1.9839 E+00 \\
5.6729 E+01\end{array}$ \\
\hline
\end{tabular}


TABLE $8-x \times \times 1$

ZPPR-2 CENTRAL FLUX SPECTRA

\begin{tabular}{|c|c|c|c|c|}
\hline GROUP & $\begin{array}{r}\text { ENERGY } \\
\text { BOUNDARY } \\
\text { (EV) }\end{array}$ & $\begin{array}{r}\text { I-D } \\
\text { OIFFUSION } \\
\text { THEORY } \\
\text { (N/CM2/S) }\end{array}$ & $\begin{array}{r}1-0 \\
\text { TRANSPORT } \\
\text { THEORY } \\
\text { IN/CM } 2 / S \text { I }\end{array}$ & $\begin{array}{r}2-D \\
\text { DIFFUSION } \\
\text { THEORY } \\
\text { IN/CMZZ/SI }\end{array}$ \\
\hline $\begin{array}{l}1 \\
2 \\
3 \\
4 \\
5 \\
6 \\
7 \\
8 \\
9 \\
10 \\
11 \\
12 \\
13 \\
14 \\
15 \\
16 \\
17 \\
18 \\
19 \\
20 \\
21 \\
22 \\
23 \\
24 \\
25 \\
26 \\
27 \\
28 \\
29 \\
30 \\
31 \\
32 \\
33 \\
34 \\
35 \\
36 \\
37 \\
38 \\
39 \\
40 \\
41 \\
42 \\
43 \\
44 \\
45 \\
46 \\
47 \\
48 \\
49 \\
50\end{array}$ & $\begin{array}{l}1.9971 E+07 \\
1.0000 E+07 \\
6.0653 E+06 \\
3.6788 E+06 \\
2.2313 E+06 \\
1.3534 E+06 \\
8.2085 E+05 \\
4.9787 E+05 \\
3.8774 E+05 \\
3.0197 E+05 \\
2.3518 E+05 \\
1.8316 E+05 \\
1.4264 E+05 \\
1.1109 E+05 \\
8.6517 E+04 \\
6.7379 E+04 \\
5.2475 E+04 \\
4.0868 E+04 \\
3.1828 E+04 \\
2.4788 E+04 \\
1.9305 E+04 \\
1.5034 E+04 \\
1.1709 E+04 \\
9.1188 E+03 \\
7.1017 E+03 \\
5.5308 E+03 \\
4.3074 E+03 \\
3.3546 E+03 \\
2.6126 E+03 \\
2.0347 E+03 \\
1.5846 E+03 \\
1.2341 E+03 \\
9.6112 E+02 \\
7.4852 E+02 \\
5.8295 E+02 \\
4.5400 E+02 \\
3.5358 E+02 \\
2.7536 E+02 \\
1.6702 E+02 \\
1.0130 E+02 \\
6.1442 E+01 \\
3.7267 E+01 \\
2.2603 E+01 \\
1.3710 E+01 \\
8.3153 E+00 \\
5.0435 E+00 \\
3.0590 E+00 \\
1.8554 E+00 \\
1.1254 E+00 \\
6.8256 E-01 \\
1.0000 E-05\end{array}$ & $\begin{array}{l}2.2513 E-02 \\
2.6473 E-01 \\
1.1482 E+00 \\
2.7489 E+00 \\
4.1640 E+00 \\
5.5018 E+00 \\
9.3801 E+00 \\
3.49 U 2 E+00 \\
5.0810 E+00 \\
5.6350 E+00 \\
5.4746 E+00 \\
5.7602 E+00 \\
5.6436 E+00 \\
5.1358 E+00 \\
5.0959 E+00 \\
4.2517 E+00 \\
4.3037 E+00 \\
3.4769 E+00 \\
2.9931 E+00 \\
3.8297 E+00 \\
2.9333 E+00 \\
2.6197 E+00 \\
2.0882 E+00 \\
1.4013 E+00 \\
1.3288 E+00 \\
1.0394 E+00 \\
6.0038 E-01 \\
1.3783 E-01 \\
4.5227 E-01 \\
8.7894 E-01 \\
8.9586 E-01 \\
6.3919 E-01 \\
5.0563 E-01 \\
3.8163 E-01 \\
2.4813 E-01 \\
1.6829 E-01 \\
9.6740 E-02 \\
1.2584 E-01 \\
4.3003 E-02 \\
1.0516 E-02 \\
2.3239 E-03 \\
9.6620 E-04 \\
1.3771 E-04 \\
3.0529 E-05 \\
3.5514 E-06 \\
1.8360 E-0 E \\
8.8049 E-07 \\
1.3988 E-07 \\
6.6783 E-09 \\
2.3201 E-10 \\
1.0000 E+02\end{array}$ & $\begin{array}{l}2.2196 E-02 \\
2.6062 E-01 \\
1.1295 E+00 \\
2.7089 E+00 \\
4.1067 E+00 \\
5.4317 E+00 \\
9.3083 E+00 \\
3.4623 E+00 \\
5.0473 E+00 \\
5.6057 E+00 \\
5.4526 E+00 \\
5.7443 E+00 \\
5.6403 E+00 \\
5.1350 E+00 \\
5.1086 E+00 \\
4.2660 E+00 \\
4.3264 E+00 \\
3.4903 E+00 \\
3.0201 E+00 \\
3.8719 E+00 \\
2.9701 E+00 \\
2.6556 E+00 \\
2.1190 E+00 \\
1.4227 E+00 \\
1.3498 E+00 \\
1.0562 E+00 \\
6.1025 E-01 \\
1.4009 E-01 \\
4.5978 E-01 \\
8.9434 E-01 \\
9.1241 E-01 \\
6.5172 E-01 \\
5.1615 E-01 \\
3.9001 E-01 \\
2.5384 E-01 \\
1.7224 E-01 \\
9.9121 E-02 \\
1.2916 E-01 \\
4.4210 E-02 \\
1.0825 E-02 \\
2.3948 E-03 \\
9.9756 E-04 \\
1.4233 E-04 \\
3.1585 E-05 \\
3.6781 E-06 \\
1.9026 E-06 \\
9.1281 E-07 \\
1.4508 E-07 \\
6.9294 E-09 \\
2.4074 E-10 \\
1.0000 E+02\end{array}$ & $\begin{array}{l}2.2570 \mathrm{E}-02 \\
2.6542 \mathrm{E}-01 \\
1.1512 \mathrm{E}+00 \\
2.7555 \mathrm{E}+00 \\
4.1733 \mathrm{E}+00 \\
5.5126 \mathrm{E}+00 \\
9.3885 \mathrm{E}+00 \\
3.4932 \mathrm{E}+00 \\
5.0835 \mathrm{E}+00 \\
5.6352 \mathrm{E}+00 \\
5.4725 \mathrm{E}+00 \\
5.7552 \mathrm{E}+00 \\
5.6360 \mathrm{E}+00 \\
5.1271 \mathrm{E}+00 \\
5.0857 \mathrm{E}+00 \\
4.2426 \mathrm{E}+00 \\
4.2942 \mathrm{E}+00 \\
3.4693 \mathrm{E}+00 \\
2.9868 \mathrm{E}+00 \\
3.8237 \mathrm{E}+00 \\
2.9302 \mathrm{E}+00 \\
2.6187 \mathrm{E}+00 \\
2.0890 \mathrm{E}+00 \\
1.4024 \mathrm{E}+00 \\
1.3305 \mathrm{E}+00 \\
1.0412 \mathrm{E}+00 \\
6.0157 \mathrm{E}-01 \\
1.3810 \mathrm{E}-01 \\
4.5324 \mathrm{E}-01 \\
8.8146 \mathrm{E}-01 \\
8.9942 \mathrm{E}-01 \\
6.4239 \mathrm{E}-01 \\
5.0887 \mathrm{E}-01 \\
3.8465 \mathrm{E}-01 \\
2.5046 \mathrm{E}-01 \\
1.7011 \mathrm{E}-01 \\
9.7877 \mathrm{E}-02 \\
1.2767 \mathrm{E}-01 \\
4.3740 \mathrm{E}-02 \\
1.0721 \mathrm{E}-02 \\
2.3742 \mathrm{E}-03 \\
9.9322 \mathrm{E}-04 \\
1.4191 \mathrm{E}-04 \\
3.1614 \mathrm{E}-05 \\
3.6881 \mathrm{E}-06 \\
1.9700 \mathrm{E}-06 \\
1.0096 \mathrm{E}-06 \\
1.6465 \mathrm{E}-07 \\
7.9141 \mathrm{E}-09 \\
2.7539 \mathrm{E}-10 \\
1.0000 \mathrm{E}+02\end{array}$ \\
\hline
\end{tabular}


TABLE $B-x \times \times 11$

ZPPR-2 EDGE FLUX SPECTRA

\begin{tabular}{|c|c|c|c|c|}
\hline GROUP & $\begin{array}{r}\text { ENERGY } \\
\text { BOUNDRRY } \\
\text { (EV) }\end{array}$ & $\begin{array}{r}\text { DIFFUSION } \\
\text { THEORY } \\
\text { [N/CM2/S }\end{array}$ & $\begin{array}{r}\text { TRANSPORT } \\
\text { THEORY } \\
\text { (N/CM2/S) }\end{array}$ & $\begin{array}{r}\text { OIFFUSION } \\
\text { THEORY } \\
\text { (N/CM } 2 / S \text { I }\end{array}$ \\
\hline $\begin{array}{l}1 \\
2 \\
3 \\
4 \\
5 \\
6 \\
7 \\
8 \\
9 \\
10 \\
11 \\
12 \\
13 \\
14 \\
15 \\
16 \\
17 \\
18 \\
19 \\
20 \\
21 \\
22 \\
23 \\
24 \\
25 \\
26 \\
27 \\
28 \\
29 \\
30 \\
31 \\
32 \\
33 \\
34 \\
35 \\
36 \\
37 \\
38 \\
39 \\
40 \\
41 \\
42 \\
43 \\
44 \\
45 \\
46 \\
47 \\
48 \\
49 \\
50\end{array}$ & $\begin{array}{l}1.9971 E+07 \\
1.0000 E+07 \\
6.0653 E+06 \\
3.6788 E+06 \\
2.2313 E+06 \\
1.3534 E+06 \\
8.2085 E+05 \\
4.9787 E+05 \\
3.8774 E+05 \\
3.0197 E+05 \\
2.3518 E+05 \\
1.8316 E+05 \\
1.4264 E+05 \\
1.1109 E+05 \\
8.6517 E+04 \\
6.7379 E+04 \\
5.2475 E+04 \\
4.0868 E+04 \\
3.1828 E+04 \\
2.4788 E+04 \\
1.9305 E+04 \\
1.5034 E+04 \\
1.1709 E+04 \\
9.1188 E+03 \\
7.1017 E+03 \\
5.5308 E+03 \\
4.3074 E+03 \\
3.3546 E+03 \\
2.6126 E+03 \\
2.0347 E+03 \\
1.5846 E+03 \\
1.2341 E+03 \\
9.6112 E+02 \\
7.4852 E+02 \\
5.8295 E+02 \\
4.5400 E+02 \\
3.5358 E+02 \\
2.7536 E+02 \\
1.6702 E+02 \\
1.0130 E+02 \\
6.1442 E+01 \\
3.7267 E+01 \\
2.2603 E+01 \\
1.3710 E+01 \\
8.3153 E+00 \\
5.0435 E+00 \\
3.0590 E+00 \\
1.8554 E+00 \\
1.1254 E+00 \\
6.8256 E-01 \\
1.0000 E-05\end{array}$ & $\begin{array}{l}1.4373 E-03 \\
1.2345 E-02 \\
4.4946 E-02 \\
2.2639 E-01 \\
5.7969 E-01 \\
1.0742 E+00 \\
6.5018 E+00 \\
2.0094 E+00 \\
3.7433 E+00 \\
3.5140 E+00 \\
3.4258 E+00 \\
4.7808 E+00 \\
5.4893 E+00 \\
2.7548 E+00 \\
5.8721 E+00 \\
3.2153 E+00 \\
2.7592 E+00 \\
1.7991 E+00 \\
7.4259 E+00 \\
1.1613 E+01 \\
4.7255 E+00 \\
3.4155 E+00 \\
2.1517 E+00 \\
6.9091 E-01 \\
1.3147 E+00 \\
2.0367 E+00 \\
1.9142 E+00 \\
1.4567 E+00 \\
9.0612 E-01 \\
1.0539 E+00 \\
1.1770 E+00 \\
8.4430 E-01 \\
9.3047 E-01 \\
8.5743 E-01 \\
8.6454 E-01 \\
7.1743 E-01 \\
4.7613 E-01 \\
1.4355 E+00 \\
1.3671 E+00 \\
1.1885 E+00 \\
1.0026 E+00 \\
8.3899 E-01 \\
6.0749 E-01 \\
4.6204 E-01 \\
3.0660 E-01 \\
1.9428 E-01 \\
1.1474 E-01 \\
6.6138 E-02 \\
2.8586 E-02 \\
1.1334 E-02 \\
1.0000 E+02\end{array}$ & $\begin{array}{l}2.9546 E-03 \\
2.6262 E-02 \\
8.5572 E-02 \\
3.4092 E-01 \\
7.3545 E-01 \\
1.1311 E+00 \\
6.5232 E+00 \\
1.9824 E+00 \\
3.5348 E+00 \\
3.3658 E+00 \\
2.9097 E+00 \\
4.3229 E+00 \\
5.0150 E+00 \\
2.9020 E+00 \\
5.1454 E+00 \\
3.3079 E+00 \\
3.2063 E+00 \\
1.8284 E+00 \\
5.0369 E+00 \\
1.2301 E+01 \\
5.2054 E+00 \\
3.7507 E+00 \\
2.3600 E+00 \\
7.3989 E-01 \\
1.4175 E+00 \\
2.2133 E+00 \\
2.0747 E+00 \\
1.5901 E+00 \\
9.9866 E-01 \\
1.1587 E+00 \\
1.2906 E+00 \\
9.1895 E-01 \\
1.0163 E+00 \\
9.3793 E-01 \\
9.4695 E-01 \\
7.8557 E-01 \\
5.1437 E-01 \\
1.5770 E+00 \\
1.5039 E+00 \\
1.3081 E+00 \\
1.1031 E+00 \\
3.2086 E-01 \\
6.6770 E-01 \\
5.06222 E-01 \\
3.3589 E-01 \\
2.1271 E-01 \\
1.2534 E-01 \\
7.2059 E-02 \\
3.1086 E-02 \\
1.2293 E-02 \\
1.0000 E+02\end{array}$ & $\begin{array}{l}1.4413 E-03 \\
1.2384 E-02 \\
4.5088 E-02 \\
2.2692 E-01 \\
5.8082 E-01 \\
1.0759 E+00 \\
6.5013 E+00 \\
2.0090 E+00 \\
3.742 .3 E+00 \\
3.5120 E+00 \\
3.4225 E+00 \\
4.7746 E+00 \\
5.4816 E+00 \\
2.7503 E+00 \\
5.8611 E+00 \\
3.2088 E+00 \\
2.7531 E+00 \\
1.7950 E+00 \\
7.4120 E+00 \\
1.1615 E+01 \\
4.7262 E+00 \\
3.4156 E+00 \\
2.1516 E+00 \\
6.9085 E-01 \\
1.3145 E+00 \\
2.0366 E+00 \\
1.9145 E+00 \\
1.4571 E+00 \\
9.0643 E-01 \\
1.0544 E+00 \\
1.1778 E+00 \\
8.4503 E-01 \\
9.3154 E-01 \\
8.5870 E-01 \\
8.6614 E-01 \\
7.1898 E-01 \\
4.7726 E-01 \\
1.4404 E+00 \\
1.3733 E+00 \\
1.1953 E+00 \\
1.0096 E+00 \\
8.4593 E-01 \\
6.1322 E-01 \\
4.6695 E-01 \\
3.1018 E-01 \\
1.9674 E-01 \\
1.1629 E-01 \\
6.7091 E-02 \\
2.9021 E-02 \\
1.1512 E-02 \\
1.0000 E+02\end{array}$ \\
\hline
\end{tabular}


TABLE $B-x \times x \times 111$

ZPPR-2 CENTRFLL ADJOINT SPECTRA

\begin{tabular}{|c|c|c|c|c|}
\hline GROUP & $\begin{array}{r}\text { ENERGY } \\
\text { BOUNDRRY } \\
\text { IEVI }\end{array}$ & $\begin{array}{r}1-0 \\
\text { DIFFUSION } \\
\text { THEORY } \\
\text { (RHO/N/S) }\end{array}$ & $\begin{array}{r}1-0 \\
\text { TRANSPORT } \\
\text { THEORY } \\
\text { (RHO } / N / S)\end{array}$ & $\begin{array}{r}2-0 \\
\text { DIFFUSION } \\
\text { THEORY } \\
{[R H O / N / S\}}\end{array}$ \\
\hline $\begin{array}{r}1 \\
2 \\
3 \\
4 \\
5 \\
6 \\
7 \\
8 \\
9 \\
10 \\
11 \\
12 \\
13 \\
14 \\
15 \\
16 \\
17 \\
18 \\
19 \\
20 \\
21 \\
22 \\
23 \\
24 \\
25 \\
26 \\
27 \\
28 \\
29 \\
30 \\
31 \\
32 \\
33 \\
34 \\
35 \\
36 \\
37 \\
38 \\
39 \\
40 \\
41 \\
42 \\
43 \\
44 \\
45 \\
46 \\
47 \\
48 \\
49 \\
50\end{array}$ & $\begin{array}{l}1.9971 E+07 \\
1.0000 E+07 \\
6.0653 E+06 \\
3.6788 E+06 \\
2.2313 E+06 \\
1.3534 E+06 \\
8.2085 E+05 \\
4.9787 E+05 \\
3.8774 E+05 \\
3.0197 E+05 \\
2.3518 E+05 \\
1.8316 E+05 \\
1.4264 E+05 \\
1.1109 E+05 \\
8.6517 E+04 \\
6.7379 E+04 \\
5.2475 E+04 \\
4.0868 E+04 \\
3.1828 E+04 \\
2.4788 E+04 \\
1.9335 E+04 \\
1.5034 E+04 \\
1.1709 E+04 \\
9.1188 E+03 \\
7.1017 E+03 \\
5.5308 E+03 \\
4.3074 E+03 \\
3.3546 E+03 \\
2.6126 E+03 \\
2.0347 E+03 \\
1.5846 E+03 \\
1.2341 E+03 \\
9.6112 E+02 \\
7.4852 E+02 \\
5.8295 E+02 \\
4.5400 E+02 \\
3.5358 E+02 \\
2.7536 E+02 \\
1.6702 E+02 \\
1.0130 E+02 \\
6.1442 E+01 \\
3.7267 E+01 \\
2.2603 E+01 \\
1.3710 E+01 \\
8.3153 E+00 \\
5.0435 E+00 \\
3.0590 E+00 \\
1.8554 E+00 \\
1.1254 E+00 \\
6.8256 E-01 \\
1.0000 E-05\end{array}$ & $\begin{array}{l}1.4185 \mathrm{E}+00 \\
1.2312 \mathrm{E}+00 \\
1.0941 \mathrm{E}+00 \\
1.0989 \mathrm{E}+00 \\
1.0194 \mathrm{E}+00 \\
9.2072 \mathrm{E}-01 \\
9.0042 \mathrm{E}-01 \\
8.7624 \mathrm{E}-01 \\
8.6045 \mathrm{E}-01 \\
8.4430 \mathrm{E}-01 \\
8.3035 \mathrm{E}-01 \\
8.1543 \mathrm{E}-01 \\
7.9952 \mathrm{E}-01 \\
7.8366 \mathrm{E}-01 \\
7.6616 \mathrm{E}-01 \\
7.5020 \mathrm{E}-01 \\
7.3870 \mathrm{E}-01 \\
7.3042 \mathrm{E}-01 \\
7.2618 \mathrm{E}-01 \\
7.2428 \mathrm{E}-01 \\
7.3054 \mathrm{E}-01 \\
7.3617 \mathrm{E}-01 \\
7.4716 \mathrm{E}-01 \\
7.6042 \mathrm{E}-01 \\
7.7040 \mathrm{E}-01 \\
7.8923 \mathrm{E}-01 \\
8.0654 \mathrm{E}-01 \\
8.1611 \mathrm{E}-01 \\
8.1993 \mathrm{E}-01 \\
8.3827 \mathrm{E}-011 \\
8.8225 \mathrm{E}-01 \\
8.7803 \mathrm{E}-01 \\
9.4092 \mathrm{E}-01 \\
9.7756 \mathrm{E}-01 \\
1.0769 \mathrm{E}+00 \\
1.0084 \mathrm{E}+00 \\
1.0348 \mathrm{E}+00 \\
1.1044 \mathrm{E}+00 \\
1.0610 \mathrm{E}+00 \\
1.3381 \mathrm{E}+00 \\
1.0427 \mathrm{E}+00 \\
8.8431 \mathrm{E}-01 \\
1.2153 \mathrm{E}+00 \\
1.5275 \mathrm{E}+00 \\
6.3193 \mathrm{E}-01 \\
1.1299 \mathrm{E}+00 \\
9.4583 \mathrm{E}-01 \\
5.5496 \mathrm{E}-01 \\
4.4716 \mathrm{E}-01 \\
1.8410 \mathrm{E}+00 \\
4.6267 \mathrm{E}+01\end{array}$ & $\begin{array}{l}1.4202 E+00 \\
1.2326 E+00 \\
1.0959 E+00 \\
1.1005 E+00 \\
1.0197 E+00 \\
9.2107 E-01 \\
9.0005 E-01 \\
8.7219 E-01 \\
8.5607 E-01 \\
8.3951 E-01 \\
8.2415 E-01 \\
8.0870 E-01 \\
7.9230 E-01 \\
7.7556 E-01 \\
7.5805 E-01 \\
7.4116 E-01 \\
7.2945 E-01 \\
7.2097 E-01 \\
7.1673 E-01 \\
7.1289 E-01 \\
7.1799 E-01 \\
7.2288 E-01 \\
7.3321 E-01 \\
7.4574 E-01 \\
7.5544 E-01 \\
7.7379 E-01 \\
7.9072 E-01 \\
8.0011 E-01 \\
8.0337 E-01 \\
8.2181 E-01 \\
8.6465 E-01 \\
8.6031 E-01 \\
9.2174 E-01 \\
9.5723 E-01 \\
1.0540 E+00 \\
9.8699 E-01 \\
1.0127 E+00 \\
1.0807 E+00 \\
1.0377 E+00 \\
1.3080 E+00 \\
1.0193 E+00 \\
8.6515 E-01 \\
1.1876 E+00 \\
1.4926 E+00 \\
6.1756 E-01 \\
1.1047 E+00 \\
9.2433 E-01 \\
5.4208 E-01 \\
4.3669 E-01 \\
1.7973 E+00 \\
4.5575 E+01\end{array}$ & $\begin{array}{l}1.4210 \mathrm{E}+00 \\
1.2329 \mathrm{E}+00 \\
1.0951 \mathrm{E}+00 \\
1.0998 \mathrm{0}+00 \\
1.0194 \mathrm{E}+00 \\
9.2001 \mathrm{E}-01 \\
8.9937 \mathrm{E}-01 \\
8.7480 \mathrm{E}-01 \\
8.5901 \mathrm{E}-01 \\
8.4348 \mathrm{E}-01 \\
8.2922 \mathrm{E}-01 \\
8.1448 \mathrm{E}-01 \\
7.9880 \mathrm{E}-01 \\
7.8323 \mathrm{E}-01 \\
7.6595 \mathrm{E}-01 \\
7.5025 \mathrm{E}-01 \\
7.3896 \mathrm{E}-01 \\
7.3090 \mathrm{E}-01 \\
7.2681 \mathrm{E}-01 \\
7.2503 \mathrm{E}-01 \\
7.3155 \mathrm{E}-01 \\
7.3730 \mathrm{E}-01 \\
7.4838 \mathrm{E}-01 \\
7.6170 \mathrm{E}-01 \\
7.7169 \mathrm{E}-01 \\
7.9055 \mathrm{E}-01 \\
8.0788 \mathrm{E}-01 \\
5.1747 \mathrm{E}-01 \\
8.2130 \mathrm{E}-01 \\
8.3967 \mathrm{E}-01 \\
8.8372 \mathrm{E}-01 \\
8.7948 \mathrm{E}-01 \\
9.4247 \mathrm{E}-01 \\
9.7913 \mathrm{E}-01 \\
1.0786 \mathrm{E}+00 \\
1.0100 \mathrm{E}+00 \\
1.0363 \mathrm{E}+00 \\
1.1061 \mathrm{E}+00 \\
1.0625 \mathrm{E}+00 \\
1.3399 \mathrm{E}+00 \\
1.0442 \mathrm{E}+00 \\
8.8558 \mathrm{E}-01 \\
1.2169 \mathrm{E}+00 \\
1.5295 \mathrm{E}+00 \\
6.3279 \mathrm{E}-01 \\
1.1316 \mathrm{E}+00 \\
9.4718 \mathrm{E}-01 \\
5.5568 \mathrm{E}-01 \\
4.4772 \mathrm{E}-01 \\
1.8433 \mathrm{E}+00 \\
4.6309 \mathrm{E}+01\end{array}$ \\
\hline
\end{tabular}


TRBLE $B-x \times x$ IV

ZPR-6-7 CENTRAL FLUX SPECTRA

\begin{tabular}{|c|c|c|c|c|}
\hline GROUP & $\begin{array}{r}\text { ENERGY } \\
\text { BOUNDRRY } \\
\text { (EV) }\end{array}$ & $\begin{array}{r}1-D \\
\text { DIFFUSION } \\
\text { THEORY } \\
\text { (N/CM2/S) }\end{array}$ & $\begin{array}{r}1-0 \\
\text { TRANSPORT } \\
\text { THEORY } \\
\text { [N/CM } / S \text { ] }\end{array}$ & $\begin{array}{r}2-0 \\
\text { DIFFUSION } \\
\text { THEORY } \\
\text { (N/CM2/S) }\end{array}$ \\
\hline $\begin{array}{r}1 \\
2 \\
3 \\
4 \\
5 \\
6 \\
7 \\
8 \\
9 \\
10 \\
11 \\
12 \\
13 \\
14 \\
15 \\
16 \\
17 \\
18 \\
19 \\
20 \\
21 \\
22 \\
23 \\
24 \\
25 \\
26 \\
27 \\
28 \\
29 \\
30 \\
31 \\
32 \\
33 \\
34 \\
35 \\
36 \\
37 \\
38 \\
39 \\
40 \\
41 \\
42 \\
43 \\
44 \\
45 \\
46 \\
47 \\
48 \\
49 \\
50\end{array}$ & $\begin{array}{l}1.9971 E+07 \\
1.0000 E+07 \\
6.0653 E+06 \\
3.6788 E+06 \\
2.2313 E+06 \\
1.3534 E+06 \\
8.2085 E+05 \\
4.9787 E+05 \\
3.8774 E+05 \\
3.0197 E+05 \\
2.3518 E+05 \\
1.8316 E+05 \\
1.4264 E+05 \\
1.1109 E+05 \\
8.6517 E+04 \\
6.7379 E+04 \\
5.2475 E+04 \\
4.0868 E+04 \\
3.1828 E+04 \\
2.4788 E+04 \\
1.9305 E+04 \\
1.5034 E+04 \\
1.1709 E+04 \\
9.1188 E+03 \\
7.1017 E+03 \\
5.5308 E+03 \\
4.3074 E+03 \\
3.3546 E+03 \\
2.6126 E+03 \\
2.0347 E+03 \\
1.5846 E+03 \\
1.2341 E+03 \\
9.6112 E+02 \\
7.4852 E+02 \\
5.8295 E+02 \\
4.5400 E+02 \\
3.5358 E+02 \\
2.7536 E+02 \\
1.6702 E+02 \\
1.0130 E+02 \\
6.1442 E+01 \\
3.7267 E+01 \\
2.2603 E+01 \\
1.3710 E+01 \\
8.3153 E+00 \\
5.0435 E+00 \\
3.0590 E+00 \\
1.8554 E+00 \\
1.1254 E+00 \\
6.8256 E-01 \\
1.0000 E-05\end{array}$ & $\begin{array}{l}2.2480 E-02 \\
2.6455 E-01 \\
1.1471 E+00 \\
2.7510 E+00 \\
4.1703 E+00 \\
5.4674 E+00 \\
9.3414 E+00 \\
3.4870 E+00 \\
5.0815 E+00 \\
5.6433 E+00 \\
5.4900 E+00 \\
5.7456 E+00 \\
5.6262 E+00 \\
5.1115 E+00 \\
5.0816 E+00 \\
4.2363 E+00 \\
4.2916 E+00 \\
3.4705 E+00 \\
2.9846 E+00 \\
3.8380 E+00 \\
2.9408 E+00 \\
2.6285 E+00 \\
2.0994 E+00 \\
1.4078 E+00 \\
1.3387 E+00 \\
1.0488 E+00 \\
6.0578 E-01 \\
1.3925 E-01 \\
4.5782 E-01 \\
8.8921 E-01 \\
9.0910 E-01 \\
6.5019 E-01 \\
5.1640 E-01 \\
3.9121 E-01 \\
2.5624 E-01 \\
1.7465 E-01 \\
1.0086 E-01 \\
1.3229 E-01 \\
4.5779 E-02 \\
1.1306 E-02 \\
2.5292 E-03 \\
1 . C 716 E-03 \\
1.5474 E-04 \\
3.4859 E-05 \\
4.1290 E-06 \\
2.1723 E-06 \\
1.1605 E-06 \\
1.8874 E-07 \\
8.9968 E-09 \\
3.1635 E-10 \\
1.0000 E+02\end{array}$ & $\begin{array}{l}2.2378 E-02 \\
2.6330 E-01 \\
1.1416 E+00 \\
2.7389 E+00 \\
4.1523 E+00 \\
5.4466 E+00 \\
9.3172 E+00 \\
3.4784 E+00 \\
5.0701 E+00 \\
5.6316 E+00 \\
5.4808 E+00 \\
5.7372 E+00 \\
5.6214 E+00 \\
5.1073 E+00 \\
5.0838 E+00 \\
4.2391 E+00 \\
4.2962 E+00 \\
3.4722 E+00 \\
2.9972 E+00 \\
3.8547 E+00 \\
2.9553 E+00 \\
2.6421 E+00 \\
2.1108 E+00 \\
1.4158 E+00 \\
1.3466 E+00 \\
1.0550 E+00 \\
6.0954 E-01 \\
1.4012 E-01 \\
4.6030 E-01 \\
8.9514 E-01 \\
9.1539 E-01 \\
6.5499 E-01 \\
5.2048 E-01 \\
3.9451 E-01 \\
2.5849 E-01 \\
1.7620 E-01 \\
1.0179 E-01 \\
1.3368 E-01 \\
4.6290 E-02 \\
1.1442 E-02 \\
2.5606 E-03 \\
1.0859 E-03 \\
1.5691 E-04 \\
3.5361 E-05 \\
4.1914 E-06 \\
2.2045 E-00 \\
1.1774 E-06 \\
1.9155 E-07 \\
9.1338 E-09 \\
3.2119 E-10 \\
1.0000 E+02\end{array}$ & $\begin{array}{l}2.2800 E-02 \\
2.6855 E-01 \\
1.1661 E+00 \\
2.7885 E+00 \\
4.2091 E+00 \\
5.4953 E+00 \\
9.3451 E+00 \\
3.4835 E+00 \\
5.0743 E+00 \\
5.6381 E+00 \\
5.4892 E+00 \\
5.7387 E+00 \\
5.6166 E+00 \\
5.1075 E+00 \\
5.0677 E+00 \\
4.2258 E+00 \\
4.2860 E+00 \\
3.4785 E+00 \\
2.9888 E+00 \\
3.8177 E+00 \\
2.9347 E+00 \\
2.6217 E+00 \\
2.0989 E+00 \\
1.4125 E+00 \\
1.3379 E+00 \\
1.0443 E+00 \\
6.0036 E-01 \\
1.3786 E-01 \\
4.5482 E-01 \\
8.8460 E-01 \\
9.0419 E-01 \\
6.4717 E-01 \\
5.1270 E-01 \\
3.8754 E-01 \\
2.5323 E-01 \\
1.7257 E-01 \\
9.9535 E-02 \\
1.2889 E-01 \\
4.4283 E-02 \\
1.0851 E-02 \\
2.4076 E-03 \\
1.0138 E-03 \\
1.4498 E-04 \\
3.2416 E-05 \\
3.7959 E-06 \\
1.9966 E-06 \\
9.9369 E-07 \\
1.5860 E-07 \\
7.5687 E-09 \\
2.6268 E-10 \\
1.0000 E+02\end{array}$ \\
\hline
\end{tabular}


TABLE $B-x \times \times V$

ZPR-6-7 EDGE FLUX F :CTRA

\begin{tabular}{|c|c|c|c|c|}
\hline toup & $\begin{array}{r}\text { ENERGY } \\
\text { BOUNDARY } \\
\text { IEVI }\end{array}$ & $\begin{array}{r}1-0 \\
\text { DIFFUSION } \\
\text { THEORY } \\
\text { IN/CM2/SI }\end{array}$ & $\begin{array}{r}1-0 \\
\text { TRANSPORT } \\
\text { THEORY } \\
\text { (N/CM2/S) }\end{array}$ & $\begin{array}{r}2-0 \\
\text { DIFFUSION } \\
\text { THEORY } \\
\text { [N/CM2/S] }\end{array}$ \\
\hline $\begin{array}{l}1 \\
2 \\
3 \\
4 \\
5 \\
6 \\
7 \\
8 \\
9 \\
10 \\
11 \\
12 \\
13 \\
14 \\
15 \\
16 \\
17 \\
18 \\
19 \\
20 \\
21 \\
22 \\
23 \\
24 \\
25 \\
26 \\
27 \\
28 \\
29 \\
30 \\
31 \\
32 \\
33 \\
34 \\
35 \\
36 \\
37 \\
38 \\
39 \\
40 \\
41 \\
42 \\
43 \\
44 \\
45 \\
46 \\
47 \\
48 \\
49 \\
50\end{array}$ & $\begin{array}{l}1.9971 E+07 \\
1.0000 E+07 \\
6.0653 E+06 \\
3.6788 E+06 \\
2.2313 E+06 \\
1.3534 E+06 \\
8.2085 E+05 \\
4.9787 E+05 \\
3.8774 E+05 \\
3.0197 E+05 \\
2.3518 E+05 \\
1.8316 E+05 \\
1.4264 E+05 \\
1.1109 E+05 \\
8.6517 E+04 \\
6.7379 E+04 \\
5.2475 E+04 \\
4.0868 E+04 \\
3.1828 E+04 \\
2.4788 E+04 \\
1.9305 E+04 \\
1.5034 E+04 \\
1.1709 E+04 \\
9.1188 E+03 \\
7.10 ! 7 E+03 \\
5.5308 E+03 \\
4.3074 E+03 \\
3.3546 E+03 \\
2.6126 E+03 \\
2.0347 E+03 \\
1.5846 E+03 \\
1.2341 E+03 \\
9.6112 E+02 \\
7.4852 E+02 \\
5.8295 E+02 \\
4.5400 E+02 \\
3.5358 E+02 \\
2.7536 E+02 \\
1.6702 E+02 \\
1.0130 E+02 \\
6.1442 E+01 \\
3.7267 E+01 \\
2.2603 E+01 \\
1.3710 E+01 \\
8.3153 E+00 \\
5.0435 E+00 \\
3.0590 E+00 \\
1.8554 E+00 \\
1.1254 E+00 \\
6.8256 E-01 \\
1.0000 E-05\end{array}$ & $\begin{array}{l}5.0846 E-03 \\
5.0144 E-02 \\
1.7626 E-01 \\
3.4306 E-01 \\
5.3679 E-01 \\
1.6301 E+00 \\
8.4101 E+00 \\
5.6503 E+00 \\
6.9764 E+00 \\
7.3720 E+00 \\
7.0418 E+00 \\
6.7228 E+00 \\
6.7546 E+00 \\
6.6145 E+00 \\
7.4297 E+00 \\
6.7035 E+00 \\
6.8935 E+00 \\
5.3486 E+00 \\
3.3002 E+00 \\
4.5933 E+00 \\
2.4786 E+00 \\
1.7067 E+00 \\
1.0972 E+00 \\
5.0749 E-01 \\
3.9651 E-01 \\
2.6520 E-01 \\
2.2530 E-01 \\
1.0053 E-01 \\
5.1419 E-02 \\
1.8543 E-01 \\
1.2629 E-01 \\
6.1587 E-02 \\
7.7491 E-02 \\
4.5483 E-02 \\
3.6479 E-02 \\
2.0242 E-02 \\
1.0876 E-02 \\
4.6162 E-02 \\
6.2423 E-03 \\
1.4988 E-03 \\
3.5870 E-04 \\
4.7057 E-05 \\
2.5700 E-06 \\
1.4853 E-06 \\
1.7858 E-08 \\
1.8829 E-08 \\
5.3365 E-08 \\
3.3060 E-08 \\
8.1164 E-09 \\
7.0603 E-10 \\
1.0000 E+02\end{array}$ & $\begin{array}{l}7.1287 E-03 \\
6.7128 E-02 \\
2.2971 E-01 \\
4.4104 E-01 \\
6.8546 E-01 \\
1.9487 E+00 \\
8.8226 E+00 \\
5.8040 E+00 \\
7.0735 E+00 \\
7.4412 E+00 \\
7.0995 E+00 \\
6.7738 E+00 \\
6.7941 E+00 \\
6.6716 E+00 \\
7.4575 E+00 \\
6.7532 E+00 \\
6.7832 E+00 \\
5.1619 E+00 \\
3.12 E 0 E+00 \\
4.2776 E+00 \\
2.3059 E+00 \\
1.5808 E+00 \\
1.0092 E+00 \\
4.7110 E-01 \\
3.6470 E-01 \\
2.4262 E-01 \\
1.9890 E-01 \\
3.9031 E-02 \\
4.1230 E-02 \\
5.6762 E-02 \\
5.6231 E-02 \\
2.9394 E-02 \\
3.9742 E-02 \\
2.6126 E-02 \\
2.5191 E-02 \\
1.6045 E-02 \\
8.5480 E-03 \\
1.5471 E-02 \\
3.0428 E-03 \\
7.0031 E-04 \\
2.2341 E-04 \\
3.0513 E-05 \\
1.6961 E-06 \\
1.0199 E-06 \\
1.5792 E-08 \\
2.2975 E-08 \\
6.2658 E-08 \\
3.7140 E-08 \\
8.9047 E-09 \\
7.7262 E-10 \\
1.0000 E+02\end{array}$ & $\begin{array}{l}4.4871 E-03 \\
4.4794 E-02 \\
1.5923 E-01 \\
3.1322 E-01 \\
4.9356 E-01 \\
1.4962 E+00 \\
7.7343 E+00 \\
5.3334 E+00 \\
6.6372 E+00 \\
7.1301 E+00 \\
6.9636 E+00 \\
6.7351 E+00 \\
6.8355 E+00 \\
6.8406 E+00 \\
7.7197 E+00 \\
7.0128 E+00 \\
7.2057 E+00 \\
5.5883 E+00 \\
3.5535 E+00 \\
4.5925 E+00 \\
2.5261 E+00 \\
1.7318 E+00 \\
1.1147 E+00 \\
5.3647 E-01 \\
4.1131 E-01 \\
2.7306 E-01 \\
2.3286 E-01 \\
1.0245 E-01 \\
5.0070 E-02 \\
1.7693 E-01 \\
1.3204 E-01 \\
6.5516 E-02 \\
7.9449 E-02 \\
4.7907 E-02 \\
3.8612 E-02 \\
2.2421 E-02 \\
1.2222 E-02 \\
4.3931 E-02 \\
6.5669 E-03 \\
1.4187 E-03 \\
3.8009 E-04 \\
4.6168 E-05 \\
2.4502 E-06 \\
1.2077 E-06 \\
1.9651 E-08 \\
2.3685 E-08 \\
6.7059 E-08 \\
3.9638 E-08 \\
9.3657 E-09 \\
8.1180 E-10 \\
1.0000 E+02\end{array}$ \\
\hline
\end{tabular}


TABLE $B-x \times X \times V]$

ZPR-6-7 CENTRAL AOJOINT SPECTRA

\begin{tabular}{|c|c|c|c|c|}
\hline GROUIP & $\begin{array}{r}\text { ENERGY } \\
\text { BOUNDARY } \\
\text { (EV) }\end{array}$ & $\begin{array}{r}1-D \\
\text { OIFFUSION } \\
\text { THEORY } \\
\text { (RHO/N/S) }\end{array}$ & $\begin{array}{r}1-0 \\
\text { TRANSPORT } \\
\text { THEORY } \\
\text { IRHO/N/SI }\end{array}$ & $\begin{array}{r}2-D \\
\text { OIFFUSION } \\
\text { THEORY } \\
\text { \{RHO/N/S }\end{array}$ \\
\hline $\begin{array}{l}1 \\
2 \\
3 \\
4 \\
5 \\
6 \\
7 \\
8 \\
9 \\
10 \\
11 \\
12 \\
13 \\
14 \\
15 \\
16 \\
17 \\
18 \\
19 \\
20 \\
21 \\
22 \\
23 \\
24 \\
25 \\
26 \\
27 \\
28 \\
29 \\
30 \\
31 \\
32 \\
33 \\
34 \\
35 \\
36 \\
37 \\
38 \\
39 \\
40 \\
41 \\
42 \\
43 \\
44 \\
45 \\
46 \\
47 \\
48 \\
49 \\
50\end{array}$ & $\begin{array}{l}1.9971 E+07 \\
1.0000 E+07 \\
6.0653 E+06 \\
3.6789 E+06 \\
2.2313 E+06 \\
1.3534 E+06 \\
8.2085 E+05 \\
4.9787 E+05 \\
3.8774 E+05 \\
3.0197 E+05 \\
2.3518 E+05 \\
1.8316 E+05 \\
1.4264 E+05 \\
1.1109 E+05 \\
8.6517 E+04 \\
6.7379 E+04 \\
5.2475 E+04 \\
4.0868 E+04 \\
3.1828 E+04 \\
2.4788 E+04 \\
1.9305 E+04 \\
1.5034 E+04 \\
1.1709 E+04 \\
9.1188 E+03 \\
7.1017 E+03 \\
5.5308 E+03 \\
4.3074 E+03 \\
3.3546 E+03 \\
2.6126 E+03 \\
2.0347 E+03 \\
1.5846 E+03 \\
1.2341 E+03 \\
9.6112 E+02 \\
7.4852 E+02 \\
5.8295 E+02 \\
4.5400 E+02 \\
3.5358 E+02 \\
2.7536 E+02 \\
1.6702 E+02 \\
1.0130 E+02 \\
6.1442 E+01 \\
3.7267 E+01 \\
2.2603 E+01 \\
1.3710 E+01 \\
8.3153 E+00 . \\
5.0435 E+00 \\
3.0590 E+00 \\
1.8554 E+00 \\
1.1254 E+00 \\
6.8256 E E-01 \\
1.0000 E-05\end{array}$ & $\begin{array}{l}1.4152 E+00 \\
1.2297 E-30 \\
1.0932 E+00 \\
1.0986 E+00 \\
1.0192 E+00 \\
9.2098 E-01 \\
9.0087 E-01 \\
8.7712 E-01 \\
8.6146 E-01 \\
8.4604 E-01 \\
8.3186 E-01 \\
8.1714 E-01 \\
8.0144 E-01 \\
7.8581 E-01 \\
7.6851 E-01 \\
7.5275 E-01 \\
7.4140 E-01 \\
7.3319 E-01 \\
7.2898 E-01 \\
7.2715 E-01 \\
7.3350 E-01 \\
7.3918 E-01 \\
7.5024 E-01 \\
7.6354 E-01 \\
7.7353 E-01 \\
7.9234 E-01 \\
8.0960 E-01 \\
8.1916 E-01 \\
8.2297 E-01 \\
8.4131 E-01 \\
8.8530 E-01 \\
8.8130 E-01 \\
9.4499 E-01 \\
9.8200 E-01 \\
1.0814 E+00 \\
1.0112 E+00 \\
1.0374 E+00 \\
1.1073 E+00 \\
1.0649 E+00 \\
1.3418 E+00 \\
1.0455 E+00 \\
8.8389 E-01 \\
1.2189 E+00 \\
1.5296 E+00 \\
6.3338 E-01 \\
1.1747 E+00 \\
1.0361 E+00 \\
5.6260 E-01 \\
4.4492 E-01 \\
1.8471 E+00 \\
4.6510 E+01\end{array}$ & $\begin{array}{l}1.4143 E+00 \\
1.2289 E+00 \\
1.0928 E+00 \\
1.0983 E+00 \\
1.0191 E+00 \\
9.2155 E-01 \\
9.0138 E-01 \\
8.7733 E-01 \\
8.6146 E-01 \\
8.4598 E-01 \\
8.3186 E-01 \\
8.1709 E-01 \\
8.0146 E-01 \\
7.8572 E-01 \\
7.6870 E-01 \\
7.5241 E-01 \\
7.4110 E-01 \\
7.3305 E-01 \\
7.2908 E-01 \\
7.2511 E-01 \\
7.3142 E-01 \\
7.3687 E-01 \\
7.4791 E-0 ! \\
7.6 ! 20 E-01 \\
7.7112 E-01 \\
7.8990 E-01 \\
8.0717 E-01 \\
8.1670 E-01 \\
8.2048 E-01 \\
8.3861 E-01 \\
8.8226 E-01 \\
8.7830 E-01 \\
9.4161 E-01 \\
9.7837 E-01 \\
1.0772 E+00 \\
1.0075 E+00 \\
1.0338 E+00 \\
1.1031 E+00 \\
1.0607 E+00 \\
1.3360 E+00 \\
1.0410 E+00 \\
8.8044 E-01 \\
1.2135 E+00 \\
1.5225 E+00 \\
6.3049 E-01 \\
1.1687 E+00 \\
1.0308 E+00- \\
5.5988 E-01 \\
4.4273 E-01 \\
1.8373 E+00 \\
4.6394 E+01\end{array}$ & $\begin{array}{l}1.4197 E+00 \\
1.2326 E+00 \\
1.0944 E+00 \\
1.0992 E+00 \\
1.0191 E+00 \\
9.2014 E-01 \\
8.9958 E-01 \\
8.7634 E-01 \\
8.6076 E-01 \\
8.4551 E-01 \\
8.3152 E-01 \\
8.1696 E-01 \\
8.0146 E-01 \\
7.8598 E-01 \\
7.6876 E-01 \\
7.5309 E-01 \\
7.4176 E-01 \\
7.3361 E-01 \\
7.2937 E-01 \\
7.2749 E-01 \\
7.3386 E-01 \\
7.3959 E-01 \\
7.5054 E-01 \\
7.6404 E-01 \\
7.7405 E-01 \\
7.9298 E-01 \\
8.1039 E-01 \\
8.1997 E-01 \\
8.2377 E-01 \\
8.4221 E-01 \\
8.8625 E-01 \\
8.8241 E-01 \\
9.4437 E-01 \\
9.8164 E-01 \\
1.0823 E+00 \\
1.0115 E+00 \\
1.0379 E+00 \\
1.1072 E+00 \\
1.0643 E+00 \\
1.3433 E+00 \\
1.0451 E+00 \\
8.7995 E-01 \\
1.2180 E+00 \\
1.5299 E+00 \\
6.2469 E-01 \\
1.1314 E+00 \\
9.6253 E-01 \\
5.5763 E-01 \\
4.4914 E-01 \\
1.8498 E+00 \\
4.6398 E+01\end{array}$ \\
\hline
\end{tabular}


TABLE B-XXXVII

ZPR-3-56B CENTRAL FLUX SPECTRA

\begin{tabular}{|c|c|c|c|c|}
\hline GROUP & $\begin{array}{r}\text { ENERGY } \\
\text { BOUNORRY } \\
\text { (EV| }\end{array}$ & $\begin{array}{r}1-D \\
\text { OIFFUSION } \\
\text { THEORY } \\
\text { IN/CM } 2 / S I\end{array}$ & $\begin{array}{r}1-D \\
\text { TRANSPORT } \\
\text { THEORY } \\
\text { (N/CM2/S) }\end{array}$ & $\begin{array}{r}2-0 \\
\text { DIFFUSION } \\
\text { THEORY } \\
\text { IN/CM2/SI }\end{array}$ \\
\hline $\begin{array}{r}1 \\
2 \\
3 \\
4 \\
5 \\
6 \\
7 \\
8 \\
9 \\
10 \\
11 \\
12 \\
13 \\
14 \\
15 \\
16 \\
17 \\
18 \\
19 \\
20 \\
21 \\
22 \\
23 \\
24 \\
25 \\
26 \\
27 \\
28 \\
29 \\
30 \\
31 \\
32 \\
33 \\
34 \\
35 \\
36 \\
37 \\
38 \\
39 \\
40 \\
41 \\
42 \\
43 \\
44 \\
45 \\
46 \\
47 \\
48 \\
49 \\
50\end{array}$ & $\begin{array}{l}1.9371 E+07 \\
1.0000 E+07 \\
6.0653 E+06 \\
3.678 B E+06 \\
2.2313 E+06 \\
1.3534 E+06 \\
8.2085 E+05 \\
4.9787 E+05 \\
3.8774 E+05 \\
3.0197 E+05 \\
2.3518 E+05 \\
1.8316 E+05 \\
1.4264 E+05 \\
1.1103 E+05 \\
0.6517 E+04 \\
6.7379 E+04 \\
5.2475 E+04 \\
4.0868 E+04 \\
3.12 \\
2.4788 E+04 \\
1.9305 E+04 \\
1.5034 E+04 \\
1.1709 E+04 \\
9.1188 E+03 \\
7.1017 E+03 \\
5.5308 E+03 \\
4.3074 E+03 \\
3.3546 E+03 \\
2.6126 E+03 \\
2.0347 E+03 \\
1.5846 E+03 \\
1.2341 E+03 \\
9.6112 E+02 \\
7.4852 E+02 \\
5.8295 E+02 \\
4.5400 E+02 \\
3.5358 E+02 \\
2.7536 E+02 \\
1.6702 E+02 \\
1.0130 E+02 \\
6.1442 E+01 \\
3.7267 E+01 \\
2.2603 E+01 \\
1.3710 E+01 \\
8.3153 E+00 \\
5.0435 E+00 \\
3.0590 E+00 \\
1.8554 E+00 \\
1.1254 E+00 \\
6.8256 E-01 \\
1.0000 E-05\end{array}$ & $\begin{array}{l}2.7001 E-02 \\
3.1913 E-01 \\
1.3793 E+00 \\
3.2048 E+00 \\
4.7430 E+00 \\
6.4434 E+00 \\
1.0353 E+01 \\
3.9337 E+00 \\
5.5927 E+00 \\
6.0983 E+00 \\
5.8771 E+00 \\
5.9923 E-00 \\
5.7498 E+00 \\
5.1988 E+00 \\
4.9767 E+00 \\
4.1645 E+00 \\
4.1025 E+00 \\
3.2564 E+00 \\
2.7430 E+00 \\
3.3637 E+00 \\
2.5635 E+00 \\
2.1565 E+00 \\
1.6606 E+00 \\
1.1257 E+00 \\
1.0465 E+00 \\
7.9803 E-01 \\
4.5984 E-01 \\
1.1039 E-J 1 \\
3.3735 E-01 \\
5.8553 E-01 \\
5.4860 E-01 \\
3.6796 E-01 \\
2.6671 E-01 \\
1.8540 E-01 \\
1.0798 E-01 \\
6.5207 E-02 \\
3.5227 E-02 \\
3.9358 E-02 \\
1.1415 E-02 \\
2.6517 E-03 \\
4.5130 E-04 \\
2.8275 E-04 \\
3.9339 E-05 \\
9.9850 E-06 \\
1.2568 E-06 \\
2.4322 E-06 \\
4.6947 E-06 \\
8.2249 E-07 \\
3.4851 E-08 \\
9.3934 E-10 \\
1.0000 E+02\end{array}$ & $\begin{array}{l}2.5950 E-02 \\
3.0615 E-01 \\
1.3228 E+00 \\
3.0828 E+00 \\
4.5727 E+00 \\
6.2350 E+00 \\
1.0126 E+01 \\
3.8563 E+00 \\
5.4937 E+00 \\
6.0276 E+00 \\
5.8182 E+00 \\
5.9593 E+00 \\
5.7493 E+00 \\
5.2210 E+00 \\
5.0226 E+00 \\
4.2243 E+00 \\
4.1818 E+00 \\
3.3265 E+00 \\
2.2261 E+00 \\
3.4841 E+00 \\
2.6728 E+00 \\
2.2587 E+00 \\
1.7457 E+00 \\
1.1858 E+00 \\
1.1051 E+00 \\
8.4451 E-01 \\
4.8720 E-01 \\
1.1697 E-01 \\
3.5777 E-01 \\
6.2275 E-01 \\
5.8557 E-01 \\
3.9398 E-01 \\
2.8648 E-01 \\
1.9975 E-01 \\
1.1659 E-01 \\
7.0620 E-02 \\
3.8158 E-02 \\
4.2751 E-02 \\
1.2414 E-02 \\
2.8899 E-03 \\
4.9093 E-04 \\
3.0826 E-04 \\
4.2717 E-05 \\
1.0831 E-05 \\
1.3482 E-06 \\
1.8475 E-06 \\
2.5978 E-06 \\
4.2969 E-07 \\
1.7749 E-08 \\
4.7698 E-10 \\
1.0000 E+02\end{array}$ & $\begin{array}{l}2.7320 E-02 \\
3.2304 E-01 \\
1.3962 E+00 \\
3.2410 E+00 \\
4.7932 E+00 \\
6.5036 E+00 \\
1.0416 E+01 \\
3.9538 E+00 \\
5.6202 E+00 \\
6.1197 E+00 \\
5.8908 E+00 \\
5.9979 E+00 \\
5.7463 E+00 \\
5.1890 E+00 \\
4.9599 E+00 \\
4.1457 E+00 \\
4.0786 E+00 \\
3.2339 E+00 \\
2.7216 E+00 \\
3.3310 E+00 \\
2.5351 E+00 \\
2.1298 E+00 \\
1.6382 E+00 \\
1.1099 E+00 \\
1.0311 E+00 \\
7.8584 E-01 \\
4.5269 E-01 \\
1.0857 E-01 \\
3.3203 E-01 \\
5.7590 E-01 \\
5.3911 E-01 \\
3.6137 E-01 \\
2.5177 E-01 \\
1.8190 E-01 \\
1.0592 E-01 \\
6.3959 E-02 \\
3.4555 E-02 \\
3.8625 E-02 \\
1.1210 E-02 \\
2.6063 E-03 \\
4.4388 E-04 \\
2.8088 E-04 \\
3.9251 E-05 \\
1.0071 E-05 \\
1.2780 E-06 \\
3.8071 E-06 \\
8.2685 E-06 \\
1.4593 E-06 \\
6.1912 E-08 \\
1.6692 E-09 \\
1.0000 E+02\end{array}$ \\
\hline
\end{tabular}


TABLE $B-x \times x \times 1 \| 1$

ZPR-3-56B EDGE FLUX SPECTRA

\begin{tabular}{|c|c|c|c|c|}
\hline GROUP & $\begin{array}{r}\text { ENERGY } \\
\text { BOUNDARY } \\
\text { (EVI }\end{array}$ & $\begin{array}{r}1-0 \\
\text { DIFFUSION } \\
\text { THEORY } \\
\text { IN/CM2/S }\end{array}$ & $\begin{array}{r}1-0 \\
\text { TRANSPORT } \\
\text { THEORY } \\
\text { (N/CM2/S) }\end{array}$ & $\begin{array}{r}2-0 \\
\text { DIFFUSION } \\
\text { THEORY } \\
\text { (N/CM2/SI }\end{array}$ \\
\hline $\begin{array}{l}1 \\
2 \\
3 \\
4 \\
5 \\
5 \\
7 \\
8 \\
9 \\
10 \\
11 \\
12 \\
13 \\
14 \\
15 \\
16 \\
17 \\
18 \\
19 \\
20 \\
21 \\
22 \\
23 \\
24 \\
25 \\
26 \\
27 \\
28 \\
29 \\
30 \\
31 \\
32 \\
33 \\
34 \\
35 \\
36 \\
37 \\
38 \\
39 \\
40 \\
41 \\
42 \\
43 \\
44 \\
45 \\
46 \\
47 \\
48 \\
49 \\
50\end{array}$ & $\begin{array}{l}1.9971 E+07 \\
1.0000 E+07 \\
6.0653 E+06 \\
3.6788 E+06 \\
2.2313 E+06 \\
1.3534 E+06 \\
8.2085 E+05 \\
4.9787 E+05 \\
3.8774 E+05 \\
3.0197 E+05 \\
2.3518 E+05 \\
1.8316 E+05 \\
1.4264 E+05 \\
1.1109 E+05 \\
8.6517 E+04 \\
6.7379 E+04 \\
5.2475 E+04 \\
4.0868 E+04 \\
3.1828 E+04 \\
2.4788 E+04 \\
1.9305 E+04 \\
1.5034 E+04 \\
1.1709 E+04 \\
9.1188 E+03 \\
7.1017 E+03 \\
5.5308 E+03 \\
4.3074 E+03 \\
3.3546 E+03 \\
2.6126 E+03 \\
2.0347 E+03 \\
1.5846 E+03 \\
1.2341 E+03 \\
9.6112 E+02 \\
7.4852 E+02 \\
5.8295 E+02 \\
4.5400 E+02 \\
3.5358 E+02 \\
2.7536 E+02 \\
1.6702 E+02 \\
1.0130 E+02 \\
6.1442 E+01 \\
3.7267 E+01 \\
2.2603 E+01 \\
1.3710 E+01 \\
8.3153 E+00 \\
5.0435 E+00 \\
3.0590 E+00 \\
1.8554 E+00 \\
1.1254 E+00 \\
6.8256 E-01 \\
1.0000 E-05\end{array}$ & $\begin{array}{l}1.3902 E-03 \\
1.0900 E-02 \\
3.0443 E-02 \\
1.4361 E-01 \\
9.5235 E-01 \\
2.6620 E+00 \\
1.0536 E+01 \\
4.9984 E+00 \\
4.9519 E+00 \\
4.3924 E+00 \\
4.2428 E+00 \\
6.3052 E+00 \\
8.8901 E+00 \\
5.7331 E+00 \\
4.0359 E+00 \\
5.7098 E+00 \\
4.7879 E+00 \\
2.8154 E+00 \\
1.5910 E+00 \\
1.4675 E+00 \\
5.0538 E-01 \\
5.1555 E-01 \\
1.6580 E+00 \\
1.6366 E+00 \\
1.1838 E+00 \\
6.0142 E-01 \\
5.9292 E-01 \\
1.8469 E-01 \\
4.8240 E-01 \\
9.1521 E-01 \\
1.0749 E+00 \\
9.5862 E-01 \\
9.9755 E-01 \\
9.3672 E-01 \\
9.3655 E-01 \\
8.3899 E-01 \\
6.3214 E-01 \\
1.6097 E+00 \\
1.5407 E+00 \\
1.4494 E+00 \\
1.3268 E+00 \\
1.2013 E+00 \\
1.0478 E+00 \\
8.8502 E-01 \\
7.0827 E-01 \\
5.3688 E-01 \\
3.7243 E-01 \\
2.4900 E-01 \\
1.1794 E-01 \\
4.5278 E-02 \\
1.0000 E+02\end{array}$ & $\begin{array}{l}3.8792 E-03 \\
3.4824 E-02 \\
1.0502 E-01 \\
3.5736 E-01 \\
1.4866 E+00 \\
3.3808 E+00 \\
1.1061 E+01 \\
5.0054 E+00 \\
4.9590 E+00 \\
4.2966 E+00 \\
4.1654 E+00 \\
5.7153 E+00 \\
7.9652 E+00 \\
5.3599 E+00 \\
3.9213 E+00 \\
4.9514 E+00 \\
4.6085 E+00 \\
2.8037 E+00 \\
1.5606 E+00 \\
1.4728 E+00 \\
4.8065 E-01 \\
4.9426 E-01 \\
1.6498 E+00 \\
1.6773 E+00 \\
1.2150 E+00 \\
6.0468 E-01 \\
6.0204 E-01 \\
1.8413 E-01 \\
4.9370 E-01 \\
9.6056 E-01 \\
1.1351 E+00 \\
1.0129 E+00 \\
1.0598 E+00 \\
9.9815 E-01 \\
1.0011 E+00 \\
8.9645 E-01 \\
6.6840 E-01 \\
1.7288 E+00 \\
1.6552 E+00 \\
1.5514 E+00 \\
1.4121 E+00 \\
1.2669 E+00 \\
1.0942 E+00 \\
9.1283 E-01 \\
7.2119 E-01 \\
5.3925 E-01 \\
3.6899 E-01 \\
2.4325 E-01 \\
1.1400 E-01 \\
4.3296 E-02 \\
1.0000 E+02\end{array}$ & $\begin{array}{l}1.8180 E-03 \\
1.4664 E-02 \\
4.1267 E-02 \\
1.8746 E-01 \\
1.1731 E+00 \\
3.3087 E+00 \\
1.1928 E+01 \\
5.3544 E+00 \\
5.1013 E+00 \\
4.4714 E+00 \\
4.3390 E+00 \\
6.4755 E+00 \\
9.0234 E+00 \\
5.6204 E+00 \\
3.8924 E+00 \\
5.7699 E+00 \\
4.6261 E+00 \\
2.6384 E+00 \\
1.4636 E+00 \\
1.3078 E+00 \\
4.4364 E-01 \\
4.5684 E-01 \\
1.5389 E+00 \\
1.5503 E+00 \\
1.0949 E+00 \\
5.5635 E-01 \\
5.9246 E-01 \\
1.9527 E-01 \\
4.7091 E-01 \\
8.3956 E-01 \\
9.6856 E-01 \\
8.6264 E-01 \\
8.9388 E-01 \\
8.3830 E-01 \\
8.3965 E-01 \\
7.5879 E-01 \\
5.7489 E-01 \\
1.4429 E+00 \\
1.3774 E+00 \\
1.2917 E+00 \\
1.1780 E+00 \\
1.0616 E+00 \\
9.2088 E-01 \\
7.7290 E-01 \\
6.1421 E-01 \\
4.6199 E-01 \\
3.1781 E-01 \\
2.1051 E-01 \\
9.8811 E-02 \\
3.7436 E-02 \\
1.0000 E+02\end{array}$ \\
\hline
\end{tabular}


TABLE $B-x \times \times 1 x$

ZPR-3-56B CENTRAL AOJOINT SPECTRA

\begin{tabular}{|c|c|c|c|c|}
\hline GROUP & $\begin{array}{r}\text { ENERGY } \\
\text { BOUNDRRY } \\
\text { IEV| }\end{array}$ & $\begin{array}{r}\text { I-D } \\
\text { DIFFUSION } \\
\text { THCORY } \\
\text { IRHO/N/SI }\end{array}$ & $\begin{array}{r}1-D \\
\text { TRANSPORT } \\
\text { THEURY } \\
\text { (RHO/PN/SI }\end{array}$ & $\begin{array}{r}2-D \\
\text { OIFFUSION } \\
\text { THEORY } \\
\text { IRHO/N/SI }\end{array}$ \\
\hline $\begin{array}{l}1 \\
2 \\
3 \\
4 \\
5 \\
6 \\
7 \\
8 \\
9 \\
10 \\
11 \\
12 \\
13 \\
14 \\
15 \\
16 \\
17 \\
18 \\
19 \\
20 \\
21 \\
22 \\
23 \\
24 \\
25 \\
26 \\
27 \\
28 \\
29 \\
30 \\
31 \\
32 \\
33 \\
34 \\
35 \\
36 \\
37 \\
38 \\
39 \\
40 \\
41 \\
42 \\
13 \\
44 \\
45 \\
46 \\
47 \\
48 \\
49 \\
50\end{array}$ & $\begin{array}{l}1.9971 E+07 \\
1.0000 E+07 \\
6.0653 E+06 \\
3.6788 E+06 \\
2.2313 E+06 \\
1.3534 E+06 \\
8.2085 E+05 \\
4.9787 E+05 \\
3.8774 E+05 \\
3.0197 E+05 \\
2.3518 E+05 \\
1.8316 E+05 \\
1.4264 E+05 \\
1.1109 E+05 \\
8.6517 E+04 \\
6.7379 E+04 \\
5.2475 E+04 \\
4.0868 E+04 \\
3.1828 E+04 \\
2.4788 E+04 \\
1.9305 E+04 \\
1.5034 E+04 \\
1.1709 E+04 \\
9.1188 E+03 \\
7.1017 E+03 \\
5.5308 E+03 \\
4.3074 E+03 \\
3.3546 E+03 \\
2.6126 E+03 \\
2.0347 E+03 \\
1.5846 E+03 \\
1.2341 E+03 \\
9.6112 E+02 \\
7.4852 E+02 \\
5.8295 E+02 \\
4.5400 E+02 \\
3.5358 E+02 \\
2.7536 E+02 \\
1.6702 E+02 \\
1.0130 E+02 \\
6.1442 E+01 \\
3.7267 E+01 \\
2.2603 E+01 \\
1.3710 E+01 \\
8.3153 E+00 \\
5.0435 E+00 \\
3.0590 E+00 \\
1.8554 E+00 \\
1.1254 E+00 \\
6.8256 E-01 \\
1.0000 E+05\end{array}$ & $\begin{array}{l}1.4379 E+00 \\
1.2183 E+00 \\
1.0839 E+00 \\
1.0873 E+00 \\
1.0221 E+00 \\
9.3663 E-01 \\
9.1662 E-01 \\
9.0175 E-01 \\
8.8498 E-01 \\
8.7079 E-01 \\
8.5824 E-01 \\
8.4337 E-01 \\
8.2728 E-01 \\
8.1044 E-01 \\
7.8999 E-01 \\
7.7135 E-01 \\
7.5601 E-01 \\
7.4363 E-01 \\
7.3460 E-01 \\
7.2800 E-01 \\
7.3293 E-01 \\
7.3712 E-01 \\
7.4682 E-01 \\
7.5987 E-01 \\
7.6761 E-01 \\
7.8755 E-01 \\
8.0727 E-01 \\
8.1744 E-01 \\
8.2057 E-01 \\
8.4179 E-01 \\
8.9054 E-01 \\
8.7454 E-01 \\
9.0784 E-01 \\
9.4388 E-01 \\
1.0606 E+00 \\
9.7873 E-01 \\
1.0667 E+00 \\
1.1394 E+00 \\
1.0402 E+00 \\
1.3778 E+00 \\
9.4956 E-01 \\
9.2814 E-01 \\
13025 E+00 \\
1.5234 E+00 \\
7.3928 E-01 \\
1.2759 E+00 \\
1.1717 E+00 \\
6.1438 E-01 \\
4.6197 E-01 \\
1.9064 E+00 \\
4.7196 E+01\end{array}$ & $\begin{array}{l}1.4341 E+00 \\
1.2163 E+00 \\
1.0850 E+00 \\
1.0884 E+00 \\
1.0227 E+00 \\
9.3909 E-01 \\
9.1827 E-01 \\
8.9738 E-01 \\
8.7973 E-01 \\
8.6365 E-01 \\
8.4918 E-01 \\
8.3303 E-01 \\
8.1581 E-01 \\
7.9755 E-01 \\
7.7682 E-01 \\
7.5648 E-01 \\
7.4059 E-01 \\
7.2766 E-01 \\
7.1849 E-01 \\
7.0881 E-01 \\
7.1105 E-01 \\
7.1327 E-01 \\
7.2094 E-01 \\
7.3187 E-01 \\
7.3884 E-01 \\
7.5724 E-01 \\
7.7560 E-01 \\
7.8526 E-01 \\
7.8840 E-01 \\
8.0868 E-01 \\
8.5444 E-01 \\
8.3812 E-01 \\
8.6967 E-01 \\
9.0311 E-01 \\
1.0129 E+00 \\
9.3480 E-01 \\
1.0181 E+00 \\
1.0870 E+00 \\
9.9115 E-01 \\
1.3108 E+00 \\
9.0367 E-01 \\
8.8562 E E-01 \\
1.2387 E+00 \\
1.4496 E+00 \\
7.0608 E-01 \\
1.2181 E+00 \\
1.1162 E+00 \\
5.8349 E-01 \\
4.3823 E-01 \\
1.8071 E+00 \\
4.5767 E+01\end{array}$ & $\begin{array}{l}1.4392 E+00 \\
1.2190 E+00 \\
1.0836 E+00 \\
1.0870 E+00 \\
1.0219 E+00 \\
9.3588 E-01 \\
9.1602 E-01 \\
9.0302 E-01 \\
8.8649 E-01 \\
8.7298 E-01 \\
8.6123 E-01 \\
8.4688 E-01 \\
8.3132 E-01 \\
8.1503 E-01 \\
7.9484 E-01 \\
7.7655 E-01 \\
7.6140 E-01 \\
7.4927 E-01 \\
7.4040 E-01 \\
7.3387 E-01 \\
7.3962 E-01 \\
7.4438 E-01 \\
7.5474 E-01 \\
7.6850 E-01 \\
7.7649 E-01 \\
7.9694 E-01 \\
8.1715 E-01 \\
8.2749 E-01 \\
8.3060 E-01 \\
8.5205 E-01 \\
9.0166 E-01 \\
8.8580 E-01 \\
9.1959 E-01 \\
9.5645 E-01 \\
1.0754 E+00 \\
9.9234 E-01 \\
1.0819 E+00 \\
1.1556 E+00 \\
1.0554 E+00 \\
1.3984 E+00 \\
9.6371 E-01 \\
9.4127 E-01 \\
1.3223 E+00 \\
1.5462 E+00 \\
7.4996 E-01 \\
1.2930 E+00 \\
1.1883 E+00 \\
6.2381 E-01 \\
4.6921 E-01 \\
1.9364 E+00 \\
4.7640 E+01\end{array}$ \\
\hline
\end{tabular}


TABLE B-XL

SEFOR CENTRAL FLUX SPECTRA

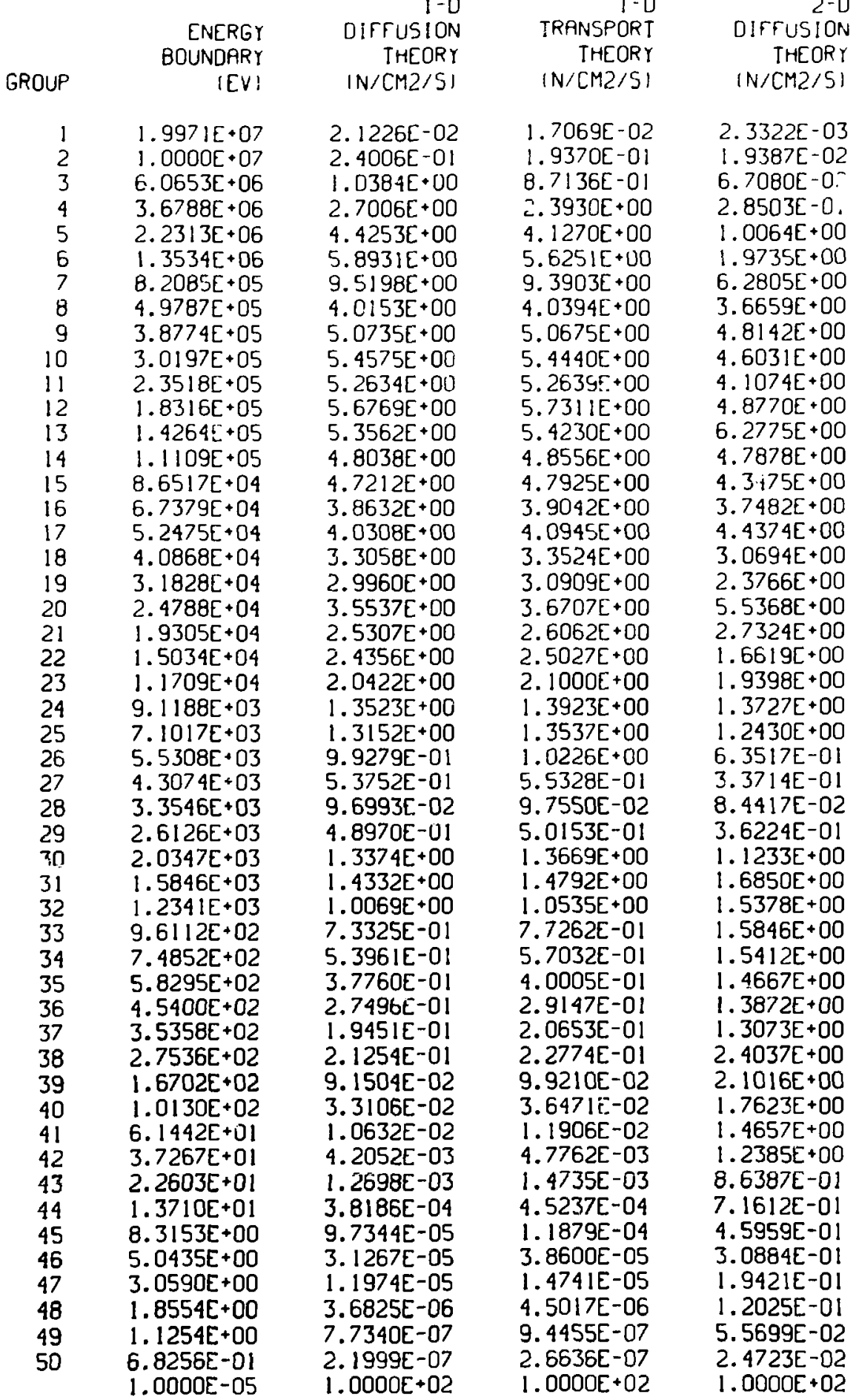


TAELC B-XLI

SETOR COGE TLUX SPECTRA

\begin{tabular}{|c|c|c|c|c|}
\hline GROUP & $\begin{array}{r}\text { EPJERGY } \\
\text { BOUNDRRY } \\
\text { IEVI }\end{array}$ & $\begin{array}{r}1-D \\
\text { OIFFUSION } \\
\text { THEORY } \\
\text { IN/CM2/SI }\end{array}$ & $\begin{array}{r}1-D \\
\text { TRRNSPORT } \\
\text { THEORY } \\
\text { (N/CM2/S) }\end{array}$ & $\begin{array}{r}2-0 \\
\text { DIFFUSION } \\
\text { THEORY } \\
\text { (N/CM2/S) }\end{array}$ \\
\hline $\begin{array}{r}1 \\
2 \\
3 \\
4 \\
5 \\
6 \\
7 \\
8 \\
9 \\
10 \\
11 \\
12 \\
13 \\
14 \\
15 \\
16 \\
17 \\
18 \\
19 \\
20 \\
21 \\
22 \\
23 \\
24 \\
25 \\
26 \\
27 \\
28 \\
29 \\
30 \\
31 \\
32 \\
33 \\
34 \\
35 \\
36 \\
37 \\
38 \\
39 \\
40 \\
41 \\
42 \\
43 \\
44 \\
45 \\
46 \\
47 \\
48 \\
49 \\
50\end{array}$ & $\begin{array}{l}1.9971 E+07 \\
1.0000 E+07 \\
6.0653 E+06 \\
3.6788 E+06 \\
2.2313 E+06 \\
1.3534 E+06 \\
8.2085 E+05 \\
4.9787 E+05 \\
3.8774 E+05 \\
3.0197 E+05 \\
2.3518 E+05 \\
1.8316 E+05 \\
1.4264 E+05 \\
1.1109 E+05 \\
8.6517 E+04 \\
6.7379 E+04 \\
5.2475 E+04 \\
4.0868 E+04 \\
3.1828 E+04 \\
2.4788 E+04 \\
1.9305 E+04 \\
1.5034 E+04 \\
1.1709 E+04 \\
9.1188 E+03 \\
7.1017 E+03 \\
5.5308 E+03 \\
4.3074 E+03 \\
3.3546 E+03 \\
2.6126 E+03 \\
2.0347 E+03 \\
1.5846 E+03 \\
1.2341 E+03 \\
9.6112 E+02 \\
7.4852 E+02 \\
5.8295 E+02 \\
4.5400 E+02 \\
3.5358 E+02 \\
2.7536 E+02 \\
1.6702 E+02 \\
1.0130 E+02 \\
6.1442 E+01 \\
3.7267 E+01 \\
2.2603 E+01 \\
1.3710 E+01 \\
8.3153 E+00 \\
5.0435 E+00 \\
3.0590 E+00 \\
1.8554 E+00 \\
1.1254 E+00 \\
6.8256 E-01 \\
1.0000 E-05\end{array}$ & $\begin{array}{l}5.1424 E-03 \\
4.1273 E-02 \\
1.1999 E-01 \\
4.0904 E-01 \\
1.7880 E+00 \\
3.6240 E+00 \\
1.1049 E+01 \\
4.7488 E+00 \\
6.7453 E+00 \\
6.6374 E+00 \\
5.9155 E+00 \\
5.5128 E+00 \\
6.2583 E+00 \\
5.6931 E+00 \\
5.8911 E+00 \\
6.0115 E+00 \\
5.3646 E+00 \\
4.0261 E+00 \\
4.0375 E+00 \\
3.9211 E+00 \\
3.0842 E+00 \\
2.3652 E+00 \\
1.8356 E+00 \\
1.2972 E+00 \\
1.0270 E+00 \\
7.7816 E-01 \\
5.3096 E-01 \\
2.9537 E-01 \\
2.9666 E-01 \\
2.2744 E-01 \\
1.6172 E-01 \\
1.0988 E-01 \\
7.5615 E-02 \\
5.0313 E-02 \\
3.2192 E-02 \\
1.9706 E-02 \\
1.1521 E-02 \\
9.0188 E-03 \\
2.5960 E-03 \\
5.8602 E-04 \\
1.0450 E-04 \\
1.4596 E-05 \\
1.4927 E-00 \\
1.2762 E-07 \\
7.7893 E-09 \\
3.9202 E-10 \\
1.6109 E-11 \\
5.6746 E-13 \\
1.5912 E-14 \\
7.0341 E-17 \\
1.0000 E+02\end{array}$ & $\begin{array}{l}8.2528 E-03 \\
7.3149 E-02 \\
2.2010 E-01 \\
6.2702 E-01 \\
2.1518 E+00 \\
3.9361 E+00 \\
1.1177 E+01 \\
4.7794 E+00 \\
6.6575 E+00 \\
6.5024 E+00 \\
5.8405 E+00 \\
5.4644 E+00 \\
6.1879 E+00 \\
5.5889 E+00 \\
5.7169 E+00 \\
5.8536 E+00 \\
5.2473 E+00 \\
3.9519 E+00 \\
3.8571 E+00 \\
3.7963 E+00 \\
3.0286 E+00 \\
2.3491 E+00 \\
1.8503 E+00 \\
1.3243 E+00 \\
1.0618 E+00 \\
8.1114 E-01 \\
5.5190 E-01 \\
3.0875 E-01 \\
3.0883 E-01 \\
2.4080 E-01 \\
1.7588 E-01 \\
1.2185 E-01 \\
8.5642 E-02 \\
5.7946 E-02 \\
3.7525 E-02 \\
2.3044 E-02 \\
1.3305 E-02 \\
9.7407 E-03 \\
2.4618 E-03 \\
4.3141 E-04 \\
5.3678 E-05 \\
5.0764 E-06 \\
4.7905 E-07 \\
3.2068 E-08 \\
1.3224 E-09 \\
4.2172 E-11 \\
1.0291 E-12 \\
1.9212 E-14 \\
2.6515 E-16 \\
1.1124 E-18 \\
1.0000 E+02\end{array}$ & $\begin{array}{l}5.4158 E-03 \\
3.9969 E-02 \\
1.1547 E-01 \\
3.9869 E-01 \\
1.5669 E+00 \\
3.2878 E+00 \\
1.0634 E+01 \\
4.5044 E+00 \\
6.5873 E+00 \\
6.7714 E+00 \\
6.1379 E+00 \\
5.6265 E+00 \\
6.2393 E+00 \\
5.6208 E+00 \\
5.6953 E+00 \\
5.9302 E+00 \\
5.2935 E+00 \\
4.0209 E+00 \\
3.9461 E+00 \\
3.8036 E+00 \\
3.1718 E+00 \\
2.5281 E+00 \\
2.0581 E+00 \\
1.5263 E+00 \\
1.2091 E+00 \\
9.2492 E-01 \\
6.9918 E-01 \\
4.9949 E-01 \\
3.5974 E-01 \\
2.5320 E-01 \\
1.7933 E-01 \\
1.2196 E-01 \\
8.4026 E-02 \\
5.7099 E-02 \\
3.8157 E-02 \\
2.4933 E-02 \\
1.5888 E-02 \\
1.5010 E-02 \\
5.5938 E-03 \\
1.8077 E-03 \\
5.2696 E-04 \\
1.4446 E-04 \\
3.2784 E-05 \\
7.9204 E-06 \\
1.5274 E-06 \\
2.8544 E-07 \\
4.8543 E-08 \\
7.7536 E-09 \\
9.0309 E-10 \\
4.3582 E-12 \\
1.0000 E+02\end{array}$ \\
\hline
\end{tabular}


TABLE B-X!1!

ZPR-6-6P CENTRRL FLUX SPCCTRA

\begin{tabular}{|c|c|c|c|c|}
\hline GROUP & $\begin{array}{r}\text { ENERGY } \\
\text { BOUNDRRY } \\
\text { IEVI }\end{array}$ & $\begin{array}{r}\text { DIFTUSION } \\
\text { THEORY } \\
\text { IN:CM2/SI }\end{array}$ & $\begin{array}{r}\text { TRANSPORT } \\
\text { THEORY } \\
\text { IN/CMZ } / S 1\end{array}$ & $\begin{array}{l}\text { DITFUSION } \\
\text { THEORY } \\
\text { (N/CM2/SI }\end{array}$ \\
\hline $\begin{array}{r}1 \\
2 \\
3 \\
4 \\
5 \\
6 \\
7 \\
8 \\
9 \\
10 \\
11 \\
12 \\
13 \\
14 \\
15 \\
16 \\
17 \\
18 \\
19 \\
20 \\
21 \\
22 \\
23 \\
24 \\
25 \\
26 \\
27 \\
28 \\
29 \\
30 \\
31 \\
32 \\
33 \\
34 \\
35 \\
36 \\
37 \\
38 \\
39 \\
40 \\
41 \\
42 \\
43 \\
44 \\
45 \\
46 \\
47 \\
48 \\
49 \\
50\end{array}$ & $\begin{array}{l}1.9971 E \cdot 07 \\
1.0000 E+07 \\
6.0653 E+06 \\
3.6788 E+06 \\
2.2313 E+06 \\
1.3534 E+06 \\
8.2085 E+05 \\
4.9787 E+05 \\
3.8774 E+05 \\
3.0197 E+05 \\
2.3518 E+05 \\
1.8316 E+05 \\
1.4264 E+05 \\
1.1109 E+05 \\
8.6517 E+04 \\
6.7379 E+04 \\
5.2475 E+04 \\
4.0868 E+04 \\
3.1828 E+04 \\
2.4788 E+04 \\
1.9305 E+04 \\
1.5034 E+04 \\
1.1709 E+04 \\
9.1188 E+03 \\
7.1017 E+03 \\
5.5308 E+03 \\
4.3074 E+03 \\
3.3546 E+03 \\
2.6126 E+03 \\
2.0347 E+03 \\
1.5846 E+03 \\
1.2341 E+03 \\
9.6112 E+02 \\
7.4852 E+02 \\
5.8295 E+02 \\
4.5400 E+02 \\
3.5358 E+02 \\
2.7536 E+02 \\
1.6702 E+02 \\
1.0130 E+02 \\
6.1442 E+01 \\
3.7267 E+01 \\
2.2603 E+01 \\
1.3710 E+01 \\
8.3153 E+00 \\
5.0435 E+00 \\
3.0590 E+00 \\
1.8554 E+00 \\
1.1254 E+00 \\
6.8256 E-01 \\
1.0000 E-05\end{array}$ & $\begin{array}{l}1.7292 E-02 \\
2.2892 E-01 \\
1.0858 E+00 \\
2.7687 E+00 \\
4.3236 E+00 \\
5.6968 E+00 \\
9.8037 E+00 \\
3.6708 E+00 \\
5.3477 E+00 \\
5.9233 E+00 \\
5.7516 E+00 \\
5.9930 E+00 \\
5.8373 E+00 \\
5.2704 E+00 \\
5.2027 E+00 \\
4.3083 E+00 \\
4.3201 E+00 \\
3.4599 E+00 \\
2.9359 E+00 \\
3.7140 E+00 \\
2.8010 E+00 \\
2.4501 E+00 \\
1.9084 E+00 \\
1.2568 E+00 \\
1.1641 E+00 \\
8.9552 E-01 \\
5.0692 E-01 \\
1.1625 E-01 \\
3.8066 E-01 \\
7.1325 E-01 \\
6.9147 E-01 \\
4.7218 E-01 \\
3.5598 E-01 \\
2.3850 E-01 \\
1.5282 E-01 \\
9.3671 E-02 \\
5.3501 E-02 \\
6.1400 E-02 \\
1.9656 E-02 \\
6.1531 E-03 \\
1.4187 E-03 \\
2.7077 E-04 \\
4.5418 E-05 \\
8.0758 E-06 \\
9.6758 E-07 \\
3.1632 E-07 \\
1.3433 E-07 \\
4.3429 E-08 \\
7.1765 E-09 \\
4.7532 E-10 \\
1.0000 E+02\end{array}$ & $\begin{array}{l}1.7232 E-02 \\
2.2808 E-01 \\
1.0818 E+00 \\
2.7593 E+00 \\
4.3093 E+00 \\
5.6803 E+00 \\
9.7851 E+00 \\
3.6642 E+00 \\
5.3390 E+00 \\
5.9145 E+00 \\
5.7448 E+00 \\
5.9869 E+00 \\
5.8343 E+00 \\
5.2676 E+00 \\
5.205-4 E+00 \\
4.3112 E+00 \\
4.3244 E+00 \\
3.4617 E+00 \\
2.94 E-00+00 \\
3.7279 E+00 \\
2.8127 E+00 \\
2.4608 E+00 \\
1.9172 E+00 \\
1.2629 E+00 \\
1.1699 E+00 \\
9.0000 E-01 \\
5.0958 E-01 \\
1.1686 E-01 \\
3.8238 E-01 \\
7.1728 E-01 \\
6.9550 E-01 \\
4.7512 E-01 \\
3.5835 E-01 \\
2.4019 E-01 \\
1.5394 E-01 \\
9.4361 E-02 \\
5.3911 E-02 \\
6.1931 E-02 \\
1.9834 E-02 \\
6.2131 E-03 \\
1.4328 E-03 \\
2.7358 E-04 \\
4.5915 E-05 \\
8.1661 E-06 \\
9.7894 E-07 \\
3.2002 E-07 \\
1.3589 E-07 \\
4.3929 E-08 \\
7.2592 E-09 \\
4.8084 E-10 \\
1.0000 E+02\end{array}$ & $\begin{array}{l}1.7332 E-02 \\
2.2949 E-01 \\
1.0887 E+00 \\
2.7755 E+00 \\
4.3322 E+00 \\
5.7061 E+00 \\
9.8063 E+00 \\
3.6713 E+00 \\
5.3451 E+00 \\
5.9173 E+00 \\
5.7440 E+00 \\
5.9834 E+00 \\
5.8271 E+00 \\
5.2611 E+00 \\
5.1939 E+00 \\
4.3018 E+00 \\
4.3149 E+00 \\
3.4568 E+00 \\
2.9337 E+00 \\
3.7134 E+00 \\
2.8026 E+00 \\
2.4536 E+00 \\
1.9130 E+00 \\
1.2604 E+00 \\
1.1678 E+00 \\
8.9871 E-01 \\
5.0880 E-01 \\
1.1668 E-01 \\
3.8209 E-01 \\
7.1630 E-01 \\
6.9501 E-01 \\
4.7496 E-01 \\
3.5841 E-01 \\
2.4032 E-01 \\
1.5404 E-01 \\
9.4401 E-02 \\
5.3939 E-02 \\
6.1917 E-02 \\
1.9788 E-02 \\
6.1892 E-03 \\
1.4192 E-03 \\
2.7036 E-04 \\
4.5523 E-05 \\
8.1205 E-06 \\
9.0149 E-07 \\
2.9576 E-07 \\
1.2721 E-07 \\
4.1569 E-08 \\
6.9049 E-09 \\
4.5702 E-10 \\
1.0000 E+02\end{array}$ \\
\hline
\end{tabular}


TABLE $8-X L\|\|$

ZPR-G-6A EDGE FLUX SPECTRA

\begin{tabular}{|c|c|c|c|c|}
\hline GROUP & $\begin{array}{r}\text { ENERGY } \\
\text { BOUNORRY } \\
\text { (EV) }\end{array}$ & $\begin{array}{r}1-0 \\
\text { OITFUSION } \\
\text { THEORY } \\
\text { IN/CMZ } / S I\end{array}$ & $\begin{array}{r}1-0 \\
\text { TRANSPORT } \\
\text { THEORY } \\
\text { IN/CMZ/SI }\end{array}$ & $\begin{array}{r}2-0 \\
\text { OIFFUSION } \\
\text { THEORY } \\
\text { IN/CM2/SI }\end{array}$ \\
\hline $\begin{array}{l}1 \\
2 \\
3 \\
4 \\
5 \\
6 \\
7 \\
8 \\
9 \\
10 \\
11 \\
12 \\
13 \\
14 \\
15 \\
16 \\
17 \\
18 \\
19 \\
20 \\
21 \\
22 \\
23 \\
24 \\
25 \\
26 \\
27 \\
28 \\
29 \\
30 \\
31 \\
32 \\
33 \\
34 \\
35 \\
36 \\
37 \\
38 \\
39 \\
40 \\
41 \\
42 \\
43 \\
44 \\
45 \\
46 \\
47 \\
48 \\
49 \\
50\end{array}$ & $\begin{array}{l}1.9971 E+07 \\
1.0000 E+07 \\
6.0653 E+06 \\
3.6788 E+06 \\
2.2313 E+06 \\
1.3534 E+06 \\
8.2085 E+05 \\
4.9787 E+05 \\
3.8774 E+05 \\
3.0197 E+05 \\
2.3518 E+05 \\
1.8316 E+05 \\
1.4264 E+05 \\
1.1109 E+05 \\
8.6517 E+04 \\
6.7379 E+04 \\
5.2475 E+04 \\
4.0868 E+04 \\
3.1828 E+04 \\
2.4788 E+04 \\
1.9305 E+04 \\
1.5034 E+04 \\
1.1709 E+04 \\
9.1188 E+03 \\
7.1017 E+03 \\
5.5308 E+03 \\
4.3074 E+03 \\
3.3546 E+03 \\
2.6126 E+03 \\
2.0347 E+03 \\
1.5846 E+03 \\
1.2341 E+03 \\
9.6112 E+02 \\
7.4852 E+02 \\
5.8295 E+02 \\
4.5400 E+02 \\
3.5358 E+02 \\
2.7536 E+02 \\
1.6702 E+02 \\
1.0130 E+02 \\
6.1442 E+01 \\
3.7267 E+01 \\
2.2603 E+01 \\
1.3710 E+01 \\
8.3153 E+00 \\
5.0435 E+00 \\
3.0590 E+00 \\
1.8554 E+0 C \\
1.1254 E+00 \\
6.8256 E-01 \\
1.0000 E-05\end{array}$ & $\begin{array}{l}2.3366 E-03 \\
2.5166 E-02 \\
9.6048 E-02 \\
2.0123 E-01 \\
3.3843 E-01 \\
1.2071 E+00 \\
7.3941 E+00 \\
5.1534 E+00 \\
6.5082 E+00 \\
7.0447 E+00 \\
6.9035 E+00 \\
6.7170 E+00 \\
6.8617 E+00 \\
6.8824 E+00 \\
7.8335 E+00 \\
7.1679 E+00 \\
7.4000 E+00 \\
5.7688 E+00 \\
3.6693 E+00 \\
4.8955 E+00 \\
2.6851 E+00 \\
1.8436 E+00 \\
1.1771 E+00 \\
5.6057 E-01 \\
4.3134 E-01 \\
2.8446 E-01 \\
2.4100 E-01 \\
1.0676 E-01 \\
5.2921 E-02 \\
1.7228 E-01 \\
1.1943 E-01 \\
5.7520 E-02 \\
6.7917 E-02 \\
3.8556 E-02 \\
3.0364 E-0 E \\
1.6649 E-02 \\
8.9063 E-03 \\
2.9578 E-02 \\
4.2714 E-03 \\
9.3145 E-04 \\
2.8164 E-04 \\
3.4726 E-05 \\
1.8910 E-06 \\
7.5785 E-07 \\
1.2645 E-08 \\
6.3492 E-09 \\
9.2790 E-09 \\
6.5893 E-09 \\
2.0051 E-09 \\
2.2370 E-10 \\
1.0000 E+02\end{array}$ & $\begin{array}{l}3.6533 E-03 \\
3.7720 E-02 \\
1.3907 E-01 \\
2.8458 E-01 \\
4.6714 E-01 \\
1.4813 E+00 \\
7.7513 E+00 \\
5.2907 E+00 \\
6.6015 E+00 \\
7.1126 E+00 \\
6.9572 E+00 \\
6.7635 E+00 \\
6.8965 E+00 \\
6.9286 E+00 \\
7.8556 E+00 \\
7.2118 E+00 \\
7.3153 E+00 \\
5.6257 E+00 \\
3.5171 E+00 \\
4.6251 E+00 \\
2.5275 E+00 \\
1.7262 E+00 \\
1.0934 E+00 \\
5.2325 E-01 \\
3.9930 E-01 \\
2.6149 E-01 \\
2.1536 E-01 \\
9.5327 E-02 \\
4.4438 E-02 \\
5.6375 E-02 \\
5.2950 E-02 \\
2.7403 E-02 \\
3.4801 E-02 \\
2.1980 E-02 \\
2.0582 E-02 \\
1.2805 E-02 \\
6.9137 E-03 \\
1.1166 E-02 \\
2.1767 E-03 \\
4.5752 E-04 \\
1.8162 E-04 \\
2.3005 E-05 \\
1.2797 E-06 \\
6.5665 E-07 \\
1.0835 E-08 \\
6.4689 E-09 \\
9.9348 E-09 \\
7.0358 E-09 \\
2.1293 E-09 \\
2.3676 E-10 \\
1.0000 E+02\end{array}$ & $\begin{array}{l}3.8682 E-03 \\
4.3246 E-02 \\
1.6717 E-01 \\
3.5423 E-01 \\
5.8690 E-01 \\
1.8192 E+00 \\
8.6056 E+00 \\
5.7240 E+00 \\
6.9613 E+00 \\
7.3329 E+00 \\
7.0421 E+00 \\
6.7468 E+00 \\
6.7887 E+00 \\
6.6930 E+00 \\
7.4904 E+00 \\
6.7431 E+00 \\
6.7760 E+00 \\
5.0986 E+00 \\
3.2469 E+00 \\
4.3775 E+00 \\
2.3918 E+00 \\
1.6700 E+00 \\
1.0852 E+00 \\
5.2311 E-01 \\
4.1516 E-01 \\
2.8317 E-01 \\
2.4250 E-01 \\
1.1050 E-01 \\
5.5212 E-02 \\
1.8402 E-01 \\
1.3770 E-01 \\
6.8682 E-02 \\
8.1199 E-02 \\
4.6938 E-02 \\
3.6377 E-02 \\
1.9897 E-02 \\
1.0473 E-02 \\
3.0420 E-02 \\
4.6762 E-03 \\
1.0608 E-03 \\
3.1605 E-04 \\
3.8642 E-05 \\
2.2170 E-06 \\
8.1690 E-07 \\
1.4502 E-08 \\
9.8662 E-09 \\
1.4963 E-08 \\
9.5141 E-09 \\
2.5494 E-09 \\
2.3627 E-10 \\
1.0000 E+02\end{array}$ \\
\hline
\end{tabular}


TABLE $B-X L I V$

ZPR-6-6A CENTRAL ADJOINT SPECTRA

\begin{tabular}{|c|c|c|c|c|}
\hline SROUP & $\begin{array}{r}\text { ENERGY } \\
\text { BOUNDRRY } \\
\text { IEV I }\end{array}$ & $\begin{array}{r}1-D \\
\text { DIFFUSION } \\
\text { THEORY } \\
\text { IRHO/N/SI }\end{array}$ & $\begin{array}{r}1-0 \\
\text { TRANSPORT } \\
\text { THEORY } \\
\text { (RHO/N/SI }\end{array}$ & $\begin{array}{r}2-0 \\
\text { DIIFUSION } \\
\text { IHEORY } \\
\text { (RHO/N/S }\end{array}$ \\
\hline $\begin{array}{l}1 \\
2 \\
3 \\
4 \\
5 \\
6 \\
7 \\
8 \\
9 \\
10 \\
11 \\
12 \\
13 \\
14 \\
15 \\
16 \\
17 \\
18 \\
19 \\
20 \\
21 \\
22 \\
23 \\
21 \\
25 \\
26 \\
27 \\
28 \\
29 \\
30 \\
31 \\
32 \\
33 \\
34 \\
35 \\
36 \\
37 \\
38 \\
39 \\
40 \\
41 \\
42 \\
43 \\
44 \\
45 \\
46 \\
47 \\
48 \\
49 \\
50\end{array}$ & $\begin{array}{l}1.9971 E+07 \\
1.0000 E+07 \\
6.0653 E+06 \\
3.6788 E+06 \\
2.2313 E+06 \\
1.3534 E+06 \\
8.2085 E+05 \\
4.9787 E+05 \\
3.8774 E+05 \\
3.0197 E+05 \\
2.3518 E+05 \\
1.8316 E+05 \\
1.4264 E+05 \\
1.1109 E+05 \\
8.6517 E+04 \\
6.7379 E+04 \\
5.2475 E+04 \\
4.0868 E+04 \\
3.1828 E+04 \\
2.4788 E+04 \\
1.9305 E+04 \\
1.5034 E+04 \\
1.1709 E+04 \\
9.1188 E+03 \\
7.1017 E+03 \\
5.5308 E+03 \\
4.3074 E+03 \\
3.3546 E+03 \\
2.6126 E+03 \\
2.0347 E+03 \\
1.5846 E+03 \\
1.2341 E+03 \\
9.6112 E+02 \\
7.4852 E+02 \\
5.8295 E+02 \\
4.5400 E+02 \\
3.5358 E+02 \\
2.7536 E+02 \\
1.6702 E+02 \\
1.0130 E+02 \\
6.1442 E+01 \\
3.7267 E+01 \\
2.2603 E+01 \\
1.3710 E+01 \\
8.3153 E+00 \\
5.0435 E+00 \\
3.0590 E+00 \\
1.8554 E+0 E \\
1.1254 E+00 \\
6.8256 E-01 \\
1.0000 E-05\end{array}$ & $\begin{array}{l}1.3931 E+00 \\
1.2106 E+00 \\
1.0735 E+00 \\
1.0762 E+00 \\
1.0065 E+00 \\
9.2745 E-01 \\
9.2080 E-01 \\
9.3227 E-01 \\
9.2971 E-01 \\
9.2741 E-01 \\
9.2615 E-01 \\
9.2325 E-01 \\
9.1938 E-01 \\
9.1720 E-01 \\
9.1261 E-01 \\
9.0861 E-01 \\
9.0701 E-01 \\
9.1076 E-01 \\
9.1745 E-01 \\
9.2431 E-01 \\
9.4389 E-01 \\
9.6044 E-01 \\
9.7990 E-01 \\
1.0001 E+00 \\
1.0203 E+00 \\
1.0398 E+00 \\
1.0525 E+00 \\
1.0576 E+00 \\
1.0627 E+00 \\
1.0747 E+00 \\
1.0903 E+00 \\
1.0799 E+00 \\
1.1861 E+00 \\
1.2460 E+00 \\
1.2632 E+00 \\
1.2146 E+00 \\
1.2013 E+00 \\
1.2554 E+00 \\
1.1707 E+00 \\
1.2324 E+00 \\
1.4420 E+00 \\
1.1876 E+00 \\
1.0040 E+00 \\
1.2339 E+00 \\
4.4408 E-01 \\
1.1239 E+00 \\
1.3261 E+00 \\
1.5944 E+00 \\
1.8717 E+00 \\
1.8673 E+00 \\
5.4790 E+01\end{array}$ & $\begin{array}{l}1.3921 E+00 \\
1.2098 E+00 \\
1.0731 E+00 \\
1.0760 E+00 \\
1.0065 E+00 \\
9.2799 E-01 \\
9.2123 E-01 \\
9.3231 E-01 \\
9.2852 E-01 \\
9.2711 E-01 \\
9.2588 E-01 \\
9.2290 E-01 \\
9.1906 E-01 \\
9.1673 E-01 \\
9.1238 E-01 \\
9.0781 E-01 \\
9.0623 E-01 \\
9.1012 E-01 \\
9.1704 E-01 \\
9.2155 E-01 \\
9.4104 E-01 \\
9.5732 E-01 \\
9.7671 E-01 \\
9.9692 E-01 \\
1.0169 E+00 \\
1.0364 E+00 \\
1.0491 E+00 \\
1.0542 E+00 \\
1.0593 E+00 \\
1.0711 E+00 \\
1.0865 E+00 \\
1.0761 E+00 \\
1.1818 E+00 \\
1.2413 E+00 \\
1.2584 E+00 \\
1.2102 E+00 \\
1.1972 E+00 \\
1.2507 E+00 \\
1.1663 E+00 \\
1.2275 E+00 \\
1.4359 E+00 \\
1.1828 E+00 \\
9.9997 E-01 \\
1.2286 E+00 \\
4.4226 E-01 \\
1.1189 E+00 \\
1.3201 E+00 \\
1.5872 E+00 \\
1.8631 E+00 \\
1.8587 E+00 \\
5.4647 E+01\end{array}$ & $\begin{array}{l}1.3924 E+00 \\
1.2104 E+00 \\
1.0736 E+00 \\
1.0764 E+00 \\
1.0068 E+00 \\
9.2760 E-01 \\
9.2060 E-01 \\
9.3160 E-01 \\
9.2797 E-01 \\
9.2664 E-01 \\
9.2537 E-01 \\
9.2247 E-01 \\
9.1858 E-01 \\
9.1637 E-01 \\
9.1173 E-01 \\
9.0766 E-01 \\
9.0596 E-01 \\
9.0959 E-01 \\
9.1615 E-01 \\
9.2290 E-01 \\
9.4231 E-01 \\
9.5872 E-01 \\
9.7803 E-01 \\
9.9814 E-01 \\
1.0182 E+00 \\
1.0375 E+00 \\
1.0500 E+00 \\
1.0551 E+00 \\
1.0601 E+00 \\
1.0721 E+00 \\
1.0874 E+00 \\
1.0768 E+00 \\
1.1824 E+00 \\
1.2417 E+00 \\
1.2581 E+00 \\
1.2091 E+00 \\
1.1963 E+00 \\
1.2493 E+00 \\
1.1624 E+00 \\
1.2230 E+00 \\
1.4291 E+00 \\
1.1819 E+00 \\
1.0043 E+00 \\
1.2284 E+00 \\
4.0993 E-01 \\
1.1170 E+00 \\
1.3249 E+00 \\
1.5932 E+[10 \\
1.8706 E+00 \\
1.8655 E+00 \\
5.4632 E+01\end{array}$ \\
\hline
\end{tabular}


TAELE B-XLV

SNEAK - 7A CENTRAL FLUXX SPECTRA

\begin{tabular}{|c|c|c|c|c|}
\hline GROUP & $\begin{array}{r}\text { ENERGY } \\
\text { BOUNDRRY } \\
\text { [EVI }\end{array}$ & $\begin{array}{r}1-D \\
\text { DIFFUSION } \\
\text { THEORY } \\
\text { IN/CM2/SI }\end{array}$ & $\begin{array}{r}1-0 \\
\text { TRANSPORT } \\
\text { IHEORY } \\
|\mathrm{N} / \mathrm{CM} / \mathrm{SI}|\end{array}$ & $\begin{array}{r}2-0 \\
\text { OIFFUSION } \\
\text { THEORY } \\
\text { IN/CM2/SI }\end{array}$ \\
\hline $\begin{array}{l}1 \\
2 \\
3 \\
4 \\
5 \\
6 \\
7 \\
8 \\
9 \\
10 \\
11 \\
12 \\
13 \\
14 \\
15 \\
16 \\
17 \\
18 \\
19 \\
20 \\
21 \\
22 \\
23 \\
24 \\
25 \\
26 \\
27 \\
28 \\
29 \\
30 \\
31 \\
32 \\
33 \\
34 \\
35 \\
36 \\
37 \\
38 \\
39 \\
40 \\
41 \\
42 \\
43 \\
44 \\
45 \\
46 \\
47 \\
48 \\
49 \\
50\end{array}$ & $\begin{array}{l}1.9971 E+07 \\
1.0000 E+07 \\
6.0653 E+06 \\
3.6788 E+06 \\
2.2313 E+06 \\
1.35+4 E+06 \\
8.2085 E+05 \\
4.9787 E+05 \\
3.8774 E+05 \\
3.0197 E+05 \\
2.3518 E+05 \\
1.8316 E+05 \\
1.4264 E+05 \\
1.1109 E+05 \\
8.6517 E+04 \\
6.7379 E+04 \\
5.2475 E+04 \\
4.0868 E+04 \\
3.1828 E+04 \\
2.4788 E+04 \\
1.9305 E+04 \\
1.5034 E+04 \\
1.1709 E+04 \\
9.1188 E+03 \\
7.1017 E+03 \\
5.5308 E+03 \\
4.3074 E+03 \\
3.3546 E+03 \\
2.6126 E+03 \\
2.0347 E+03 \\
1.5846 E+03 \\
1.2341 E+03 \\
9.6112 E+02 \\
7.4852 E+02 \\
5.8295 E+02 \\
4.5400 E+02 \\
3.5358 E+02 \\
2.7536 E+02 \\
1.6702 E+02 \\
1.0130 E+02 \\
6.1442 E+01 \\
3.7267 E+01 \\
2.2603 E+01 \\
1.3710 E+01 \\
8.3153 E+00 \\
5.0435 E+00 \\
3.0590 E+00 \\
1.8554 E+00 \\
1.1254 E+00 \\
6.8256 E-01 \\
1.0000 E-05\end{array}$ & $\begin{array}{l}4.1850 E-02 \\
4.5370 E-01 \\
2.2295 E+00 \\
5.0662 E+00 \\
6.8340 E+00 \\
8.5730 E+00 \\
1.0331 E+01 \\
4.1180 E+00 \\
5.0349 E+00 \\
5.2318 E+00 \\
5.0214 E+00 \\
4.7956 E+00 \\
4.5194 E+00 \\
4.1949 E+00 \\
3.9530 E+00 \\
3.6773 E+00 \\
3.3776 E+00 \\
2.9139 E+00 \\
2.6124 E+00 \\
2.6031 E+00 \\
2.2384 E+00 \\
2.0164 E+00 \\
1.7325 E+00 \\
1.4354 E+00 \\
1.2971 E+00 \\
1.1253 E+00 \\
9.7536 E-01 \\
8.1530 E-01 \\
6.6471 E-01 \\
5.5480 E-01 \\
4.4425 E-01 \\
3.2436 E-01 \\
2.4769 E-01 \\
1.8646 E-01 \\
1.2391 E-01 \\
9.0193 E-02 \\
5.7335 E-02 \\
5.9313 E-02 \\
2.1561 E-02 \\
5.4740 E-03 \\
1.3618 E-03 \\
6.2187 E-04 \\
1.0424 E-04 \\
2.4509 E-05 \\
4.0668 E-06 \\
2.2303 E-06 \\
1.1097 E-06 \\
2.0382 E-07 \\
1.1871 E-08 \\
3.4205 E-10 \\
1.0000 E+02\end{array}$ & $\begin{array}{l}4.2158 E-02 \\
4.5900 E-01 \\
2.2272 E+00 \\
5.0591 E+00 \\
6.8163 E+00 \\
8.5591 E+00 \\
1.0333 E+01 \\
4.1208 E+00 \\
5.0378 E+00 \\
5.2354 E+00 \\
5.0255 E+00 \\
4.7993 E+00 \\
4.5229 E+00 \\
4.1970 E+00 \\
3.9555 E+00 \\
3.6776 E+00 \\
3.3767 E+00 \\
2.9114 E+00 \\
2.6159 E+00 \\
2.6052 E+00 \\
2.2399 E+00 \\
2.0169 E+00 \\
1.7324 E+00 \\
1.4351 E+00 \\
1.2966 E+00 \\
1.1246 E+00 \\
9.7506 E-01 \\
8.1472 E-01 \\
6.6410 E-01 \\
5.5177 E-01 \\
4.4533 E-01 \\
3.2553 E-01 \\
2.4896 E-01 \\
1.8774 E-01 \\
1.2497 E-01 \\
9.1059 E-02 \\
5.7970 E-02 \\
6.0238 E-02 \\
2.1980 E-02 \\
5.6005 E-03 \\
1.3975 E-03 \\
6.4167 E-04 \\
1.0795 E-04 \\
2.5464 E-05 \\
4.2410 E-06 \\
2.33: 0 E-06 \\
1.1622 E-06 \\
2.1390 E-07 \\
1.2474 E-08 \\
3.5951 E-10 \\
1.0000 E+02\end{array}$ & $\begin{array}{l}4.1613 E-02 \\
4.5517 E-01 \\
2.2165 E+00 \\
5.0376 E+00 \\
6.7983 E+00 \\
8.5326 E+00 \\
1.0299 E+01 \\
4.1060 E+00 \\
5.0232 E+00 \\
5.2238 E+00 \\
5.0175 E+00 \\
4.7953 E+00 \\
4.5224 E+00 \\
4.2004 E+00 \\
3.9608 E+00 \\
3.6873 E+00 \\
3.3895 E+00 \\
2.9260 E+00 \\
2.6246 E+00 \\
2.6172 E+00 \\
2.2520 E+00 \\
2.0299 E+00 \\
1.7452 E+00 \\
1.4464 E+00 \\
1.3076 E+00 \\
1.1348 E+00 \\
9.8379 E-01 \\
8.2249 E-01 \\
6.7065 E-01 \\
5.3577 E-01 \\
4.4829 E-01 \\
3.2731 E-01 \\
2.4994 E-01 \\
1.8315 E-01 \\
1.2503 E-01 \\
9.1002 E-02 \\
5.7845 E-02 \\
5.9827 E-02 \\
2.1743 E-02 \\
5.5189 E-03 \\
1.3726 E-03 \\
6.2626 E-04 \\
1.0494 E-04 \\
2.4653 E-05 \\
4.0879 E-06 \\
2.2317 E-06 \\
1.1041 E-06 \\
2.0239 E-07 \\
1.1782 E-08 \\
3.3949 E-10 \\
1.0000 E+02\end{array}$ \\
\hline
\end{tabular}


TABLE $B-X L V I$

SNEAK-7A EOGE FLUX SPECTRA

\begin{tabular}{|c|c|c|c|c|}
\hline GROUP & $\begin{array}{r}\text { ENERGY } \\
\text { BOUNDARY } \\
\text { IEVI }\end{array}$ & $\begin{array}{r}1-0 \\
\text { DIFFUS!ON } \\
\text { THEORY } \\
\text { IN } / \text { CMZ } / S I\end{array}$ & $\begin{array}{r}1-0 \\
\text { TRANSPORT } \\
\text { THEORY } \\
\text { IN/CM } 2 / S \mid\end{array}$ & $\begin{array}{r}2-0 \\
\text { DIFFUSION } \\
\text { THEORY } \\
\text { IN/CM2/SI }\end{array}$ \\
\hline $\begin{array}{l}1 \\
2 \\
3 \\
4 \\
5 \\
6 \\
7 \\
8 \\
9 \\
10 \\
11 \\
12 \\
13 \\
14 \\
15 \\
16 \\
17 \\
18 \\
19 \\
20 \\
21 \\
22 \\
23 \\
24 \\
25 \\
26 \\
27 \\
28 \\
29 \\
30 \\
31 \\
32 \\
33 \\
34 \\
35 \\
36 \\
37 \\
38 \\
39 \\
40 \\
41 \\
42 \\
43 \\
44 \\
43 \\
46 \\
47 \\
48 \\
49 \\
50\end{array}$ & $\begin{array}{l}1.9971 E+07 \\
1.0000 E+07 \\
6.0653 E+06 \\
3.6788 E+06 \\
2.2313 E+06 \\
1.3534 E+06 \\
8.20 B 5 E+05 \\
4.3787 E+05 \\
3.8774 E+05 \\
3.0197 E+05 \\
2.0518 E+05 \\
1.8316 E+05 \\
1.4264 E+05 \\
1.1109 E+05 \\
8.6517 E+04 \\
6.7379 E+04 \\
5.2475 E+04 \\
4.0868 E+04 \\
3.1828 E+04 \\
2.4788 E+04 \\
1.9305 E+04 \\
1.5034 E+04 \\
1.1709 E+04 \\
9.1188 E+03 \\
7.1017 E+03 \\
5.5308 E+03 \\
4.3074 E+03 \\
3.3546 E+03 \\
2.6126 E+03 \\
2.0347 E+03 \\
1.5846 E+03 \\
1.2341 E+03 \\
9.6112 E+02 \\
7.4852 E+02 \\
5.8295 E+02 \\
4.5400 E+02 \\
3.5358 E+02 \\
2.7536 E+02 \\
1.6702 E+02 \\
1.0130 E+02 \\
6.1442 E E+01 \\
3.7267 E+01 \\
2.2603 E+01 \\
1.3710 E+01 \\
8.3153 E+00 \\
5.0435 E+00 \\
3.0590 E+00 \\
1.8554 E+00 \\
1.1254 E+00 \\
6.8256 E-01 \\
1.0000 E-05\end{array}$ & $\begin{array}{l}7.9442 E-03 \\
7.8314 E-02 \\
2.7923 E-01 \\
5.4324 E-01 \\
8.4890 E-01 \\
2.4921 E+00 \\
9.8921 E+00 \\
6.6005 E+00 \\
7.8082 E+00 \\
8.0159 E+00 \\
7.5549 E+00 \\
7.0664 E+00 \\
6.8377 E+00 \\
6.7167 E+00 \\
6.8852 E+00 \\
6.3102 E+00 \\
6.1435 E+00 \\
4.4946 E+00 \\
2.8718 E+00 \\
3.2434 E+00 \\
1.6555 E+00 \\
1.2105 E+00 \\
8.1790 E-01 \\
4.1718 E-01 \\
3.0593 E-01 \\
1.9221 E-01 \\
1.6329 E-01 \\
9.3201 E-02 \\
7.0579 E-02 \\
1.6203 E-01 \\
8.6045 E-02 \\
3.7347 E-02 \\
3.6110 E-02 \\
1.9212 E-02 \\
1.3810 E-02 \\
7.5563 E-03 \\
4.2802 E-03 \\
1.3938 E-02 \\
1.8973 E-03 \\
4.3361 E-04 \\
9.4918 E-05 \\
1.1935 E-05 \\
6.6271 E-07 \\
3.0093 E-07 \\
5.0649 E-09 \\
1.3555 E-08 \\
4.0450 E-08 \\
2.1784 E-08 \\
4.4378 E-09 \\
3.0194 E-10 \\
1.0000 E+02\end{array}$ & $\begin{array}{l}9.8140 E-03 \\
9.3745 E-02 \\
3.3425 E-01 \\
6.4084 E-01 \\
9.8733 E-01 \\
2.7106 E+00 \\
9.9099 E+00 \\
6.5563 E+00 \\
7.7299 E+00 \\
7.9522 E+00 \\
7.5261 E+00 \\
7.0666 E+00 \\
6.8570 E+00 \\
6.7679 E+00 \\
6.9507 E+00 \\
6.4026 E+00 \\
5.1531 E+00 \\
4.4318 E+00 \\
2.8199 E+00 \\
3.1539 E+00 \\
1.6158 E+00 \\
1.1791 E+00 \\
7.8943 E-01 \\
4.0428 E-01 \\
2.9389 E-01 \\
1.8385 E-01 \\
1.5064 E-01 \\
8.5937 E-02 \\
5.3403 E-02 \\
5.9579 E-02 \\
4.5262 E-02 \\
2.1452 E-02 \\
2.1988 E-02 \\
1.2850 E-02 \\
1.0733 E-02 \\
6.6455 E-03 \\
3.7479 E-03 \\
5.6814 E-03 \\
1.0743 E-03 \\
2.3595 E-04 \\
6.6280 E-05 \\
8.6833 E-06 \\
4.8078 E-00 \\
3.0564 E-07 \\
4.8071 E-09 \\
1.7558 E-08 \\
4.9472 E-08 \\
2.5868 E-08 \\
5.1962 E-09 \\
3.5305 E-10 \\
1.0000 E+02\end{array}$ & $\begin{array}{l}7.9817 E-03 \\
7.8681 E-02 \\
2.8104 E-01 \\
5.4821 E-01 \\
8.5960 E-01 \\
2.5330 E+00 \\
9.9462 E+00 \\
6.6260 E+00 \\
7.8209 E+00 \\
8.0187 E+00 \\
7.5529 E+00 \\
7.0594 E+00 \\
6.8254 E+00 \\
6.7009 E+00 \\
6.8619 E+00 \\
6.2845 E+00 \\
6.1088 E+00 \\
4.4598 E+00 \\
2.8514 E+00 \\
3.2191 E+00 \\
1.6474 E+00 \\
1.2093 E+00 \\
8.2176 E-01 \\
4.2143 E-01 \\
3.1111 E-01 \\
1.9698 E-01 \\
1.6811 E-01 \\
9.7211 E-02 \\
7.4818 E-02 \\
1.7149 E-01 \\
9.1731 E-02 \\
3.9989 E-02 \\
3.8730 E-02 \\
2.0678 E-02 \\
1.4870 E-02 \\
8.1462 E-03 \\
4.6121 E-03 \\
1.4502 E-02 \\
1.9865 E-03 \\
4.5495 E-04 \\
1.0069 E-04 \\
1.2634 E-05 \\
6.9944 E-07 \\
2.9785 E-07 \\
5.0038 E-09 \\
1.2112 E-08 \\
3.4443 E-08 \\
1.8354 E-08 \\
3.7191 E-09 \\
2.5307 E-10 \\
1.0000 E+02\end{array}$ \\
\hline
\end{tabular}


TRBLE B-XLVII

SNEAK-7A CENTRAL ADJOINT SPECTRA

\begin{tabular}{|c|c|c|c|c|}
\hline GROUP & $\begin{array}{r}\text { ENERGY } \\
\text { BOUNDRRY } \\
\text { (EV) }\end{array}$ & $\begin{array}{r}1-0 \\
\text { DIFFUSION } \\
\text { THEORY } \\
\text { (RHO/N/S) }\end{array}$ & $\begin{array}{r}1-D \\
\text { TRANSPORT } \\
\text { THEORY } \\
(R H O / N / S)\end{array}$ & $\begin{array}{r}2-D \\
\text { OIFFUSION } \\
\text { THEORY } \\
\text { (RHO/N/S }\end{array}$ \\
\hline $\begin{array}{l}1 \\
2 \\
3 \\
4 \\
5 \\
6 \\
7 \\
8 \\
9 \\
10 \\
11 \\
12 \\
13 \\
14 \\
15 \\
16 \\
17 \\
18 \\
19 \\
20 \\
21 \\
22 \\
23 \\
24 \\
25 \\
26 \\
27 \\
28 \\
29 \\
30 \\
31 \\
32 \\
33 \\
34 \\
35 \\
36 \\
37 \\
38 \\
39 \\
40 \\
41 \\
42 \\
43 \\
44 \\
45 \\
46 \\
17 \\
48 \\
49 \\
50\end{array}$ & $\begin{array}{l}1.9971 E+07 \\
1.0000 E+07 \\
6.0653 E+06 \\
3.6788 E+06 \\
2.2313 E+06 \\
1.3534 E+06 \\
8.2085 E+05 \\
4.9787 E+05 \\
3.8774 E+05 \\
3.0197 E+05 \\
2.3518 E+05 \\
1.8316 E+05 \\
1.4264 E+05 \\
1.1109 E+05 \\
8.6517 E+04 \\
6.7379 E+04 \\
5.2475 E+04 \\
4.0868 E+04 \\
3.1828 E+04 \\
2.4788 E+04 \\
1.9305 E+04 \\
1.5034 E+04 \\
1.1709 E+04 \\
9.1188 E+03 \\
7.1017 E+03 \\
5.5308 E+03 \\
4.3074 E+03 \\
3.3546 E+03 \\
2.612 .5 E+03 \\
2.0347 E+03 \\
1.5846 E+03 \\
1.2341 E+03 \\
9.6112 E+02 \\
7.4852 E+02 \\
5.8295 E+02 \\
4.5400 E+02 \\
3.5358 E+02 \\
2.7536 E+02 \\
1.6702 E+02 \\
1.0130 E+02 \\
6.1442 E+01 \\
3.7267 E+01 \\
2.2603 E+01 \\
1.3710 E+01 \\
8.3153 E+00 \\
5.0435 E+00 \\
3.0590 E+00 \\
1.8554 E+00 \\
1.1254 E+00 \\
6.8256 E-01 \\
1.0000 E-05\end{array}$ & $\begin{array}{l}1.3874 E+00 \\
1.1743 E+00 \\
1.0592 E+00 \\
1.0641 E+00 \\
1.0037 E+00 \\
9.4138 E-01 \\
9.2537 E-01 \\
9.3851 E-01 \\
9.2723 E-01 \\
9.2175 E-01 \\
9.1953 E-01 \\
9.1593 E-01 \\
9.1106 E-01 \\
9.0521 E-01 \\
8.9549 E-01 \\
8.8905 E-01 \\
8.8449 E-01 \\
8.8332 E-01 \\
8.8256 E-01 \\
8.7976 E-01 \\
8.8922 E-01 \\
8.9713 E-01 \\
9.0923 E-01 \\
9.2460 E-01 \\
9.3246 E-01 \\
9.5327 E-01 \\
9.7743 E-01 \\
9.9641 E-01 \\
1.0067 E+00 \\
1.0503 E+00 \\
1.1090 E+00 \\
1.1126 E+00 \\
1.1450 E+00 \\
1.1833 E+00 \\
1.2908 E+00 \\
1.2204 E+00 \\
1.2283 E+00 \\
1.2805 E+00 \\
1.2953 E+00 \\
1.5530 E+00 \\
1.2572 E+00 \\
1.0940 E+00 \\
1.3886 E+00 \\
1.5887 E+00 \\
8.8277 E-01 \\
1.5087 E+00 \\
1.3294 E+00 \\
7.9925 E-01 \\
6.1597 E-01 \\
1.9178 E+00 \\
5.3648 E+01\end{array}$ & $\begin{array}{l}1.4095 E+00 \\
1.1909 E+00 \\
1.0676 E+00 \\
1.0683 E+00 \\
1.0031 E+00 \\
9.3761 E-01 \\
9.1945 E-01 \\
9.3055 E-01 \\
9.1862 E-01 \\
9.1317 E-01 \\
9.1104 E-01 \\
9.0754 E-01 \\
9.0285 E-01 \\
8.9711 E-01 \\
8.8878 E-01 \\
8.8124 E-01 \\
8.7714 E-01 \\
8.7627 E-01 \\
8.7589 E-01 \\
8.7087 E-01 \\
8.8017 E-01 \\
8.8779 E-01 \\
8.9974 E-01 \\
9.1494 E-01 \\
9.2275 E-01 \\
9.4338 E-01 \\
9.6736 E-01 \\
9.8612 E-01 \\
9.9667 E-01 \\
1.0397 E+00 \\
1.0969 E+00 \\
1.1006 E+00 \\
1.1324 E+00 \\
1.1701 E+00 \\
1.2759 E+00 \\
1.2064 E+00 \\
1.2149 E+00 \\
1.2665 E+00 \\
1.2807 E+00 \\
1.5341 E+00 \\
1.2423 E+00 \\
1.0821 E+00 \\
1.3719 E+00 \\
1.5687 E+00 \\
8.7157 E-01 \\
1.4863 E+00 \\
1.3101 E+00 \\
7.8835 E-01 \\
6.0744 E-01 \\
1.8894 E+00 \\
5.3183 E+01\end{array}$ & $\begin{array}{l}1.3877 E+00 \\
1.1747 E+00 \\
1.0594 E+00 \\
1.0641 E+00 \\
1.0036 E+00 \\
9.4134 E-01 \\
9.2554 E-01 \\
9.3851 E-01 \\
9.2726 E-01 \\
9.2170 E-01 \\
9.1931 E-01 \\
9.1554 E-01 \\
9.1049 E-01 \\
9.0444 E-01 \\
8.9557 E-01 \\
8.8795 E-01 \\
8.8320 E-01 \\
8.8181 E-01 \\
8.8087 E-01 \\
8.7795 E-01 \\
8.8709 E-01 \\
8.9475 E-01 \\
9.0655 E-01 \\
9.2160 E-01 \\
9.2930 E-01 \\
9.4986 E-01 \\
9.7377 E-01 \\
9.9253 E-01 \\
1.0027 E+00 \\
1.0460 E+00 \\
1.1043 E+00 \\
1.1078 E+00 \\
1.1399 E+00 \\
1.1780 E+00 \\
1.2848 E+00 \\
1.2147 E+00 \\
1.2224 E+00 \\
1.2743 E+00 \\
1.2890 E+00 \\
1.5452 E+00 \\
1.2510 E+00 \\
1.0889 E+00 \\
1.3815 E+00 \\
1.5806 E+00 \\
8.7830 E-01 \\
1.5013 E+00 \\
1.3229 E+00 \\
7.9514 E-01 \\
6.1274 E-01 \\
1.9077 E+00 \\
5.3486 E+01\end{array}$ \\
\hline
\end{tabular}


TABLE B-XL.VI]]

SNEAK-7B CENTRAL FLUX SPECTRA 
TABLE $B-X L I X$

SNEAK-7B EDGE FLUX SPECTRA

\begin{tabular}{|c|c|c|c|c|}
\hline GROUP & $\begin{array}{r}\text { ENERGY } \\
\text { BOUNDFRY } \\
\text { (EV) }\end{array}$ & $\begin{array}{r}1-D \\
\text { OIFFUSION } \\
\text { THEORY } \\
\text { (N/CMZ/S ) }\end{array}$ & $\begin{array}{r}1-0 \\
\text { TRANSPORT } \\
\text { THEORY } \\
\text { (N/CM } 2 / S \text { ) }\end{array}$ & $\begin{array}{r}2-0 \\
\text { DIFFUSION } \\
\text { THEORY } \\
\text { IN/CM } 2 / S I\end{array}$ \\
\hline $\begin{array}{r}1 \\
2 \\
3 \\
4 \\
5 \\
6 \\
7 \\
8 \\
9 \\
10 \\
11 \\
12 \\
13 \\
14 \\
15 \\
16 \\
17 \\
18 \\
19 \\
20 \\
21 \\
22 \\
23 \\
24 \\
25 \\
26 \\
27 \\
28 \\
29 \\
30 \\
31 \\
32 \\
33 \\
34 \\
35 \\
36 \\
37 \\
38 \\
39 \\
40 \\
41 \\
42 \\
43 \\
44 \\
45 \\
46 \\
47 \\
48 \\
49 \\
50\end{array}$ & $\begin{array}{l}1.9971 E+07 \\
1.0000 E+07 \\
6.0653 E+06 \\
3.6788 E+06 \\
2.2313 E+06 \\
1.3534 E+06 \\
8.2085 E+05 \\
4.9787 E+05 \\
3.8774 E+05 \\
3.0197 E+05 \\
2.3518 E+05 \\
1.8316 E+05 \\
1.4264 E+05 \\
1.1109 E+05 \\
8.6517 E+04 \\
6.7379 E+04 \\
5.2475 E+04 \\
4.0868 E+04 \\
3.1828 E+04 \\
2.4788 E+04 \\
1.9305 E+04 \\
1.5034 E+04 \\
1.1709 E+04 \\
9.1188 E+03 \\
7.1017 E+03 \\
5.5308 E+03 \\
4.3074 E+03 \\
3.3546 E+03 \\
2.6126 E+03 \\
2.0347 E+03 \\
1.5846 E+03 \\
1.2341 E+03 \\
9.6112 E+02 \\
7.4852 E+02 \\
5.8295 E+02 \\
4.5400 E+02 \\
3.5358 E+02 \\
2.7536 E+02 \\
1.6702 E+02 \\
1.0130 E+02 \\
6.1442 E+01 \\
3.7267 E+01 \\
2.2603 E+01 \\
1.3710 E+01 \\
8.3153 E+00 \\
5.0435 E+00 \\
3.0590 E+00 \\
1.8554 E+00 \\
1.1254 E+00 \\
6.8256 E-01 \\
1.0000 E-05\end{array}$ & $\begin{array}{l}7.3510 E-03 \\
7.3678 E-02 \\
2.6166 E-01 \\
5.0901 E-01 \\
7.8362 E-01 \\
2.2354 E+00 \\
9.6590 E+00 \\
6.3203 E+00 \\
7.4766 E+00 \\
7.7161 E+00 \\
7.3216 E+00 \\
E .9025 E+00 \\
6.7845 E+00 \\
6.7035 E+00 \\
7.0291 E+00 \\
6.5123 E+00 \\
6.5130 E+00 \\
4.8323 E+00 \\
3.0713 E+00 \\
3.5965 E+00 \\
1.8440 E+00 \\
1.3527 E+00 \\
9.2236 E-01 \\
4.6009 E-01 \\
3.3382 E-01 \\
2.0399 E-01 \\
1.6982 E-01 \\
9.2839 E-02 \\
6.4551 E-02 \\
1.1901 E-01 \\
5.8894 E-02 \\
2.3838 E-02 \\
2.0045 E-02 \\
9.5976 E-03 \\
6.1139 E-03 \\
3.0609 E-03 \\
1.5842 E-03 \\
3.8301 E-03 \\
4.7184 E-04 \\
8.8541 E-05 \\
1.5267 E-05 \\
1.6118 E-06 \\
7.8903 E-08 \\
1.6155 E-08 \\
2.6317 E-10 \\
1.7462 E-10 \\
2.9654 E-10 \\
1.4066 E-10 \\
2.7353 E-11 \\
1.8484 E-12 \\
1.0000 E+02\end{array}$ & $\begin{array}{l}9.0492 E-03 \\
8.8906 E-02 \\
3.1224 E-01 \\
5.9891 E-01 \\
9.0435 E-01 \\
2.4433 E+00 \\
9.7537 E+00 \\
6.3044 E+00 \\
7.4228 E+00 \\
7.6719 E+00 \\
7.3048 E+00 \\
6.9085 E+00 \\
6.8050 E+00 \\
6.7517 E+00 \\
7.0848 E+00 \\
6.5955 E+00 \\
6.4996 E+00 \\
4.7432 E+00 \\
2.9945 E+00 \\
3.4665 E+00 \\
1.7825 E+00 \\
1.3044 E+00 \\
8.8125 E-01 \\
4.4164 E-01 \\
3.1800 E-01 \\
1.9363 E-01 \\
1.5628 E-01 \\
8.5449 E-02 \\
4.9556 E-02 \\
4.7965 E-02 \\
3.2251 E-02 \\
1.4015 E-02 \\
1.2309 E-02 \\
6.4221 E-03 \\
4.6805 E-03 \\
2.6224 E-03 \\
1.3548 E-03 \\
1.7258 E-03 \\
2.8287 E-04 \\
5.0889 E-05 \\
1.0763 E-05 \\
1.1704 E-06 \\
5.7831 E-08 \\
1.4598 E-08 \\
2.2473 E-10 \\
2.0090 E-10 \\
3.4664 E-10 \\
1.6107 E-10 \\
3.0966 E-11 \\
2.0897 E-12 \\
1.0000 E+02\end{array}$ & $\begin{array}{l}7.3590 E-03 \\
7.3740 E-02 \\
2.6206 E-01 \\
5.1060 E-01 \\
7.8805 E-01 \\
2.2590 E+00 \\
9.7099 E+00 \\
6.3481 E+00 \\
7.4979 E+00 \\
7.7309 E+00 \\
7.3323 E+00 \\
6.9089 E+00 \\
6.7866 E+00 \\
6.7024 E+00 \\
7.0207 E+00 \\
6.4977 E+00 \\
6.4858 E+00 \\
4.8005 E+00 \\
3.0508 E+00 \\
3.5635 E+00 \\
1.8275 E+00 \\
1.3414 E+00 \\
9.1586 E-01 \\
4.5788 E-01 \\
3.3296 E-01 \\
2.0407 E-01 \\
1.7036 E-01 \\
9.3637 E-02 \\
5.5647 E-02 \\
1.2171 E-01 \\
6.0692 E-02 \\
2.4679 E-02 \\
2.0851 E-02 \\
1.0035 E-02 \\
6.4188 E-03 \\
3.2251 E-03 \\
1.6725 E-03 \\
3.9938 E-03 \\
4.9457 E-04 \\
9.2773 E-05 \\
1.6110 E-05 \\
1.7028 E-06 \\
8.3517 E-08 \\
1.7330 E-08 \\
2.8269 E-10 \\
1.9182 E-10 \\
3.2519 E-10 \\
1.5306 E-10 \\
2.9612 E-11 \\
2.0007 E-12 \\
1.0000 E+02\end{array}$ \\
\hline
\end{tabular}


TABLE B-L

SNEAK-7B CENTRAL ADJOINT SPECTRA

\begin{tabular}{|c|c|c|c|c|}
\hline GROUP & $\begin{array}{r}\text { ENERGY } \\
\text { BOUNDRRY } \\
\text { (EV) }\end{array}$ & $\begin{array}{r}\text { !-D } \\
\text { DIFFUSION } \\
\text { THEORY } \\
\text { IRHO/N/SI }\end{array}$ & $\begin{array}{r}1-0 \\
\text { TRANSPORT } \\
\text { THEORY } \\
\text { IRHO/N/SI }\end{array}$ & $\begin{array}{r}2-0 \\
\text { DIFFUSION } \\
\text { THEORY } \\
\text { [RHO/N/SI }\end{array}$ \\
\hline $\begin{array}{r}1 \\
2 \\
3 \\
4 \\
5 \\
6 \\
7 \\
8 \\
9 \\
10 \\
11 \\
12 \\
13 \\
14 \\
15 \\
16 \\
17 \\
18 \\
19 \\
20 \\
21 \\
22 \\
23 \\
24 \\
25 \\
26 \\
27 \\
28 \\
29 \\
30 \\
31 \\
32 \\
33 \\
34 \\
35 \\
36 \\
37 \\
38 \\
39 \\
40 \\
41 \\
42 \\
43 \\
44 \\
45 \\
46 \\
47 \\
48 \\
49 \\
50\end{array}$ & $\begin{array}{l}1.9971 E+07 \\
1.0000 E+07 \\
6.0653 E+06 \\
3.6788 E+06 \\
2.2313 E+06 \\
1.3534 E+06 \\
8.2085 E+05 \\
4.9787 E+05 \\
3.8774 E+05 \\
3.0197 E+05 \\
2.3518 E+05 \\
1.8316 E+05 \\
1.4264 E+05 \\
1.1109 E+05 \\
8.6517 E+04 \\
6.7379 E+04 \\
5.2475 E+04 \\
4.0868 E+04 \\
3.1828 E+04 \\
2.4788 E+04 \\
1.9305 E+04 \\
1.5034 E+04 \\
1.1709 E+04 \\
9.1188 E+03 \\
7.1017 E+03 \\
5.5308 E+03 \\
4.3074 E+03 \\
3.3546 E+03 \\
2.6126 E+03 \\
2.0347 E+03 \\
1.5846 E+03 \\
1.2341 E+03 \\
9.6112 E+02 \\
7.4852 E+02 \\
5.8295 E+02 \\
4.5400 E+02 \\
3.5358 E+02 \\
2.7536 E+02 \\
1.6702 E+02 \\
1.0130 E+02 \\
6.1442 E+01 \\
3.7267 E+01 \\
2.2603 E+01 \\
1.3710 E+01 \\
8.3153 E+00 \\
5.0435 E+00 \\
3.0590 E+00 \\
1.8554 E+00 \\
1.1254 E+00 \\
6.8256 E-01 \\
1.0000 E-05\end{array}$ & $\begin{array}{l}1.6680 E+00 \\
1.3568 E+00 \\
1.1284 E+00 \\
1.1210 E+00 \\
1.0129 E+00 \\
8.8988 E-01 \\
8.6383 E-01 \\
8.5491 E-01 \\
8.3936 E-01 \\
8.2383 E-01 \\
8.0979 E-01 \\
7.9553 E-01 \\
7.7962 E-01 \\
7.6240 E-01 \\
7.4294 E-01 \\
7.2344 E-01 \\
7.0843 E-01 \\
6.9937 E-01 \\
6.9264 E-01 \\
6.8730 E-01 \\
6.9218 E-01 \\
6.9826 E-01 \\
7.0998 E-01 \\
7.2577 E-01 \\
7.3678 E-01 \\
7.6206 E-01 \\
7.9049 E-01 \\
8.1139 E-01 \\
8.2463 E-01 \\
8.7542 E-01 \\
9.4354 E-01 \\
9.4638 E-01 \\
9.9893 E-01 \\
1.0526 E+00 \\
1.1922 E+00 \\
1.1107 E+00 \\
1.1245 E+00 \\
1.1735 E+00 \\
1.1388 E+00 \\
1.4242 E+00 \\
1.2024 E+00 \\
9.3721 E-01 \\
1.2183 E+00 \\
1.5266 E+00 \\
6.0519 E-01 \\
1.3120 E+00 \\
1.2736 E+00 \\
7.8323 E-01 \\
5.8976 E-01 \\
1.8914 E+00 \\
4.8033 E+01\end{array}$ & $\begin{array}{l}1.6725 E+00 \\
1.3596 E+00 \\
1.1302 E+00 \\
1.1229 E+00 \\
1.0119 E+00 \\
8.8953 E-01 \\
8.6236 E-01 \\
8.5271 E-01 \\
8.3656 E-01 \\
8.2114 E-01 \\
8.0733 E-01 \\
7.9327 E-01 \\
7.7762 E-01 \\
7.6063 E-01 \\
7.4159 E-01 \\
7.2215 E-01 \\
7.0778 E-0 ! \\
6.9918 E-01 \\
6.9286 E-01 \\
6.8596 E-01 \\
6.9072 E-01 \\
6.9663 E-01 \\
7.0831 E-01 \\
7.2398 E-01 \\
7.3506 E-01 \\
7.6034 E-01 \\
7.8882 E-01 \\
8.0966 E-01 \\
8.2335 E-01 \\
8.7370 E-01 \\
9.4045 E-01 \\
9.4316 E-01 \\
9.9533 E-01 \\
1.0483 E+00 \\
1.1865 E+00 \\
1.1056 E+00 \\
1.1198 E+00 \\
1.1689 E+00 \\
1.1335 E+00 \\
1.4167 E+00 \\
1.1958 E+00 \\
9.3271 E-01 \\
1.2115 E+00 \\
1.5175 E+00 \\
6.0163 E-01 \\
1.3028 E+00 \\
1.2648 E+00 \\
7.7813 E-01 \\
5.8589 E-01 \\
1.8777 E+00 \\
4.7885 E+01\end{array}$ & $\begin{array}{l}1.6675 E+00 \\
1.3565 E+00 \\
1.1284 E+00 \\
1.1211 E+00 \\
1.0128 E+00 \\
8.9015 E-01 \\
8.6411 E-01 \\
8.5449 E-01 \\
8.3891 E-01 \\
8.2326 E-01 \\
8.0906 E-01 \\
7.9467 E-01 \\
7.7866 E-01 \\
7.6132 E-01 \\
7.4181 E-01 \\
7.2226 E-01 \\
7.0719 E-01 \\
6.9806 E-01 \\
6.9129 E-01 \\
6.8595 E-01 \\
6.9068 E-01 \\
6.9667 E-01 \\
7.0828 E-01 \\
7.2392 E-01 \\
7.3487 E-01 \\
7.6003 E-01 \\
7.8833 E-01 \\
8.0912 E-01 \\
8.2231 E-01 \\
8.7288 E-01 \\
9.4067 E-01 \\
9.4339 E-01 \\
9.9576 E-01 \\
1.0491 E+00 \\
1.1881 E+00 \\
1.1069 E+00 \\
1.1204 E+00 \\
1.1692 E+00 \\
1.1346 E+00 \\
1.4187 E+00 \\
1.1978 E+00 \\
9.3370 E-01 \\
1.2134 E+00 \\
1.5206 E+00 \\
6.0283 E-01 \\
1.3075 E+00 \\
1.2690 E+00 \\
7.8010 E-01 \\
5.8732 E-01 \\
1.8836 E+00 \\
4.7917 E+01\end{array}$ \\
\hline
\end{tabular}

证U.S. Government Printing Office: 1979 - 677-013/112 